\title{
Characterizing Aptamer-Based $\alpha$-Synuclein Fibril Inhibitors as a Potential Therapeutic Agent of Parkinson's Disease
}

\author{
by \\ Spencer Boisjoli \\ BSc (Hon), Carleton University 2018
}

A thesis submitted to the Faculty of Graduate and Postdoctoral Affairs in partial fulfillment of the requirements for the degree of

Master of Science

in

Chemistry

Carleton University

Ottawa, Ontario

C) 2020, Spencer Boisjoli 


\section{Abstract}

Parkinson's Disease (PD) is a neurodegenerative disorder pathologically characterized as loss of dopaminergic neurons and development of proteinaceous inclusions known as Lewy bodies (LBs). Predominant focus has been placed on the role of the protein $\alpha$-synuclein, given its high concentration within LBs and its increased tendency to form oligomeric and fibril species. Among the many treatment strategies that have been developed to circumvent PD neurodegeneration, this thesis investigates aptamers that were generated specifically for the detection of monomeric $\alpha$-synuclein and their fibril inhibition potential. Aptamers are single stranded oligonucleotide sequences designed for the recognition of target structures with high affinity and selectivity. Binding affinity and conformation analysis of five aptamer sequences, $\operatorname{ASYN}(1-5)$, for monomeric $\alpha$-synuclein were evaluated through a variety of methods. Electrochemical impedance spectroscopy, circular dichroism (CD), and microscale thermophoresis suggested ASYN(1-5) display high affinity for $\alpha$-synuclein, with ASYN2 being more selective. ASYN2 discriminated against similarly structured proteins, such as $\beta$ - and $\gamma$-synuclein, preferentially binding with monomeric $\alpha$-synuclein. ASYN2 truncations, exploiting potential binding domains within the aptamer, yielded six minimer sequences labelled A2m(1-6). Melting temperature and $\mathrm{CD}$ analysis suggested a centralized G-quadruplex binding motif was conserved within minimers A2m(3-5). Fibril inhibition assays determined whether ASYN(1-5) or A2m(1-6) were capable of hindering the aggregation of $\alpha$-synuclein into larger aggregate species. Both ASYN2 and A2m3 displayed inhibition potential at 1:1 molar ratios of aptamer to protein. Further investigation into ASYN2 and A2m3 could reveal its application as a PD therapeutic. 


\section{Acknowledgements}

I would like to express my sincere gratitude towards my thesis supervisor, Dr. Maria DeRosa, not only for her continuous support and leadership, but for allowing me the freedom and resources to pursue such a wonderful graduate education. My graduate studies enabled me to wholeheartedly live and learn chemistry, fueled through Maria's endless passion for the lovely world of aptamer chemistry.

I am grateful for the opportunity to pursue this research, made possible through the financial support of the Michael J. Fox Foundation (MJFF), Canadian Institutes of Health Research (CIHR), and Carleton University.

I am also grateful for my committee members, Dr. Matthew Holahan, Dr. Anatoli Ianoul, and Dr. Robert Crutchley, as well as the lovely chemistry department ladies, Caitlyn McKenzie and Chantelle Gravelle, for their advice with my thesis writing and support throughout my undergraduate and graduate years.

My special thanks towards Dr. Ranganathan Velu and Dr. Sathya Srinivasan for their never-ending guidance, generosity, and patience towards my daily barrage of questions. Their endless supply of humour and delicious Indian food had brightened my day on several occasions.

I would like to thank all my friends and colleagues within the Chemistry department for all the laughs and memories that were created during my time at Carleton University. Your assistance across various experiments, instrumentation, and in keeping morale high made the DeRosa lab an enjoyable and fulfilling experience.

Lastly, I would like to thank my family, my three essential kitties, and my amazing girlfriend for their unconditional love and support, and for keeping my head high and 
motivated at all times. You were a significant contributing factor to my overall success, whether you realized it or not, and I will forever be grateful for this wonderful period of my life. 


\section{Table of Contents}

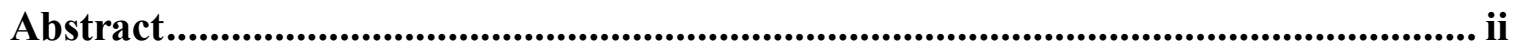

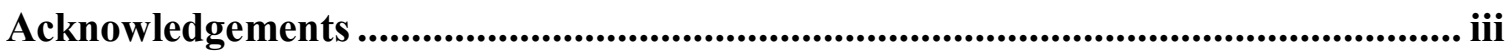

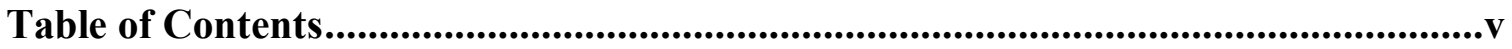

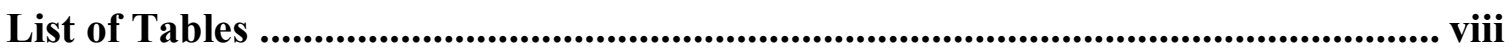

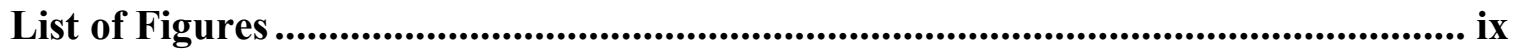

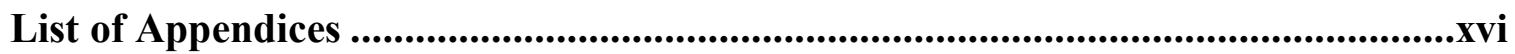

List of Abbreviations ..................................................................................................... xvii

Chapter 1: Introduction .................................................................................................1

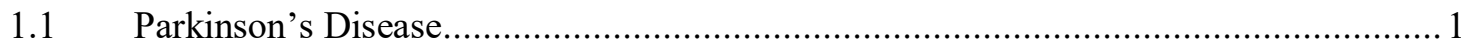

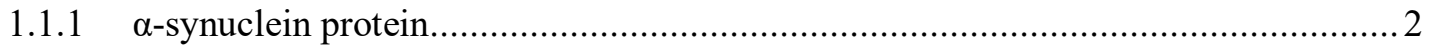

1.1.1.1 Predicted physiological role of $\alpha$-synuclein ................................................. 4

1.1.1.2 Aggregation mechanism of $\alpha$-synuclein ..................................................... 6

1.1.1.3 Neurotoxicity associated with $\alpha$-synuclein ............................................. 8

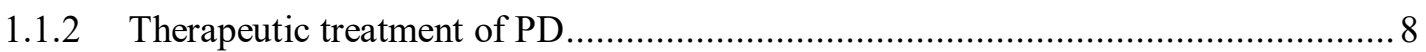

$1.2 \quad$ Aptamers

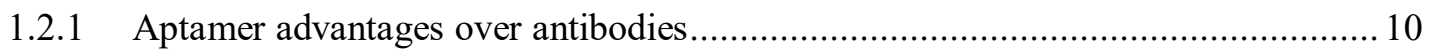

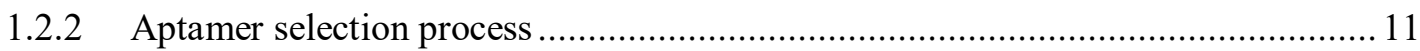

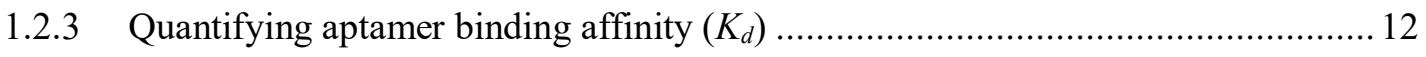

1.3 Aptamer characterization techniques and biosensor designs ................................... 14

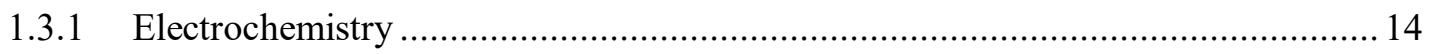

1.3.1.1 Aptamer immobilization on a solid surface ……........................................ 18

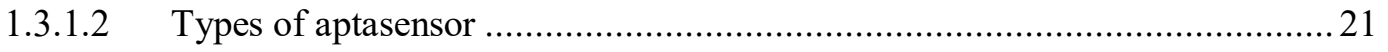

1.3.1.3 Electrochemical impedance spectroscopy (EIS) …….................................2. 


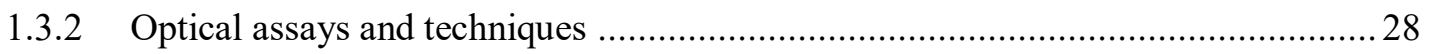

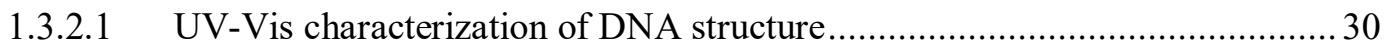

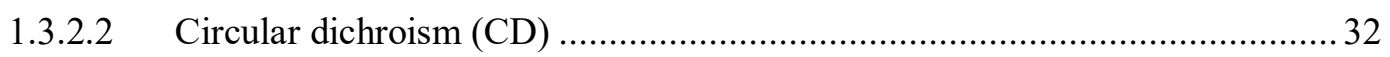

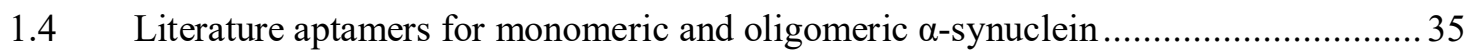

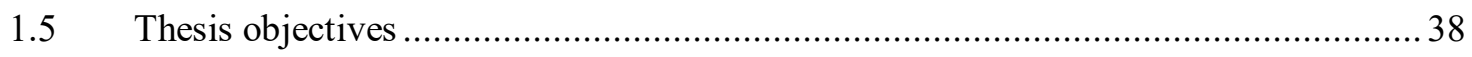

Chapter 2: Experimental.................................................................................39

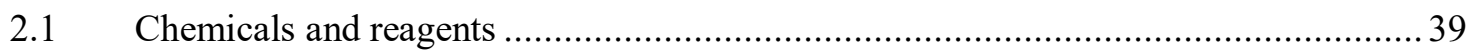

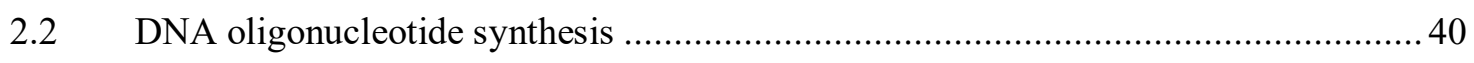

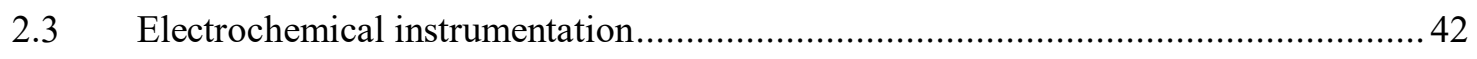

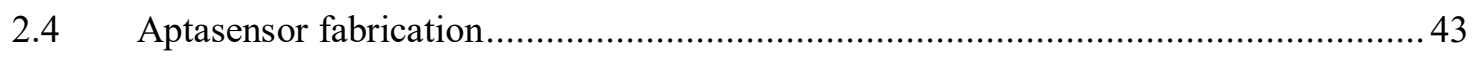

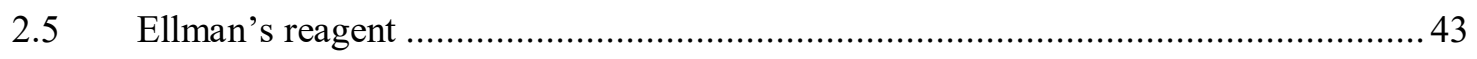

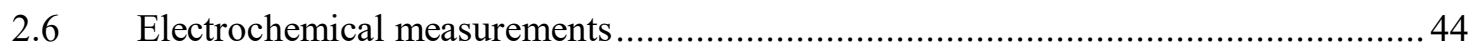

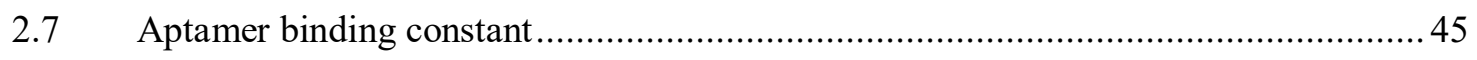

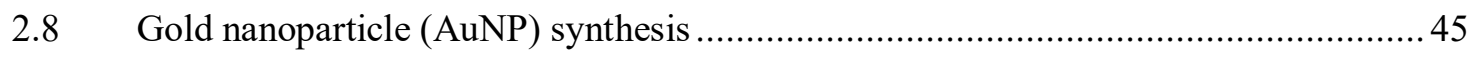

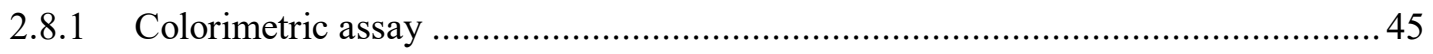

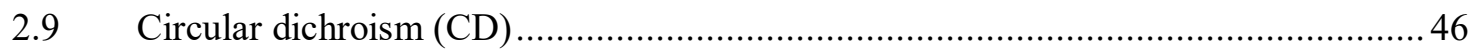

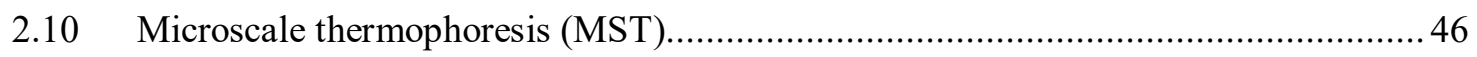

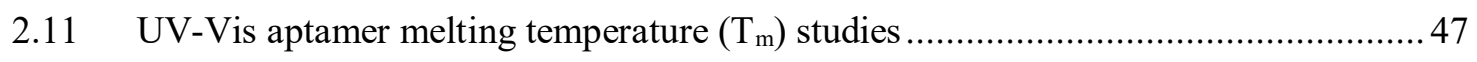

2.12 Preparation of $\alpha$-synuclein pre-formed fibrils (PFFs) ….................................... 47

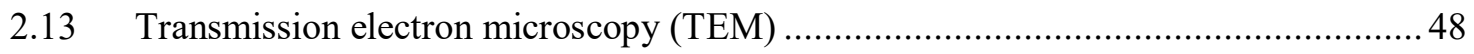

Chapter 3: Results and Discussion ................................................................................49

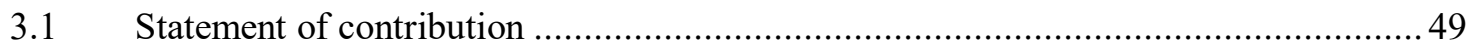

3.2 Development and characterization of the electrochemical aptasensor ......................50

3.2.1 Solid phase DNA synthesis by the phosphoramidite method ..............................50

3.2.2 Aptamer sequences and modifications ...................................................... 52

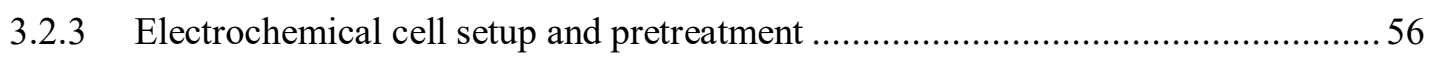




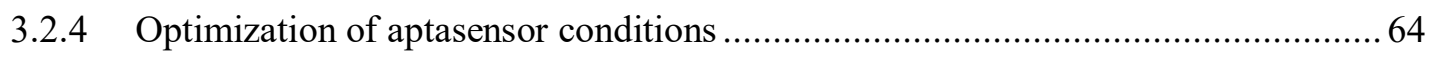

3.2.5 Monomeric $\alpha$-synuclein detection and specificity of the aptasensor ..................... 75

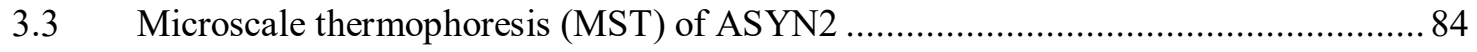

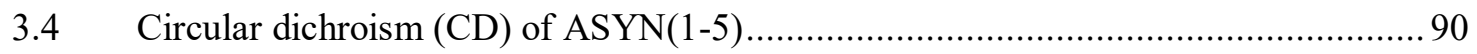

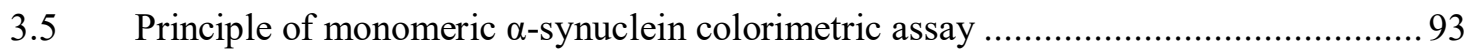

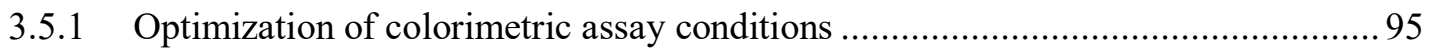

3.5.2 ASYN2 colorimetric assay for monomeric $\alpha$-synuclein ................................ 106

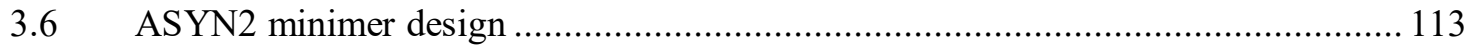

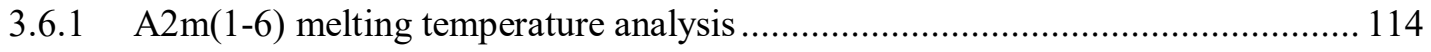

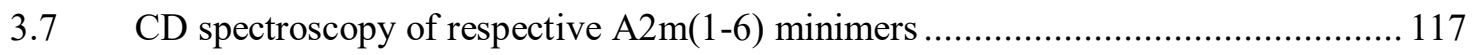

3.8 In vitro $\alpha$-synuclein fibril inhibition through the use of ASYN aptamers ................. 119

Chapter 4: Contributions to Knowledge and Future Direction ...............................128

Appendices ..............................................................................................................132

Appendix A Development of therapeutic strategies for ASYN2 drug delivery ..................... 132

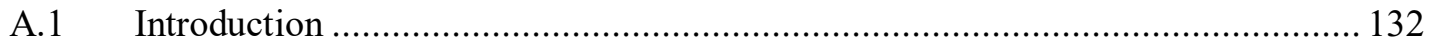

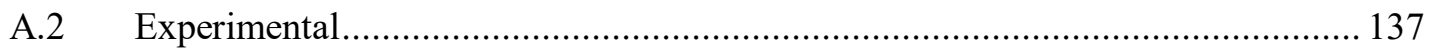

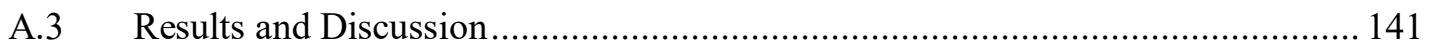

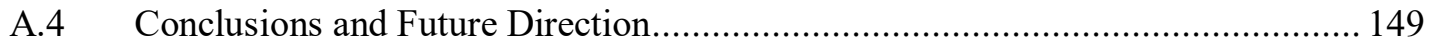

Appendix B Mass spectra of unmodified and C6-S-S modified ASYN(1-5) ...................... 150

References ..............................................................................................154 


\section{List of Tables}

Table 2. 1: Synthesized aptamer sequences used for characterization tests and inhibition assays. Colored segments denote the 5'-PBR (red), random region (black), 3'-PBR (blue), 5'-thiol modification (green), and 5'-Cy5 modification (orange). Extinction coefficients and theoretical $T_{m}$ were predicted suing IDT OligoAnalyzer software.............................................................................................41

Table 3. 1: $K_{d}$ values for selected aptamers against monomeric $\alpha$-synuclein and compared to currently existing $\alpha$-synuclein aptamers in literature. Aptamers selected for monomeric protein are denoted with (a) and those selected for the oligomeric protein are denoted with (b). ..............................................77

Table 5. 1: List of modified aptamer sequences involved in liposome synthesis where colored segments (Cy 5, orange) and (C6-S-S, green) denote the 5' modifications. ..................................................... 138 


\section{List of Figures}

Figure 1. 1: Protein structure of monomeric $\alpha$-synuclein comprised of three distinct domains. The $\mathbf{N}$ terminal domain (green, amino acids 1-60) consists of repeat KTKEGV regions responsible for its $\alpha$ helical conformation, the NAC domain (blue, amino acids 61-95) contains a hydrophobic center necessary for the formation of aggregate species, and C-terminal domain (purple, amino acids 96-140) retains a random coil conformation and is highly acidic at physiological pH. Familial PD mutations (red), and various post-translational modifications are also listed: ubiquitination (yellow), nitration (cyan), phosphorylation (orange). ${ }^{17}$

Figure 1. 2: Mechanism depicting various aggregation pathways of monomeric $\alpha$-synuclein and their various influences contributing to neurodegeneration within neuronal cells. Dimerization of monomeric $\alpha$-synuclein progresses into oligomeric species of unique conformations that have the potential to aggregate at a membrane interface or free in cytosolic media in larger fibril complexes. Misfolded $\alpha$-synuclein, oligomers, and protofibrils display seeding ability capable of propagating cell to cell, inducing the misfolding of endogenous $\alpha$-synuclein into larger fibril and LB structures. The prion-like characteristics of $\alpha$-synuclein is a large determinant into the neurotoxicity it inflicts on neuronal cells. ${ }^{24}$

Figure 1. 3: A $10 \mathrm{~mL}$ electrochemical cell consisting of an $\mathrm{Ag} \mid \mathrm{AgCl}$ reference electrode, platinum counter electrode, and a gold working electrode connected to a CHI660 potentiostat. CV and EIS measurements were performed in an electrolyte consisting of $5 \mathrm{mM}\left[\mathrm{Fe}(\mathrm{CN})^{6}\right]$.

Figure 1. 4: Non-specific binding of DNA bases (adenine, guanine, thymine, cytosine) to an exposed gold surface. ${ }^{91}$

Figure 1. 5: Electrochemical impedance spectroscopy (EIS) is represented through a Nyquist diagram. ${ }^{77}$.

Figure 1. 6: Illustration depicting the diffusion of electroactive ions in solution and the associated redox chemistry at the gold electrode-electrolyte interface. Ferrocyanide is oxidized to ferricyanide through Faradaic charge transfer that can be measured using EIS and CV techniques. A schematic of a double layer at the gold electrode surface in the presence of the $\mathrm{Fe}(\mathrm{CN}) 6^{3-4-}$ electroactive species. ${ }^{114}$ .27

Figure 1. 7: CD spectra differentiating $\alpha$-helix (solid line), $\beta$-sheet (long-dashed line), and random coiled (short-dashed line) protein secondary structures. ${ }^{137}$... .33

Figure 1. 8: CD spectra of parallel (A) vs anti-parallel (B) G-quadruplex motifs. The orientation of the guanine residues composing the $G$ tetrad, whether syn- or anti-, dictate the nature of the quadruplex and is easily distinguished through their CD spectra by the presence of inverse ellipticities. ${ }^{140}$

Figure 1. 9: SELEX process used to identify and generate $\operatorname{ASYN(1-5)}$ aptamer sequences for monomeric $\alpha$-synuclein. Reproduced with permission. ${ }^{147}$

Figure 3. 1: (A) Predicted secondary structures visualizing the free energy conformations of ASYN(15) sequences in accordance with RNAstructure. (B) The colored bases correspond to their respective 
base pairing probabilities while the free energy of the respective aptamers is determined in terms of kJ mol ${ }^{-1}$

Figure 3. 2: Representation of a G-tetrad composed of four guanine residues. A G-quadruplex motif is typically comprised of several tetrads where Hoogsteen bonding interactions are illustrated using a dashed line.

Figure 3. 3: (A) ASYN(1-5) sequence comparison with bases involved in G-quadruplex conformation highlighted in yellow with specific guanine tetrad units highlighted in red. (B) The respective G-score, determined using QGRS software, for ASYN(1-5) indicates that only ASYN(1-4) are capable of adopting the desired secondary structure. .55

Figure 3. 4: Cyclic voltammogram illustrating the cleaning process of the gold working electrode (AuE) in $1 \mathrm{M} \mathrm{H}_{2} \mathrm{SO}_{4}$ at various stages of electrochemical activation: the initial gold suface (curve a), the gold surface after 10 cycles (curve b), the gold surface after 40 cycles (curve c). Peak A corresponds to the double layer capacitance at the $\mathrm{AuE}-\mathrm{H}_{2} \mathrm{SO}_{4}$ interface, peaks $\mathrm{B}-\mathrm{E}$ correspond to the various redox reactions that are occurring at the gold interface within the oxide formation region of the $\mathrm{CV}$.........58

Figure 3. 5: (A) Schematic illustration of electrochemical aptasensor development and mechanism. Physical adsorption of thiolated ssDNA and 6-mercaptohexanol (MCH) occurred on a gold electrode surface in the presence of an electrolyte consisting of $5 \mathrm{mM}\left[\mathrm{Fe}(\mathrm{CN})_{6}\right]^{3-/ 4-}$ and $0.1 \mathrm{M} \mathrm{KCl} \mathrm{in} \mathrm{BFR-1.} \mathrm{B)}$ EIS spectra of the aptasensor at varying stages of the fabrication process lead to an observed increase in charge transfer resistance: (a) bare Au electrode, (b) Aptamer/Au electrode, (c) MCH/Aptamer/Au electrode, (d) Target/MCH/Aptamer/Au electrode. ${ }^{62}$ 61

Figure 3. 6: Representation of the Randles electrochemical equivalent circuit. $R_{s}$ represents the electrolyte resistance, $C_{d l}$ the double layer capacitance at the electrode-electrolyte interface, $R_{c t}$ the Faradaic charge transfer resistance between the redox probe and the aptasensor, $Z_{w}$ is $W_{\text {arburg }}$ coefficient representing the diffusion of charge from the gold interface to the bulk solution. 62

Figure 3. 7: Cyclic voltammogram depicting aptasensor fabrication in the presence of BFR-1 containing $5 \mathrm{mM}\left[\mathrm{Fe}(\mathrm{CN})_{6}\right]^{3-/ 4-}$ and $0.1 \mathrm{M} \mathrm{KCl}$. Voltammogram was performed over a potential range of $-0.5 \mathrm{~V}$ to $0.2 \mathrm{~V}$ at a scan rate of $200 \mathrm{mV}$. Respective aptasensor components are defined as: AuE/Buffer (curve a), Apt/AuE/Buffer (curve b), MCH/Apt/AuE/Buffer (curve c), Target/MCH/Apt/AuE/Buffer (curve d). . 64

Figure 3. 8: (A) Cyclic voltammogram depicting the available surface area of a gold electrode over a scan rate of $10 \mathrm{mV}$ to $200 \mathrm{mV}$, a potential range of $-0.1 \mathrm{~V}$ to $0.8 \mathrm{~V}$, and in the presence of an electrolyte consisting of $5 \mathrm{mM}\left[\mathrm{Fe}(\mathrm{CN})_{6}\right]^{3-/ 4-}$ and $0.1 \mathrm{M} \mathrm{KCl}$ in BFR-1. (B) calibration curve illustrating the gold surface area over varying scan rates $(10 \mathrm{mV}$ to $200 \mathrm{mV})$ where the Randles-Sevcik equation (Eq. 3.5) was applied to determine an average gold electrode surface area of $2.27 \mathbf{~ m m}^{2}(\mathrm{n}=4)$.

Figure 3. 9: Molecular structures of (A) TCEP and (B) DTT. 66

Figure 3. 10: Illustration of C6-S-S phosphoramidite modification at the 5'-terminal of ASYN(1-5). Cleavage in the presence of excess DTT or TCEP results in the formation of MCH and thiolated ssDNA 
in a 1:1 ratio, allowing for optimal coimmobilization of the gold surface during aptasensor optimization.

Figure 3. 11: Aptasensor optimization of DNA immobilization on a gold electrode surface. (A) DNA surface density measured over $1 \mu \mathrm{M}$ to $20 \mu \mathrm{M}$ resulted in a plateau at $10 \mu \mathrm{M}$. This concentration range was predicted to produce a high-density monolayer that could not effectively interact with the target of interest. As a result, $1 \mu \mathrm{M}$ DNA was used for the immobilization stage. (B) DNA incubation times were measured between $30 \mathrm{~min}$ and $1200 \mathrm{~min}$, where optimal DNA incubation time was determined to be 120 min.

Figure 3. 12: CD spectra of monomeric $\alpha$-synuclein. The characteristic presence of a negative peak at $190 \mathrm{~nm}$ indicates the protein retains a random coiled conformation when immersed in BFR-1, consistent with literature. 71

Figure 3. 13: Effect of BFR-1 containing varying $\mathrm{Mg}^{2+}$ concentrations ( $0 \mathrm{mM}$ to $\left.100 \mathrm{mM}\right)$ on secondary conformation of ASYN2. (A) $\mathrm{Mg}^{2+}$ in the absence of target influenced ASYN2 to become more structured as $\mathrm{Mg}^{2+}$ concentration increased to $10 \mathrm{mM}$. (B) $\mathrm{Mg}^{2+}$ in the presence of monomeric $\alpha$ synuclein influenced ASYN2 to adopt greater B-DNA characteristics, increase in $270 \mathrm{~nm}$ and decreased in $240 \mathrm{~nm}$ peaks respectively, as $\mathrm{Mg}^{2+}$ concentrations were increased to $100 \mathrm{mM}$. 72

Figure 3. 14: Predicted secondary structure of ASYN2R according to RNAstructure. ASYN2R bears a close resemblance to ASYN2

Figure 3. 15: Aptasensor optimization of MCH passivation on gold electrode surface. MCH concentrations between $1 \mathrm{mM}$ and $15 \mathrm{mM}$ provided no change in resistance, indicating that this concentration range is in excess and does not alter monolayer formation significantly with increasing concentration. $1 \mathrm{mM}$ MCH was determined to be optimal.

Figure 3. 16: Application of rinsing buffer (BFR-4) to remove non-specifically bound DNA and MCH from the gold electrode surface. It was determined that 15 min and 30 min washing steps are superimposed, indicating that 15 min was sufficient.

Figure 3. 17: Nyquist diagram of ASYN2 in the presence of monomeric $\alpha$-synuclein from $1 \mathrm{nM}$ to 1000 $\mathrm{nM}$ illustrated an increasing $\mathbf{R}_{\mathrm{ct}}$ with increasing target presence.

Figure 3. 18: EIS measurements illustrating the binding affinity of (a) ASYN1, (b) ASYN2, (c) ASYN2R, (d) ASYN3, (e) ASYN4, and (f) ASYN5 aptasensors in the presence of monomeric $\alpha$ synuclein. $K_{d}$ values were determined in SigmaPlot using nonlinear regression analysis. $\Delta R a t i o=\Delta S / \Delta P$ where $\Delta S=R_{c t(T a r g e t)}-R_{c t(A p t a s e n s o r)}$ and $\Delta P=R_{\text {ct(Aptasensor) }}-R_{\text {ct(Au electrode). }}$ The insets represent the linear dynamic range of the respective aptasensors over the selected concentration range.

Figure 3. 19: Selectivity of ASYN2 aptasensor in the presence of $1 \mu M$ of the respective targets. ASYN2 retained high affinity for monomeric forms of $\alpha$-synuclein while showing unexpectedly low affinity for the A53T mutant variant. 79 
Figure 3. 20: Protein sequence of A53T $\alpha$-synuclein. Molecular structure of alanine (Ala,A) and threonine (Thr,T) that underwent a missense point mutation at the $53^{\text {rd }}$ position. The substitution of a methyl for a hydroxyl appears to significantly alter binding interactions. 80

Figure 3. 21: TEM of A53T protein indicating the extensive aggregation that had occurred prior to electrochemical analysis.. 81

Figure 3. 22: Protein structure comparisons between $\alpha$-synuclein with $\beta$-synuclein and $\gamma$-synuclein respectively. Similarities across protein sequences have been highlighted in red and were performed using Clustal Omega.

Figure 3. 23: MST experimental setup. ${ }^{199}$ 85

Figure 3. 24: MST fluorescence timetrace depicting different phases of the experiment: the initial fluorescence of ASYN2-Cy5 (I), IR laser activation induces a temperature jump and decreases fluorescence (II), thermophoresis occurs as unbound and bound complexes migrate from the heated region until a steady state is achieved (III), deactivation of the IR laser causes a second temperature jump to occur (IV), diffusion of species into depleted region occurs to increase solution homology (V). The blue region denotes the cold period prior to laser activation and the red region denotes thermophoretic migration upon laser activation. As higher concentrations of ligand are titrated into the solution containing ASYN2-Cy5, the respective timetrace changes as a result. ASYN2 initially begins as an unbound aptamer and gradually plateaus in a bound aptamer-target complex. .86

Figure 3. 25: MST detection of ASYN2 $K_{d}$ in the presence of different synuclein structures: (A) monomeric $\alpha$-synuclein, (B) A53T mutant $\alpha$-synuclein, (C) $\beta$-synuclein, (D) $\gamma$-synuclein. The normalized fluorescence $\left(\Delta F_{n o r m}\right)$ is determined through the ratio of $F_{\text {hot }} / F_{\text {cold }}$ where $F_{\text {hot }}$ is the fluorescence within the red area and $F_{\text {cold }}$ is the fluorescence within the blue area. .88

Figure 3. 26: CD spectra of ASYN(1-5) sequences at a concentration of $18.75 \mu \mathrm{M}$ in BFR-1. .090

Figure 3. 27: Illustration of respective (A) anti- and (B) syn-guanine orientations within a G-tetrad. The ratio of syn-/anti-guanine within an aptamer dictates the parallel or antiparallel nature of the corresponding G-quadruplex. ${ }^{207}$ .91

Figure 3. 28: CD spectra of ASYN2 in BFR-1 illustrating the conformational changes within the aptamer sequence as the concentration of monomeric $\alpha$-synuclein is increased from $(0.19 \mu \mathrm{M}-1.875$ $\mu M)$. The arrows indicate the directional change of the ASYN2 peaks.

Figure 3. 29: A calibration curve illustrating the CD conformational changes at $216 \mathrm{~nm}$ between ASYN2 and monomeric $\alpha$-synuclein. Sigmaplot was used to determine a $K_{d}$ value of approximately 5 $\mu M$ for ASYN2. 93

Figure 3. 30: TEM image of synthesized citrate capped AuNPs. The AuNPs appeared as a wine-red solution with an average diameter of $15 \mathrm{~nm}$ .94 
Figure 3. 31: AuNP colorimetric mechanism depicting optical biosensor fabrication and monomeric $\alpha$ synuclein detection. Apt: aptamer; NaCl: sodium chloride; AuNP: gold nanoparticle, $\alpha$-syn: monomeric $\alpha$-synuclein.

Figure 3. 32: (A) Optimization of AuNP concentration (0.75 nM - $7.53 \mathrm{nM}$ ) for colorimetric assays. (B) UV-Vis spectra of the corresponding AuNP concentrations where AuNP concentrations of $3.77 \mathrm{nM}$ were applied for further colorimetric assay analysis. .96

Figure 3. 33: (A) Optimization of $\mathrm{NaCl}$ stock concentration $(0.25 \mathrm{M}-0.50 \mathrm{M})$ for colorimetric assays in the presence of $0.75 \mathrm{nM}-7.53 \mathrm{nM}$ AuNPs. (B) UV-Vis spectra of respective NaCl stock concentrations $(0.25 \mathrm{M}-0.50 \mathrm{M})$ in the presence of $3.77 \mathrm{nM}$ AuNPs. (C) Plot of the absorbance ratio (A625/A525) vs $\mathrm{NaCl}$ stock concentration where a plateau is observed at $0.4 \mathrm{M}$. In each $\mathrm{NaCl}$ measurement $30 \mu \mathrm{L}$ of the respective $\mathrm{NaCl}$ stock were added to the AuNP solution. .97

Figure 3. 34: $\mathrm{NaCl}$ concentration range $(0 \mathrm{mM}-80 \mathrm{mM})$ in the presence of $3.77 \mathrm{nM}$ AuNPs. A relatively linear trend was observed, and $60 \mathrm{mM}$ was deemed optimal for the colorimetric assays. .98

Figure 3. 35: Non-specific DNA interaction with $3.77 \mathrm{nM}$ AuNPs and $60 \mathrm{mM}$ NaCl. (A) UV-Vis spectra of DNA $(0.00 \mu \mathrm{M}-0.35 \mu \mathrm{M})$. (B) Calibration curve displaying the absorbance ratio $\left(\mathrm{A}_{625} / \mathrm{A}_{525}\right)$ vs DNA $(0.00 \mu \mathrm{M}-0.35 \mu \mathrm{M})$ where a plateau occurred at $0.25 \mu \mathrm{M}$. (C) Visual colour change of the AuNPs coated in various concentrations of DNA $(0.00 \mu \mathrm{M}-0.35 \mu \mathrm{M})$. .99

Figure 3. 36: (A) Monomeric $\alpha$-synuclein ( $0 \mathrm{nM}$ - $1000 \mathrm{nM})$ in presence of $3.77 \mathrm{nM}$ AuNPs. Over the selected concentration range, no AuNP aggregation was observed through AuNP-target interactions. (B) Optical colorimetric biosensor in the presence of monomeric $\alpha$-synuclein $(0 \mathrm{nM}-1000 \mathrm{nM})$. It is apparent that $\alpha$-synuclein concentrations exceeding $200 \mathrm{nM}$ protect AuNPs from salt-induced aggregation. 100

Figure 3. 37: Schematic representation of proposed PDDA colorimetric detection assay. Apt: aptamer; PDDA: poly(diallyldimethylammonium chloride); AuNP: gold nanoparticle; $\alpha$-syn: monomeric $\alpha$ synuclein. 101

Figure 3. 38: Optimization of PDDA on $3.77 \mathrm{nM}$ AuNPs. A plateau was observed at $1.89 \times 10^{-4} \mathrm{mM}$, proving to be an ideal PDDA concentration for the complete aggregation of AuNPs. 103

Figure 3. 39: (A) The optimization of ASYN2 was performed using $3.77 \mathrm{nM}$ AuNPs and $1.89 \times 10^{-4} \mathrm{mM}$ PDDA. ASYN2 $(0.00 \mu \mathrm{M}-0.45 \mu \mathrm{M})$ The inset depicts a smaller concentration range $(0.00 \mu \mathrm{M}-0.10$ $\mu M)$. (B) UV-Vis spectra of ASYN2 $(0.00 \mu \mathrm{M}-0.10 \mu \mathrm{M})$. (C) Calibration curve of absorbance $\left(\mathrm{A}_{680} / \mathrm{A}_{525}\right)$ depicts a plateau at $0.07 \mu \mathrm{M}$ ASYN2. 104

Figure 3. 40: CD spectra of ASYN2 in the presence of monomeric $\alpha$-synuclein (1:1 molar ratio) and PDDA $\left(1.89 \times 10^{-4} \mathrm{mM}\right)$. It was determined that PDDA does not induce conformational changes upon hybridization with ASYN2. 105

Figure 3. 41: Colorimetric assay using $3.77 \mathrm{nM}$ AuNPs, $70 \mathrm{nM}$ ASYN2, $1.89 \times 10^{-4} \mathrm{mM}$ PDDA, monomeric $\alpha$-synuclein $(0 \mathrm{nM}-250 \mathrm{nM}$ ). (A) Addition of AuNP/Apt/PDDA/ $\alpha$-syn turned the solution purple across the target concentration range. (B) Addition of AuNP/Apt/a-syn/PDDA kept the solution 
red across the target concentration range. It was observed in both cases that a darker colour was obtained at lower concentrations, further exhibiting the protective effects of $\alpha$-synuclein on AuNPs at higher concentrations.

Figure 3. 42: TEM images of AuNP solutions in the presence of varying optimized parameters. (A) 3.77 nM AuNP blank, (B) AuNPs + $70 \mathrm{nM}$ ASYN2, (C) AuNPs $+1.89 \times 10^{-4} \mathrm{mM}$ PDDA, (D) AuNPs + ASYN2 + PDDA, (E) AuNPs + ASYN2 + PDDA + 250 nM $\alpha$-syn, (F) AuNPs + ASYN2 + PDDA + 2500 nM $\alpha$-syn. 108

Figure 3. 43 Proposed interaction between monomeric $\alpha$-synuclein acidic C-terminal domain and the AuNP surface through citrate displacment. 109

Figure 3. 44: Predicted secondary structure of ASYN2 according to RNAstructure software with labelled minimer sequences: 5'-stem loop structure (A2m1), 3'-stem loop structure (A2m2), no 3'-PBR (A2m3), no 5'-PBR (A2m4), no 5'- or 3'-PBRs (A2m5), centralized G-quadruplex binding motif (A2m6).

Figure 3. 45: Minimer melting temperature $\left(T_{m}\right)$ analysis at $295 \mathrm{~nm}$ over a temperature range of $20^{\circ} \mathrm{C}$ $-90^{\circ} \mathrm{C}$ : (A) A2m1, (B) A2m2, (C) A2m3, (D) A2m4, (E) A2m5, (F) A2m6. Hypochromicity is observed in $\mathrm{C}-\mathrm{F}$ indicated the denaturing of the centralized G-quadruplex motif. A2m6 displays two curves: increasing temperature ramp (blue curve) and decreasing temperature ramp (orange curve). The nonsuperimposable curves indicate hysteresis among the two curves and reflects the potential instability of A2m6. 116

Figure 3. 46: CD spectra of A2m(1-6) sequences at a concentration of $18.75 \mu \mathrm{M}$ in BFR-1.

Figure 3. 47: TEM images illustrating the uranyl acetate (UA) stain used to visualize the $\alpha$-synuclein fibril species (A), monomeric $\alpha$-synuclein following a seven-day incubation (B), random transferrin receptor aptamer (TRA) following a seven-day incubation in the presence of a PFF $\alpha$-synuclein seed protein. The seven-day incubation period was kept at $37^{\circ} \mathrm{C}$ and constant agitation. Scale bar, $1 \mu \mathrm{m}$.

Figure 3. 48: TEM images comparing the inhibitive effects of ASYN(1-5) and M5-15 on monomeric $\alpha$ synuclein fibril formation. The respective aptamers were incubated for seven days in the presence of an $\alpha$-synuclein PFF at $37^{\circ} \mathrm{C}$ and with constant agitation. (A) $\alpha$-synuclein fibrils (PFF) in the absence of aptamer, (B) ASYN2, (C) M5-15, (D) ASYN1, (E) ASYN3, (F) ASYN4, (G) ASYN5. All images were obtained in aptamer:protein molar ratios of 10:1. Scale bar, $1 \mu \mathrm{m}$. 122

Figure 3. 49: TEM images comparing the aggregation inhibition of ASYN2 with respect to literature aptamers selected for monomeric and oligomeric $\alpha$-synuclein: (A) F5R1, (B) F5R2, (C) ASYN2, (D) TSO530, (E) T-SO508. All images were obtained in aptamer:protein molar ratios of 10:1 following a seven-day incubation period at $37^{\circ} \mathrm{C}$ and constant agitation. Scale bar, $1 \mu \mathrm{m}$. 123

Figure 3. 50: TEM images of the inhibition potential of ASYN2 in molar ratios of ASYN2: $\alpha$-synuclein PFF of: (A) 10:1, (B) 1:1, (C) 0.5:1, (D) 0.1:1. All images were obtained following a seven-day incubation period at $37^{\circ} \mathrm{C}$ and constant agitation. Scale bar, $1 \mu \mathrm{m}$. 124 
Figure 3. 51: TEM images comparing the inhibitive effects of $A 2 m(1-6)$ on monomeric $\alpha$-synuclein fibril formation. The respective aptamers were incubated for seven days in the presence of an $\alpha$ synuclein PFF at $37^{\circ} \mathrm{C}$ and with constant agitation. (A) A2m1, (B) A2m2, (C) A2m3, (D) A2m4, (E) A2m5, (F) A2m6. All images were obtained in aptamer:protein molar ratios of 1:1. Scale bar, $1 \mu \mathrm{m}$.

Figure 5. 1: Literature DNA aptamers exhibiting specific binding for transferrin receptors along endothelial cells. A) Full-length transferrin receptor aptamer (TRA); B) truncated TfR minimer exploiting the 5'-stem loop within the full-length sequence; C) therapeutic bifunctional aptamer utilizing a chimera between TfR and specific drug delivery sequence.

Figure 5. 2: Lipids involved in liposome synthesis: A) POPC; B) DDAB; C) DSPE-PEG(2000); D) DSPE-PEG(2000) maleimide

Figure 5. 3: Quantification of encapsulated ASYN2 (20x dilution, blue curve) and tethered exterior TRA (200x dilution, orange curve) following liposome synthesis. 144

Figure 5. 4: Stewart assay results used to determine the final lipid composition of the previously synthesized liposomes. A calibration curve was produced over a lipid concentration of $0-0.025 \mathrm{mg} / \mathrm{mL}$ at a constant concentration of ferrothiocyanate. The orange dot in the right figure corresponds to the lipid concentration of the synthesized liposomes, determined to have a final concentration of $25.1 \pm 0.61$ $\mathrm{mg} / \mathrm{mL}$ (70\% yield). 146

Figure 5. 5: Theoretical chimera aptamers: A) ASYN2-TRA; B) ASYN2-TfR; C) A2m3-TRA; D) A2m3-TfR. 147

Figure 5. 6: Deconvoluted ESI-MS spectra of ASYN1. 150

Figure 5. 7: Deconvoluted ESI-MS spectra of ASYN2. 150

Figure 5. 8: Deconvoluted ESI-MS spectra of ASYN3. 150

Figure 5. 9: Deconvoluted ESI-MS spectra of ASYN4. 151

Figure 5. 10: Deconvoluted ESI-MS spectra of ASYN5. 151

Figure 5. 11: Deconvoluted ESI-MS spectra of A2TMR. 151

Figure 5. 12: Deconvoluted ESI-MS spectra of ASYN2-TM. 152

Figure 5. 13: Deconvoluted ESI-MS spectra of ASYN3-TM. 152

Figure 5. 14: Deconvoluted ESI-MS spectra of ASYN4-TM. 152

Figure 5. 15: Deconvoluted ESI-MS spectra of ASYN5-TM. 153 


\section{List of Appendices}

Appendix A Development of therapeutic strategies for ASYN2 drug delivery........ 126

A.1 Introduction................................................... 126

A.2 Experimental...............................................131

A.3 Results and Discussion......................................... 135

A.4 Conclusion...................................................143

Appendix B Mass spectra of unmodified and modified C6-S-S ASYN(1-5).......... 144 


\section{List of Abbreviations}

$\alpha$-synuclein:

$\mathrm{A} \beta$ :

A:

$\mathrm{AD}$ :

AgNP:

AuE:

AuNP:

$\mathrm{C}$ :

CD:

$\mathrm{C}_{\mathrm{dl}}$ :

$\mathrm{CE}$ :

CPG:

CSF:

CV:

DA:

$\mathrm{DiH}_{2} \mathrm{O}$ :

DLB:

DJ-1:

DMT:

DNA:

dsDNA:

DTT:

EIS:

G:

HOMO:

IHP:

ITC:

$\mathrm{K}_{\mathrm{d}}$ :

LB:

L-DOPA:

LDR:

LN:

LOD:

LRRK2:

LSPR:

LUMO:

MAB:

MB:

$\mathrm{MCH}$ :
Alpha-synuclein

Amyloid beta

Adenine

Alzheimer's Disease

Silver nanoparticle

Gold working electrode

Gold nanoparticle

Cytosine

Circular dichroism

Capacitor double layer

Counter electrode

Controlled pore glass

Cerebrospinal fluid

Cyclic voltammetry

Dopamine

Deionized water

Dementia with Lewy Bodies

Parkinson's Disease protein 7

4,4'-dimethoxytrityl

Deoxyribonucleic acid

Double stranded DNA

Dithiothreitol

Electrochemical impedance spectroscopy

Guanine

Highest occupied molecular orbital

Inner Helmholtz plane

Isothermal titration calorimetry

Dissociation equilibrium constant

Lewy Body

Levodopa

Linear dynamic range

Lewy Neurite

Limit of detection

Leucine-rich repeat kinase 2

Localized surface plasmon resonance

Lowest unoccupied molecular orbital

Molecular beacon aptamer

Methylene blue

6-mercaptohexanol 


$\begin{array}{ll}\text { MSA: } & \text { Multiple Systems Atrophy } \\ \text { MST: } & \text { Microscale thermophoresis } \\ \text { NAC: } & \text { Non-amyloid-beta component } \\ \text { OHP: } & \text { Outer Helmholtz plane } \\ \text { PAGE: } & \text { Polyacrylamide gel electrophoresis } \\ \text { PBR: } & \text { Primer binding region } \\ \text { PBS: } & \text { Phosphate buffered saline } \\ \text { PD: } & \text { Parkinson's Disease } \\ \text { PDDA: } & \text { Poly(diallyldimethyl ammonium chloride) } \\ \text { PFF: } & \text { Pre-formed fibril } \\ \text { PINK1: } & \text { PTEN-induced kinase I } \\ \text { PRKN: } & \text { Parkin gene } \\ \text { QD: } & \text { Quantum dot } \\ \text { Rct: } & \text { Charge-transfer resistance } \\ \text { RE: } & \text { Reference electrode } \\ \text { Redox: } & \text { Reduction-oxidation } \\ \text { RNA: } & \text { Ribonucleic acid } \\ \text { R: } & \text { Solution resistance } \\ \text { SAM: } & \text { Self-assembled monolayer } \\ \text { SCE: } & \text { Saturated calomel electrode } \\ \text { SELEX: } & \text { Systematic evolution of ligands by exponential enrichment } \\ \text { SHE: } & \text { Standard hydrogen electrode } \\ \text { SNARE: } & \text { Soluble N-ethylmaleimide-sensitive factor attachment protein } \\ & \\ \text { SNCA: } & \text { Synuclein alpha } \\ \text { SPR: } & \text { Surface plasmon resonance } \\ \text { ssDNA: } & \text { Single stranded DNA } \\ \text { T: } & \text { Thymine } \\ \text { TCEP: } & \text { Tris(2-carboxyethyl)phosphine } \\ \text { TDS: } & \text { Thermal difference spectra } \\ \text { TEM: } & \text { Transmission electron microscopy } \\ \text { TR: } & \text { Transferrin receptor } \\ \text { TfR: } & \text { Truncated transferrin receptor aptamer } \\ T_{\mathrm{m}}: & \text { Melting temperature } \\ \text { TRA: } & \text { Transferrin receptor aptamer } \\ \text { UA: } & \text { Uranyl acetate } \\ \text { UV-Vis: } & \text { Working electrode } \\ \text { WE: } & \\ Z_{\mathrm{w}}: & \end{array}$




\section{Chapter 1: Introduction}

\subsection{Parkinson's Disease}

Parkinson's Disease (PD) is a prevalent neurodegenerative disorder that predominantly affects $1-2 \%$ of those aged 60 years or older with men being at higher risk. ${ }^{1}$ Initially characterized by James Parkinson in 1817, PD is clinically described through its motor characteristics comprising bradykinesia or slowness of movement, resting tremors, postural instability, and muscular rigidity. ${ }^{2,3}$ These four symptoms, along with freezing and flexed posture, are the cardinal features of parkinsonism. ${ }^{4}$ Non-motor neurological symptoms also arise such as depression, anxiety, and fatigue due to subsequent neurodegeneration of adjacent regions of the brain, such as the basal ganglia. ${ }^{4}$ The pathological motor symptoms of PD result from the progressive degeneration of dopaminergic neurons within the substantia nigra of the midbrain, and the formation of $\alpha$ synuclein-rich proteinaceous inclusions known as Lewy Bodies (LBs) and Lewy Neurites (LNs) that persist within surviving neurons. ${ }^{1}$ LBs are not strictly limited to PD as LBs have been discovered in similar neurodegenerative disorders such as Alzheimer's Disease (AD) ${ }^{5}$ Dementia with Lewy Bodies (DLB), ${ }^{1,6}$ and multiple systems atrophy (MSA). ${ }^{1,2,7}$ LBs were initially presumed to be responsible for the neurodegenerative properties associated with PD but were later suggested as a protective mechanism to minimize neurodegenerative effects. ${ }^{8,9}$

PD has been found to be both idiopathic/sporadic based, or familial in nature. ${ }^{1,2,4}$ Idiopathic PD encompasses the majority of PD cases, approximately $90 \%$, and is largely associated with unknown environmental factors. ${ }^{1,2}$ Studies have suggested that those living in rural areas with exposure to pesticides such as paraquat, maneb, and rotenone can 
develop neurodegeneration through mitochondrial complex I inhibition and oxidative stress. Certain industrial occupations such as welding, mining, and cleaning with increased exposure to iron and manganese metals alters uptake of these metal ions and can lead to their accumulation and corresponding neurodegeneration. ${ }^{2}$ Familial cases of PD have led to growing interest as additional gene mutations are discovered and researched. Several autosomal-dominant and -recessive genes have been identified as a cause of familial PD where $\alpha$-synuclein $(S N C A)$, leucine-rich repeat kinase 2 (LRRK2), parkin (PRKN), PTENinduced Kinase I (PINK1), and DJ-1 are the most common. ${ }^{2}$ The SNCA gene contains several point mutations, A30P, E46K, and A53T, that lead to an overexpression in $\alpha$ synuclein through its duplication and triplication..$^{2,7,10,11}$ The A53T missense mutation, resulting from the change of an alanine to threonine residue at position 53 within the $\alpha$ synuclein protein, has been hypothesized to lead to a higher tendency for aggregate

formation and increased neurotoxicity from its overexpressed cellular concentration. ${ }^{7,10}$ For these reasons, $\alpha$-synuclein has been a biomarker and target of interest when developing inhibition strategies for the treatment of PD.

\subsection{1 $\quad \alpha$-synuclein protein}

$\alpha$-synuclein is a $14.4 \mathrm{kDa}$ protein consisting of 140 amino acid residues, encoded by the $S N C A$ gene, where $\alpha$-synuclein pathology is believed to be a hallmark of PD. ${ }^{1,10}$ Under physiological conditions $\alpha$-synuclein is a soluble, intrinsically disordered protein with an uncoiled conformational nature and absence of a hydrophobic pocket. ${ }^{12} \alpha$ synuclein primarily exists as a monomeric protein ${ }^{13,14}$ with some studies suggesting its 
existence as a tetrameric species. ${ }^{15,16}$ The protein structure is comprised of three principle domains and is illustrated in Figure 1.1.

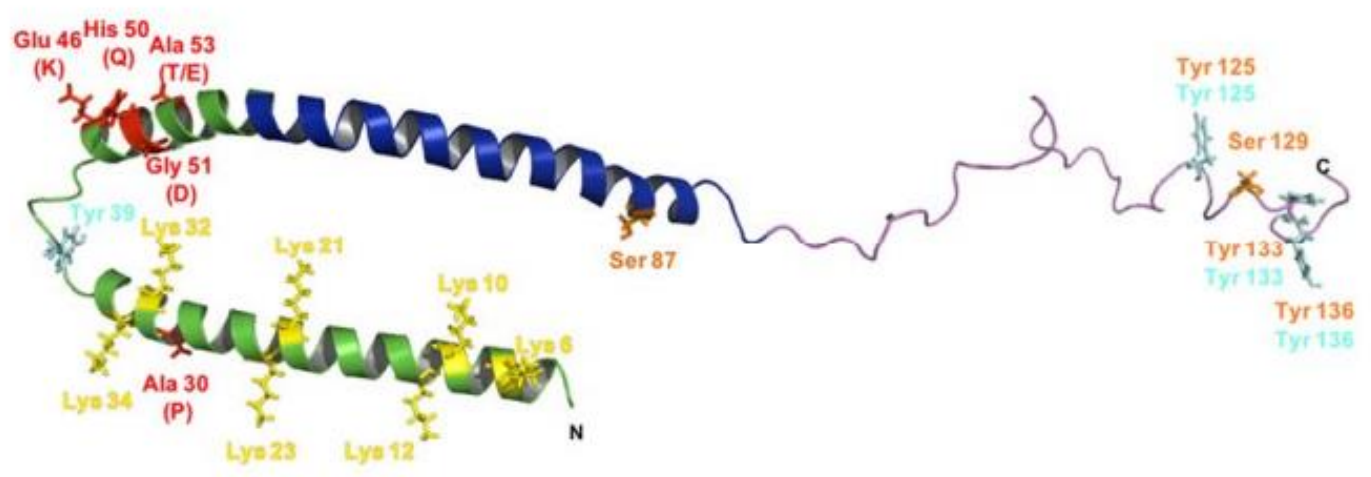

Figure 1. 1: Protein structure of monomeric $\alpha$-synuclein comprised of three distinct domains. The Nterminal domain (green, amino acids 1-60) consists of repeat KTKEGV regions responsible for its $\alpha$ helical conformation, the NAC domain (blue, amino acids 61-95) contains a hydrophobic center necessary for the formation of aggregate species, and C-terminal domain (purple, amino acids 96-140) retains a random coil conformation and is highly acidic at physiological pH. Familial PD mutations (red), and various post-translational modifications are also listed: ubiquitination (yellow), nitration (cyan), phosphorylation (orange). Reproduced with permission from De Gruyter. ${ }^{17}$

The N-terminal domain stretches amino acids 1-60 containing conserved amino acid repeats with the general motif KTKEGV. ${ }^{10,11}$ These conserved segments are capable of adopting an $\alpha$-helical conformation, allowing its terminal region to interact with lipid membranes. ${ }^{1,10}$ The central non- $\mathrm{A} \beta$ component (NAC) domain (amino acids 61-95) is amyloidogenic, having been compared with amyloid peptides in $\mathrm{AD}$ due to their similar aggregation tendency and soluble characteristics. ${ }^{1,18}$ The C-terminal domain (amino acids 96-140) has an unstructured, random coil nature that is highly acidic at physiological $\mathrm{pH}$ (-9) due to the abundance of glutamate residues within the tail region. ${ }^{1,10}$ It is believed that the C-terminal domain contains active sites responsible for interactions with other proteins or small molecules. ${ }^{19}$ Long range interactions between the extremely negative C-terminal domain and the slightly positive NAC region confer structural properties to the disordered 
protein that can have a substantial impact on its neurodegenerative and neuroprotective characteristics. ${ }^{3,20}$

$\alpha$-synuclein resides within the synuclein protein family consisting of $\beta$-synuclein and $\gamma$-synuclein; $\beta$ - and $\gamma$-contain sequence similarities to $\alpha$-synuclein without the inherent ability to aggregate and form higher ordered oligomeric or fibril species. ${ }^{1,11,21}$ All three synuclein proteins are located within presynaptic terminals, but the main difference among these protein structures is a lack of the NAC region believed to be responsible for the fibril formation observed in PD. ${ }^{3}$ In healthy individuals, $\alpha$-synuclein resides within the terminal ends of neuronal cells and composes approximately $1 \%$ of total cytosolic protein. ${ }^{7}$ The prevalence of presynaptic $\alpha$-synuclein is due to its preference for high curvature membranes, such as synaptic vesicles due to their small radius, and acidic lipids, which maintain high cytoplasmic concentrations within synaptic vesicular membrane. ${ }^{1}$

\subsubsection{Predicted physiological role of $\alpha$-synuclein}

The physiological function of $\alpha$-synuclein remains unknown, but several studies suggest a role in synaptic vesicle regulation and neurotransmitter release. ${ }^{7,22-24} \alpha$ synuclein's involvement with neurotransmitter release has been widely discussed through its interactions with the SNARE complex (Soluble NSF Attachment Protein Receptor), responsible for the fusion of vesicles with a lipid membrane. ${ }^{25}$ The SNARE complex overcomes the repulsive synaptic vesicle and the target membranes, overcoming the required energy barrier and bringing the vesicle into close proximity with the target membrane for fusion to occur. The SNARE complex incorporates itself into both the vesicle membrane (v-SNARE) and the target membrane (t-SNARE) where the 
simultaneous formation of $\mathrm{v}$ - and t-SNAREs cause the vesicle to transfer across the membrane bilayer. ${ }^{25}$ Knockout mice, those genetically engineered to not express specific proteins, are commonly used for $\alpha$-synuclein functional analysis. Triple knockout mice, lacking $\alpha-, \beta-, \gamma$-synuclein, were found to experience loss of neuronal function and synaptic plasticity ${ }^{26}$ while $S N C A$ knockout mice, only lacking $\alpha$-synuclein, were determined to decrease the recycling of synaptic vesicles and replenishment of reserve pools. ${ }^{24,27}$ One interesting study found that despite $\alpha-, \beta$-, and $\gamma$-synuclein not being directly responsible for neurotransmitter release, they play a critical role in nerve maintenance and function in the long term. ${ }^{28}$

The ability for $\alpha$-synuclein to exist in multiple conformations such as monomers, tetramers, oligomers, fibrils, LNs, and LBs influence neurodegenerative effects within neuronal cells as it interacts with numerous membranes and protein structures. ${ }^{1,10}$ Misfolding within $\alpha$-synuclein predominantly occurs through post-translational modifications (PTMS) such as phosphorylation, nitration, oxidation, and ubiquitination. PTMs have been found to alter physiological $\alpha$-synuclein secondary and tertiary structure, but also induce protein functionality leading to increased neurodegeneration and aggregation or enhanced neuroprotective effects. ${ }^{29}$ One of the most studied PTMs is phosphorylation at Ser-129 (PS-129) where it was determined that LBs contain a high degree of $\alpha$-synuclein exhibiting these PTMs, expressing concentrations over $90 \%{ }^{30,31}$ Studies indicate that PS-129 enhances aggregate formation and reduces degradation ${ }^{32}$ while its absence increases motor functionality and minimizes aggregate species formation. ${ }^{33}$ 


\subsubsection{Aggregation mechanism of $\alpha$-synuclein}

Figure 1.2 illustrates the multiple aggregation mechanisms of $\alpha$-synuclein through which neurotoxicity and aggregate concentration occur. ${ }^{24}$ Oligomers contain multiple misfolded $\alpha$-synuclein subunits with a propensity to aggregate into larger species but have not yet manifested fibril nature leading to LB formation.Studies have found that misfolded $\alpha$-synuclein has seeding attributes that can influence the rate at which these higher molecular weight aggregate species are developed. ${ }^{34-37}$ Oligomeric species composed of misfolded $\alpha$-synuclein display varying degrees of seeding potential with larger species exhibiting increased stability and susceptibility for seeding aggregation. ${ }^{38}$ Fibril and LB $\alpha$ synuclein morphology have been well-documented as fibril formation is energetically favorable following the nucleated polymerization of monomeric $\alpha$-synuclein. ${ }^{3}$ There is conflicting evidence about which $\alpha$-synuclein species contributes most significantly to PD toxicity as oligomers ${ }^{1,10}$ and fibrils ${ }^{39}$ increase toxicity exhibited throughout neuronal cells, while other studies hint at soluble monomeric forms of $\alpha$-synuclein and those exhibiting PTMs being the most detrimental. ${ }^{40}$

Several studies hypothesize the existence of $\alpha$-synuclein cell-to-cell transfer describing it as a prion-like mechanism of transmission of misfolded $\alpha$-synuclein protein to an adjacent neighboring cell. ${ }^{1,3,7,11,22,24,41}$ Prions are infectious proteins capable of inducing misfolding in native proteins, $\alpha$-synuclein for example, where misfolded proteins form larger aggregate structures upon encounter with exogenous $\alpha$-synuclein and seed the formation of further misfolding. $\alpha$-synuclein transfer between neighboring cells is not fully understood but can occur via exocytosis secretion into the extracellular medium, resulting in the regulated release of misfolded protein to minimize oxidative and cellular stress. ${ }^{41,42}$ 


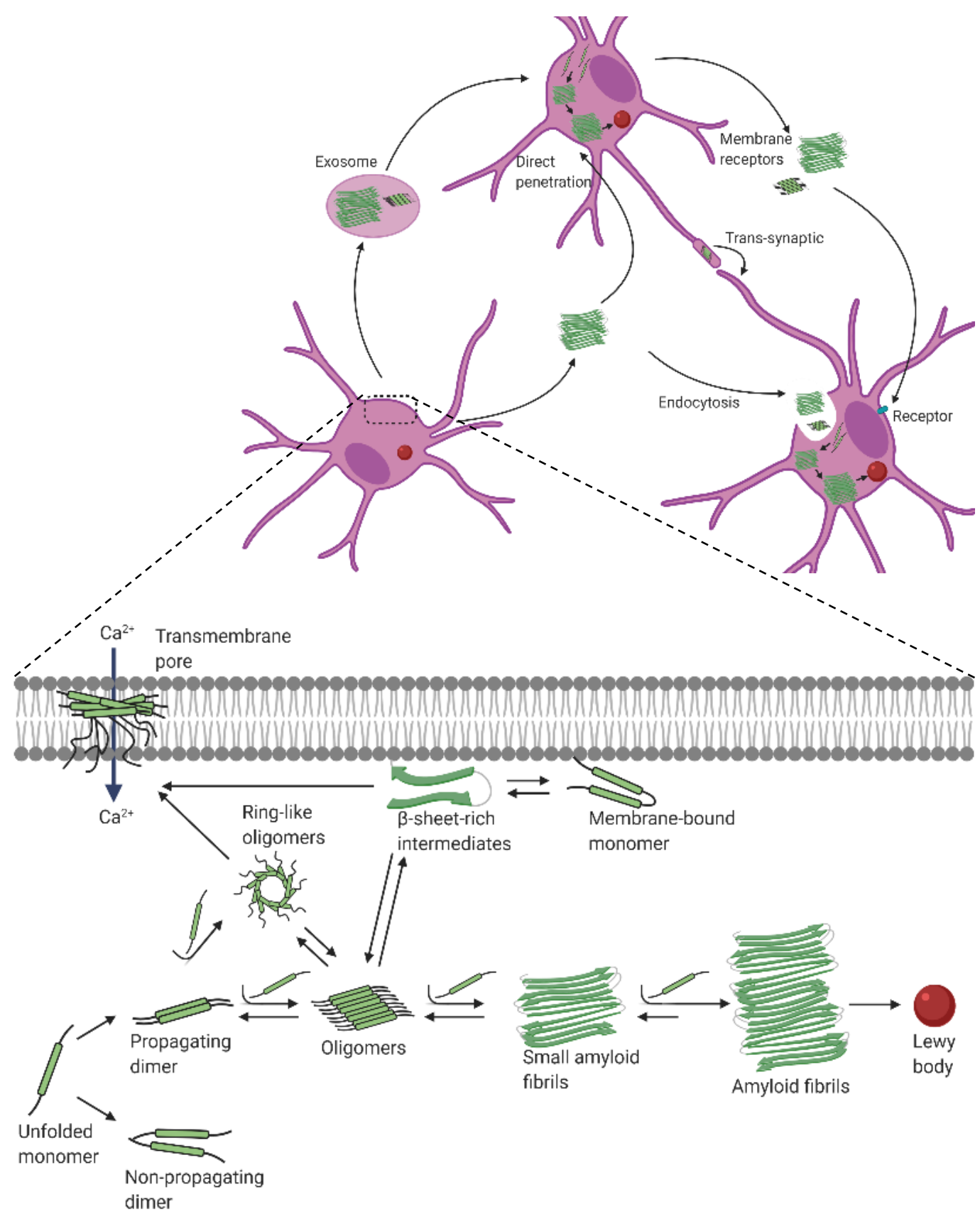

Figure 1. 2: Mechanism depicting various aggregation pathways of monomeric $\alpha$-synuclein and their various influences contributing to neurodegeneration within neuronal cells. Dimerization of monomeric $\alpha$-synuclein progresses into oligomeric species of unique conformations that have the potential to aggregate at a membrane interface or free in cytosolic media in larger fibril complexes. Misfolded $\alpha$-synuclein, oligomers, and protofibrils display seeding ability capable of propagating cell to cell, inducing the misfolding of endogenous $\alpha$-synuclein into larger fibril and LB structures. The prion-like characteristics of $\alpha$-synuclein is a large determinant into the neurotoxicity it inflicts on neuronal cells. Reproduced with permission from Nature. ${ }^{24}$ 


\subsubsection{Neurotoxicity associated with $\alpha$-synuclein}

Various approaches have been performed to examine the dysfunction and toxicity $\alpha$-synuclein can inflict within a neuronal cell. Mutant forms of monomeric $\alpha$-synuclein, specifically A30P and A53T, can lead to the inhibition and impairment of several cellular targets such as the impairment of mitochondrial complex and ubiquitin-proteasomal system. ${ }^{43-45}$ It has been suggested that the formation of an $\alpha$-synuclein-dopamine (DA) adduct is capable of stabilizing monomeric and oligomeric $\alpha$-synuclein species; preventing their progression into a mature fibril. ${ }^{23,46,47}$ An issue stemming from $\alpha$-synuclein-DA adduct formation is compromise of $\alpha$-synuclein's function within neuronal cells, impeding its role on synaptic vesicle regulation and trafficking. An interesting correlation was discussed $^{7}$ where abundance and preferential location of oligomeric $\alpha$-synuclein occurs in dopaminergic neurons due to the stability of dopamine and endogenous catechol species. This could explain why dopaminergic neurons within the substantia nigra are much more vulnerable than other locations in the brain. ${ }^{48}$

\subsubsection{Therapeutic treatment of PD}

Several established treatment options are currently in use in an effort to modulate a dopaminergic response in patients exhibiting PD symptoms while minimizing neurodegeneration. Initial efforts of combating PD symptoms is through the administration of levodopa (L-DOPA), a molecular precursor of DA which is converted within neuronal cells. ${ }^{4,49}$ Due to the specific loss of dopaminergic neurons in PD, the primary role of LDOPA is to replenish DA neurotransmitter and increase synaptic activity. The $60-90$ min half-life of L-DOPA requires the presence of a peripheral decarboxylase inhibitor during 
administration to extend the duration of L-DOPA, preventing its metabolization and enabling L-DOPA to reach its designated area. ${ }^{50}$ The disadvantages of long-term L-DOPA application is the development of reduced response upon drug exposure and dyskinesia coupled with various non-motor symptoms. ${ }^{4}$ Catechol-O-methyltransferase (COMT) and monoamine oxidase inhibitors (MAOIs) are utilized prior to, or simultaneously, with LDOPA to mitigate loss of efficacy and prolong treatment duration. COMT inhibitors prevent the metabolization of L-DOPA to 3-O-methyldopa and increase plasma concentrations $^{4,50}$ while MAOIs prevent L-DOPA enzymatic degradation within the synapse. ${ }^{51}$ Surgical therapy has proven another avenue of PD treatment where deep brain stimulation (DBS) has gained popularity through the application of stimulating electrodes to specific regions of the brain. ${ }^{4}$ This technique is more invasive and expensive than standard treatment options but remains a safer alternative with reduced side effects when compared with chemical intervention.

\subsection{Aptamers}

In 1953, the monumental discovery of the double stranded deoxyribonucleic acid (DNA) structure by Watson and Crick, ${ }^{52}$ and Franklin ${ }^{53}$ paved the way for numerous DNA applications and the eventual fabrication of synthetic oligonucleotides referred to as aptamers. An aptamer is an artificial single stranded oligonucleotide comprised of DNA or ribonucleic acid (RNA) bases that can bind specific targets with high affinity and specificity. There is no significant difference between DNA and RNA aptamers, but DNA aptamers retain greater stability and are not as sensitive to nuclease degradation as RNA aptamers. ${ }^{54}$ Typically synthesized between 20-100 nucleotides in length, aptamers can be 
selected for a broad variety of target structures such as enzymes, small molecules, cells, metal ions and proteins for detection in the range of micromolar $(\mu \mathrm{M})$ to low nanomolar (nM). Aptamers are capable of adopting unique 3D secondary conformations from short stem-loops and hairpins to G-quadruplexes comprised of a tetrad of guanine residues. These complex secondary motifs obtained in aqueous solutions form binding pockets for specific target detection through intermolecular forces such as hydrogen bonding, electrostatic, and $\pi$-stacking interactions.

\subsubsection{Aptamer advantages over antibodies}

Aptamer application as recognition elements within biosensing has gained increasing popularity over conventionally used antibodies due to a number of inherent advantages they possess. ${ }^{55-59}$ 1) Aptamers are selected and synthesized via in vitro processes, enabling them to bind a broader range of targets under tunable conditions. Antibodies are created in vivo through much more expensive and complicated procedures involving live animals where their immune system dictates where the antibody interacts. 2) Synthesis reproducibility, limited batch-to-batch variation, and cost-effective procedures allow aptamers to easily remain within an experimental budget. The relative ease of chemical modification and functionalization allows aptamers to be subjected to a greater range of temperature, ionic and $\mathrm{pH}$ environments without being limited to physiological conditions like antibodies. 3) Aptamers can undergo reversible thermal denaturation and recover their native conformation, leading to an increased stability over extended durations of time. Antibodies, on the other hand, have a significantly reduced shelf life and undergo irreversible thermal denaturation when exposed to increased 
temperature environments. 4) Aptamers can adopt several characteristic secondary conformations that contribute to their specific target binding. Conformational adaptations before and after aptamer-target complexation allow aptamers to discriminate against targets of similar structure without sacrificing their high binding affinity. Due to these unique advantages, aptamers can be utilized as an effective alternative over antibodies in various applications in the areas of drug delivery ${ }^{60}$, molecular therapeutics, ${ }^{61}$ and aptasensor development. ${ }^{62}$ However, aptamer sequences are not without disadvantages as DNAse degradation, renal excretion, and duration of action are several considerations that need to be implemented for their in vivo therapeutic use.

\subsubsection{Aptamer selection process}

The initial conception and development of aptamers was performed independently by two research groups in the 1990s. ${ }^{54,63}$ Tuerk and Gold performed an in vitro selection process known as Systematic Evolution of Ligands by Exponential Enrichment (SELEX) using an RNA library to recognize a specific segment of a T4 DNA polymerase with high affinity. ${ }^{54}$ Ellington and Szostak performed a similar RNA selection process for the specific binding of RNA aptamers with organic dyes. ${ }^{63}$ The SELEX process is an in vitro selection composed of repeating cycles that amplify and identify aptamer sequences with high target binding affinity and is described briefly. The single stranded oligonucleotide library is first synthesized, comprised of up to $10^{15}$ unique aptamer sequence motifs. Each aptamer sequence contains a random centralized region flanked by 5'- and 3'- primer binding regions (PBRs) necessary for PCR amplification at a later stage. Next, the target of interest is incubated with the oligonucleotide library in the desired buffer and temperature 
conditions. Here, individual aptamer sequences exhibiting affinity for the target form an aptamer-target complex through various modes of binding and secondary folding. Targetbound aptamers are isolated from their unbound counterparts and amplified through PCR to enrich the quantity of high-affinity sequences present in the oligonucleotide library for the subsequent SELEX cycle. Typically, 5-15 cycles are performed to generate a sufficient quantity of high-binding sequences from which potential aptamers of interest can be selected for further analysis. Following the SELEX process, the full-length sequence's 5'and 3'-PBRs can be removed as they may not necessarily contribute to aptamer function. Designing truncated forms of the full-length aptamer allow for shorter sequence production times and the formation of minimer sequences. Minimers enable individual segments of the parent strand to be isolated and their respective affinities for the target to be compared to determine which conserved regions are primarily responsible for target interactions. ${ }^{54,63,64}$

\subsubsection{Quantifying aptamer binding affinity $\left(K_{d}\right)$}

Once an aptamer has been selected, it is important to assess its binding affinity to its specified target analyte. The dissociation constant $\left(K_{d}\right)$ is a measure of a target concentration in which 50\% of present aptamers are bound with the target in an aptamertarget complex. ${ }^{65-67}$ This binding equilibrium is determined in terms of molarity and is given by the equation:

$$
A+T \underset{k_{\text {off }}}{\stackrel{k_{\text {on }}}{\rightleftharpoons}} A T
$$

Where $A$ represents the aptamer, $T$ represents the target and $A T$ represents the corresponding aptamer-target complex upon specific binding. When incubated with the 
target, aptamers will exist in an equilibrium state of being bound to the target $\left(K_{o n}\right)$ or unbound from the target $\left(K_{o f f}\right)$. The $K_{d}$ can then be described as:

$$
K_{d}=\frac{[A][T]}{[A T]}=\frac{1}{K_{a}}
$$

When comparing $K_{d}$ values, a lower value represents stronger affinity for the specific target while a higher value indicates substantially weaker target binding. ${ }^{65-67} \mathrm{By}$ maintaining a constant concentration of aptamer or target and titrating increasing concentrations of the opposing ligand into solution, the quantity of bound ligand can be determined through their cooperative binding. Cooperativity is commonly attributed to binding kinetics of enzymes where ligand binding at one saturation site will have a positive or negative impact at additional binding sites, producing a sigmoidal curve indicative of cooperative effects. The sigmoidal curve, resembling an $\mathrm{S}$ shape, will reveal a dramatic increase or decrease in substrate affinity upon initial substrate binding and can be visualized through the application of the Hill-Langmuir equation. The Hill-Langmuir equation describes the extent of cooperative binding between substrate and macromolecule and is commonly used to examine dose-response relationships. ${ }^{68}$

The titration curve expected between ASYN(1-5) and monomeric $\alpha$-synuclein will resemble a hyperbolic curve, indicating that non-cooperative binding has occurred. ASYN(1-5) were designed for their selective binding to monomeric $\alpha$-synuclein at a specific site within its protein sequence and will not influence the binding kinetics of additional ASYN- monomeric $\alpha$-synuclein complex formation. In the event of noncooperative binding, complexation between ASYN and monomeric $\alpha$-synuclein will occur rapidly and linearly as constant concentrations of aptamer are exposed to higher concentrations of monomeric $\alpha$-synuclein. As monomeric $\alpha$-synuclein concentration 
continues to increase the hyperbolic curve will begin to plateau, indicating target site saturation has been achieved. Steady state at high concentrations of monomeric $\alpha$-synuclein will occur as subsequent additions of target will not contribute to a higher binding signal. ${ }^{68}$

There are several factors that can influence an aptamers binding affinity with a target. Various forces are in effect during aptamer-target complexation, and increasing the influence of electrostatic interactions, hydrogen bonding, $\pi-\pi$ stacking, and hydrophobic effect will contribute to an increased sensitivity towards the target structure. Solvent effects must also be considered when characterizing aptamer $K_{d}$ values. Water molecules along the interfaces of a protein target may induce conformational changes along the protein surface and influence interactions with the aptamer. ${ }^{69}$ In one instance, water molecules were identified as being an active component in the aggregation of self-assembled amyloid fibrils. ${ }^{70}$ Numerous experimental methods can be used for characterizing aptamer $K_{d}$ such as ITC, CD, PAGE, SPR, and UV-Vis.

\subsection{Aptamer characterization techniques and biosensor designs}

\subsubsection{Electrochemistry}

Electrochemistry encompasses a large area of chemistry primarily focused on the electron transfer reactions that occur at an electrode/electrolyte interface when immersed in an electrolytic solution. ${ }^{71,72}$ These electron transfer reactions can be described through thermodynamics and kinetics where thermodynamics revolves around the enthalpic and entropic changes within a reaction while kinetics describes the reaction rate. An electrode providing electrons to the electrolyte is referred to as the cathode while the electrode that 
accepts electrons from the electrolyte is referred to as the anode. This process can be described in the following reaction:

$$
O+n e^{-} \rightleftharpoons R
$$

Where $O$ represents the oxidized species, $n$ represents the number of electrons transferred within the reaction, and $R$ represents the reduced species. In the absence of an external driving force the reduction-oxidation (redox) reaction is at equilibrium with equivalent forward and reverse reaction rates. ${ }^{71,72}$ Through the application of external energy to the electrochemical system the reaction can be driven in a desired direction, allowing the measure of Faradaic electron charge transfer. Faradaic charge transfer can be described as the transfer of electrons across an interface that involves the oxidation and reduction of an electroactive species. Non-Faradaic electron transfer comprises the physical adsorption and desorption processes that occur at the electrode/electrolyte interface as a function of potential accumulation. ${ }^{73}$ An electrochemical biosensor incorporating aptamers, termed aptasensor, was coupled with a three-electrode electrochemical cell consisting of respective working, counter, and reference electrodes connected to a potentiostat power supply (Figure 1.3).

A potential is applied by the potentiostat where electrons flow between the reference and working electrodes with respect to an opposing potential applied between the counter and working electrodes respectively. ${ }^{71,72}$ This enables electron transfer to occur between the counter and working electrodes, while the electrochemical reactions at the working electrode can be compared directly to the constant potential of the reference electrode. 


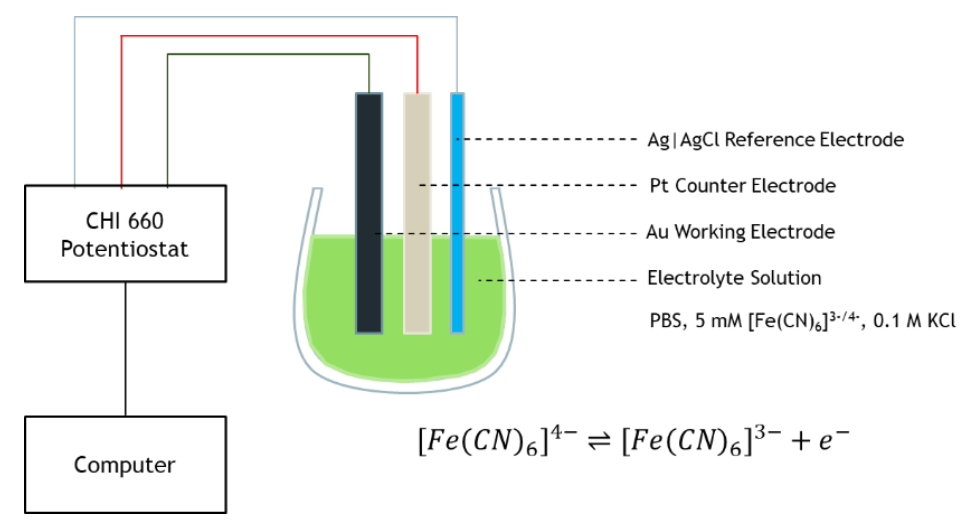

Figure 1. 3: A $10 \mathrm{~mL}$ electrochemical cell consisting of an $\mathrm{Ag} \mid \mathrm{AgCl}$ reference electrode, platinum counter electrode, and a gold working electrode connected to a CHI660 potentiostat. CV and EIS measurements were performed in an electrolyte consisting of $5 \mathrm{mM}\left[\mathrm{Fe}(\mathrm{CN})^{6}\right]$.

At equilibrium, the kinetics of the electrochemical reaction can be attributed to the mass transport of ions via bulk migration and diffusion. Mass transport is where the electroactive species approaches the electrode interface from the bulk solution until it encounters a region that influences adsorption desorption processes to occur. Migration occurs when an electroactive species movement is influenced by an external electrical field that attracts the opposing charged species. Diffusion processes occur as a species moves down its concentration gradient in solution. This process is entropically driven as the species attempts to uniformly disperse within the electrolyte. ${ }^{71,74}$

A biosensor utilizes a sensitive biological recognition device to measure the physical, chemical, or electrical signals of a reaction and convert them into a quantifiable signal. ${ }^{75,76}$ The foundation of a biosensor is comprised of several essential components: a biorecognition element, a transducer element, and a signal processing element. ${ }^{75,77}$ Biorecognition elements throughout literature primarily revolve around DNA/RNA aptamers, antibodies, peptides, and enzymes. These bioreceptors are often immobilized on the interfaces of transducer elements and are responsible for interacting with the analyte of 
interest. Changes that occur as a result of these interactions take various forms such as conformational and mass changes, production of light and heat, or the transfer of electrons resulting in a species reduction or oxidation. The specificity and selectivity of the bioreceptor dictates the biosensors ability to effectively detect the target upon exposure, or amidst a congregation of many species. The transducer element quantifies alterations within the interface environment and converts this change into a signal that can be readily processed and observed through the application of a signal processing element. ${ }^{78,79}$ Aptasensors, in particular, exploit aptamers as the biorecognition element to analyze the specific binding event that occurs between a target and its selected aptamer. Aptasensors offer numerous advantages, making them inherently attractive for a wide range of experimental procedures. An aptasensors ability for specific and reproducible detection, continuous data collection, rapid response times, low cost, and ease of experimental preparation have contributed to its rise in popularity and applicability over recent years. ${ }^{80}$ Despite aptasensor development in a wide range of fields, they can be grouped into two main categories for use in electrochemical and optical applications. ${ }^{80,81}$

Electrochemical biosensors are readily available throughout commercial applications such as in health monitoring,${ }^{82}$ security,${ }^{83}$ environmental and food safety control. ${ }^{84}$ The general principle of electrochemical aptasensing is to analyze the electrical reactions that occur between a surface-immobilized aptamer and a target that results in the consumption or release of electrons. A resulting current or potential difference will occur across the interface that will create a signal that can be measured. An electroactive species is usually applied to the sensing system to increase the rate of electron transfer between the electrolyte solution and sensing platform. ${ }^{74}$ There are several categories of electrochemical 
sensing such as potentiometric, amperometric, voltammetric, and impedimetric sensing, with emphasis placed on cyclic voltammetry $(\mathrm{CV})$ and electrochemical impedance spectroscopy (EIS).

When a linear potential range is applied to the counter and working electrode and

the corresponding current signal measured, this is referred to as voltammetry. ${ }^{85}$ Cyclic voltammetry is one of the most widespread electrochemical techniques where a linear potential is scanned from point $E_{0}$, where no electron transfer occurs, to point $E_{l}$ where it is reversed and scanned back to $E_{0}{ }^{86}$ As the current is increased over the standard potential $E^{o}$, species diffuse towards the electrode interface where oxidation or reduction processes occur. The surface current reaches a cathodic peak $\left(i_{c}\right)$ at $E_{a}$ when the diffusion of ions decreases, and the electroactive species cannot maintain a constant rate of electron transfer across the interface. As the current is reversed, the previously oxidized species is now reduced, resulting in an anodic peak $\left(i_{a}\right)$ at $E_{c .}{ }^{86}$

EIS is a powerful method of characterizing the electrical properties and interfacial reactions that exist at the electrode-electrolyte interface. Since its conception, EIS has been used in numerous areas to study the effects of electrode kinetics, batteries, corrosion, and biochemical reactions and exchange mechanisms. The basis of EIS involves the measure of impedance at an interface by superimposing an AC potential and recording the current output signal. More details regarding this technique are provided in section 1.3.1.2.

\subsubsection{Aptamer immobilization on a solid surface}

When fabricating an aptasensor via aptamer immobilization to a solid substrate surface, various parameters and methods of immobilization can be considered depending 
on the nature of the aptasensor. The method of immobilization will directly affect the measured $K_{d}$ of the aptamer-target interaction as immobilization may alter its conformational freedom and stability in solution. If the aptamers mobility is compromised compared to its native uncoiled state in solution, its affinity and binding specificity to the targets active site may be hindered or enhanced accordingly. For example, upon an environmental change, such as ionic concentration or target presence, electrostatic repulsion within the aptamer will be neutralized, allowing hydrogen bonding to fold into a more stable secondary structure. For the purposes of this thesis, a gold planar electrode formed the foundation of the electrochemical aptasensor while gold nanoparticles (AuNPs) formed the basis of the optical biosensor.

Several methods of aptamer immobilization are commonly used throughout literature, with physical adsorption, covalent bonding, and self-assembled monolayers (SAMs) being the most common. ${ }^{87}$ Physical adsorption of aptamers onto a transducer surface is the easiest and most cost efficient as it does not require the use of additional modifications within the aptamer sequence. Physical adsorption was the primary immobilization method onto the AuNPs surface, and largely depends on the physicochemical properties of the individual DNA bases. ${ }^{88}$ Figure 1.4 illustrates the heterocyclic structures of the individual DNA bases and their respective binding interactions to an exposed gold surface.

The buffer environment in which physical DNA adsorption occurs is regulated at a neutral 7.0-7.4 pH, resulting in the DNA having a negatively charged phosphate backbone. Altering the environment outside of these boundaries will cause the protonation or deprotonation of the base ring nitrogen, providing additional stabilization through 
increased electrostatic charge. Base hydrophobicity will also contribute to aptamer-metal interactions and its orientation along the metal surface. Various studies have been performed on base orientation along a metal surface where it was found that DNA predominantly adopts a random orientation with respect to tilted or parallel binding angle. ${ }^{89-91}$ It has been suggested that adenine forms the strongest interaction with an AuNP through the use of its N7 base ring nitrogen and exocyclic amino group. ${ }^{90,92}$ The main drawback of physical adsorption is that it is difficult to control the orientation of the aptamer bound to the surface as it will tend to adopt a random conformation to minimize repulsive effects from adjacent DNA sequences.
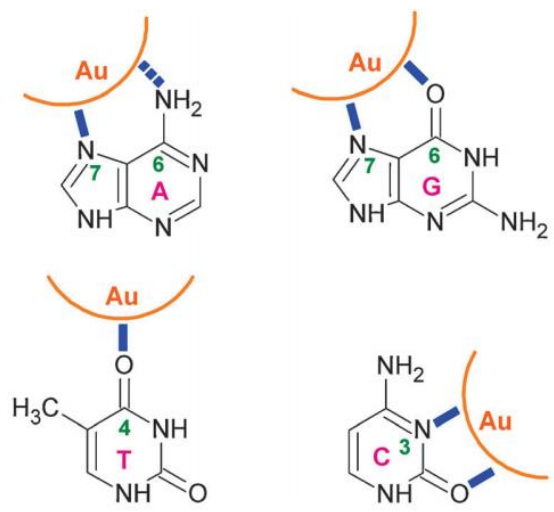

Figure 1. 4: Non-specific binding of DNA bases (adenine, guanine, thymine, cytosine) to an exposed gold surface. Used with permission from Royal Society of Chemistry. ${ }^{88}$

DNA immobilization on planar gold surfaces through the use of a terminal-thiol modification is a common approach for developing biosensing apparatus. ${ }^{93,94}$ Typically, a disulfide modification will be positioned at the 5'- or 3'-terminal of the aptamer, where incubation in the presence of a reducing agent such as TCEP or DTT will cleave the disulfide and release the thiolated aptamer. Covalent bonding will occur spontaneously between the thiol-functionalized aptamer and the gold surface. ${ }^{95}$ The sulfhydryl group 
(RSH) becomes deprotonated $\left(\mathrm{RS}^{-}\right)$and forms a covalent bond to the gold surface while RSH forms through weaker coordination bonds using the sulfur lone pair electrons. ${ }^{96}$

There are several advantages to anchoring an aptamer via a thiol modification. A linker or spacer is typically inserted between the thiolated terminal and the aptamer sequence to provide more conformational freedom and to minimize the possibility of nonspecific interactions from the nitrogenous bases. Alkane-thiols are historically used as they are flexible, highly ordered, and chemically stable in solution, allows the aptamer to adopt a more natural binding orientation upon target binding. ${ }^{93,97,98}$ Poly-thymine residues have been used as an alternative spacer as they retain a significantly lower chance of binding the gold surface due to their lack of available binding sites. ${ }^{99}$

A SAM is the spontaneous ordered adsorption and orientation of a chemical species onto a substrate surface. ${ }^{87,97,98,100}$ The monolayer component is typically comprised of a long chain molecule, in this case the aptamer functionalized with the terminal alkane-thiol, with a polar head group, being the sulfur atom. The resulting monolayer forms a dense, uniform structure perpendicular to the metal surface. ${ }^{95}$ Gold is a classic material for thiol SAM fabrication as it is inert to most chemical species and is relatively available. ${ }^{101}$ Selfassembly offers many attractive advantages in biosensing due to monolayer reproducibility and stability, and the lack of non-specific interactions between the immobilized aptamer and the gold surface.

\subsubsection{Types of aptasensor}

Two main categories of electrochemical aptasensors are configurational and conformational-based aptasensors, both comprised of unique methods of fabrication and 
signal analysis in the presence and absence of target. Configurational aptasensors center around the induced association or dissociation of the aptasensor upon target binding where the chemical behavior at the electrode interface is significantly varied.

A paper by Fan et al. ${ }^{102}$ in 2003 described one of the first binding-induced aptasensors utilizing a molecular beacon aptamer (MAB) immobilized on an electrode surface. MABs typically consist of a stem-loop structure containing a fluorescent probe or quencher attached to either terminal on the aptamer sequence. ${ }^{103}$ In this case, the immobilized aptamer was tethered to a gold electrode surface through Au-S covalent bonding from the 3'-hexamethylene thiol and the planar gold surface while the 5'-terminal contained a redox active ferrocene modification. In the absence of a complementary DNA strand, the MAB adopted a stem-loop conformation that brought the 5'-ferrocene moiety into close proximity with the electrode surface. This is an example of a "signal off" biosensor where the ferrocene's proximity to the electrode surface readily allows electron transfer to occur. Upon introduction of the complementary DNA strand, hybridization commenced where the initial stem-loop conformation was disrupted and replaced by a more linear, thermodynamically stable double stranded helix structure. This structural transformation separates the ferrocene from the electrode surface, significantly reducing distance dependent electron transfer. Using $\mathrm{CV}$, the authors established a hybridization limit of detection (LOD) of $10 \mathrm{pM}$ as a function of the change in electron transfer.

Within the last year, Taghdisi et al. ${ }^{104}$ designed an $\alpha$-synuclein-induced disassembly mechanism based on the competitive binding of a target-specific DNA strand over its respective complementary strand. Using an oligomeric $\alpha$-synuclein aptamer designed by Tsukakoshi et al., ${ }^{105}$ its complementary strand was immobilized to a gold 
electrode surface through a 5'-terminal thiol modification to which the $\alpha$-synuclein binding aptamer was hybridized. Exonuclease I (Exo I) enzymes were added to the hybridized system that are capable of selectively hydrolyzing terminal phosphodiester bonds within single-stranded DNA sequences. ${ }^{106}$ Addition of Exo I to the dsDNA in the absence of oligomeric $\alpha$-synuclein has zero effect as the dsDNA are protected against digestion. Terminal deoxynucleotidyl transferase (TdT) was then added to the aptasensor as a means of elongating the 3 '-aptamer terminal through polyT generation. ${ }^{107} \mathrm{TdT}$ is a templateindependent catalyzed reaction that has been commonly used in nucleic acid detection sensors. ${ }^{108} \mathrm{TdT}$ generates polyT sequences along the 3'-terminals of both hybridized DNA sequences, increasing their length along the electrode surface. The redox agent methylene blue (MB) is a positively charged molecule that can interact with ssDNA through interactions with its negatively charged phosphate backbone, and dsDNA by intercalating between DNA bases and stabilized via $\pi$-stacking. ${ }^{109}$ Binding of MB to the extended DNA sequences leads to an observable increase in current response as a result of more efficient charge transfer along the electrode interface. Addition of oligomeric $\alpha$-synuclein and Exo I induces conformational change and displacement of the hybridized aptamer, forming an aptamer-target complex that reveals the immobilized strand for Exo I digestion. TdT addition catalyzes 3'-polyT extension of only the 3' complementary strand, leading to a decrease in electrochemical signal. The oligomeric aptasensor had a linear dynamic range (LDR) or $60 \mathrm{pM}$ to $150 \mathrm{nM}$ and an LOD of $10 \mathrm{pM}$.

Conformational change aptasensors are much simpler in general, solely relying on a target-induced structural change within the aptamer sequence and minimizing the necessity for modifications and multiple fabrication steps. Xiao et al. ${ }^{103}$ constructed a 
simple electronic aptamer-based sensor for the detection of thrombin by covalently modifying the 3'-terminal with MB and immobilizing a G-quadruplex binding aptamer to a gold electrode. In the absence of thrombin, the unfolded aptamer retained enough flexibility to bring $\mathrm{MB}$ in close proximity to the gold electrode for electron transfer to occur. Thrombin addition induced folding of the sequence into a G-quadruplex that hindered electron transfer from solution and displaced the $\mathrm{MB}$ redox probe from the electrode surface. This strategy also incorporated a regeneration step through aptasensor immersion in $6 \mathrm{M}$ guanidine, allowing the aptasensor to be reusable and yielding an LOD of $6.4 \mathrm{nM}$.

\subsubsection{Electrochemical impedance spectroscopy (EIS)}

Resistance (R) is defined as the ability of a sensing element to resist the flow of electrons and determined using Ohm's law in terms of the ratio between the systems potential (E) and current (I):

$$
R=\frac{E}{I}
$$

Impedance (Z) corresponds to the resistance of a system to AC current at a specified frequency as a function of its resistive and capacitive components. When applied to the system, the potential excitation signal $\left(E_{t}\right)$ is sinusoidal and expressed as a function of time $(t):^{74}$

$$
E_{t}=E_{o} \sin (\omega t)
$$

Where $E_{o}$ represents the initial excitation potential amplitude and $\omega$ represents the radial frequency:

$$
\omega=2 \pi f
$$


Where $f$ is expressed in $\mathrm{Hz}$ and $\omega$ is expressed in $\mathrm{rad} / \mathrm{s}$. The excitation potential will yield a current response $\left(I_{t}\right)$ as a function of time $(t):{ }^{74}$

$$
I_{t}=I_{o} \sin (\omega t+\varphi)
$$

Where $I_{o}$ represents the initial current amplitude and $\varphi$ is the phase angle of the current output with respect to the potential input. Since the impedance is analogous to resistance, we can utilize Ohm's law to determine the impedance of the system:

$$
R=Z=\frac{E_{t}}{I_{t}}=\frac{E_{o} \sin (\omega t)}{I_{o} \sin (\omega t+\varphi)}=Z_{o} \frac{\sin (\omega t)}{\sin (\omega t+\varphi)}
$$

Euler's formula describes circular motion in terms of its real $\left(Z^{\prime}\right)$ and imaginary $\left(Z^{\prime \prime}\right)$ components and can be applied for analysis of points along a unit circle according to their phase angle $\varphi$ :

$$
\exp (j \varphi)=\cos \varphi+j \sin \varphi
$$

Applying Euler's formula to the previous impedance formula, it can now be converted into a complex expression as a function of its potential and current responses: ${ }^{74}$

$$
\begin{aligned}
& E_{t}=E_{o} \exp (\mathrm{j} \omega \mathrm{t}) \\
& I_{t}=I_{o} \exp (j \omega t-j \varphi) \\
& Z(\omega)=\frac{E_{t}}{I_{t}}=Z_{o} \exp (j \varphi)=Z_{o}(\cos \varphi+j \sin \varphi)
\end{aligned}
$$

When visualizing the impedimetric data, it is commonly formatted into a Nyquist plot where the imaginary impedance component $\left(Z^{\prime \prime}\right)$ is plotted with respect to the real impedance component (Z’) (Figure 1.5). The impedance vector $|Z|$ represents the modulus of the complex number obtained previously and the argument of $|Z|$ forms between the impedance vector and the $Z$ '-axis, corresponding to the phase angle of the $Z$ ', ${ }^{110,111}$ 


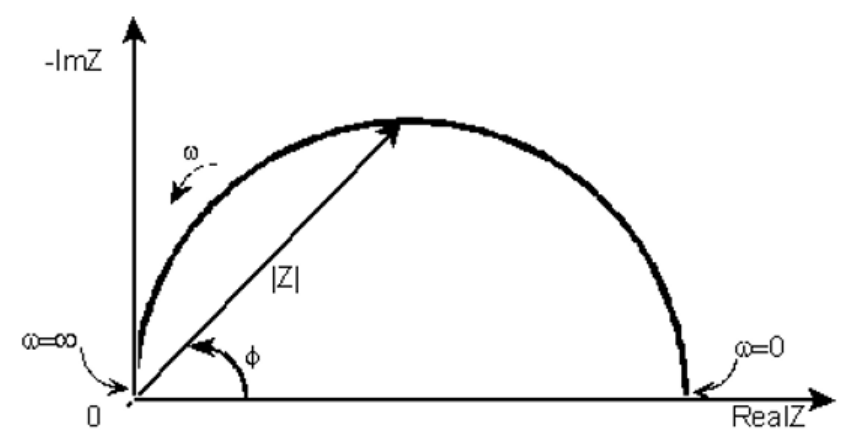

Figure 1. 5: Electrochemical impedance spectroscopy (EIS) is represented through a Nyquist diagram.

As the AC excitation potential is applied to the system over a specified frequency range, the $\mathrm{AC}$ current response is analyzed and depicted as a semicircle increasing from the right side of the Nyquist plot $(\omega \rightarrow 0)$ to the left side $(\omega \rightarrow \infty)$. The electrical components of an electrochemical system being the resistor, capacitor, inductor, and angular frequency, determine the respective impedance measured. The results of the Nyquist plot are fit to an electrochemical equivalent circuit that consists of a resistance $\left(R_{S}\right)$ in series with a parallel resistance $\left(R_{c t}\right)$ and capacitor $\left(C_{d l}\right)$. Inductance effects are not considered in the electrochemical measurements due to its negligible contributions. ${ }^{12}$

The resistance in series is equivalent to the system impedance where $R_{s}$ refers to the solution resistance within the electrolyte, remaining independent of the frequency and retaining only real components. It remains in phase with the potential as it passes through the resistor, therefore only contributing to the movement along the $\mathrm{x}$-axis and having a value of zero for the imaginary component. Regarding $R_{2}$ and the capacitor in parallel, these values refer the Faradaic charge transfer resistance that occurs at the interface as the electroactive species engages in redox reactions and to the double layer capacitance experienced along the electrode-electrolyte interface respectively. $R_{c t}$ is a direct measurement of the impedance experienced along the electrode interface as electrons are 
impeded across the metal plane. ${ }^{110}$ The double layer capacitance is a result of the electroactive and ionic species within the electrolyte adsorbing to the electrode surface (Figure 1.6).
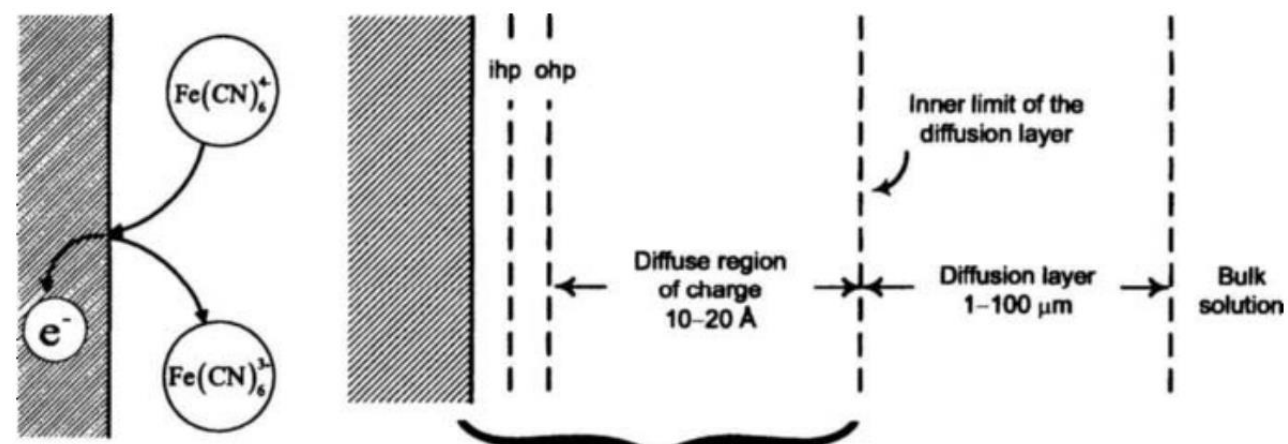

Figure 1. 6: Illustration depicting the diffusion of electroactive ions in solution and the associated redox chemistry at the gold electrode-electrolyte interface. Ferrocyanide is oxidized to ferricyanide through Faradaic charge transfer that can be measured using EIS and CV techniques. A schematic of a double layer at the gold electrode surface in the presence of the $\mathrm{Fe}(\mathrm{CN})_{6}{ }^{3-4-}$ electroactive species. Used with permission from Wiley. ${ }^{111}$

Along the metal surface, electron accumulation causes an electroactive species to specifically adsorb to the surface, forming the boundary of the Inner Helmholtz plane (IHP). The Outer Helmholtz plane (OHP) is formed from the closest approach of solvated ions to the electrode surface that still reside within the electrolyte. Beyond the OHP lies the diffusion region where ionic species near the electrode surface contribute to the overall charge of the electrolyte before diffusing into the bulk solution. ${ }^{74}$

The electrochemical capacitance is strictly dependent on the imaginary components as the input potential and output current response are out of phase. As the frequency is increased, the impedance experienced along the capacitor is subsequently decreased as a result of low- and high-frequency limitations. Low frequencies occur in the region where $\omega \rightarrow 0$ and exist as a linear slope referred to as Warburg impedance $\left(Z_{w}\right)$. This region represents the diffusion of adsorbed ions back into the electrolyte from the electrode 
surface. The low-frequency linearity of this region represents the diffusion process that occurs within the electrochemical system. ${ }^{74}$ As the frequency is increased, the importance of $Z_{w}$ decreases and both $R_{c t}$ and $C_{d l}$ increases respectively. At high frequencies, the capacitance impedance is negligible as electron charge is conducted easily and relies solely on the value of $R_{s}$. In the mid-low frequency ranges, the capacitance impedance increases as current flows primarily through $R_{c t}{ }^{74}$ The higher the value of $R_{c t}$, the larger the diameter of the semicircle within the Nyquist plot.

\subsubsection{Optical assays and techniques}

Optical aptasensors have been developed for various target structures from proteins, metal ions and nanoparticles, small molecules, and amino acids. ${ }^{113}$ The two most commonly applied optical aptasensors center around fluorescence and colorimetric sensing which rely on visual signals correlating to the binding event occurring within an aptamertarget complex. Quantum dots (QDs), molecular dyes, and fluorescent proteins are examples of fluorescent biosensors used throughout literature. Regarding aptasensors in particular, fluorescent-label modifications can be directly synthesized within the oligonucleotide sequence. Aptamer-based molecular beacons utilize the induced conformational change of an aptamer when it associates with its specified target to produce a quantifiable fluorescent signal. Incorporating fluorophore-quencher labels within an aptamer sequence possessing a specific 3D geometry such as a hairpin or G-quadruplex is the general premise of fluorescent aptasensors. The distance between the fluorophorequencher pair is largely based on the induced conformational change experienced upon target binding. ${ }^{114}$ 
AuNPs host a broad range of characteristic properties that have been exploited for use in various fields surrounding their physical, chemical, electronic, and optical applications. ${ }^{115}$ In particular, AuNPs have been commonly applied to optical biosensors for their visually distinct colours as a function of their size dependence. ${ }^{116}$ Displaying a vibrant red colour as a monodisperse nanoparticle, their aggregation induces a gradual colour change from red to purple and ultimately a deep blue due to the shift in localized surface plasmon resonance.

A surface plasmon is the collective oscillation of charge density at the interface of a noble metallic surface, arising from the oscillation of conduction band electrons. ${ }^{117-119}$ Therefore, SPR is defined as the excitation of these electrons through their interaction with an electromagnetic wavelength of a specific frequency, causing its propagation along the metal-dielectric medium interface. ${ }^{120,121}$ This interaction polarizes the surface electrons and induces alternate charges to accumulate on opposing ends of the nanoparticle. ${ }^{122}$ Instances where photons interact with conduction band electrons along the metallic interface can lead to its absorption, resulting in the visually appealing colour changes occurring in AuNP biosensors. In the event of an electromagnetic wave interacting with a nanoparticle of smaller size, the charge density across the metallic surface oscillates in sync with the electromagnetic wave and can be observed within UV-Vis spectra. ${ }^{123}$ The nanoparticles size, shape, local environment, and interparticle distance all contribute to localized surface plasmon resonance (LSPR). ${ }^{124}$ As the AuNP size increases, the refractive index of the metallic interface becomes altered and the corresponding absorption intensity increases. As a wavelength of light is absorbed at the plasmon resonance frequency on the nanoparticle surface, the incident beam is scattered at a different absorbance intensity. ${ }^{122}$ 
This leads to a red-shifted maximum observable in the visible range from $525 \mathrm{~nm}$ to 625 nm. ${ }^{125}$ Although gold displays broader and less intense LSPR absorption than silver nanoparticles (AgNPs), its chemical stability has led to its regular use in biosensing. ${ }^{126}$

Colorimetric aptasensors where the characteristic visual changes of AuNPs are exploited during target or salt induced aggregation and dispersion have been developed to quantify the specific detection of a target. One example was performed by Wang et al. ${ }^{127}$ where a colorimetric aptasensor was developed for the specific detection of the pesticide omethoate. Single stranded DNA aptamers were non-specifically adsorbed to the surfaces of AuNPs, stabilizing and dispersing them in solution. In the presence of $\mathrm{NaCl}$, the aptamers protected the AuNPs and inhibited salt-induced aggregation. When the pesticide target was added with the aptamer-AuNP sensor, specific binding between the aptamer and the target induced a conformational change within the aptamer causing it to preferentially release from the AuNP surface. The loss of the aptamer exposed the surface of the AuNP, allowing the high $\mathrm{NaCl}$ concentration to overcome the initial resistive forces and aggregate the AuNPs. The changes in aggregation are easily distinguished between red and blue, symbolizing dispersed vs aggregated AuNPs respectively. This change in colour could then be quantified via UV-vis spectroscopy to quantitatively determine the concentration of target present. $^{128}$

\subsubsection{UV-Vis characterization of DNA structure}

Ultraviolet absorption by DNA is well documented as it has been shown to not only induce DNA damage ${ }^{129}$ but can also cause an electronic change within its molecular structure. Upon energy absorption excitation can occur, causing electrons to transfer from 
the lower energy ground state to a higher energy excited state. As light encounters DNA, it gets absorbed and provides a decreased transmitted light intensity that is described through the Beer-Lambert equation: ${ }^{71,130}$

$$
I=I_{o} 10^{-\varepsilon c l}
$$

Where $I$ is the transmitted light intensity, $I_{O}$ is the initial light intensity, $\varepsilon$ is the molar extinction coefficient in $\mathrm{M}^{-1} \mathrm{~cm}^{-1}, c$ is the molar concentration, and $l$ is the path length $(\mathrm{cm})$. The total absorbance can be written as

$$
\begin{aligned}
& A=\log \left(\frac{I_{o}}{I}\right) \\
& A=\varepsilon c l
\end{aligned}
$$

The absorbed energy depends on the difference between the ground and excited states; a larger difference between energy levels will translate to a smaller absorption value. When UV light is absorbed, the excited electron transitions from the highest occupied molecular orbital (HOMO) to the lowest unoccupied molecular orbital (LUMO). A chromophore is composed of a group of atoms that are responsible for absorbing light at a specific frequency. ${ }^{71,130}$ As the structural conformation of the chromophore changes, the intensity of light absorption changes accordingly. UV-vis is commonly used for quantifying DNA concentrations as the purine and pyrimidine bases have a maximum absorbance around $260 \mathrm{~nm}\left(\mathrm{~A}_{260}\right)$. Denatured DNA elicits higher absorbance (hyperchromicity) at $260 \mathrm{~nm}$ than duplex DNA. ${ }^{131,132}$ Thus, an increase in absorbance with temperature can be related to the unzipping of a duplex structure. Alternatively, Gquadruplex motif chromophore absorbs weakly at $295 \mathrm{~nm}$ and the loss of this absorbance leads to a lower absorbance (hypochromicity) when it is denatured. With increasing temperature, the G-quadruplex structure is lost and an increase in absorbance is observed 
as a result of DNA denaturation. ${ }^{131}$ Melting temperature $\left(T_{m}\right)$ analysis involves subjecting DNA to a ramping temperature gradient where analysis at $260 \mathrm{~nm}$ and $295 \mathrm{~nm}$ helps unravel the structural features within the sequence. Sequences expressing a higher melting temperature, indicated by an inflection point, have a more stable structure and are more ideal for therapeutic applications.

\subsubsection{Circular dichroism (CD)}

Circular dichroism (CD) is a method commonly used for measuring the secondary conformation of nucleic acids and proteins when exposed to left- and right-circularly polarized light. ${ }^{133}$ Polarized light is described as the sum of two perpendicular electromagnetic waves where both components are in phase. When one of these components becomes out of phase, the corresponding wave adopts a helical orientation and is circularly polarized in the left- or right-handed direction. The left- and right-circularly polarized waves are non-superimposable images of one another, exhibiting a chirality that

allows them to study the chiral nature of a biological structure. ${ }^{134}$ Depending on the molecular asymmetry present within the secondary structure, its interactions with left- and right-circularly polarized light will be different. Combining the two left- and right-handed components gives an elliptical shape that relates the absorbance of the optically active compound to the incident wavelength. ${ }^{133}$

CD spectra can be broken into two components: the far-UV region $(190-250 \mathrm{~nm})$ and the near UV region $(250-350 \mathrm{~nm})$. The far- $\mathrm{UV}$ region is used to measure the secondary structure within a protein in terms of an $\alpha$-helix, $\beta$-sheet, or a random, uncoiled protein. ${ }^{135}$ Each of these protein structures will provide characteristic peaks as a function of the 
electronic transitions within its peptide backbone, which is the predominant chromophore. ${ }^{133}$ Several types of electronic transitions can occur within the amide backbone. The $n \rightarrow \pi^{*}$ transition is deemed the weakest as they occupy nodal planes that are perpendicular to one another. This type of transition is forbidden electronically, and results in a weak elliptical signal in the range of $210-220 \mathrm{~nm}$ as the non-bonding electrons within a carbonyl are excited. ${ }^{134}$ The $\pi \rightarrow \pi^{*}$ transition also occurs within the carbonyl group and requires significantly more excitation energy, resulting in a substantially more intense absorbance in the $190 \mathrm{~nm}$ range. ${ }^{134}$ Figure 1.7 illustrates the unique CD spectra of each of these protein secondary structures.

The $\alpha$-helical structure retains positive ellipticity at $191 \mathrm{~nm}\left(\pi \rightarrow \pi^{*}\right)$ and negative ellipticity at $208 \mathrm{~nm}\left(n \rightarrow \pi^{*}\right)$ and $222 \mathrm{~nm}\left(\mathrm{n} \rightarrow \pi^{*}\right)$, the $\beta$-sheet has a positive signal 196 $\mathrm{nm}\left(\pi \rightarrow \pi^{*}\right)$ and a negative signal at $218 \mathrm{~nm}\left(n \rightarrow \pi^{*}\right)$, and the uncoiled protein has positive ellipticity around $210 \mathrm{~nm}\left(n \rightarrow \pi^{*}\right)$ negative ellipticity around $200 \mathrm{~nm}(\pi \rightarrow$ $\left.\pi^{*}\right){ }^{135}$

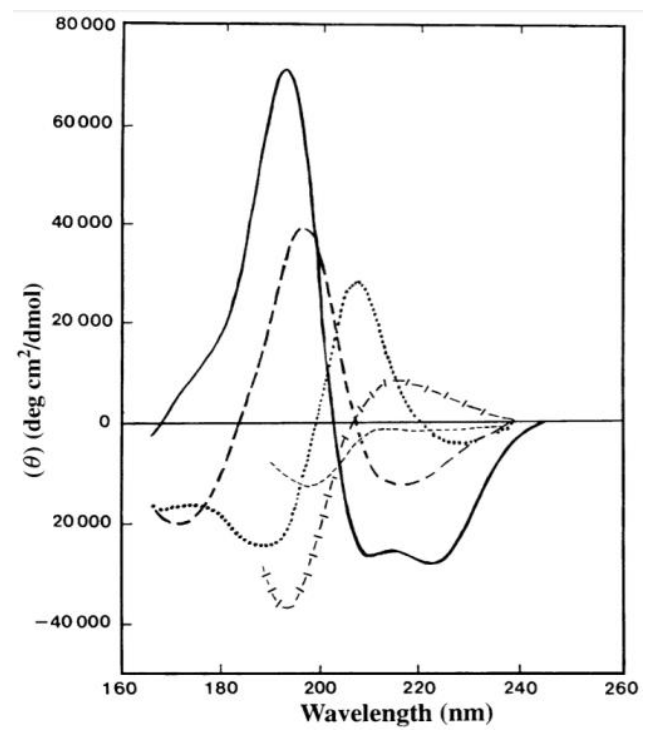

Figure 1. 7: CD spectra differentiating $\alpha$-helix (solid line), $\beta$-sheet (long-dashed line), and random coiled (short-dashed line) protein secondary structures. Used with permission from Wiley. ${ }^{134}$ 
Unless a protein contains explicit tertiary structure such as aromatic or disulfide residues, it will have minimal effect on the absorbance within the near UV region. ${ }^{135}$ DNA conformation can be characterized in this region at high sensitivities and provide an abundant amount of data regarding its secondary structure in both the absence and presence of a specific target. In DNA, the nucleoside bases have similar electronic structure and act as chromophores where electronic transitions occur upon UV light exposure. ${ }^{136}$ Due to the planar nature of DNA bases, asymmetry arises from the deoxyribose sugar residues along the DNA backbone. DNA base structure is composed of extensive $\pi$-interactions where there are many occupied and unoccupied $\pi$ orbitals that can undergo $\pi \rightarrow \pi^{*}$ transitions along with nonbonding electrons $n \rightarrow \pi^{*}$ transitions. These electronic excitations predominantly occur within the nonbonding electrons of nitrogen of proteins, but can also be observed within the nonbonding electrons of the carbonyl group of the DNA backbone. ${ }^{136}$

DNA commonly adopts a B-form conformation ${ }^{137}$ and its respective CD spectra depends largely on its base composition. B-form DNA has two constituent peaks, a negative signal at $240-260 \mathrm{~nm}$ and a positive signal at $260-280 \mathrm{~nm} \cdot{ }^{137} \mathrm{~A}$-form structure is commonly associated with RNA as a result of its ribose sugar being restricted into an C3' endo puckering orientation. ${ }^{138}$ DNA is capable of adopting both A and B forms, as well as B-A transitions depending on its environment. ${ }^{139}$ A-form DNA retains the different CD characteristics with a negative $210 \mathrm{~nm}$ peak and a dominant positive $260 \mathrm{~nm}$ peak. Guanine-rich DNA structures are capable of forming a G-quadruplex secondary structure that can be parallel or anti-parallel oriented (Figure 1.8) Parallel G-quadruplex structures contain positive bands at $210 \mathrm{~nm}$ and $260 \mathrm{~nm}$ with a negative band at $240 \mathrm{~nm}$, while anti- 
parallel G-quadruplex structures contain shifted bands with positive ellipticity at $210 \mathrm{~nm}$ and $290 \mathrm{~nm}$ with negative ellipticity at $260 \mathrm{~nm}$. Base stacking within a G-quadruplex restricts the orientation of the guanine bases about the glycosidic bond to anti or syn configuration depending on the parallel/antiparallel nature of the strand. ${ }^{140,141}$

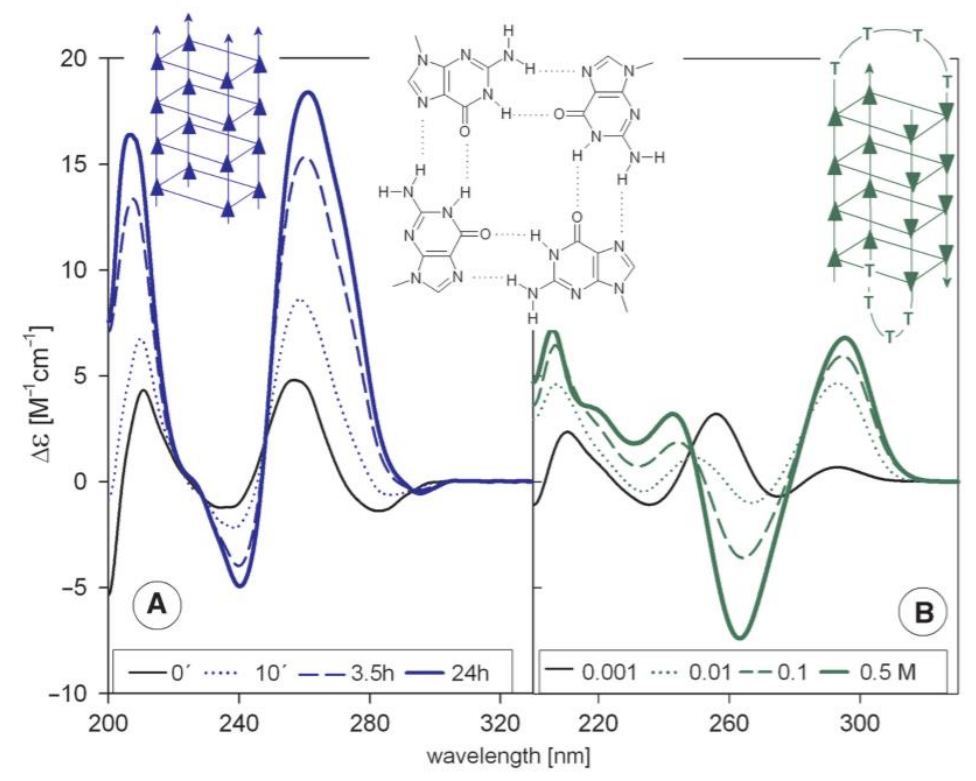

Figure 1. 8: CD spectra of parallel (A) vs anti-parallel (B) G-quadruplex motifs. The orientation of the guanine residues composing the $G$ tetrad, whether syn- or anti-, dictate the nature of the quadruplex and is easily distinguished through their $C D$ spectra by the presence of inverse ellipticities. Used with permission from Oxford Academic. ${ }^{137}$

\subsection{Literature aptamers for monomeric and oligomeric $\alpha$-synuclein}

Despite having known pathological implications in PD, inhibition of $\alpha$-synuclein has not been extensively analyzed with aptamer therapeutics. However, several papers throughout literature have recognized aptamers displaying high binding affinities for both monomeric and oligomeric forms of $\alpha$-synuclein. ${ }^{105,142,143}$

The first aptamer selected for $\alpha$-synuclein was initially published by Tsukakoshi et al. in $2010 .{ }^{142}$ Following the fifth round of SELEX, 14 sequences in total were identified with specific binding for monomeric $\alpha$-synuclein where the sequence exhibiting the highest 
affinity was labelled M5-15. M5-15 was a 66-mer sequence consisting of one centralized and two terminal stem-loops. Truncations performed on M5-15 to determine which conserved regions contributed most to target binding found that all three stem-loops are essential for protein interaction. Dot blotting assay selectivity tests were performed using both monomeric and oligomeric $\alpha$-synuclein where M5-15 was more selective for the oligomeric conformations. Their reasoning was that the oligomeric structure forms a more rigid conformation containing the necessary binding regions on the surface for aptamer recognition. ${ }^{142}$

In 2012 Tsukakoshi et al, ${ }^{105}$ equipped with the knowledge of their M5-15 aptamer, performed a competitive screening selection assay using monomeric, oligomeric, and fibril forms of $\alpha$-synuclein on a randomized oligonucleotide library. Following their selection procedure, eight aptamer sequences were generated exhibiting specific binding for oligomeric $\alpha$-synuclein that could selectively discriminate against both monomeric and fibril forms of $\alpha$-synuclein. Two sequences in particular, T-SO508 and T-SO530, were estimated to have dissociation values $\left(\mathrm{K}_{\mathrm{d}}\right)$ of 68 and $63 \mathrm{nM}$ respectively. It was determined that these sequences displayed intramolecular G-quadruplex characteristics due to their Grich nature that could lead to greater affinity. Unfortunately, the specificity of their sequence was not ideal; when exposed to $\mathrm{A} \beta_{1-40}$, T-SO508 bound with a $\mathrm{K}_{\mathrm{d}}$ value of 25 $\mathrm{nM}$, slightly better than that of oligomeric $\alpha$-synuclein. Their reasoning was that the aptamers can recognize a conserved region within amyloid oligomers and are not limited to the monomeric $\alpha$-synuclein sequence.

The most recent literature published by Zheng et al. in $2018^{143}$ focused on two aptamers selected for monomeric $\alpha$-synuclein that could effectively reduce in vitro $\alpha$ - 
synuclein aggregation and target $\alpha$-synuclein to lysosomal degradation. The two aptamers, F5R1 and F5R2, are 58-mer in length with respective $\mathrm{K}_{d}$ values of $2.40 \mathrm{nM}$ and $3.07 \mathrm{nM}$ exhibited fibril inhibition in aptamer: fibril concentrations as low as 10:1. Delivering F5R1 and F5R2 to primary neuronal cells responsible for overexpressing $\alpha$-synuclein resulted in a reduction of aggregation at aptamer concentrations of $20 \mathrm{nM}$. The presence of F5R1 and F5R2 within these cells lead to reduced $\alpha$-synuclein association with mitochondria, minimizing mitochondrial dysfunction and impairment, oxidative stress, and increasing cellular function.

SELEX was performed by Josh Callahan for the protein target $\alpha$-synuclein using a previously identified literature aptamer, M515, as a template. ${ }^{142,144}$ A depiction of the SELEX process is illustrated in Figure 1.9 where five aptamer sequences, labelled ASYN(1-5), were designed and identified as having a high binding affinity for monomeric $\alpha$-synuclein following five rounds of selection.

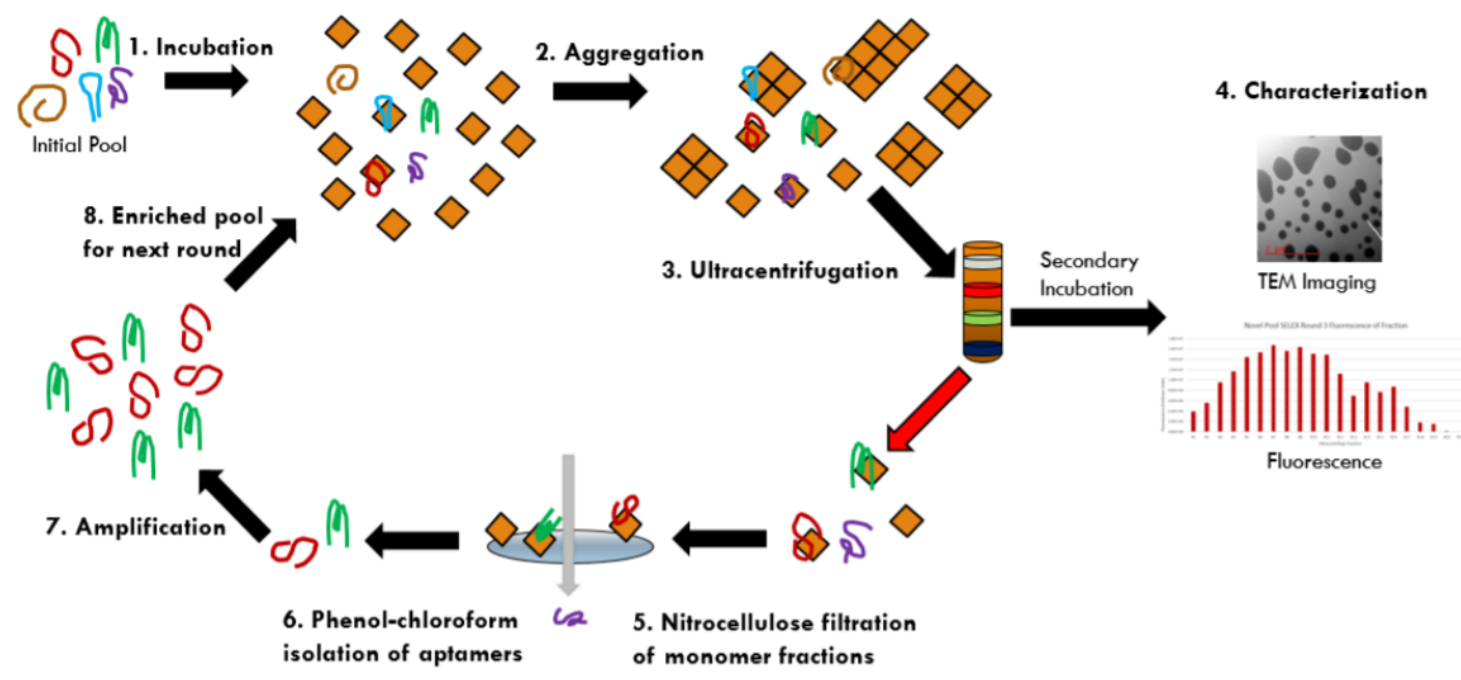

Figure 1. 9: SELEX process used to identify and generate $\operatorname{ASYN(1-5)}$ aptamer sequences for monomeric $\alpha$-synuclein. Reproduced with permission. ${ }^{144}$ 
ASYN2 was deemed to be the most selective aptamer and became the therapeutic aptamer

of interest moving forward. Vernon Hunt ${ }^{145}$ performed conformational and aggregation inhibition analysis on ASYN(1-5) where it was determined that ASYN2 is capable of adopting a G-quadruplex motif when subjected to physiological conditions while showing some degree of aggregation inhibition.

\subsection{Thesis objectives}

The objectives of this thesis were to characterize previously generated $\alpha$-synuclein aptamers from the DeRosa lab, ASYN(1-5), for their binding affinity and selectivity, as well as their ability to inhibit $\alpha$-synuclein aggregation. These data were compared to those of aptamers reported in literature that have been selected against both monomeric and oligomeric $\alpha$-synuclein species. First, preliminary binding affinity, target specificity, and secondary structure analysis of ASYN(1-5) in the presence of monomeric $\alpha$-synuclein will be performed using various optimized techniques. Next, ASYN2 minimer species, A2m(16), were designed and characterized through UV-Vis and CD in an effort to develop a truncated sequence with equivalent structural properties and $\mathrm{K}_{\mathrm{d}}$ as the parent sequence. Finally, both ASYN(1-5) and A2m(1-6) sequences were compared with other aptamers from the literature to determine their ability to inhibit the progression of monomeric $\alpha$ synuclein into oligomeric and fibril aggregates. 


\section{Chapter 2: Experimental}

\subsection{Chemicals and reagents}

Tris(2-carboxyethyl)phosphine (TCEP), 5,5'-dithiobis-2-nitrobenzoic acid (DTNB), dithiothreitol (DTT), potassium ferricyanide $\left(\mathrm{K}_{3}\left[\mathrm{Fe}(\mathrm{CN})_{6}\right]\right)$, potassium ferrocyanide $\left(\mathrm{K} 4\left[\mathrm{Fe}(\mathrm{CN})_{6}\right]\right)$, 6-mercapto-1-hexanol $(\mathrm{MCH})$, tetrachloroauric(III) acid $\left(\mathrm{HauCl}_{4} \cdot 3 \mathrm{H}_{2} \mathrm{O}\right.$ ), trisodium citrate, $20 \%$ poly(diallyldimethyl ammonium chloride) (PDDA), and uranyl acetate were purchased from Sigma-Aldrich (USA). Hydrogen peroxide $\left(35 \%, \mathrm{H}_{2} \mathrm{O}_{2}\right)$ was purchased from Caledon Laboratory Chemicals (Canada). Ammonium hydroxide $\left(28 \%, \mathrm{NH}_{4} \mathrm{OH}\right)$ hydrochloric acid $(\mathrm{HCl})$, sulfuric acid $\left(\mathrm{H}_{2} \mathrm{SO}_{4}\right)$, and nitric acid $\left(\mathrm{HNO}_{3}\right)$ were purchased from Anachemia (Canada). Sodium chloride $(\mathrm{NaCl})$, sodium hydroxide $(\mathrm{NaOH})$, potassium chloride $(\mathrm{KCl})$, potassium phosphate monobasic $\left(\mathrm{KH}_{2} \mathrm{PO}_{4}\right)$, potassium phosphate dibasic $\left(\mathrm{K}_{2} \mathrm{HPO}_{4}\right)$, magnesium chloride $\left(\mathrm{MgCl}_{2}\right)$ were purchased from Bioshop (Canada). $\alpha$-synuclein (monomer) and pre-formed fibril (PFF) sample were purchased from Proteos (USA), $\alpha$-synuclein A53T mutant, $\beta$-synuclein, and $\gamma$-synuclein were purchased from rPeptide (USA). All protein samples were stored at $20^{\circ} \mathrm{C}$. Ac-dC-CE, dmf-dG-CE, dT-CE, and dA-CE phosphoramidites, thiol modifier C6 SS, $1000 \AA$ solid support columns, 2 M TEAA acetonitrile (ACN), cap mix A, cap mix B, oxidizer, deblock, and activator reagents were purchased from Glen Research (USA). Ultra-high purity 5.0 argon, 5.0 nitrogen, and dry ice were purchased from Praxair. All reagents were analytical grade and used without further purification. All solutions were prepared in deionized water $\left(\mathrm{DiH}_{2} \mathrm{O}, 18.0 \mathrm{M} \Omega \cdot \mathrm{cm}\right.$ resistivity) from a Millipore MilliQ system. Various buffers were prepared throughout this chapter. DNA was stored in PBS containing $1 \mathrm{M} \mathrm{NaCl}, 10 \mathrm{mM}$ phosphate, $\mathrm{pH} 7.4$ (BFR-1), DNA disulfide cleavage was 
performed in $10 \mathrm{mM}$ TCEP, $1 \mathrm{M} \mathrm{NaCl}, 10 \mathrm{mM}$ phosphate, $\mathrm{pH} 7.4$ (BFR-2), aptasensormodified electrodes were washed in $10 \mathrm{mM} \mathrm{NaCl}, 5 \mathrm{mM}$ Tris, $\mathrm{pH} 7.4$ (BFR-3), electrochemical measurements were performed in PBS with electrolyte comprising $5 \mathrm{mM}$ $\left[\mathrm{Fe}(\mathrm{CN})_{6}\right]^{3-14-}$ and $0.1 \mathrm{M} \mathrm{KCl}(\mathrm{BFR}-4)$.

\subsection{DNA oligonucleotide synthesis}

Single stranded DNA oligonucleotides were synthesized using phosphoramidite chemistry on a BioAutomation Corporation MerMade 6 DNA Synthesizer (USA) at a 1 $\mu$ mol scale with $1000 \AA$ controlled pore glass (CPG) columns. Using standard manufacturer synthesis conditions, the corresponding sequences were synthesized (Table 2.1). Following DNA synthesis, DNA cleavage was performed by removing the columns from the synthesizer and transferring the dried CPG beads to $1 \mathrm{~mL} 28 \% \mathrm{NH}_{4} \mathrm{OH}$ were they were maintained at $65^{\circ} \mathrm{C}$ for $2 \mathrm{~h}$ or $25^{\circ} \mathrm{C}$ for $24 \mathrm{~h}$. Once cooled to $25^{\circ} \mathrm{C}, 1 \mathrm{~mL}$ of $100 \mathrm{mg} / \mathrm{mL}$ $\mathrm{NaCl}$ was added to the respective DNA solutions. DNA purification was performed using Glen-Pak protocol. 150 mg Glen-Pak DNA purification cartridges and a 12-port vacuum manifold were obtained from Glen Research (USA). In brief, the vacuum pressure was adjusted to $7 \mathrm{mmHg}$ where the purification columns were prepared using $0.5 \mathrm{~mL} \mathrm{ACN}$, followed by $1 \mathrm{~mL} 2 \mathrm{M}$ TEAA. The $2 \mathrm{~mL}$ oligonucleotide-salt mixtures were added to their respective columns where subsequent $2 \times 1 \mathrm{~mL}$ washes of $5 \% \mathrm{ACN}$ in $100 \mathrm{mg} / \mathrm{mL} \mathrm{NaCl}$, 4\% TFA, and $\mathrm{DiH}_{2} \mathrm{O}$ were applied. The washes were discarded prior to the purified DNA's elution with $1 \times 1 \mathrm{~mL} \mathrm{50 \%} \mathrm{ACN}$ in $\mathrm{DiH}_{2} \mathrm{O}$ containing $0.5 \% \mathrm{NH}_{4} \mathrm{OH}$. Eluant was dried overnight on a Savant AES2010 SpeedVac system. The dried, purified DNA was resuspended in BFR-1 and characterized using a Cary Bio 300 UV-Vis Spectrophotometer 
where the $260 \mathrm{~nm}$ peak was analyzed. $1 \mathrm{nmol}$ of the respective DNA samples was sent off for mass spec confirmation at the Mass Spectrometry Centre at McGill University (Appendix B).

Table 2. 1: Synthesized aptamer sequences used for characterization tests and inhibition assays. Colored segments denote the 5'-PBR (red), random region (black), 3'-PBR (blue), 5'-thiol modification (green), and 5'-Cy5 modification (orange). Extinction coefficients and theoretical $T_{m}$ were predicted suing IDT OligoAnalyzer software.

\begin{tabular}{|c|c|c|c|c|}
\hline Aptamer & Sequence $\left(5^{\prime} \rightarrow 3^{\prime}\right)$ & Length & $\begin{array}{l}\text { Extinction Coefficient } \\
\left(\mathrm{L} \mathrm{mol}^{-1} \mathrm{~cm}^{-1}\right)\end{array}$ & $\begin{array}{c}\text { Theoretical } \\
T_{m}\left({ }^{\circ} \mathrm{C}\right) \\
\end{array}$ \\
\hline ASYN1 & $\begin{array}{l}\text { 5' - ATA GTC CCA TCA TTC ATT } \\
\text { GTA TGG TAC GGC GCG GTG } \\
\text { GCG GGT GCG GGG AGA TAT } \\
\text { TAG CAA GTG TCA }\end{array}$ & 66 & 647400 & 72.7 \\
\hline ASYN2 & $\begin{array}{l}\text { 5' - ATA GTC CCA TCA TTC ATT } \\
\text { GTA AGG AAA CGC TAC GGG } \\
\text { GTG GGT ACG GCA AGA TAT } \\
\text { TAG CAA GTG TCA }\end{array}$ & 66 & 662800 & 70.0 \\
\hline ASYN3 & $\begin{array}{l}\text { 5’ }^{\prime} \text { ATA GTC CCA TCA TTC ATT } \\
\text { GTA TGA GAT GGG GTG GTG } \\
\text { ACG TCA GCA TGG AGA TAT } \\
\text { TAG CAA GTG TCA }\end{array}$ & 66 & 662400 & 69.3 \\
\hline ASYN4 & $\begin{array}{l}\text { 5' - ATA GTC CCA TCA TTC ATT } \\
\text { GAA CGG AAT GGC GCG GTG } \\
\text { ACC GGA TAG TGT AGA TAT } \\
\text { TAG CAA GTG TCA }\end{array}$ & 66 & 660700 & 69.9 \\
\hline ASYN5 & $\begin{array}{l}\text { 5' - ATA GTC CCA TCA TTC ATT } \\
\text { GTA TGA TAC AGT GAG GTG } \\
\text { GCA GAT GCA TGC AGA TAT } \\
\text { TAG CAA GTG TCA }\end{array}$ & 66 & 663300 & 68.5 \\
\hline ASYN2R & $\begin{array}{l}5^{\prime} \text { - ACT GTG AAC GAT TAT } \\
\text { AGA ACG GCA TGG GTG GGG } \\
\text { CAT CGC AAA GGA ATG TTA } \\
\text { TTC ACT ACC CTG ATA }\end{array}$ & 66 & 657800 & 70.2 \\
\hline $\mathrm{A} 2 \mathrm{~m} 1$ & $\begin{array}{l}\text { 5' - ATA GTC CCA TCA TTC ATT } \\
\text { GTA AGG AAA CGC TAC GGG } \\
\text { GTG GGT }\end{array}$ & 42 & 414400 & 67.4 \\
\hline $\mathrm{A} 2 \mathrm{~m} 2$ & $\begin{array}{l}5^{\prime} \text { - TAC GGC AAG ATA TTA } \\
\text { GCA AGT GTC A }\end{array}$ & 25 & 257100 & 56.1 \\
\hline $\mathrm{A} 2 \mathrm{~m} 3$ & $\begin{array}{l}\text { 5' - ATA GTC CCA TCA TTC ATT } \text { TTA AGG AAA CGC TAC GGG } \\
\text { GTG GGT ACG GCA }\end{array}$ & 48 & 475500 & 69.6 \\
\hline $\mathrm{A} 2 \mathrm{~m} 4$ & $\begin{array}{l}\text { 5' - GTA AGG AAA CGC TAC } \\
\text { GGG GTG GGT ACG GCA AGA } \\
\text { TAT TAG CAA GTG TCA }\end{array}$ & 48 & 491700 & 68.8 \\
\hline $\mathrm{A} 2 \mathrm{~m} 5$ & 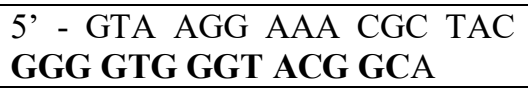 & 30 & 304400 & 67.6 \\
\hline $\mathrm{A} 2 \mathrm{~m} 6$ & 5' - GGG GTG GGT ACG GC & 14 & 136600 & 56.0 \\
\hline
\end{tabular}




\begin{tabular}{|c|c|c|c|c|}
\hline ASYN1-TM & $\begin{array}{l}\text { 5' - C6-S-S-ATA GTC CCA TCA } \\
\text { TTC ATT GTA TGG TAC GGC } \\
\text { GCG GTG GCG GGT GCG GGG } \\
\text { AGA TAT TAG CAA GTG TCA }\end{array}$ & 66 & 647400 & 72.7 \\
\hline ASYN2-TM & 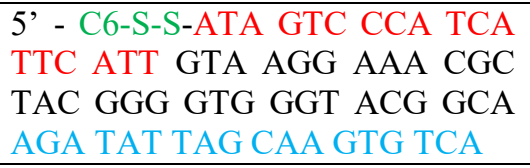 & 66 & 662800 & 70.0 \\
\hline ASYN3-TM & $\begin{array}{l}5^{\prime}-\text { C6-S-S-ATA GTC CCA TCA } \\
\text { TTC ATT GTA TGA GAT GGG } \\
\text { GTG GTG ACG TCA GCA TGG } \\
\text { AGA TAT TAG CAA GTG TCA }\end{array}$ & 66 & 662400 & 69.3 \\
\hline ASYN4-TM & $\begin{array}{l}5^{\prime}-\text { C6-S-S-ATA GTC CCA TCA } \\
\text { TTC ATT GAA CGG AAT GGC } \\
\text { GCG GTG ACC GGA TAG TGT } \\
\text { AGA TAT TAG CAA GTG TCA }\end{array}$ & 66 & 660700 & 69.9 \\
\hline ASYN5-TM & $\begin{array}{l}5^{\prime}-\text { C6-S-S-ATA GTC CCA TCA } \\
\text { TTC ATT GTA TGA TAC AGT } \\
\text { GAG GTG GCA GAT GCA TGC } \\
\text { AGA TAT TAG CAA GTG TCA }\end{array}$ & 66 & 663300 & 68.5 \\
\hline ASYN2-Cy5 & $\begin{array}{l}5^{\prime}-\text { Cy-5-ATA GTC CCA TCA } \\
\text { TTC ATT GTA TGG TAC GGC } \\
\text { GCG GTG GCG GGT GCG GGG } \\
\text { AGA TAT TAG CAA GTG TCA }\end{array}$ & 66 & 647400 & 72.7 \\
\hline M5-15 $5^{142}$ & $\begin{array}{l}\text { ' - ATA GTC CCA TCA TTC ATT } \\
\text { GTA TGG TAC GGC GCG GTG } \\
\text { GCG GGT GCG TGG AGA TAT } \\
\text { TAG CAA GTG TCA }\end{array}$ & 66 & 646000 & 72.2 \\
\hline T-SO508 & $\begin{array}{l}5^{\prime}-\text { GCC TGT GGT GTT GGG } \\
\text { GCG GGT GCG }\end{array}$ & 24 & 220200 & 71.0 \\
\hline T-SO530 $0^{105}$ & $\begin{array}{l}5^{\prime}-\text { GGT GCG GCG GGA CTA } \\
\text { GTG GGT GTG }\end{array}$ & 24 & 232300 & 67.7 \\
\hline F5R1 $1^{143}$ & $\begin{array}{l}5^{\prime}-\text { ATC GAG TGT GTA CGG } \\
\text { GGT CCG GTA GGG TGG CGA } \\
\text { GGT CTT CCT GTC GTA GCA } \\
\text { GGA TCC A }\end{array}$ & 58 & 560900 & 74.0 \\
\hline F5R2 $2^{143}$ & $\begin{array}{l}5^{\prime} \text { - ATC GAG TGG ACG AGT } \\
\text { GCC TCC GGT ACG AGC TGT } \\
\text { CTG ATG GGT TTG CGC GCA } \\
\text { GGA TCC A }\end{array}$ & 58 & 552200 & 74.6 \\
\hline TRA & $\begin{array}{l}\text { '-GAA TTC CGC GTG TGC ACA } \\
\text { CGG TCA CAG TTA GTA TCG } \\
\text { CTA CGT TCT TTG GTA GTC } \\
\text { CGT TCG GGA T }\end{array}$ & 64 & 603000 & 71.3 \\
\hline
\end{tabular}

\subsection{Electrochemical instrumentation}

A CHI660C electrochemical workstation (Austin, Texas) was used for all electrochemical measurements. A three-electrode electrochemical cell configuration was employed comprising an aptamer-modified gold electrode (diameter $1.6 \mathrm{~mm}$ ) as the 
working electrode, an $\mathrm{Ag} / \mathrm{AgCl} 1 \mathrm{M} \mathrm{KCl}$ electrode as the reference electrode, and a platinum wire (diameter $2 \mathrm{~mm}$ ) as the counter electrode. The $15 \mu \mathrm{L}$ electrochemical cell was enclosed within a grounded Faraday cage.

\subsection{Aptasensor fabrication}

Thiol-modified aptamer (DNA-S-S) was suspended and cleaved in $1 \mathrm{~mL}$ BFR-2 for 60 min with constant agitation prior to electrode preparation. The bare gold electrodes were polished for $3 \mathrm{~min}$ in aqueous slurries containing 0.3 and $0.05 \mu \mathrm{m}$ alumina and diamond powders respectively (BASi, USA). The polished working electrode was immersed into freshly prepared piranha solution (3:1 (v/v) $\left.98 \% \mathrm{H}_{2} \mathrm{SO}_{4} / 30 \% \mathrm{H}_{2} \mathrm{O}_{2}\right)$ for 3 min at $25^{\circ} \mathrm{C}$. Electrodes were extensively rinsed with $\mathrm{DiH}_{2} \mathrm{O}$ prior to electrochemical cleaning in $1 \mathrm{M}$ $\mathrm{H}_{2} \mathrm{SO}_{4}$ where potential scanning between $-0.25 \mathrm{~V}$ and $1.6 \mathrm{~V}$ was applied for 50 cycles, or until a reproducible cyclic voltammogram was produced. Freshly cleaved thiolated aptamer (DNA-SH) was heated to $90^{\circ} \mathrm{C}$ for $5 \mathrm{~min}$ and cooled to $25^{\circ} \mathrm{C}$ to allow stable secondary structure prior to electrode immobilization. The gold electrodes were incubated in BFR-1 containing $1 \mu \mathrm{M}$ aptamer for $2 \mathrm{~h}$ at $25^{\circ} \mathrm{C}$. Following the incubation, aptamermodified electrodes (Apt/Au electrode) were rinsed with $\mathrm{DiH}_{2} \mathrm{O}$ before treatment with 1 $\mathrm{mM} \mathrm{MCH}$ for $60 \mathrm{~min}$. The aptasensor (MCH/Apt/Au electrode) was washed in BFR-3 for 10 min with soft stirring to remove unfixed $\mathrm{MCH}$ and Apt before being dried with argon. The prepared aptasensor was stored in BFR-1 at $25^{\circ} \mathrm{C}$ until further use.

\subsection{Ellman's reagent}

To determine the success of the ASYN disulfide cleavage, the Ellman's reaction was applied to quantify free thiol concentration in solution through the reaction of Ellman's 
reagent (DTNB) with the free terminal thiol on the aptamer. In the presence of excess TCEP (10 mM), ASYN-SH was incubated in BFR-2 for $1 \mathrm{~h}$ before being placed in a $3 \mathrm{kDa}$ cutoff desalting microcentrifuge column and rinsed with $\mathrm{diH}_{2} \mathrm{O}$ for four successive washes at 12000 RPM for $20 \mathrm{~min}$. The desalted samples were removed from the desalting columns by inverting the respective columns and placing them at $2000 \mathrm{RPM}$ for $4 \mathrm{~min}$. The cleaved ASYN sample was then exposed to DTNB in a ratio of $1: 1$, where the formation of $\mathrm{TNB}^{2-}$ could be quantified at $412 \mathrm{~nm}$ to determine the availability of free thiol in solution.

\subsection{Electrochemical measurements}

Cyclic voltammetry (CV) analysis was performed using BFR-4 to determine the electroactive surface area of the working electrodes. A variable scan rate between $10-200$ $\mathrm{mV} / \mathrm{s}$ and range of $-0.1 \mathrm{~V}$ to $0.8 \mathrm{~V}$ were applied to the system according to the RandlesSevcik equation. Electrochemical impedance spectroscopy (EIS) measurements were performed in $10 \mathrm{~mL}$ BFR-4. EIS measurements were performed with a frequency range of $0.01-100 \mathrm{kHz}$ and a signal amplitude of $5 \mathrm{mV}$. The aptasensor was immersed in various concentrations of alpha-synuclein monomer for $60 \mathrm{~min}$ at $25^{\circ} \mathrm{C}$ and thoroughly rinsed with BFR-1 to remove unbound target. The changes in impedance were recorded before and after incubation with $\alpha$-synuclein. EIS spectra were displayed as a Nyquist plot $\left(-Z_{\text {im }}\right.$ vs $\mathrm{Z}_{\mathrm{re}}$ ) using $\mathrm{CHI}$ software and fitted with a theoretical curve corresponding to Randles equivalent circuit. Each measurement was repeated four times, and control experiments for $\alpha$-synuclein A53T mutant, $\alpha$-synuclein fibril, $\beta$-synuclein, $\gamma$-synuclein, and thrombin control were carried out in identical conditions. 


\subsection{Aptamer binding constant}

The binding constant $\left(\mathrm{K}_{\mathrm{d}}\right)$ of $\alpha$-synuclein monomer to the fabricated aptasensor was determined by fitting the measured EIS signals as a function of $\alpha$-synuclein monomer concentration using Sigmaplot single binding regression analysis.

\subsection{Gold nanoparticle (AuNP) synthesis}

Glassware used for AuNP synthesis was cleaned by soaking in aqua regia (3:1 (v/v) $\mathrm{HCl} / \mathrm{HNO}_{3}$ ) for 60 min prior to thorough rinsing with $\mathrm{DiH}_{2} \mathrm{O}$. AuNPs were prepared by a sodium citrate reduction of $\mathrm{HAuCl}_{4}$. $\mathrm{HAuCl}_{4}(2 \mathrm{~mL}, 50 \mathrm{mM})$ was added to a $250 \mathrm{~mL}$ Erlenmeyer flask containing $\mathrm{DiH}_{2} \mathrm{O}(98 \mathrm{~mL})$ and brought to a vigorous boil with constant stirring. Upon boiling, trisodium citrate $(10 \mathrm{~mL}, 38.8 \mathrm{mM})$ was added to the solution where a colour change occurred from yellow to dark blue, purple, and wine red. Upon turning red, the solution was boiled for an additional 15 min before being removed from heat and brought to $25^{\circ} \mathrm{C}$. The synthesized AuNPs were stored at $25^{\circ} \mathrm{C}$ and the nanoparticle size was estimated using UV-V is spectroscopy where the maximum absorbance occurred at $525 \mathrm{~nm}$.

\subsubsection{Colorimetric assay}

DNA aptamer $(16 \mu \mathrm{L}, 1 \mu \mathrm{M})$ was reacted with various concentrations of $\alpha$ synuclein monomer and brought to a volume of $85 \mu \mathrm{L}$ with $\mathrm{DiH}_{2} \mathrm{O}$. After 30 min at $25^{\circ} \mathrm{C}$, PDDA $\left(15 \mu \mathrm{L}, 1.89 \times 10^{-4} \mathrm{mM}\right)$ in $\mathrm{DiH}_{2} \mathrm{O}$ was added for $60 \mathrm{~s}$ before addition of AuNP (50 $\mu \mathrm{L}, 11.3 \mathrm{nM})$. The solution was agitated where colour and spectra changes were immediately observed by the naked eye and UV-Vis spectroscopy. The solution was transferred to a $1 \mathrm{~cm}$ micro quartz cuvette and the ratio of peak absorbances at $\mathrm{A}_{650}$ and 
$\mathrm{A}_{525}$ in sample solutions were recorded to determine the aggregation of AuNPs. Photographs of the sample were taken using a Samsung S10 and used without further modification.

\subsection{Circular dichroism (CD)}

A Cary-17 Spectrophotometer Conversion (Olis Inc., USA) was utilized to characterize the structural changes and binding properties of aptamers at $25^{\circ} \mathrm{C}$ in the presence and absence of target. An optical chamber of $160 \mu \mathrm{L}$ was used. The CD spectra range was $200-400 \mathrm{~nm}$, scanning speed of $25 \mathrm{~nm} / \mathrm{min}$, bandwidth of $1 \mathrm{~nm}$, response of 1 $\mathrm{s}$, data interval of $1 \mathrm{~nm}$, and a total of four accumulations. Buffer contributions were subtracted from the spectra. Aptamer $(3 \mathrm{nmol})$ and protein target were added together in a 1:1 ratio and brought to a total volume of $160 \mu \mathrm{L}$ with BFR-1. The mixture was incubated together for $1 \mathrm{~h}$ at $25^{\circ} \mathrm{C}$ prior to continuous $\mathrm{CD}$ measurement.

\subsection{Microscale thermophoresis (MST)}

ASYN2-Cy5 modified aptamer was diluted in BFR-1 $(25 \mu \mathrm{L}, 50 \mu \mathrm{M})$ was frozen at $-80^{\circ} \mathrm{C}$ and shipped to 2 bind (Regensburg, Germany) for microscale thermophoresis (MST) analysis on a Monolith NT.115. BFR-1 (5 mL) as well as fresh $\alpha$-synuclein, A53T

$\alpha$-synuclein mutant, $\beta$-synuclein, and $\gamma$-synuclein were shipped with ASYN2-Cy5 on dry ice and analyzed on arrival. According to 2bind procedure, ASYN2-Cy5 was kept at a constant concentration $(50 \mathrm{nM})$ and titrated with various concentrations of target ligands over a range of $305 \mathrm{pM}$ to $10.0 \mu \mathrm{M}$. The assay buffer contained the initial BFR-1 sample along with $0.1 \%$ Tween 20 surfactant, and subjected to an IR laser beam with $40 \%$ power 
and $60 \%$ LED power parameters. Specific binding affinity was determined over the specified concentration range for the respective target ligands.

\subsection{UV-Vis aptamer melting temperature $\left(T_{m}\right)$ studies}

Respective minimer sequences A2m(1-6) were diluted in BFR-1 (3 $\mu \mathrm{M}, 3 \mathrm{~mL})$ and placed into a quartz UV-vis cuvette with a $1 \mathrm{~mm}$ pathlength. A temperature control unit was used with constant stirring where the aptamer absorbance at $295 \mathrm{~nm}$ was analyzed over a temperature gradient of $25-85^{\circ} \mathrm{C}$ with a temperature ramp rate of $2^{\circ} \mathrm{C} / \mathrm{min}$ and hold times of $5 \mathrm{~min}$ once the $\min / \max$ temperature was reached. Two increasing and decreasing temperature cycles were performed for each aptamer where absorbance values were recorded at every $1^{\circ} \mathrm{C}$ interval.

\subsection{Preparation of $\alpha$-synuclein pre-formed fibrils (PFFs)}

The preparation of $\alpha$-synuclein PFFs from monomeric $\alpha$-synuclein was performed according to the protocol developed on the Michael J. Fox Foundation website. To summarize, the purified recombinant $\alpha$-synuclein protein was dissolved in $200 \mu \mathrm{L}$ of BFR1 to obtain a final concentration of $10 \mathrm{mg} / \mathrm{mL} .20 \mu \mathrm{L}$ of PFF stock was placed into a 1.5 $\mathrm{mL}$ microcentrifuge tube where various concentrations of aptamer were added to the PFF stock and diluted to a final volume of $25 \mu \mathrm{L}$ using BFR-1. The respective samples were

placed under argon and wrapped in aluminum foil prior to being subjected to a 7-day incubation period at $37^{\circ} \mathrm{C}$ and $200 \mathrm{RPM}$. Following the 7-day incubation period, PFF samples were removed and placed onto TEM grids for fibril inhibition imaging. 


\subsection{Transmission electron microscopy (TEM)}

400-mesh copper grids coated in carbon film were used for TEM imaging with a FEI Tecnai F20 FETEM. $10 \mathrm{uL}$ of $\alpha$-synuclein PFF sample were placed on the copper TEM grid for 5 min prior to excess removal. The grids were stained with $2 \%$ uranyl acetate for 2 min prior to its removal. AuNP colorimetric studies were performed using 200-mesh copper grids where the respective AuNP sample was place on the grid for 10 mins prior to excess fluid removal. They were imaged as is in the absence of stain. 


\section{Chapter 3: Results and Discussion}

\subsection{Statement of contribution}

Aptamer characterization methodology was discussed and designed by Spencer Boisjoli and Dr. Maria DeRosa. Electrochemical impedance spectroscopy (EIS) was performed and analyzed by Spencer Boisjoli and Dr. Daniela Márquez. Circular dichroism and gold nanoparticle colorimetric assays were performed and analyzed by Spencer Boisjoli and Dr. Maria DeRosa with the assistance of Madison Ferguson, Anna Koudrina, Dr. Ranganathan Velu, Dr. Sathya Srinivasan, and Gabriel Paul. MST analysis was performed by Dr. Maximilian Plach of 2 bind $\mathrm{GmbH}$ and analyzed by Spencer Boisjoli and Dr. Maria DeRosa. ASYN2 minimers were designed and synthesized by Spencer Boisjoli, Gabriel Paul, and Maria DeRosa. Melting temperature studies were performed and analyzed by Spencer Boisjoli and Gabriel Paul. Pre-formed fibril inhibition studies were performed and analyzed by Spencer Boisjoli and Dr. Maria DeRosa. TEM analysis was performed by Spencer Boisjoli and Dr. Jianqun Wang. 


\subsection{Development and characterization of the electrochemical aptasensor}

\subsubsection{Solid phase DNA synthesis by the phosphoramidite method}

The ability to chemically synthesize oligonucleotides, including aptamers, comprises multiple decades of research to the eventual development of the solid phase phosphoramidite approach currently used today. The applicability and automation of phosphoramidite chemistry made oligonucleotide research more feasible for the construction of aptamer sequences. The synthesis cycle is a stepwise addition of four stages: deblocking/detritylation, activation/coupling, capping, and oxidation; proceeding in the $3^{\prime}$ to 5 ' direction opposite to that of DNA polymerase. A solid support column containing 500 or $1000 \AA$ controlled-pore glass (CPG) beads where the 3 '-end of a phosphoramidite is tethered to the bead acts as the starting point for DNA elongation. A phosphoramidite is composed of a respective DNA base (A, C, G, T) with a 4,4'dimethoxytrityl (DMT) protecting group on the 5'-primary hydroxyl of the ribose sugar, and phosphite triester containing $\beta$-cyanoethyl and diisopropylamine (DIPA) protecting groups on the phosphite oxygen atoms. Monomeric nucleosides A, C, and G contain exocyclic amino moieties and require additional protecting groups in the form of benzoyl (Bz), acyl, and dimethylformadine (DmF) respectively. ${ }^{146-149}$

The deprotection stage begins through the addition of dichloroacetic acid (DCA) in an inert solvent, dichloromethane (DCM), that cleaves the acid-labile 5'-DMT group from the terminal nucleoside residue, revealing the reactive nucleophilic 5'-OH. Removal of the DMT group produces an orange colour, indicating the availability of the 5 '-OH for oligonucleotide polymerization. 
The next stage commences through the addition of a phosphoramidite monomer, containing the next base in the DNA sequence, to the free 5'-OH of the terminal nucleoside. An excess of phosphoramidite monomer along with an activator, primarily a weakly acidic tetrazole derivative, is added to the CPG-bound oligonucleotide where the DIPA protecting group is protonated and converted to a good leaving group. Loss of DIPA exposes a nucleophilic hydroxyl group of the incoming phosphoramidite, allowing a new phosphorus-oxygen bond to form with the 5'-OH group of the terminal nucleoside and connect the nucleoside residues via phosphite triester linkage.

Due to the minute concentration of phosphoramidite that did not undergo coupling reactions, a capping stage must be performed to minimize unwanted by-products through acetylating the unreacted $5^{\prime}-\mathrm{OH}$. This stage is performed through the addition of acetic anhydride and catalyst $N$-methylimidazole (NMI) dissolved in a solution of tetrahydrofuran (THF) and pyridine. The acetic anhydride and NMI form an intermediate structure that the exposed nucleophilic 5'-OH group can attack, yielding a capped and acetylated 5'-OH. The presence of acidic conditions may lead to detritylation of the recently coupled phosphoramidite and subsequent acetylation. Minor concentrations of pyridine retain basic $\mathrm{pH}$ conditions that favor the capping reaction to take place and prepare the oligonucleotide sequence for the final oxidation stage.

While the foundation of the natural DNA backbone is a phosphotriester bond and following stage 3 of the synthesis, the resulting oligonucleotide sequence is built on an unnatural phosphite triester bond. It is known that $\mathrm{P}(\mathrm{III})$ is much more reactive than $\mathrm{P}(\mathrm{V})$ and must be oxidized prior to the next cycle. Phosphite triester oxidation is performed using iodine dissolved in a solution containing THF, water, and pyridine. The phosphite is 
oxidized with iodine prior to its displacement with a water molecule. At this stage, the $\mathrm{P}(\mathrm{V})$ backbone is stable and ready to proceed to the next addition cycle. Once the synthesis is completed, purification of the DNA strand is performed after a cleavage step where the beads are subjected to a basic environment to remove the immobilized sequence. Ammonium hydroxide $\left(\mathrm{NH}_{4} \mathrm{OH}\right)$ provides the basic conditions necessary to remove the 5'DMT, $\beta$-cyanoethyl, and heterocyclic nucleoside protecting groups, establishing a complete aptamer sequence. ${ }^{146-149}$

\subsubsection{Aptamer sequences and modifications}

Five 66-mer DNA aptamers, labelled ASYN(1-5), were previously selected for the specific binding of the monomeric protein $\alpha$-synuclein. ${ }^{145}$ Based on the previously discovered M5-15 aptamer capable of binding monomeric $\alpha$-synuclein ${ }^{142}$, the sequence structure of ASYN(1-5) was similar with 3'- and 5'- 18-mer primer binding regions flanking a central 30-mer randomized region. Sequence structures of ASYN(1-5) were generated using RNAstructure software ${ }^{150}$ where the most stable secondary structures were analyzed for their theoretical melting temperature and potential regions responsible for target binding. As illustrated in Figure 3.1, ASYN(1-5) are predicted to display unique secondary structures of various stem-loop conformations.

A second online software, QGRS, ${ }^{151}$ analyzed the respective sequences for the presence of a G-quadruplex, where it was found that ASYN2 possessed a G-quadruplex within its centralized region. A G-quadruplex is an association of four guanine bases (tetrad) connected through Hoogsteen hydrogen bonds to form a square planar complex. 
From Figure 3.2, a total of eight hydrogen bonds occur within this secondary conformation between the $\mathrm{N} 1-\mathrm{H}$ and $\mathrm{O} 6$ positions, and the $\mathrm{N} 2-\mathrm{H}$ and N7 positions respectively. ${ }^{152}$

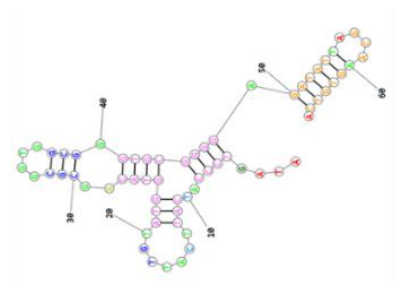

ASYN1

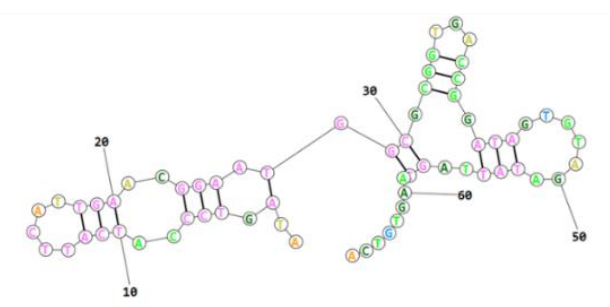

ASYN4

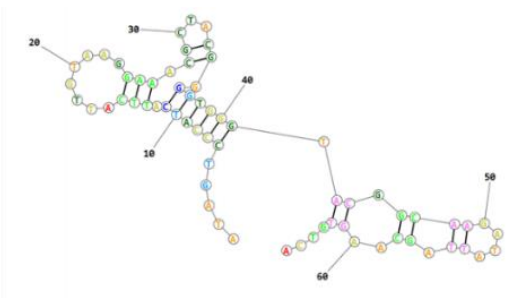

ASYN2

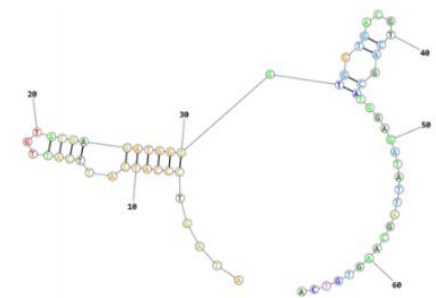

ASYN3

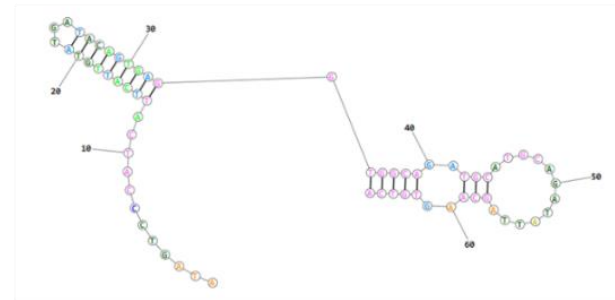

ASYN5

Figure 3. 1: (A) Predicted secondary structures visualizing the free energy conformations of ASYN(15) sequences in accordance with RNAstructure. (B) The colored bases correspond to their respective base pairing probabilities while the free energy of the respective aptamers is determined in terms of $\mathrm{kJ} \mathrm{mol}^{-1}$.

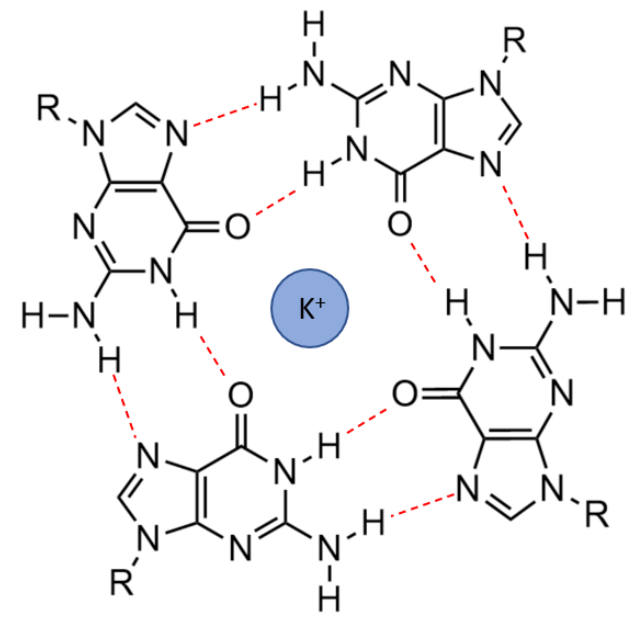

Figure 3. 2: Representation of a G-tetrad composed of four guanine residues. A G-quadruplex motif is typically comprised of several tetrads where Hoogsteen bonding interactions are illustrated using a dashed line. 
Within a G-quadruplex, several stacked guanine tetrads can result from intramolecular or intermolecular strand interactions with the non-guanine nucleotides assuming single stranded loops in between. Due to base stacking stabilizing properties, it is common to observe a G-quadruplex comprised of multiple tetrads where the sequence will have several guanine rich segments. Depending on strand directionality and orientation, the tetrad can be comprised of guanosine stems adopting syn or anti glycosidic bond angles and leading to various G-quadruplex topologies such as parallel, antiparallel, or a hybrid of the two. ${ }^{153,154}$ Alkali metal cations play a significant role in G-quadruplex structural stability with sodium and potassium ions commonly used throughout literature. Guanine carbonyl groups are angled towards the center of the $G$ tetrad, forming a negatively charged inner channel the metal cations can interact with. Potassium ions are more ideal for quadruplex stability due to their larger ionic radius. The smaller sodium ions stabilize the tetrad within the plane, whereas the larger potassium ion can situate itself in between two tetrads. G-quadruplexes have been investigated for their biological relevance in microorganisms ${ }^{155}$ and have been exploited in aptamers for their potential in forming anticancer drug complexes. ${ }^{154}$ Presence of a G-quadruplex is theorized as an integral part of this aptamers binding to monomeric $\alpha$-synuclein as this aptamer displayed the highest target binding affinity during its selection. Alternative secondary structures will also be considered for binding to monomeric $\alpha$-synuclein. Several aptasensors have been developed utilizing the conformational change of an aptamers G-quadruplex when exposed to its target. ${ }^{156,157}$

Figure 3.3 displays the G-score for the individual aptamers according to analysis performed using QGRS software alongside sequences for $\operatorname{ASYN}(1-5)$ with the G- 
quadruplex motif highlighted in red and guanine residues forming the G-tetrads highlighted in yellow.

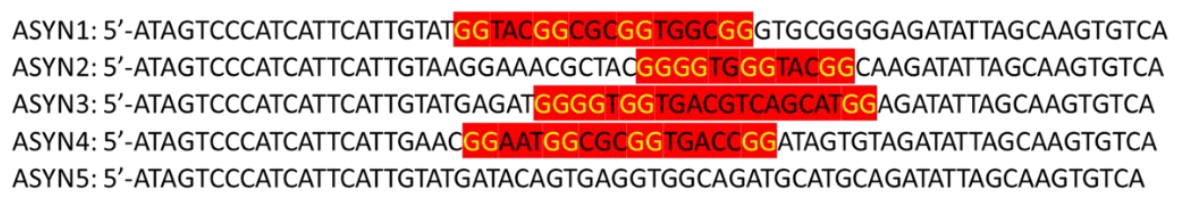

\begin{tabular}{|c|c|}
\hline Sequence & G-Score \\
\hline ASYN1 & 20 \\
\hline ASYN2 & 18 \\
\hline ASYN3 & 9 \\
\hline ASYN4 & 19 \\
\hline ASYN5 & 0 \\
\hline
\end{tabular}

Figure 3. 3: (A) ASYN(1-5) sequence comparison with bases involved in G-quadruplex conformation highlighted in yellow with specific guanine tetrad units highlighted in red. (B) The respective G-score, determined using QGRS software, for $\operatorname{ASYN(1-5)}$ indicates that only $\operatorname{ASYN(1-4)}$ are capable of adopting the desired secondary structure.

With the exception of ASYN5, the remaining ASYN(1-4) sequences are capable of folding into a G-quadruplex conformation to varying degrees: ASYN1 $>$ ASYN4 $>$ ASYN2 $>$ ASYN3 $>>>$ ASYN5. What is interesting is the fact that ASYN2 was determined to possess the highest affinity for monomeric $\alpha$-synuclein during its SELEX generation despite having a lower G-score compared to ASYN1 and ASYN4. Potential reasoning for this behaviour is the positioning and length of the G-quadruplex domain within the randomized region of the ASYN sequences. The G-quadruplex length in ASYN2 spans 13 bases, whereas ASYN1 and ASYN4 span 18 and 19 bases respectively. From Figure 3.3 a noticeable shift also occurs in G-quadruplex positioning where it resides just prior to the 3' PBR in ASYN2 unlike the more centralized location visible in ASYN1 and ASYN4. The reduced size and shift of the ASYN2 motif could potentially decrease steric restraints experienced by the 5'-PBR, allowing additional freedom and long-range interactions to occur within the aptamer sequence and during target complexation.

For electrochemical aptasensor development, the 5'-end of the respective sequences was functionalized with a thiol modifier consisting of a mercapto group and hexane spacer $\left(5^{\prime}-\left(\mathrm{CH}_{2}\right)_{6}-\mathrm{SH}\right)$. Thiolated DNA have been shown to form a self-assembled 
monolayer (SAM) when exposed to a gold surface due to the rapid chemisorption between the thiol group and the reduced gold surface. ${ }^{158}$ The hexane spacer accompanying the thiol decreases steric hindrance between the surface-anchored aptamer and the gold surface, while increasing its freedom and mobility when exposed to an ionic medium or a specific binding target.

\subsubsection{Electrochemical cell setup and pretreatment}

The electrochemical setup consisted of a three-electrode system comprised of a working electrode, a counter electrode, and a reference electrode. The working electrode (WE) is the most important component of an electrochemical cell and is where all reactions of interest occurred. Potential is applied to the WE as a function of the reference electrode to induce the adsorption/desorption of the electroactive species to the electrode surface. ${ }^{159}$ Several working electrodes have been used throughout literature, with the most common being platinum, ${ }^{71}$ carbon, ${ }^{160}$ and gold. ${ }^{71}$ A gold working electrode (AuE) acted as the aptasensor foundation as it possessed several attractive properties over platinum and carbon electrodes that make them ideal for aptasensor development; primarily being the ease of aptamer immobilization through the formation of SAMs. A standard $\mathrm{Ag} / \mathrm{AgCl}$ electrode was used as the reference electrode (RE) having a negligible current and stable equilibrium potential, is used in comparison to other electrodes within the electrochemical circuit. ${ }^{159}$ The $\mathrm{Ag} / \mathrm{AgCl} \mathrm{RE}$ was filled with $2 \mathrm{M} \mathrm{KCl}$ to minimize the junction potential between the porous membrane of the electrode and the electrolyte, containing $0.1 \mathrm{M} \mathrm{KCl}$. A platinum wire acted as the counter electrode (CE). Within the electrochemical cell, its role was to complete the circuit with the WE by allowing current to flow in the event of electrolytic 
reduction or oxidation at the WE surface. The CE used contained a larger surface area (3.14 $\left.\mathrm{mm}^{2}\right)$ than the WE $\left(2.01 \mathrm{~mm}^{2}\right)$ to minimize interference with the reactions occurring at the WE. The platinum wire is advantageous as it is an inert material and will not contribute to the behavior of the working electrode.

Various electrode pre-treatment steps were performed on the gold electrode to ensure that surface impurities were removed and that the electrochemical behaviour on the electrode surface yielded accurate and reproducible results. These pre-treatment steps occurred immediately before use and consisted of mechanical, chemical, and electrochemical cleaning. Aqueous slurries of alumina $\left(\mathrm{Al}_{2} \mathrm{O}_{3}\right)$ and diamond were placed on respective abrasive micro cloths, where AuEs were polished for a designated period of time. This step aids in recovering the AuE surface as extended use and atmospheric exposure of the surface can lead to its gradual oxidation and a response reduction in the presence of an electrolyte. ${ }^{161}$ A chemical cleaning process was employed second where the AuE was submerged in a 3:1 mixture of concentrated $\mathrm{H}_{2} \mathrm{SO}_{4}: 30 \% \mathrm{H}_{2} \mathrm{O}_{2}$, deemed Piranha solution. Piranha solution is commonly used in electrode cleaning as it is an incredibly strong oxidizer capable of removing organic matter from the AuE surface. ${ }^{162}$ It works primarily through a dehydration process that removes hydrogen and hydroxyl groups as water from organic matter, while the formation of oxygen radical species interacts with residual carbon atoms at the electrode surface.

The final pre-treatment stage is the electrochemical cleaning of the AuE surface in $1 \mathrm{M} \mathrm{H}_{2} \mathrm{SO}_{4}$ using cyclic voltammetry (CV). Represented in Figure 3.4, the working electrode potential is ramped linearly across a specified potential range and plotted as the current exchange between the working and counter electrodes vs the potential applied 
between the working and reference electrodes. Once the maximum potential has been reached, the potential inverts, forming the characteristic triangular waveform. Cyclic voltammograms of an unclean $\mathrm{AuE}$ (curve a) vs a clean $\mathrm{AuE}$ (curve c) where an apparent shift can be observed in both cathodic and anodic current peaks after various consecutive electrochemical sweeps to clean the surface efficiently and reproducibly. An AuE is considered to be clean when the resulting voltammogram is smooth and reproducible as this indicates the surface is stable and homogenous. ${ }^{71,159}$

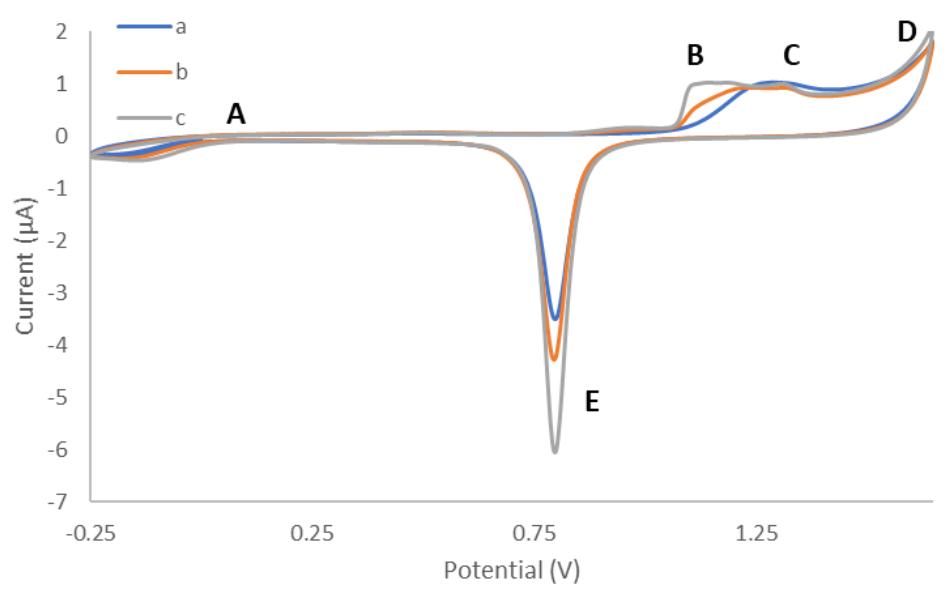

Figure 3. 4: Cyclic voltammogram illustrating the cleaning process of the gold working electrode (AuE) in $1 \mathrm{M} \mathrm{H}_{2} \mathrm{SO}_{4}$ at various stages of electrochemical activation: the initial gold suface (curve a), the gold surface after 10 cycles (curve b), the gold surface after 40 cycles (curve c). Peak A corresponds to the double layer capacitance at the $\mathrm{AuE}-\mathrm{H}_{2} \mathrm{SO}_{4}$ interface, peaks B-E correspond to the various redox reactions that are occurring at the gold interface within the oxide formation region of the $\mathrm{CV}$.

The voltammograms can be isolated into two respective regions to explain the surface processes that are occurring: the double layer region $(-0.2-0.6 \mathrm{~V})$ at the electrodeelectrolyte interface containing the redox peak $\mathrm{A}$, and the oxide formation region $(0.6-$ $1.35 \mathrm{~V})$ on the gold surface containing the redox peaks B, C, D, and E. ${ }^{163-166}$ The surface reactions are illustrated in the following equations: ${ }^{167,168}$ 


$$
\begin{aligned}
& \mathrm{Au}(\mathrm{OH})+2 \mathrm{H}_{2} \mathrm{O}-2 e^{-} \rightarrow \mathrm{Au}(\mathrm{OH})_{3}+2 \mathrm{H}^{+} \\
& 2 \mathrm{H}_{2} \mathrm{O}-4 e^{-} \rightarrow \mathrm{O}_{2}+4 \mathrm{H}^{+} \\
& \mathrm{Au}(\mathrm{OH})+e^{-} \rightarrow \mathrm{Au}+\mathrm{OH}^{-}
\end{aligned}
$$

The increase in anodic current at oxidation peak B is due to the gradual formation of a hydroxyl monolayer on the gold electrode surface while oxidation peaks $\mathrm{C}(1.14 \mathrm{~V})$ and $\mathrm{D}(1.31 \mathrm{~V})$ correspond to equations (3.1) and (3.2) respectively. In this region, oxide formation occurs along the gold interface as adsorbed surface anions are displaced by hydroxyl groups. ${ }^{164}$ One study suggests that peak $\mathrm{C}$ is due to the deposition of $\mathrm{OH}^{-}$between adsorbed surface anions, while peak $\mathrm{D}$ is the simultaneous result of $\mathrm{OH}^{-}$deposition and anion desorption coupled with the formation of $\mathrm{O}_{2} .{ }^{165}$ When the system potential was reversed a single reduction peak, E $(0.81 \mathrm{~V})$, was observed, corresponding to equation 3.3. It has been suggested that the concentration of $\mathrm{H}_{2} \mathrm{SO}_{4}$ used directly impacts the size of the redox peaks. $^{167}$

Electrochemical impedance spectroscopy (EIS) was used as an efficient method of characterizing the stepwise fabrication of the electrochemical AuE aptasensor in the presence of ferri/ferrocyanide redox agents $\left(\left[\mathrm{Fe}(\mathrm{CN})_{6}\right]^{3-/ 4-}\right)$ and is represented in Figure 3.5A. The redox markers $\left[\mathrm{Fe}(\mathrm{CN})_{6}\right]^{3-/ 4-}$ are commonly used throughout literature to examine the charge transfer kinetics that are occurring on the surface of an AuE. Equation 3.4 illustrates the single electron transfer that occurs during ferri/ferrocyanides redox process:

$$
\mathrm{Fe}^{(\mathrm{III})}(\mathrm{CN})_{6}^{3-}+e^{-} \rightleftharpoons \mathrm{Fe}^{(\mathrm{II})}(\mathrm{CN})_{6}{ }^{4-} \quad\left(\mathrm{E}^{\mathrm{o}}=0.356 \mathrm{~V}\right)
$$

It has been described previously that the $\left[\mathrm{Fe}(\mathrm{CN})_{6}\right]^{3-/ 4-}$ redox process is a negatively charged inner-sphere reaction whose electron-transfer kinetics are greatly dependent on 
both the surface chemistry of the modified electrode and the presence of cations in the electrolyte solution. ${ }^{160}$ The electrochemical behavior of the $\left[\mathrm{Fe}(\mathrm{CN})_{6}\right]^{3-14-}$ probe is attributable to the electrostatic interactions experienced with the modified electrode surface where the positive surface charge attracts the negatively charged $\left[\mathrm{Fe}(\mathrm{CN})_{6}\right]^{3-14}$, significantly increasing the rate of electron transfer. ${ }^{169}$ The presence of a supporting electrolyte also impacts the electron transfer kinetics by associating with the electroactive species through ion-pairing, increasing the conductivity of the electrolyte, and maintaining a constant ionic strength. ${ }^{170} \mathrm{KCl}$ acts as the supporting electrolyte in this reaction, having a larger ionic strength then $\left[\mathrm{Fe}(\mathrm{CN})_{6}\right]^{3-/ 4-}$ while remaining inert to the electrochemical reactions at the electrode surface. A general trend of cation ion pairing effects can be observed with increasing cation size: $\mathrm{Li}^{+}<\mathrm{Na}^{+}<\mathrm{K}^{+}<\mathrm{Rb}^{+}<\mathrm{Cs}^{+}$. As cation size increases, its hydration sphere decreases. ${ }^{171}$ Reduced surface solvation would allow the cation to interact with the electroactive species through direct or indirect mechanisms. Electrons would readily transfer from the $\left[\mathrm{Fe}(\mathrm{CN})_{6}\right]^{3-4-}$ probe to the vacant electron orbitals within the cation, increasing the rate of electron transfer that is occurring. ${ }^{171}$

Aptasensor fabrication begins with a clean AuE surface being immobilized with thiolated ssDNA for a period of time. The presence of the mercapto group aids in aptamer orientation within the SAM. The AuE surface was then passivated with $\mathrm{MCH}$ to both prevent non-specific binding of the aptamer to the gold surface, while also coating the surface with an additional substrate to prevent charge transfer from occurring between the electrolyte and the AuE. MCH was selected as it is water soluble and identical length to the thiol-spacer on the DNA. Once fabricated, the MCH/ASYN/AuE forms the basis of the aptasensor prior to target incubation with monomeric $\alpha$-synuclein. 


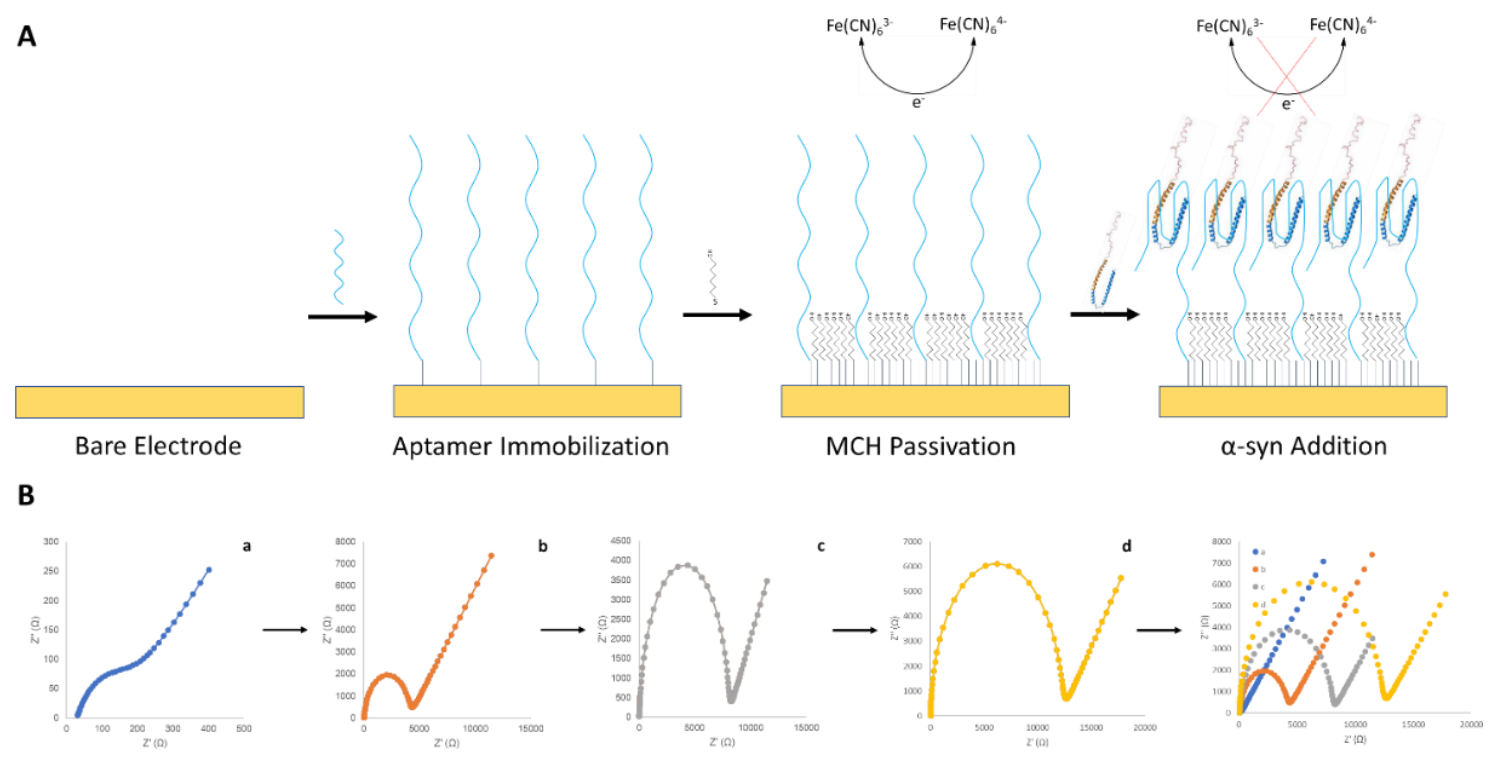

Figure 3. 5: (A) Schematic illustration of electrochemical aptasensor development and mechanism. Physical adsorption of thiolated ssDNA and 6-mercaptohexanol (MCH) occurred on a gold electrode surface in the presence of an electrolyte consisting of $5 \mathrm{mM}\left[\mathrm{Fe}(\mathrm{CN})_{6}\right]^{3-/ 4-}$ and $0.1 \mathrm{M} \mathrm{KCl} \mathrm{in} \mathrm{BFR-1.} \mathrm{B)}$ EIS spectra of the aptasensor at varying stages of the fabrication process lead to an observed increase in charge transfer resistance: (a) bare Au electrode, (b) Aptamer/Au electrode, (c) MCH/Aptamer/Au electrode, (d) Target/MCH/Aptamer/Au electrode. ${ }^{62}$

When performing EIS, the corresponding results are displayed within a Nyquist plot as indicated in Figure 3.5B. A Nyquist plot displays the real $\left(Z^{\prime}\right.$, or $\left.Z_{r e}\right)$ and imaginary components (Z', or $\left.Z_{\mathrm{im}}\right)$ of a systems impedance in the presence of an ionic medium or electrolyte. With a constant potential, the frequency is scanned across a specified range where high-frequencies are representative of the charge transfer surface processes across the electrode double layer, and low-frequencies are representative of electrolyte diffusion processes into the bulk solution. The semicircle and linear portion of the Nyquist plot correspond to high- and low-frequencies respectively. The Nyquist plot is fit to an electrochemical equivalent circuit, referred to as Randle-modified circuit, and can be seen in Figure 3.6 based on the Faradaic charge transfer between the $\left[\mathrm{Fe}(\mathrm{CN})_{6}\right]^{3-/ 4-}$ redox probe and AuE surface. ${ }^{172}$ 


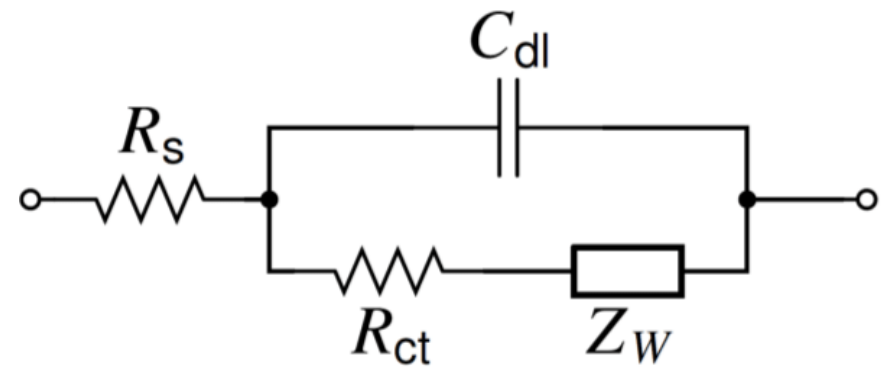

Figure 3. 6: Representation of the Randles electrochemical equivalent circuit. $R_{s}$ represents the electrolyte resistance, $C_{\mathrm{dl}}$ the double layer capacitance at the electrode-electrolyte interface, $\mathbf{R}_{\mathrm{ct}}$ the Faradaic charge transfer resistance between the redox probe and the aptasensor, $Z_{w}$ is Warburg coefficient representing the diffusion of charge from the gold interface to the bulk solution.

The circuit is composed of several parts being the electrolyte resistance $\left(R_{s}\right)$, the double layer capacitance $\left(\mathrm{C}_{\mathrm{dl}}\right)$, the electron charge-transfer resistance $\left(\mathrm{R}_{\mathrm{ct}}\right)$, and the Warburg impedance $\left(\mathrm{Z}_{\mathrm{w}}\right)$. The $\mathrm{R}_{\mathrm{ct}}$ value, being the diffusion of ions across the electrode interface from the electrolyte, can be quantified by the semicircle diameter of the Nyquist plot when fitted to the electrochemical circuit. An increase in the semicircle's diameter represents an increase in charge transfer resistance across the electrode interface and can be observed. As the aptasensor is developed, further addition of substrate to the surface impedes electron transfer across the interface.

The bare AuE displays a very small semicircle due to the ease of electron transfer to the surface where the linear portion indicates a diffusion limiting step $\left(\mathrm{R}_{\mathrm{ct}}=1.6 \times 10^{2} \pm\right.$ $8.6 \Omega$, curve a). When the ASYN aptamer is anchored to the surface, a significant increase in the $\mathrm{R}_{\mathrm{ct}}$ is observed ( $\mathrm{R}_{\mathrm{ct}}=4.8 \times 10^{3} \pm 2.5 \times 10^{2} \Omega$, curve $\mathrm{b}$ ). This increase is a direct result of steric hindrance along the electrode surface that is actively impeding the transfer of electrons from the electrolyte. The presence of DNA not only acts as a poor conductor of electrons across the interface, but the negatively charged phosphate backbone within DNA also electrostatically repels the $\left[\mathrm{Fe}(\mathrm{CN})_{6}\right]^{3-/ 4}$. Surface passivation with $\mathrm{MCH}$ removes non- 
specifically adsorbed DNA, allowing it to adopt a more uniform conformation along the surface. This increases the concentration of $\mathrm{MCH}$ that can interact with the surface, resulting in an increase in impedance $\left(\mathrm{R}_{\mathrm{ct}}=8.8 \times 10^{3} \pm 5.1 \times 10^{2} \Omega\right.$, curve c). Exposure of the aptasensor to target induces a conformational change of the surface-immobilized aptamer as it forms an aptamer-target complex. The aptamer-target complex insulates the electrode surface, contributing to a further increase in charge-transfer resistance. $\left(\mathrm{R}_{\mathrm{ct}}=1.2\right.$ x $10^{4} \pm 6.9 \times 10^{2} \Omega$, curve d).

Cyclic voltammograms were obtained to verify the addition of the various components within aptasensor development (Figure 3.7). The initial peak current of the AuE was $44 \mu \mathrm{A}$ (curve a). After surface immobilization with thiolated-aptamer and $\mathrm{MCH}$, an observable shift and decrease in peak current to $41 \mu \mathrm{A}$ (curve b) and $34 \mu \mathrm{A}$ (curve c) respectively occurred. This agrees with previous impedance data, indicating that a SAM had formed along the electrode surface and hindered the transfer of electrons. Finally, interaction of the aptasensor with monomeric $\alpha$-synuclein forms an aptamer-target complex that further interferes with charge transfer across the electrode-electrolyte interface by reducing the peak current to $29 \mu \mathrm{A}$ (curve d). It is apparent that the magnitude of charge transfer between the redox probe and the electrode surface has been diminished by the presence of electrostatic repulsion on the anions ability to interact with the surface. ${ }^{173}$ The irreversible nature of the current peaks results from the inability of redox anions to effectively interact with the surface due to the impedance imparted on electrodeelectrolyte charge transfer by the developing aptasensor. The EIS and CV data suggest that the aptasensor was successfully fabricated and can be effectively used to determine the $\mathrm{K}_{\mathrm{d}}$ of the respective ASYN aptamers when subjected to monomeric $\alpha$-synuclein. 


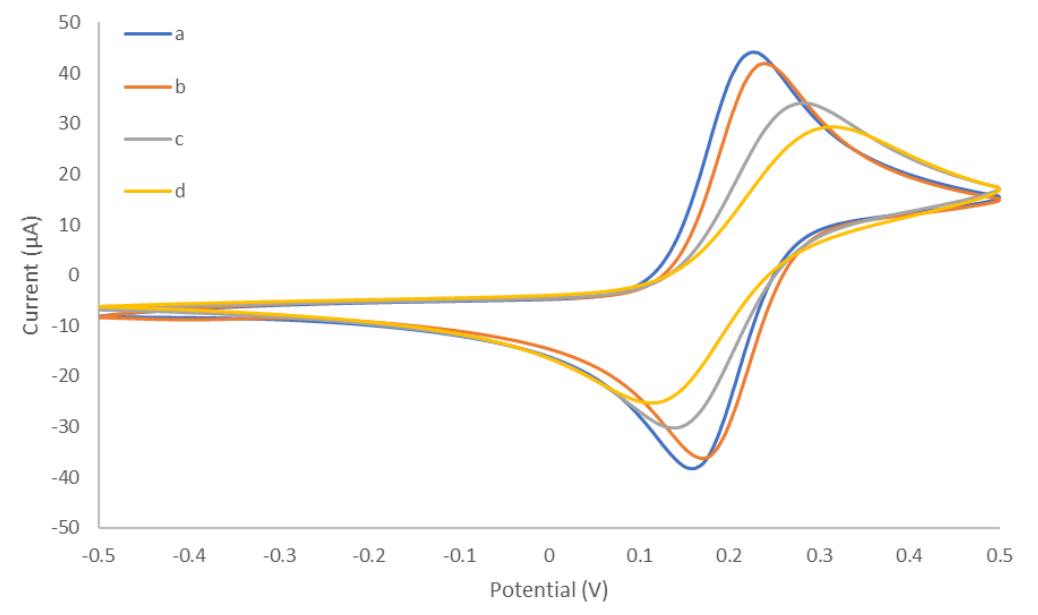

Figure 3. 7: Cyclic voltammogram depicting aptasensor fabrication in the presence of BFR-1 containing $5 \mathrm{mM}\left[\mathrm{Fe}(\mathrm{CN})_{6}\right]^{3-4-}$ and $0.1 \mathrm{M} \mathrm{KCl}$. Voltammogram was performed over a potential range of $-0.5 \mathrm{~V}$ to $0.2 \mathrm{~V}$ at a scan rate of $200 \mathrm{mV}$. Respective aptasensor components are defined as: AuE/Buffer (curve a), Apt/AuE/Buffer (curve b), MCH/Apt/AuE/Buffer (curve c), Target/MCH/Apt/AuE/Buffer (curve d).

\subsubsection{Optimization of aptasensor conditions}

Cyclic voltammetry was performed to determine the available electroactive surface area present on the AuEs in the presence of $5 \mathrm{mM}\left[\mathrm{Fe}(\mathrm{CN})_{6}\right]^{3-/ 4-}$ as the test solution and 0.1 $\mathrm{M} \mathrm{KCl}$ as the supporting electrolyte. By applying the Randles-Sevcik equation (Eq. 3.5), ${ }^{174}$ we can determine and compare the surface:

$$
I_{p}=2.69 \times 10^{5} A \times D^{1 / 2} n^{3 / 2} v^{1 / 2} C
$$

Where $I_{p}$ represents the peak currents of the gold electrode, $A$ is the electroactive surface area $\left(\mathrm{cm}^{2}\right), D$ is the diffusion coefficient $\left(7.60 \times 10^{-6} \mathrm{~cm}^{2} \mathrm{~s}^{-1}\right),{ }^{175} n(=1)$ is the number of electrons transferred in the $\left[\mathrm{Fe}(\mathrm{CN})_{6}\right]^{3-/ 4-}$ redox reaction, $v$ is the scan rate $\left(\mathrm{v} \mathrm{s}^{1}\right)$, and $C$ is the concentration of the test solution $\left.(\mathrm{mol} \mathrm{cm})^{3}\right)$. Using constant parameters for $n$, $D$, and $C,{ }^{176}$ the real surface area of the AuEs could be determined. According to the 
Randles-Sevcik equation, the average electroactive surface area of the AuEs was $2.27 \mathrm{~mm}^{2}$

\section{(Figure 3.8).}
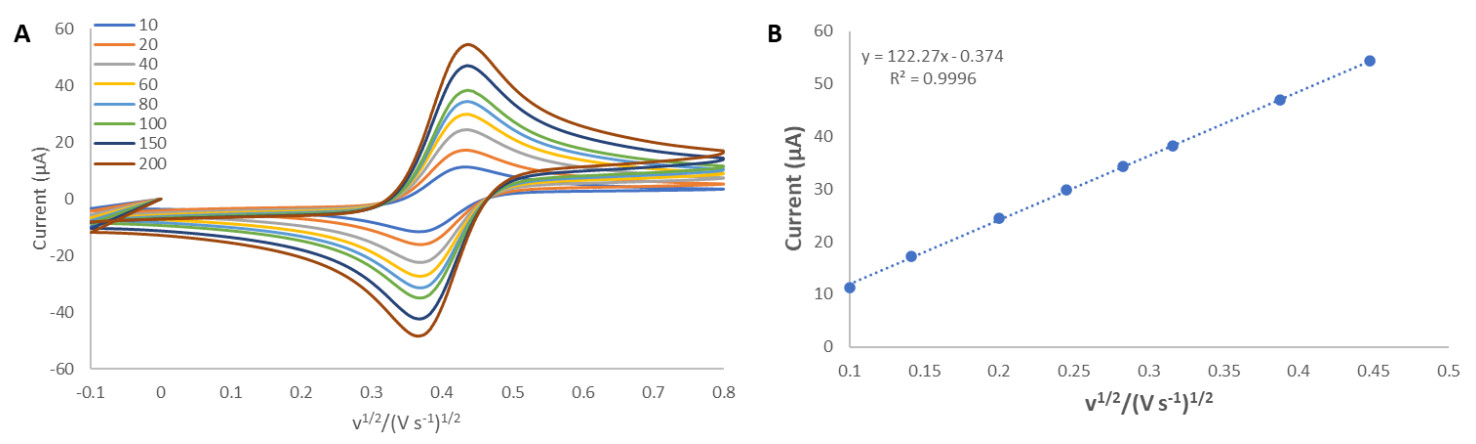

Figure 3. 8: (A) Cyclic voltammogram depicting the available surface area of a gold electrode over a scan rate of $10 \mathrm{mV}$ to $200 \mathrm{mV}$, a potential range of $-0.1 \mathrm{~V}$ to $0.8 \mathrm{~V}$, and in the presence of an electrolyte consisting of $5 \mathrm{mM}\left[\mathrm{Fe}(\mathrm{CN})_{6}\right]^{3-14-}$ and $0.1 \mathrm{M} \mathrm{KCl}$ in BFR-1. (B) calibration curve illustrating the gold surface area over varying scan rates $(10 \mathrm{mV}$ to $200 \mathrm{mV})$ where the Randles-Sevcik equation (Eq. 3.5) was applied to determine an average gold electrode surface area of $2.27 \mathbf{m m}^{2}(\mathrm{n}=4)$.

The aptasensor buffer and salt conditions, DNA concentration and immobilization times, $\mathrm{MCH}$ surface passivation, and aptasensor washing steps were optimized in that order to determine the optimal parameters necessary to fabricate a successful aptasensor. Phosphate buffered saline (PBS, 1x) is commonly used in biochemical research and was the primary buffer for all electrochemical experimentation. PBS, referred to as BFR-1, maintained a $\mathrm{pH}$ of 7.4 and contained a combination of $\mathrm{NaCl}, \mathrm{KH}_{2} \mathrm{PO}_{4}, \mathrm{~K}_{2} \mathrm{HPO}_{4}$, and initially $\mathrm{MgCl}_{2}$ to mimic the osmolarity, $\mathrm{pH}$, and ion concentration found within the physiological environment. PBS was the buffering solution when ASYN(1-5) were originally selected for monomeric $\alpha$-synuclein and allowed ASYN(1-5) aptamers to adopt a stable binding conformation in the presence of their target.

In order to immobilize thiolated aptamer on the AuE surface, a disulfide reducing agent would need to be applied to reduce the disulfide bond modification within the 5 ' end of the aptamer. Two commonly used disulfide reducing agents are DTT and TCEP, 
illustrated in Figure 3.9, result in the formation of reduced dithiols from the oxidized disulfide.

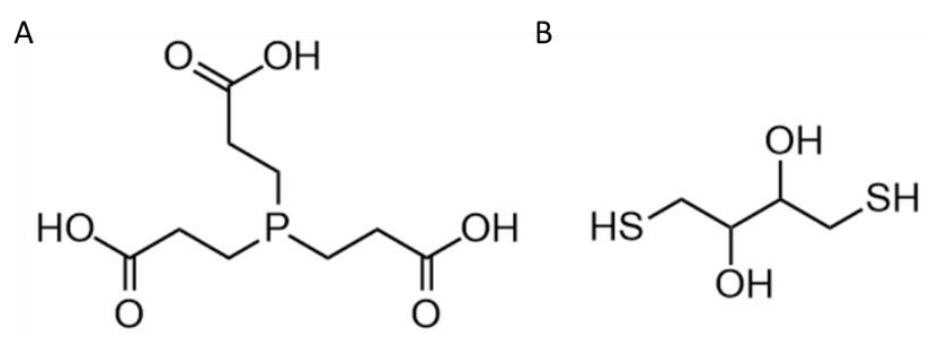

Figure 3. 9: Molecular structures of (A) TCEP and (B) DTT.

DTT is commonly used in biochemical research as an enzyme stabilizing agent and, in the case of DNA, reduction of disulfides via an $\mathrm{S}_{\mathrm{N}} 2$ mechanism. ${ }^{177}$ TCEP is another commonly used reducing agent that utilizes its strong phosphorus-oxygen bond to irreversibly reduce disulfides. ${ }^{178}$ TCEP was the preferred reducing agent of choice as it hosts a range of advantages over DTT. TCEP is odorless, non-volatile, and incredibly reactive with disulfide bonds over a broad range of $\mathrm{pH}$ values. The most ideal characteristic about TCEP over DTT is that it can remain in the immobilization solution without the need for its removal. Unlike DTT, TCEP does not possess any thiol groups that will competitively interact with the gold surface in the presence of thiolated DNA. TCEP is naturally acidic, having an approximate $\mathrm{pH}$ of 2.5 when dissolved in water. It was initially thought that its presence with DNA would affect surface immobilization due to its acidic nature, but the high salt concentration within PBS helped maintain the necessary neutral conditions. ${ }^{177}$ Although TCEP does not retain long-term stability in PBS, it is more stable than DTT in the presence of oxygen.

Following TCEP cleavage, the immobilization solution was heated to $95^{\circ} \mathrm{C}$ for 5 minutes prior to surface functionalization to denature the DNA and allow it to reform in its 
native . In the absence of this step inconsistent monolayer formation occurred, resulting in changes with the corresponding $R_{c t}$ value during EIS measurements. This inconsistency could be a result of the DNA strands forming a duplex through potential base pairing sites. A duplex on the AuE surface would not only alter the individual strands conformation and freedom in solution, but the presence of a duplex would significantly alter the charge on the surface by doubling the anionic charge experienced and limit the eventual binding of the target. ${ }^{179}$

The second parameter to consider was the fabrication of the DNA monolayer on the AuE surface. There are various methods throughout literature that use a broad range of parameters to develop the basis of their aptasensor including the coimmobilization of DNA and $\mathrm{MCH}$, concentration of immobilized DNA, the time necessary for successful DNA immobilization, and presence of $\mathrm{Mg}^{2+}$ ions in the immobilization buffer. Several studies ${ }^{179-}$ 183 suggest simultaneously coimmobilizing thiolated aptamer with surface passivating agent to increase AuE surface density through the formation of a more uniform and compact monolayer. It has been discussed previously that the role of the passivating agent is to displace non-specific interactions between the gold surface and immobilized DNA. By having $\mathrm{MCH}$ present during DNA immobilization, it can induce DNA to adopt a more linear conformation from the surface through its polar $\mathrm{OH}^{-}$head group and allow an increased DNA surface concentration to be fabricated. Figure 3.10 illustrates the 5'disulfide DNA modification where the DNA and $\mathrm{MCH}$ components are labelled following cleavage under reducing conditions. 


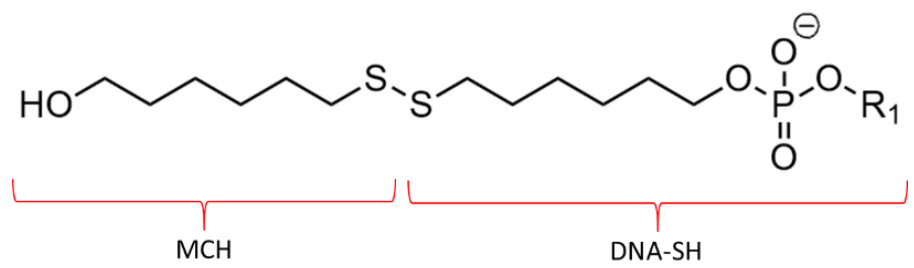

Figure 3. 10: Illustration of C6-S-S phosphoramidite modification at the 5'-terminal of ASYN(1-5). Cleavage in the presence of excess DTT or TCEP results in the formation of MCH and thiolated ssDNA in a 1:1 ratio, allowing for optimal coimmobilization of the gold surface during aptasensor optimization.

The SAM composition formed through coimmobilization is greatly influenced by the mole ratio of thiolated DNA to total thiol concentration in the immobilization solution. One study observed a linear increase in DNA surface density from $1.3 \times 10^{12} \mathrm{~cm}^{2}$ to $9.1 \mathrm{x}$ $10^{12} \mathrm{~cm}^{2}$ as the DNA fraction was increased from $0.2 \%$ to $50 \%$ respectively. ${ }^{183}$ TCEP does not contribute to the total thiol concentration, and the disulfide modification reduction yields thiolated DNA (DNA-SH) and an $\mathrm{MCH}$ molecule in a 1:1 ratio. At lower ratios, $\mathrm{MCH}$ cannot effectively compete with DNA, resulting in minimal surface coverage. As the ratio of $\mathrm{MCH}$ increases, $\mathrm{MCH}$ can readily assemble on the AuE surface and increase DNA spacing for optimal sensing. Several molecules have been used in place of $\mathrm{MCH}$ in literature, such as 2-mercaptoethanol (MCE), 3-mercaptopropionic acid (MPA), dithiothreitol (DTT), and oligo (ethylene glycol) (OEG). One study explored the use of binary and ternary SAMs to improve reactivity, stability, and orientation of the surface while minimizing signal-to-noise characteristics of the sensor background. ${ }^{180}$ It was discovered that through a two-step sensor fabrication where coimmobilization of DNA and DTT followed with $\mathrm{MCH}$ surface passivation, an aptasensor can be fabricated with extreme detection limits in the femtomolar range (fM) can be achieved. The combination of cyclic 
and linear backfilling agents minimizes surface defects and establishes a denser monolayer. ${ }^{180}$

The DNA concentrations and incubation times were optimized separately by plotting their respective $R_{c t}$ values. Figure 3.11A shows the DNA concentration necessary to effectively immobilize the surface over a concentration range of $1 \mu \mathrm{M}-20 \mu \mathrm{M}$ with a plateau occurring at $10 \mu \mathrm{M}$. Higher concentrations of DNA at $10 \mu \mathrm{M}$ provided consistently high $\mathrm{R}_{\mathrm{ct}}$ values, but upon target exposure, an insignificant change in transfer resistance occurred. It was hypothesized that the corresponding monolayer was too rich with DNA that in the presence of monomeric $\alpha$-synuclein, DNA might not be able to adopt its binding conformation. As a result, $1 \mu \mathrm{M}$ DNA concentration was used for surface fabrication. The incubation time necessary for monolayer formation is illustrated in Figure 3.11B over a period of $15 \mathrm{~min}$ to $20 \mathrm{~h}$. It is observed that surface coverage increases rapidly until $2 \mathrm{~h}$ where surface coverage is approximately $80 \%$ that of the full $20 \mathrm{~h}$ incubation period.
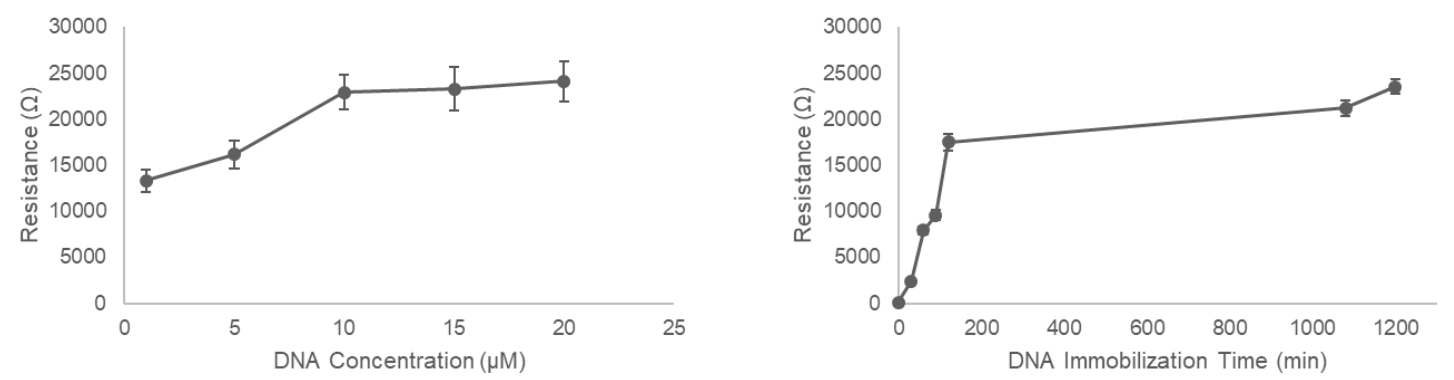

Figure 3. 11: Aptasensor optimization of DNA immobilization on a gold electrode surface. (A) DNA surface density measured over $1 \mu \mathrm{M}$ to $20 \mu \mathrm{M}$ resulted in a plateau at $10 \mu \mathrm{M}$. This concentration range was predicted to produce a high-density monolayer that could not effectively interact with the target of interest. As a result, $1 \mu \mathrm{M}$ DNA was used for the immobilization stage. (B) DNA incubation times were measured between $30 \mathrm{~min}$ and $1200 \mathrm{~min}$, where optimal DNA incubation time was determined to be $120 \mathrm{~min}$.

In the interest of time and reproducibility, a $2 \mathrm{~h}$ incubation time was applied for aptasensor development which compares with literature data. ${ }^{173,184}$ It is suggested that 
smaller length aptamers (below 24) form a denser monolayer in a shorter period of time than longer aptamers (aptamers analyzed by EIS were 66 bases in length). Reasoning behind this is due to the formation of a thiol gold bond, having a chemisorption energy of $30 \mathrm{kcal} / \mathrm{mol} .{ }^{185}$ In shorter sequences, thiol-gold bond formation drives its reaction with the surface. With increasing length, the effect of the thiol group diminishes due to the increase in sites of nucleotide-gold adsorption.

The final parameter optimized regarding DNA monolayer formation was the presence of $\mathrm{Mg}^{2+}$ within the immobilization buffer. $\mathrm{Mg}^{2+}$ cations are an integral component of secondary structure formation within aptamer strands as they can associate and form stable complexes with the phosphate and carboxylate anions located along the DNA backbone and nitrogenous bases respectively. ${ }^{186}$ Through DNA complexation, $\mathrm{Mg}^{2+}$ can influence the conformation of the aptamer directly and indirectly due to its size and water coordination sphere. $\mathrm{Mg}^{2+}$ can coordinate with the N7 site of purines, the N3 site of pyrimidines, and the oxygen atoms along the phosphate backbone. ${ }^{187}$ BFR-1 initially contained $1 \mathrm{mM} \mathrm{Mg}^{2+}$ in an attempt to create a high-density SAM. It was observed that aptasensor detection limits decreased significantly upon target interaction when $\mathrm{Mg}^{2+}$ was present in the immobilization buffer. Studies support the formation of high-density monolayers ${ }^{188,189}$ where the tethered aptamer may be sterically hindered by surrounding monolayer. This may inhibit the formation of the necessary secondary structure that is responsible for its binding characteristics. Circular dichroism was performed on ASYN2 aptamer in the presence of various concentrations of $\mathrm{Mg}^{2+}$ in BFR-1 as this aptamer was determined to have the highest affinity for monomeric $\alpha$-synuclein during the selection process. Figure 3.12 depicts circular dichroism spectra of monomeric $\alpha$-synuclein. 


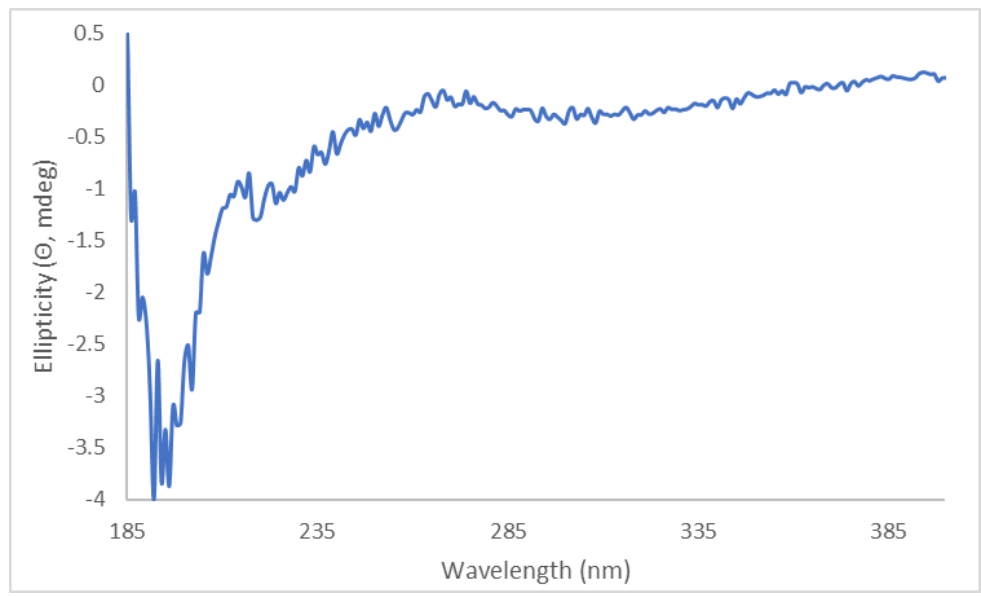

Figure 3. 12: CD spectra of monomeric $\alpha$-synuclein. The characteristic presence of a negative peak at $190 \mathrm{~nm}$ indicates the protein retains a random coiled conformation when immersed in BFR-1, consistent with literature.

The native conformation of this protein remains unknown and is commonly described as intrinsically disordered, but monomeric $\alpha$-synuclein secondary structure retains two alpha helices which become apparent in its CD spectra. Closely resembling literature spectra, ${ }^{190}$ random coiled structure is visible in the far ultraviolet region with a negative peak at $195 \mathrm{~nm}$.

Figure 3.13A illustrates the CD spectrum of the ASYN2 aptamer in the presence of varying concentrations of $\mathrm{Mg}^{2+}$ in BFR-1. ASYN2 (blue curve) in the absence of $\mathrm{Mg}^{2+}$ forms a parallel-stranded G-quadruplex, consistent with previous experimental data, with strong positive ellipticity at $270 \mathrm{~nm}$, followed by a weak negative peak at $245 \mathrm{~nm}$, and a weak positive peak at $210 \mathrm{~nm}$. As the concentration of $\mathrm{Mg}^{2+}$ is increased from $1 \mathrm{mM}$ to $100 \mathrm{mM}$ the B-DNA characteristics emerge, observed from the increase in strength of the negative peak at $245 \mathrm{~nm}$. The strength of the $210 \mathrm{~nm}$ peak, associated with the formation of a G-quadruplex secondary structure, diminished greatly with increasing $\mathrm{Mg}^{2+}$ ion concentration. This suggests that $\mathrm{Mg}^{2+}$ induces a conformational change within ASYN2 
that can be observed in Figure 3.13B when ASYN2 is subjected to monomeric $\alpha$-synuclein in varying concentrations of $\mathrm{Mg}^{2+}$. The conformation of ASYN2 in the presence of target significantly changes with higher ion concentration. The loss of its G-quadruplex functionality may be responsible for a reduction in $\mathrm{R}_{\mathrm{ct}}$ impedance observed during EIS measurements. Potential loss of secondary structure and formation of high-density monolayers support the removal of $\mathrm{Mg}^{2+}$ from the immobilization buffer.
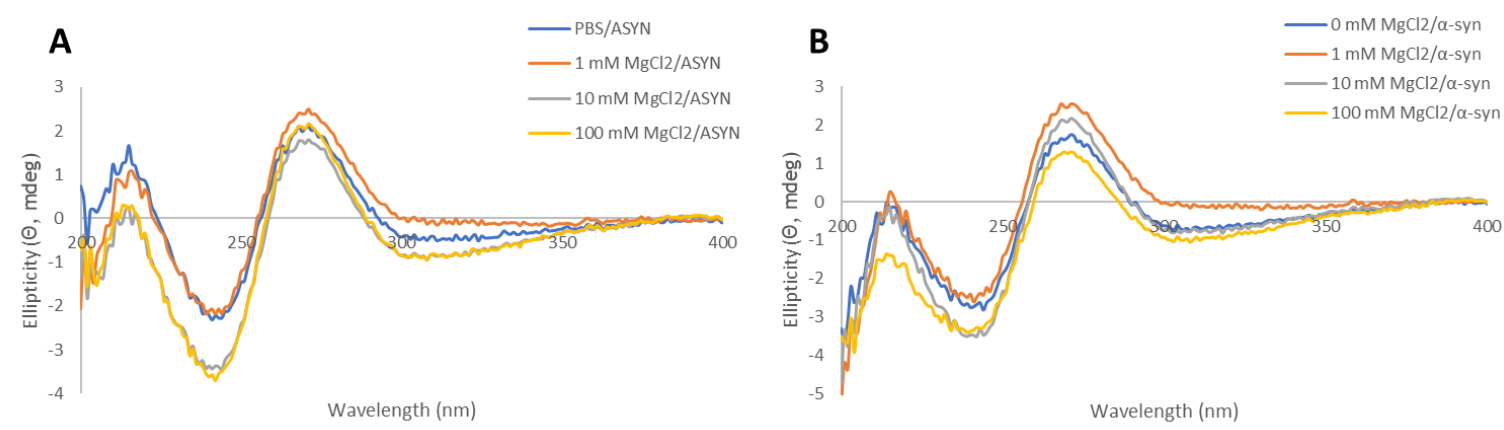

Figure 3. 13: Effect of BFR-1 containing varying $\mathrm{Mg}^{2+}$ concentrations ( $0 \mathrm{mM}$ to $100 \mathrm{mM}$ ) on secondary conformation of ASYN2. (A) $\mathrm{Mg}^{2+}$ in the absence of target influenced ASYN2 to become more structured as $\mathrm{Mg}^{2+}$ concentration increased to $10 \mathrm{mM}$. (B) $\mathrm{Mg}^{2+}$ in the presence of monomeric $\alpha-$ synuclein influenced ASYN2 to adopt greater B-DNA characteristics, increase in $270 \mathrm{~nm}$ and decreased in $240 \mathrm{~nm}$ peaks respectively, as $\mathrm{Mg}^{2+}$ concentrations were increased to $100 \mathrm{mM}$.

A reversed direction ASYN2 aptamer, named ASYN2R, was synthesized for EIS measurements to determine what structural regions within the 66-mer sequence are primarily responsible for binding with $\alpha$-synuclein (Figure 3.14).

During DNA synthesis, construction occurs in the $3^{\prime} \rightarrow 5^{\prime}$ direction from a solid support CPG column with a modifier being added last as a 5'-phosphoramidite. With regards to a 3'-modification, the modifier will need to be adsorbed to the CPG columns prior to the beginning of the synthesis cycle. Due to lack of commercial availability, a 3'C6 S-S modifier was not available. To compensate for this, the ASYN2 sequence was synthesized in reverse where the 5' end of ASYN2 acted as the 3' start of ASYN2R. This 
allows the secondary structures residing in the 3' and 5' primer binding regions to interact with $\alpha$-synuclein to determine if either, or both, are responsible for target interaction. One concern with ASYN2R was whether the aptamer's directionality would impact its ability to successfully interact with $\alpha$-synuclein, further explored in the subsequent section.

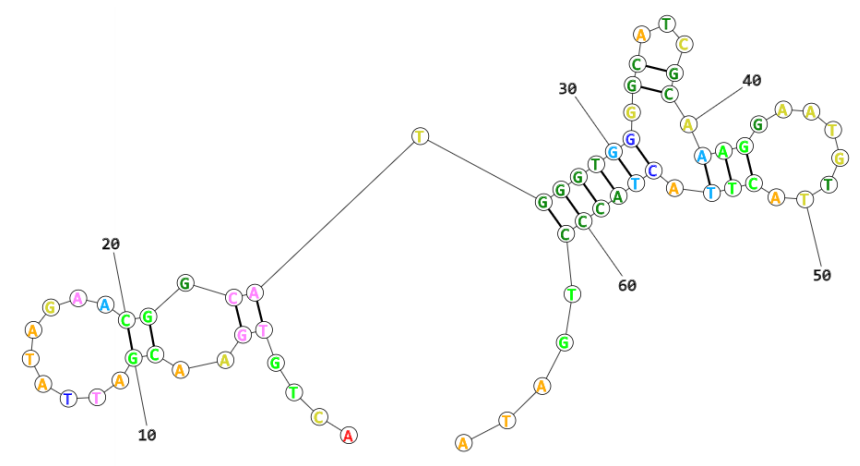

Figure 3. 14: Predicted secondary structure of ASYN2R according to RNAstructure. ASYN2R bears a close resemblance to ASYN2.

Following DNA tethering, $\mathrm{MCH}$ passivation was optimized where concentrations of $1 \mathrm{mM}$ to $15 \mathrm{mM}$ were examined to determine which is ideal for aptasensor construction. The results (Figure 3.15) indicate little change in resistance as the $\mathrm{MCH}$ was increased to $15 \mathrm{mM}$. This indicates that $1 \mathrm{mM}$ was already in excess and passivated the AuE accordingly. Problems in aptasensor fabrication would arise if the DNA-functionalized $\mathrm{AuE}$ was immersed in a lack of $\mathrm{MCH}$. In the event of low $\mathrm{MCH}$ concentrations, incomplete surface coverage is possible as non-specific DNA interactions with the gold surface could persist. During washing and target incubation stages, non-specific interactions could become disrupted, causing the DNA to adopt a more linear form off the surface and the formation of an unpassivated site. It is likely that lack of surface coverage would allow low impedance channels to form, leading to a decrease in observed $\mathrm{R}_{\mathrm{ct} .}{ }^{182}$ 


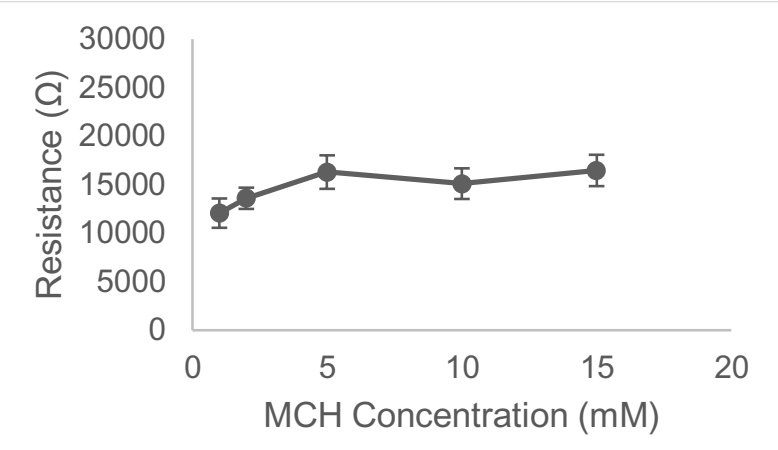

Figure 3. 15: Aptasensor optimization of MCH passivation on gold electrode surface. MCH concentrations between $1 \mathrm{mM}$ and $15 \mathrm{mM}$ provided no change in resistance, indicating that this concentration range is in excess and does not alter monolayer formation significantly with increasing concentration. $1 \mathrm{mM}$ MCH was determined to be optimal.

A rinsing buffer, BFR-3, was implemented prior to measuring the impedance of the aptasensor. ${ }^{173}$ The purpose of the rinsing buffer was to aid in removing non-specifically bound DNA and $\mathrm{MCH}$ that may be residing on the AuE. From Figure 3.16, electrodes were rinsed for 15 min and 30 mins prior to impedance measurement. It was observed that a decrease in recorded $R_{c t}$ occurred after a 15 min wash with no change in $R_{c t}$ when a 30 min wash was applied. The decrease in $\mathrm{R}_{\mathrm{ct}}$ can be associated with the formation of a uniform monolayer.

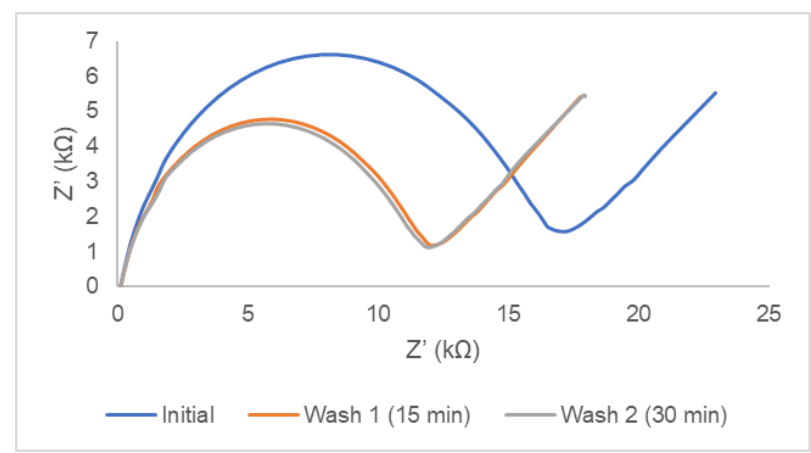

Figure 3. 16: Application of rinsing buffer (BFR-4) to remove non-specifically bound DNA and MCH from the gold electrode surface. It was determined that $15 \mathrm{~min}$ and $30 \mathrm{~min}$ washing steps are superimposed, indicating that 15 min was sufficient. 


\subsubsection{Monomeric $\alpha$-synuclein detection and specificity of the aptasensor}

Applying the optimized conditions to the aptasensor, its ability to detect monomeric $\alpha$-synuclein was measured over a range of concentrations where monomeric $\alpha$-synuclein was recognized by the aptasensor through weak interactions to form an aptamer- $\alpha$ synuclein complex. Figure 3.17 illustrates the corresponding Nyquist plots for the specific detection of ASYN2 in the presence of monomeric $\alpha$-synuclein $(0.001 \mu \mathrm{M}-1 \mu \mathrm{M})$.

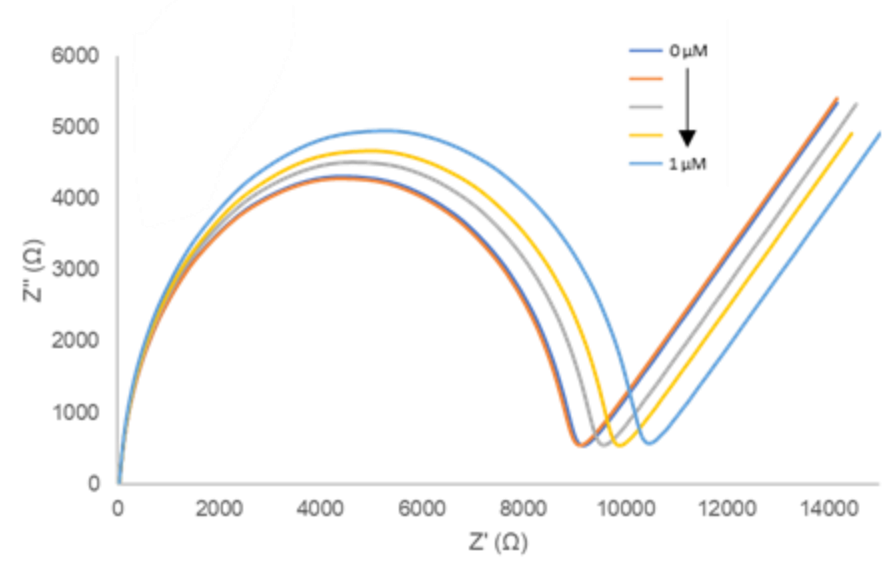

Figure 3. 17: Nyquist diagram of ASYN2 in the presence of monomeric $\alpha$-synuclein from $1 \mathrm{nM}$ to 1000 $\mathrm{nM}$ illustrated an increasing $\mathbf{R}_{\mathrm{ct}}$ with increasing target presence.

It is observed that through increasing target concentrations the $\mathrm{R}_{\mathrm{ct}}$ at the electrodeelectrolyte interface increases, suggesting the successful formation of an aptamer-target complex. A calibration curve was formed to determine the aptasensors LDR, and binding affinity $\left(\mathrm{K}_{\mathrm{d}}\right)$ for the respective sequences. The calibration curve (Figure 3.18) for ASYN(1-5) and ASYN2R sequences representing a single isotherm ( $\mathrm{N}=4$ electrodes) is expressed as $\Delta_{\text {ratio }}$ Vs protein concentration where $\Delta_{\text {ratio }}$ is defined as: ${ }^{.157}$

$$
\begin{aligned}
& \Delta_{\text {ratio }}=\Delta_{\mathrm{s}} / \Delta_{\mathrm{p}} \\
& \Delta_{\mathrm{s}}=\mathrm{R}_{\mathrm{ct}(\mathrm{ASYN}-\alpha \text {-synuclein) }}-\mathrm{R}_{\mathrm{ct} \text { (AuE-buffer })} \\
& \Delta_{\mathrm{p}}=\mathrm{R}_{\mathrm{ct}(\mathrm{ASYN})}-\mathrm{R}_{\mathrm{ct} \text { (AuE-buffer) }}
\end{aligned}
$$



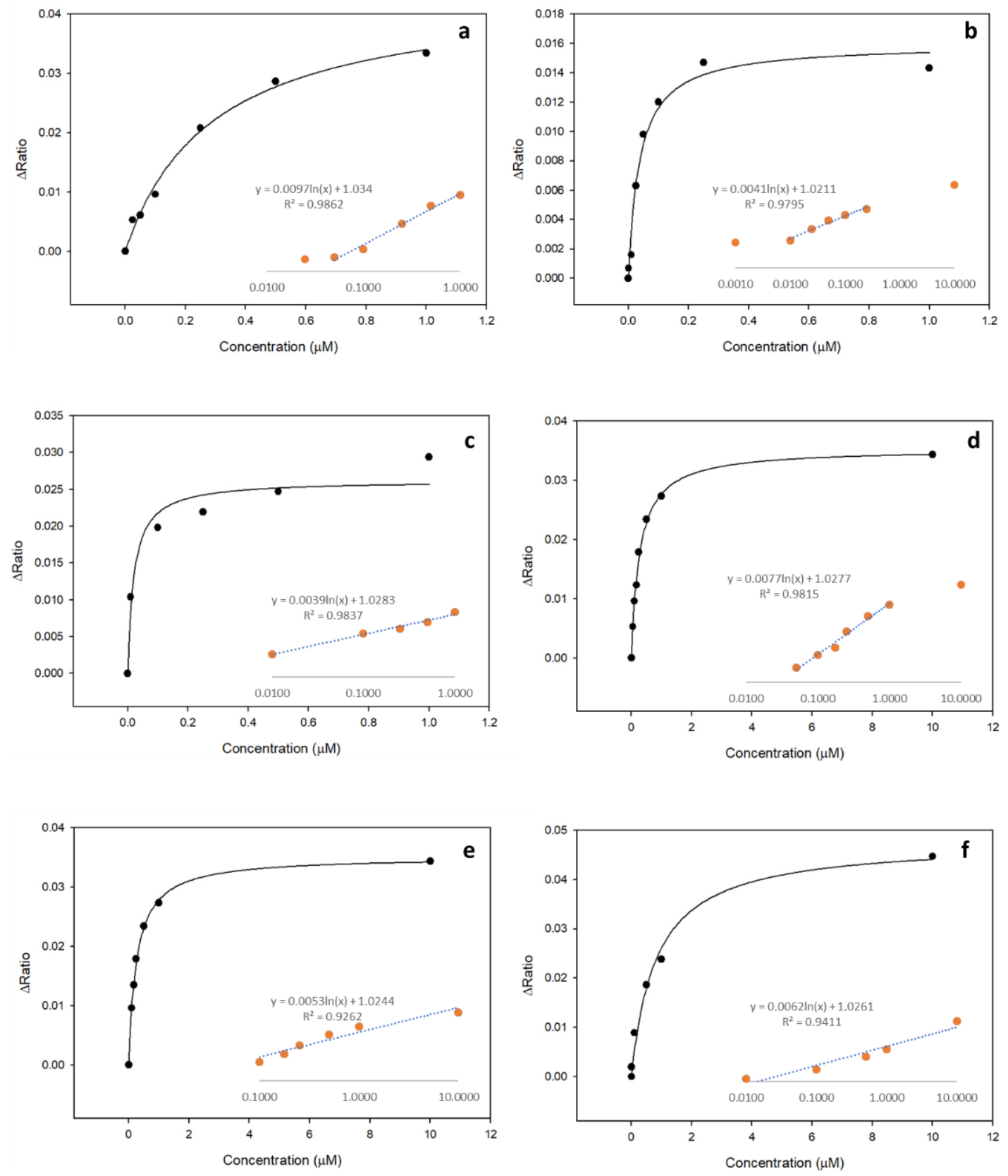

Figure 3. 18: EIS measurements illustrating the binding affinity of (a) ASYN1, (b) ASYN2, (c) ASYN2R, (d) ASYN3, (e) ASYN4, and (f) ASYN5 aptasensors in the presence of monomeric $\alpha$ synuclein. $K_{d}$ values were determined in SigmaPlot using nonlinear regression analysis. $\Delta R a t i o=\Delta S / \Delta P$

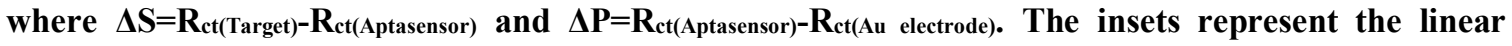
dynamic range of the respective aptasensors over the selected concentration range. 
By applying single ligand saturation regression analysis to the calibration curve, $\mathrm{K}_{\mathrm{d}}$ values between $20 \pm 9 \mathrm{nM}$ and $820 \pm 160 \mathrm{nM}$ were obtained with an average LDR from 10 $\mathrm{nM}$ to $1000 \mathrm{nM}$. Aptasensor results are summarized in Table 3.1 and compared to literature aptamers for both monomeric and oligomeric forms of $\alpha$-synuclein.

Table 3. 1: $K_{d}$ values for selected aptamers against monomeric $\alpha$-synuclein and compared to currently existing $\alpha$-synuclein aptamers in literature. Aptamers selected for monomeric protein are denoted with (a) and those selected for the oligomeric protein are denoted with (b).

\begin{tabular}{ll}
\hline Aptamer Sequence & $\mathrm{K}_{\mathrm{d}}(\mathrm{nM})$ \\
\hline ASYN1 $^{\mathrm{a}}$ & $290 \pm 43$ \\
\hline ASYN2 $^{\mathrm{a}}$ & $70 \pm 3$ \\
\hline ASYN2R $^{\mathrm{a}}$ & $20 \pm 9$ \\
\hline ASYN3 & $273 \pm 18$ \\
\hline ASYN4 & $260 \pm 11$ \\
\hline ASYN5 & $826 \pm 164$ \\
\hline M515 ${ }^{\mathrm{a} b}$ & $\mathrm{~N} / \mathrm{A}$ \\
\hline T-SO508 & \\
\hline T-SO530 & 68 \\
\hline F5R1 $^{\mathrm{b}}$ & 63 \\
\hline F5R2 $^{\mathrm{a}}$ & 2.40 \\
\hline
\end{tabular}

Interestingly, ASYN2R retains the highest affinity for $\alpha$-synuclein as opposed to the predicted ASYN2. Potential reasoning for this higher affinity is due to the exposed 5'PBR in ASYN2R compared to the anchored 5'-PBR in AYSN2. This suggests that specific regions may be responsible for the sequences binding properties with monomeric $\alpha$ synuclein as opposed to the ASYN2 sequence as a whole. However, the curve fitting of 
ASYN2R is rather poor and suggests a relatively high degree of error attributed to aptasensor detection. Due to the high potential error associated with ASYN2R, its binding affinity to monomeric $\alpha$-synuclein remains questionable and was not evaluated further. To investigate these findings, ASYN2 was truncated in various sections, termed minimers, to determine what conserved regions retain binding affinity for $\alpha$-synuclein and which potential segments within each region are necessary for these interactions. ASYN2 minimers are labelled $\mathrm{A} 2 \mathrm{~m}(1-6)$, corresponding to the six minimers that were designed from the parent structure. Minimer data will be further discussed in subsequent sections.

Within a healthy human body, $\alpha$-synuclein is present at concentrations of $5.6 \mathrm{mcg} / \mathrm{L}$ $(0.39 \mathrm{nM})$ in human plasma ${ }^{191}$ and $0.1 \mathrm{ng} / \mathrm{mL}$ to $1 \mathrm{ng} / \mathrm{mL}(0.0069 \mathrm{nM}-0.069 \mathrm{nM})$ in cerebrospinal fluid (CSF). ${ }^{192}$ With respect to the performed EIS studies ASYN2R displayed the highest affinity for $\alpha$-synuclein with an LOD of $20.2 \mathrm{nM}$, significantly higher than concentrations observed in healthy humans. This result would indicate that the tested electrochemical platform is not a suitable detection method of $\alpha$-synuclein in healthy humans. However, overexpression of $\alpha$-synuclein has been observed within neuronal cells along with the duplication and triplication of misfolded, or mutant, variants. It is possible that the LOD of EIS would be feasible in patients exhibiting late-stage PD as $\alpha$-synuclein concentrations would be significantly elevated with respect to someone experiencing early stage PD symptoms.

One study ${ }^{193}$ contrasting the concentration of phosphorylated $\alpha$-synuclein, PS-129, in the CSF of sick vs healthy individuals found that PS-129 was significantly higher in PD patients $(79.23 \pm 23.22 \mathrm{pg} / \mathrm{mL})$ vs healthy individuals $(68.61 \pm 17.25 \mathrm{pg} / \mathrm{mL})$. PS-129 is a PTM within $\alpha$-synuclein that has been associated with increased $\alpha$-synuclein toxicity both 
in vivo and in vitro. PS-129 may influence the binding interaction between ASYN2 and $\alpha$ synuclein, but concentrations of this magnitude are still below the threshold of what this aptamer is capable of detecting with this sensing platform. This was the anticipated outcome for the designed aptasensor as the goal of this aptamer was not for sensing purposes but rather for target inhibition. Higher monomeric $\alpha$-synuclein concentrations were implemented during ASYN(1-5) SELEX as these higher protein concentrations are more representative of what will be experienced physiologically, allowing for better protein inhibitors to be selected at the loss of stronger binding affinity.

To determine the selectivity of the aptasensor, the $\mathrm{R}_{\mathrm{ct}}$ response was measured against proteins of similar structure (Figure 3.19) such as $\alpha$-synuclein fibril, $\alpha$-synuclein A53T mutant, $\beta$-synuclein, $\gamma$-synuclein, and thrombin at identical concentrations of $1 \mu \mathrm{M}$. The first alternative target to be analyzed was the $\alpha$-synuclein A53T mutant protein, a missense point mutation where an alanine (Ala) amino acid is changed to a threonine (Thr) at the $53^{\text {rd }}$ position within the protein (Figure 3.20).

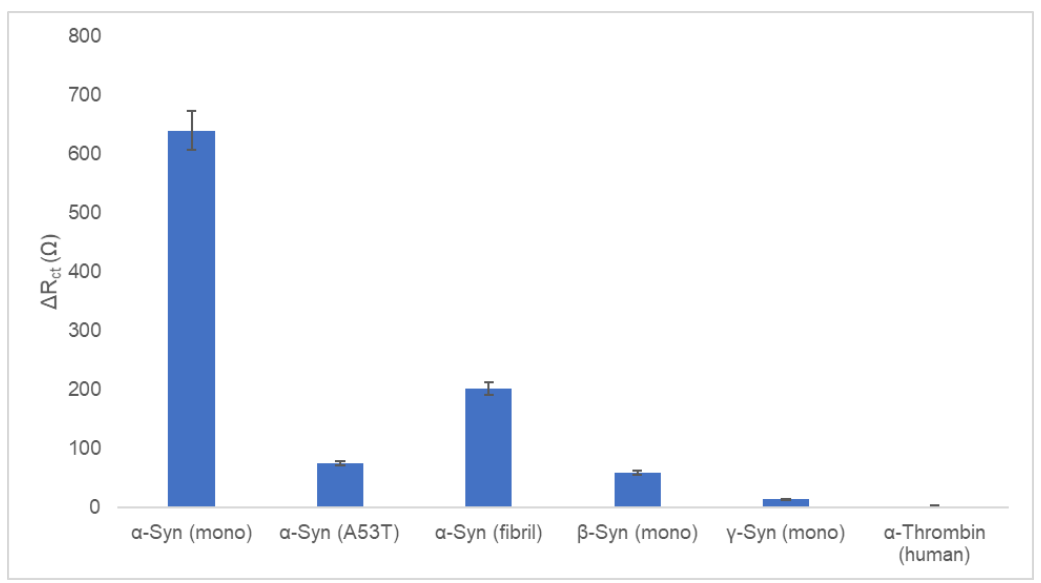

Figure 3. 19: Selectivity of ASYN2 aptasensor in the presence of $1 \mu \mathrm{M}$ of the respective targets. ASYN2 retained high affinity for monomeric forms of $\alpha$-synuclein while showing unexpectedly low affinity for the A53T mutant variant. 
A53T was determined to be an autosomal dominant trait that has been linked to early-onset PD and undergoes fibrillization at a faster rate than monomeric $\alpha$-synuclein. ${ }^{2}$ It was initially predicted that the A53T mutant variant would impede electron transfer to a similar extent as the monomeric protein due to similar structure with the exception of a single AA.

MDVFMKGLSKAKEGVVAAAEKTKQGVAEAAGKTKEGVLYVGSKTKEGVVHGVATVAEKTKEQVTNVGGAVVTGVTAVAQKTVEGAGSIAAATGFVKKDQLGKNEEGAPQEGILEDMPVDPDNEAYEMPSEEGYQDYEPEA MDVFMKGLSKAKEGVVAAAEKTKQGVAEAAGKTKEGVLYVGSKTKEGVVHGVITVAEKTKEQVTNVGGAVVTGVTAVAQKTVEGAGSIAAATGFVKKDQLGKNEEGAPQEGILEDMPVDPDNEAYEMPSEEGYQDYEPEA

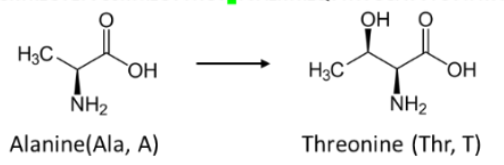

Figure 3. 20: Protein sequence of A53T $\alpha$-synuclein. Molecular structure of alanine (Ala,A) and threonine $(\mathrm{Thr}, \mathrm{T})$ that underwent a missense point mutation at the $53^{\text {rd }}$ position. The substitution of a methyl for a hydroxyl appears to significantly alter binding interactions.

However, the results indicate an 8 -fold reduction in $\mathrm{R}_{\mathrm{ct}}$ with a respective value of $75 \Omega$ compared to a $R_{\text {ct }}$ of $640 \Omega$ for monomeric $\alpha$-synuclein. The substitution of a methyl group for a hydroxyl group in the AA sequence could alter binding between aptamer and target as the increase in polarity would reduce hydrophobic effects at that site, potentially causing structural alterations within the protein that inhibit effective aptamer binding. Due to its tendency to undergo fibrillization, a TEM of the A53T protein (Figure 3.21) was performed to determine if the protein existed as a monomer or fibril at the time of analysis.

From the TEM it is very clear that excessive fibrillization had occurred prior to electrochemical testing. The formation of fibrils would result in a lower $\mathrm{R}_{\mathrm{ct}}$ as the aptamer was not selected for this protein conformation, and the presence of monomeric forms of the protein would be significantly reduced in the incubation solution. The small $\mathrm{R}_{\mathrm{ct}}$ value obtained is most likely a result of minimal monomeric protein remaining in solution, or from the aptasensor interacting with exposed monomeric sites within the fibril complex. 
An additional experiment on unaggregated A53T protein would need to be performed to accurately assess ASYN2s affinity for this target.

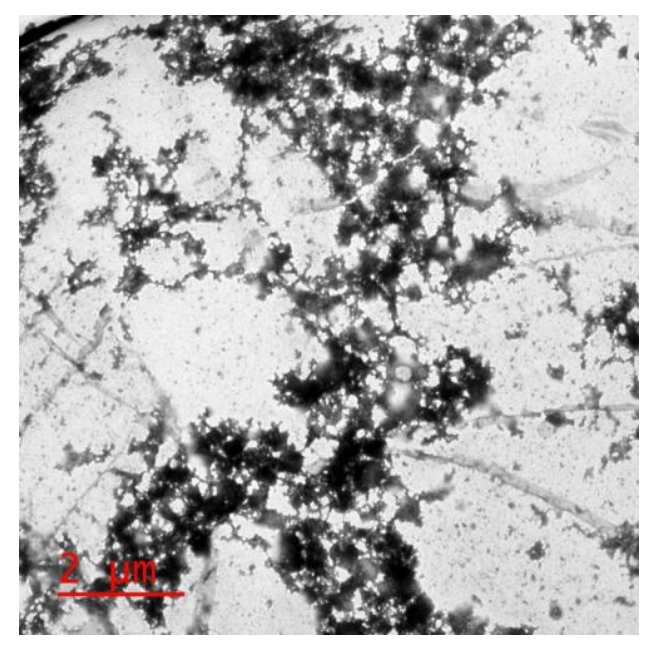

Figure 3. 21: TEM of A53T protein indicating the extensive aggregation that had occurred prior to electrochemical analysis.

Fibril complexes were formed in vitro according to Experimental 2.12 using monomeric $\alpha$-synuclein in the presence of a seed peptide to induce fibrillization over a seven-day period. Fibril formation is a result of endogenous aggregation of monomeric $\alpha$ synuclein, seeding the progression of Lewy Bodies (LB) and Lewy Neurites (LN), making it a potential biomarker for diagnosis and development of molecular therapeutics to treat PD. The $\mathrm{R}_{\mathrm{ct}}$ of the aptasensor indicated a larger electron transfer resistance than expected with a $R_{c t}$ value of $202 \Omega$ when compared to its monomeric substituent, but still remained less sensitive as expected. There are several potential reasons that can explain the aptasensors affinity for the fibril complex. Ultracentrifugation or gel separation was not performed on the fibril sample prior to binding analysis. It is possible that some proportion of monomeric $\alpha$-synuclein did not undergo fibrillization and remains in solution as a free monomer, interacting with the aptasensor and yielding false-positive results. It is also 
possible that during fibrillization the overall monomer conformation was not altered, leaving the binding site available for target interaction.

The next two targets measured were a part of the synuclein family, being $\beta$ synuclein and $\gamma$-synuclein. Protein sequences are compared in Figure 3.22 where highly conserved amino-terminal domains are retained among the three sequences, but major alterations and deletions are pronounced in the carboxy-terminal domain. ${ }^{21} \beta$-synuclein and $\gamma$-synuclein were also identified within CSF in concentrations of $6.87 \mathrm{pM}-43.9 \mathrm{pM}$ and $128 \mathrm{pM}-774 \mathrm{pM}$ respectively. ${ }^{194}$

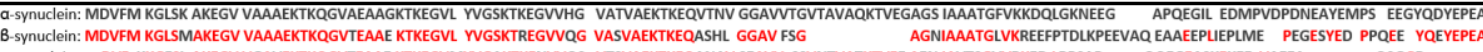

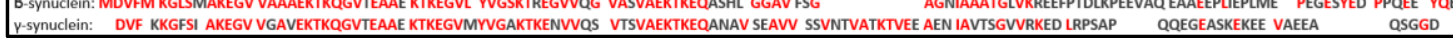

Figure 3. 22: Protein structure comparisons between $\alpha$-synuclein with $\beta$-synuclein and $\gamma$-synuclein respectively. Similarities across protein sequences have been highlighted in red and were performed using Clustal Omega.

The roles of these two proteins are less studied than $\alpha$-synuclein, where $\beta$-synuclein has been suggested as a neuroprotective agent responsible for inhibiting $\alpha$-synuclein aggregation, while $\gamma$-synuclein has been linked with neurodegeneration and has been identified as a potential biomarker for breast and ovarian cancers. ${ }^{21,194}$ Electrochemical analysis recorded low $R_{\text {ct }}$ values for the two proteins with $\beta$-synuclein having a $R_{c t}$ of 60 $\Omega$ and $\gamma$-synuclein of $15 \Omega$. This low impedance value in comparison to $\alpha$-synuclein is most likely stemming from conformational changes and AA availability within the protein structures. $\alpha$-synuclein is $140 \mathrm{AA}$ in length, while $\beta$ - and $\gamma$-synuclein are 134 and 127 AAs respectively. The loss of AAs in $\beta$ - and $\gamma$-synuclein is predominantly in the carboxydomain where minimal structural similarities are observed with $\alpha$-synuclein. These findings suggest that the carboxy-domain is a potential site of interaction for ASYN2. 
One study that could be performed to investigate the structure-dependent nature of ASYN2 for $\alpha$-synuclein is to analyze its binding affinity for the individual regions within the protein. As $\alpha$-synuclein is composed primarily of three regions: An N-terminal amphipathic domain, a non-amyloid component (NAC)-domain, and a carboxy-terminal domain, truncated forms of $\alpha$-synuclein could be analyzed individually to determine which segment the aptamer preferentially binds with. Various truncated peptides are available, such as sequences $1-60,1-95,103-129,61-140$, and 96-140. This variety of sequence lengths containing various segments and conserved regions could isolate the active site(s) within the protein, allowing for further enhancement of the aptamer or improvement of its therapeutic potential by uncovering a potential mechanism of interaction.

The last protein comparison performed was on $\alpha$-Thrombin, an integral component of the blood clotting cascade through its conversion of fibrinogen to fibrin. ${ }^{157}$ As expected, the impedance for this protein was minimal with a recorded $R_{c t}$ of $3 \Omega$. Having minimal structural similarities with $\alpha$-synuclein, this indicates that ASYN2 displays specificity for its monomeric target.

One of the challenges with determining a $\mathrm{K}_{\mathrm{d}}$ for the respective ASYN aptamers is the steric hindrance that accompanies them while anchored to the AuE. The lack of freedom from being tethered on one end does not accurately represent their $\mathrm{K}_{\mathrm{d}}$ for the target when compared with free aptamer in solution. Unlike the initial SELEX conditions where ASYN(1-5) existed as a free aptamer in solution, tethered ASYN(1-5) exhibited significantly higher selectivity for monomeric $\alpha$-synuclein. One line of reasoning to explain these results focuses on receptor clustering and electrode surface composition. Immobilized sequences in the vicinity of an adjacent aptamer- $\alpha$-synuclein complex may 
immediately interact with $\alpha$-synuclein upon its release. This would imply better binding affinity as a result of slower $\mathrm{k}_{\text {off }}$ kinetics. The fact that SELEX conditions were not conducted under protein concentrations in this range also suggests that as a free aptamer, ASYN(1-5) may not be able to effectively recognize $\alpha$-synuclein at this concentration.

As a result, tests should be performed using various techniques to determine a $\mathrm{K}_{\mathrm{d}}$ for monomeric $\alpha$-synuclein that examines both its immobilized and free states. One paper in particular ${ }^{195}$ examines the specific challenges of EIS with regards to protein biosensing. The group identified various methods commonly used throughout literature that could lead to false-positive $R_{c t}$ results such as repetitive measurements with the same electrode, measuring EIS and CV with the same electrode, and buffer incubations in between EIS measurements. ${ }^{195}$ Optimization of aptasensor parameters were put in place to mitigate the effects of these potential errors but illustrates the importance of having multiple experimental methods to obtain an aptamer $\mathrm{K}_{\mathrm{d} .}{ }^{196}$ Successive ASYN $\mathrm{K}_{\mathrm{d}}$ experiments were performed using circular dichroism (CD), microscale thermophoresis (MST), and colorimetric assays.

\subsection{Microscale thermophoresis (MST) of ASYN2}

Microscale thermophoresis (MST) is an attractive technique for analyzing biomolecular interactions of free species in solution, providing sensitive detection through the thermodiffusion of fluorescently labelled ASYN2 aptamer with its protein target. ${ }^{197}$ Thermodiffusion, also known as the Soret effect, can be described as the behavioural change of a molecular species subjected to a temperature gradient that induces molecular movement. ${ }^{198}$ There are several factors that can influence thermophoretic movement such 
as molecular size, conformation, ionic charge, and the temperature-dependent nature of the species being investigated. ${ }^{197,198}$ As a result of the above parameters, the accumulation or depletion of molecules towards or away from a region of altered temperature will ensue. The basic MST setup is illustrated in Figure 3.23. ${ }^{199}$
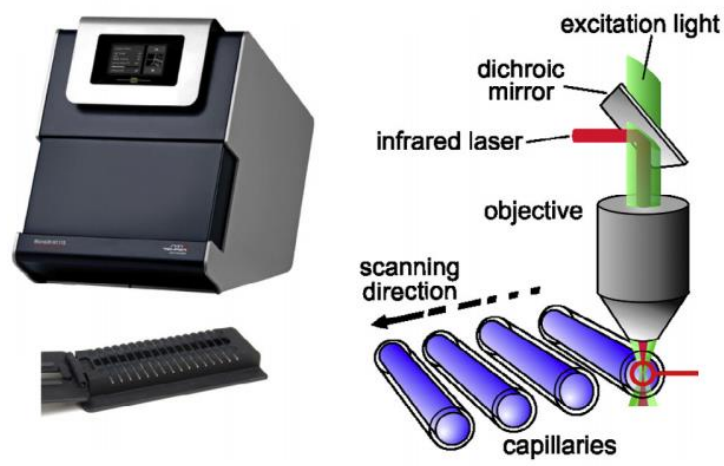

Figure 3. 23: MST experimental setup. ${ }^{199}$

Here, an IR-laser is focused on a glass capillary containing the Cy5-modified ASYN aptamer and protein target. As the IR induces a temperature gradient, the thermophoretic movement of fluorescently-labelled DNA in both bound and unbound states can be differentiated through the intrinsic fluorescence excitation of the Cy5 modifier. The IR-laser is coupled to fluorescence detection software along the same optical path to simultaneously analyze local temperature differences with its respective fluorescence.

A fluorescence time trace of an MST experiment is depicted in Figure 3.24 where the localized thermodynamic equilibrium for a DNA-protein sample is measured due to the fluorescence migration that occurs under an IR-induced temperature change. ${ }^{200}$ The time trace can be separated into five regions, labelled (I)-(V). 


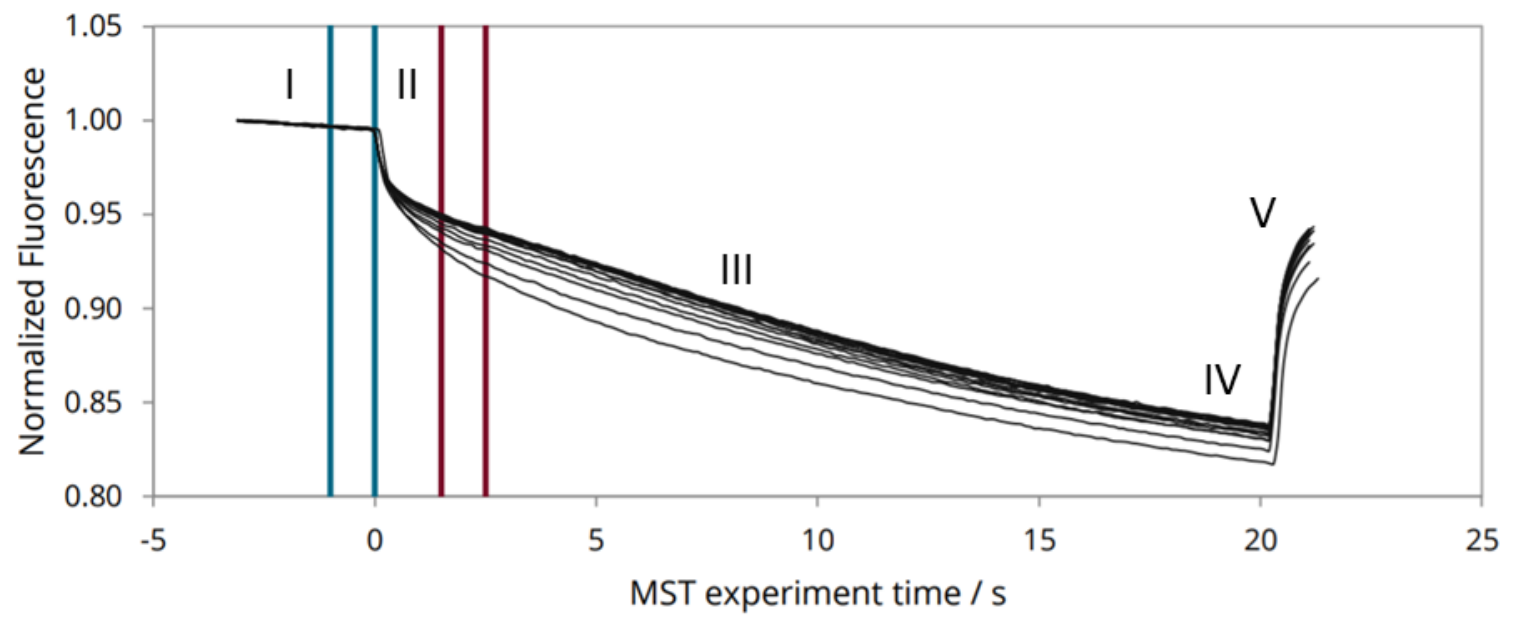

Figure 3. 24: MST fluorescence timetrace depicting different phases of the experiment: the initial fluorescence of ASYN2-Cy5 (I), IR laser activation induces a temperature jump and decreases fluorescence (II), thermophoresis occurs as unbound and bound complexes migrate from the heated region until a steady state is achieved (III), deactivation of the IR laser causes a second temperature jump to occur (IV), diffusion of species into depleted region occurs to increase solution homology (V). The blue region denotes the cold period prior to laser activation and the red region denotes thermophoretic migration upon laser activation. As higher concentrations of ligand are titrated into the solution containing ASYN2-Cy5, the respective timetrace changes as a result. ASYN2 initially begins as an unbound aptamer and gradually plateaus in a bound aptamer-target complex.

Region (I) symbolizes the initial state of the solution prior to IR-heating where a uniform fluorescence is recorded due to the random movement of DNA and protein in solution, referred to as Brownian motion. When the IR-laser is initiated, an immediate drop in fluorescence is recorded within $<100 \mathrm{~ms}$ (II) and is known as a temperature jump. This fluorescence decrease is strictly due to the temperature change within the local environment, causing thermodiffusion of ASYN2 in its bound and unbound states to occur. Application of a temperature gradient induces ASYN2 to migrate from a hot region to a cold region, down the temperature gradient. ASYN2-target complexes will migrate at a different rate than unbound ASYN2 and can be easily distinguished through their normalized fluorescence. Thermophoresis is observed $>1 \mathrm{~s}$ (III) where a steady decrease in fluorescence results from molecular migration away from the heated region. A steady- 
state is reached between molecules undergoing thermodiffusion and normal diffusion prior to IR-laser removal (IV). This results in a reversed temperature jump where fluorescence rapidly increases as back-diffusion occurs to restore the concentration imbalance and steady-state in solution (V). ${ }^{201,202}$ By maintaining a constant concentration of fluorescently labelled ASYN2, the initial fluorescence remains constant between serial dilutions of the protein target of interest. This is denoted in Figure 3.24 due to the initial steady state (cold area, blue region) labelled as $F_{0}$ and thermodiffusion following laser activation (hot area, red region) labelled as $F_{l}$.

Figure 3.24 illustrates the MST binding curve of ASYN2-Cy5 in the presence of monomeric $\alpha$-synuclein over a concentration range of $305 \mathrm{pM}$ to $10 \mu \mathrm{M}$. As the concentration of protein target is increased, the degree of binding can be observed when comparing the fluorescence emitted between the unbound plateau (low $\alpha$-synuclein concentrations) and the bound plateau (high $\alpha$-synuclein concentrations).

Four targets were analyzed in the presence of ASYN2: monomeric $\alpha$-synuclein, $\alpha$ synuclein A53T mutant, $\beta$-synuclein, and $\gamma$-synuclein and their respective normalized MST traces are illustrated in Figure 3.25A-D. ASYN2 concentration was kept constant for each measurement at $50 \mathrm{nM}$ and respective target concentrations were titrated over the range of $305 \mathrm{pM}$ and $10 \mu \mathrm{M}$. When comparing the four MST traces, a plateau is apparent within the lowest concentrations of titrated protein for all respective proteins while there is not a visible plateau at the highest protein concentrations. This is due to insufficient quantities of protein to explore this region and to the fact that protein concentrations in excess of the measured region are not practical as they are expensive and would not be observed in a physiological setting. Exploring lower protein concentrations would also not contribute to 
a change in steady-state and provide very limited results. By analyzing the change in $F_{n o r m}$, the affinity of ASYN2 for the four targets can be determined.
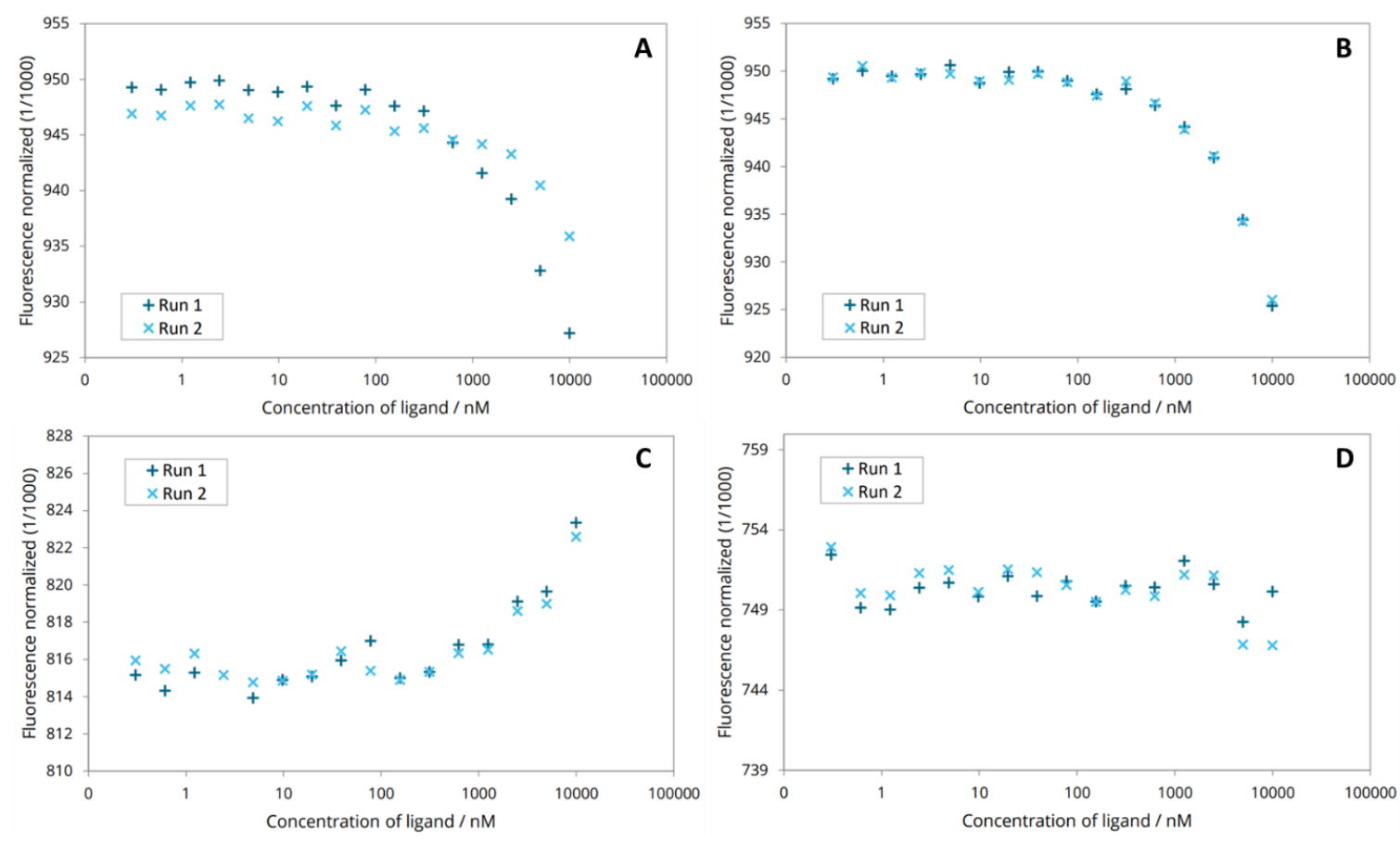

Figure 3. 25: MST detection of ASYN2 $K_{d}$ in the presence of different synuclein structures: (A) monomeric $\alpha$-synuclein, (B) A53T mutant $\alpha$-synuclein, (C) $\beta$-synuclein, (D) $\gamma$-synuclein. The normalized fluorescence $\left(\Delta \mathrm{F}_{\text {norm }}\right)$ is determined through the ratio of $F_{\text {hot }} / F_{\text {cold }}$ where $F_{\text {hot }}$ is the fluorescence within the red area and $F_{\text {cold }}$ is the fluorescence within the blue area.

The four proteins being measured retain similar sequence composition, where monomeric $\alpha$-synuclein differs from its A53T mutant form by a single missense mutation at the $53^{\text {rd }}$ position along its sequence. Both $\beta$ - and $\gamma$-synuclein are missing segments within the NAC and C-terminal domains, with $\gamma$-synuclein being impacted to a greater extent. For both monomeric and A53T $\alpha$-synuclein, increasing protein concentrations result in decreased fluorescence intensity and ellicit positive thermophoresis, signifying specific aptamer-protein binding. ${ }^{203}$ When examining $\beta$-synuclein, an opposing trend is observed where increasing protein concentrations leads to increased fluorescence and negative thermophoresis. ${ }^{204}$ Positive thermophoresis is more common as a net movement from a 
region of hotter temperature to a region of cooler temperature while negative thermophoresis could be due to solute properties during ASYN2-protein complexation. ${ }^{205}$ It is also possible that a specific region of the protein could intercalate within the aptamer sequence or adopt a conformation that induces aggregation; both resulting in negative thermophoresis.

The binding affinities of ASYN2 for the four proteins was not determined as a plateau was not reached at higher protein concentrations $(>10 \mu \mathrm{M})$. Analyzing at $\alpha$ synuclein concentrations in excess of $10 \mu \mathrm{M}$ is not practical as it is expensive to acquire that volume of lyophilized protein and does not resemble physiological concentrations that would be exhibited within PD patients. $K_{d}$ values for the successful interaction of ASYN2 with monomeric and A53T mutant $\alpha$-synuclein were estimated to be greater than $5.6 \mu \mathrm{M}$ $(\mathrm{N}=2)$ respectively. This binding dissociation is several orders of magnitude higher than what was achieved in EIS analysis, but further illustrates the impact of SELEX conditions on ASYN(1-5) as high binding affinity was a secondary goal of their selection.

With respect to $\beta$-synuclein the MST trace indicates minor binding with ASYN2 had occurred, suggesting a $\mathrm{K}_{d}$ in excess of $10 \mu \mathrm{M}$. This aligns with previously performed EIS characterization tests as ASYN2 was not specific for $\beta$-synuclein due to a lack of sequence homology with monomeric $\alpha$-synuclein. Lack of binding, or presence of nonspecific binding, is possible as the conformation of $\beta$-synuclein is missing a segment within the monomeric NAC domain along with extensive AA substitutions throughout its C-terminal domain. $\gamma$-synuclein is missing majority of the $\mathrm{C}$-terminal domain present in monomeric $\alpha$-synuclein, and similar to EIS studies, was not predicted to possess any binding with ASYN2. 


\subsection{Circular dichroism (CD) of ASYN(1-5)}

CD spectroscopy was performed to determine whether the aptamer sequences had the ability to adopt a G-quadruplex conformation and to explore any target-induced structural changes that occur as a result of target-aptamer complexation. G-quadruplex formation among selected ASYN aptamers (1-5) in BFR-1 was compared and illustrated in Figure 3.26.

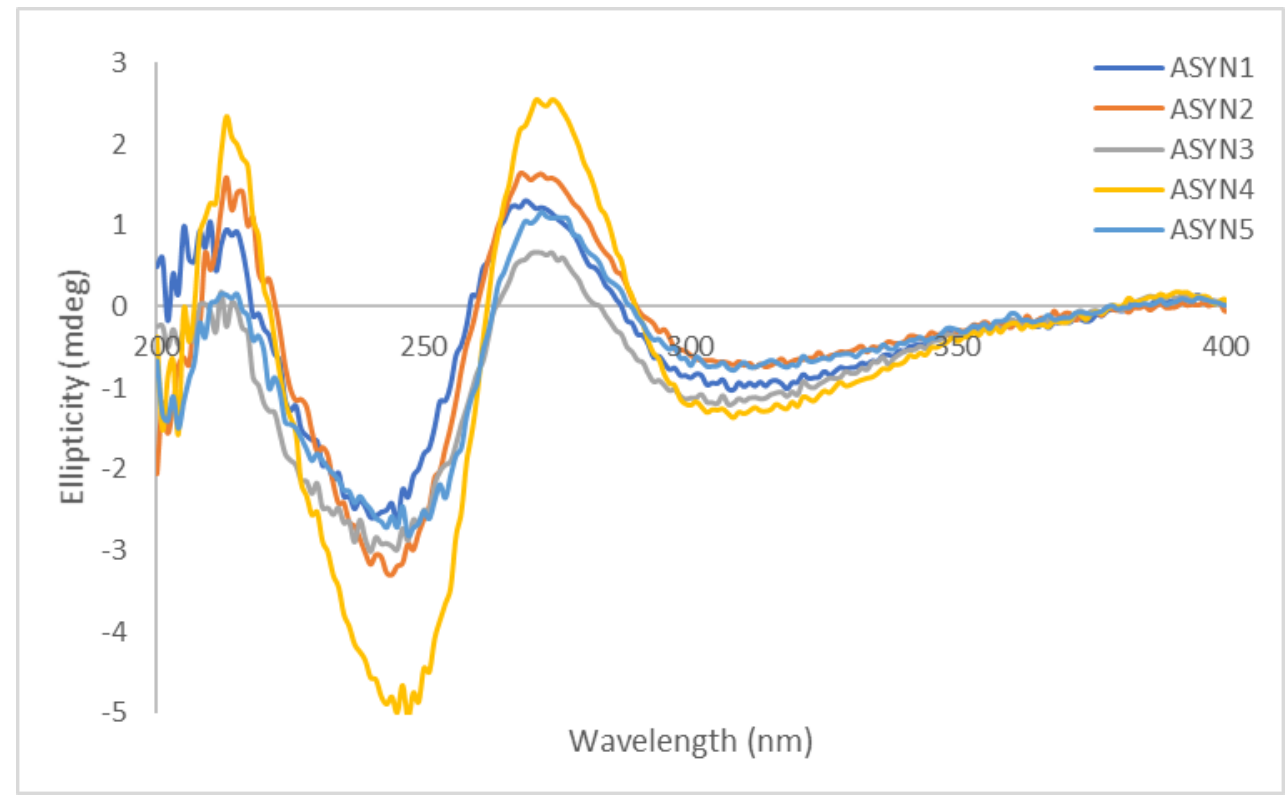

Figure 3. 26: CD spectra of ASYN(1-5) sequences at a concentration of $18.75 \mu \mathrm{M}$ in BFR-1.

All five sequences contain absorption maxima at $216 \mathrm{~nm}$ and $270 \mathrm{~nm}$ with absorption minima at $240 \mathrm{~nm}$ respectively. The presence of both positive peaks at $216 \mathrm{~nm}$ and $270 \mathrm{~nm}$ suggests the presence of a unimolecular parallel G-quadruplex motif. Parallel G-quadruplexes can be distinguished from antiparallel conformations as they contain a positive maximum at $295 \mathrm{~nm}$ of greater intensity and characteristic minimum at 265 nm. ${ }^{138,206}$ Within the parallel G-quadruplex motif of the ASYN sequences, two stacked G- 
tetrads adopt an anti- conformation, where both anti- and syn-orientations of guanine are shown in Figure 3.27.
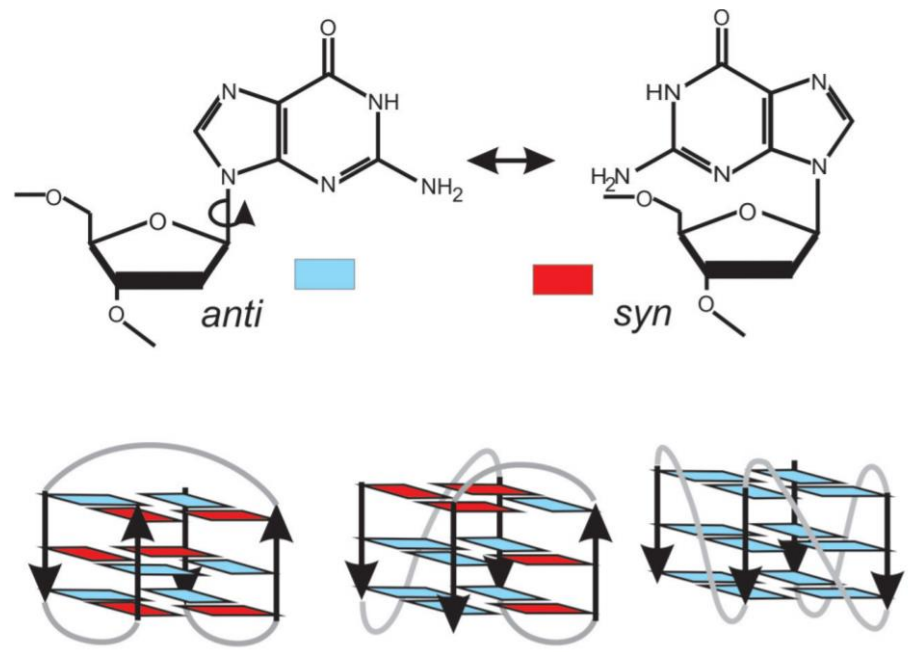

Figure 3. 27: Illustration of respective (A) anti- and (B) syn- guanine orientations within a G-tetrad. The ratio of syn-/anti-guanine within an aptamer dictates the parallel or antiparallel nature of the corresponding G-quadruplex. ${ }^{207}$

A G-quadruplex structure comprised of syn- guanine residues has also been found ${ }^{208}$ containing a small positive $295 \mathrm{~nm}$ peak similar to parallel anti-quadruplexes. The intensity of this $295 \mathrm{~nm}$ peak is typically determined by the syn-/anti- ratio comprising the G-quadruplex where an antiparallel conformation will have a value of $1.0 .^{206,207}$ With respect to ASYN(1-5), a positive shoulder is apparent at the $295 \mathrm{~nm}$ position, supporting the idea that a unimolecular or bimolecular motif with external loops exists as opposed to a quadruplex consisting of a tetramolecular complex. ${ }^{203,206}$ The corresponding CD spectra also indicates the presence of B-form DNA from the positive $216 \mathrm{~nm}$ maximum as A-form DNA depicts an inverse CD with an intense minimum at $216 \mathrm{~nm} .{ }^{138}$ A positive peak at 216 nm for ASYN5, and for ASYN sequences capable of forming a G-quadruplex, could also 
increase in intensity due to the presence of unpaired bases adopting a random coil structure. ${ }^{60}$

The binding of ASYN2 with monomeric $\alpha$-synuclein was examined in BFR-1 over an aptamer:target ratio of 1:1 - 10:1 (Figure 3.28). Conformational changes within ASYN2 in the presence of increasing concentrations of monomeric $\alpha$-synuclein lead to a corresponding peak shift and decrease in peak intensity.

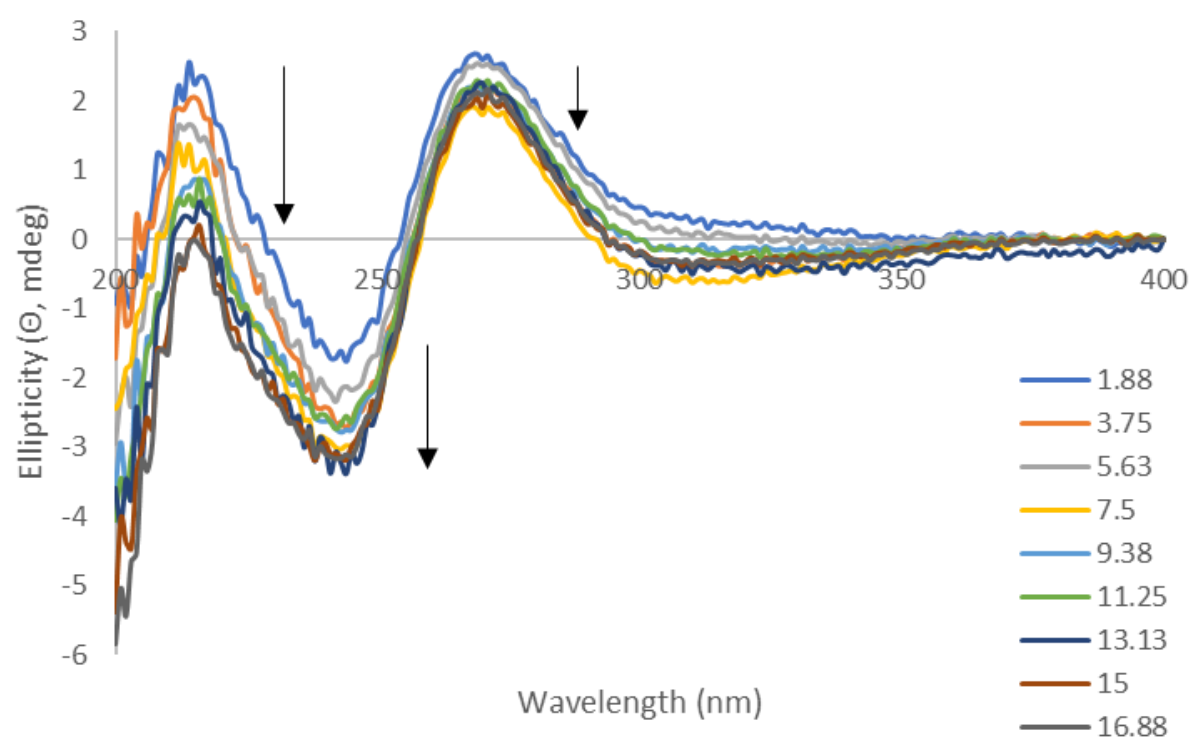

Figure 3. 28: CD spectra of ASYN2 in BFR-1 illustrating the conformational changes within the aptamer sequence as the concentration of monomeric $\alpha$-synuclein is increased from $(0.19 \mu \mathrm{M}-1.875$ $\mu M)$. The arrows indicate the directional change of the ASYN2 peaks.

A small red shift occurs at the $216 \mathrm{~nm}$ peak to $214 \mathrm{~nm}$ at higher target concentrations while the respective intensities of the two positive peaks at $216 \mathrm{~nm}$ and 270 $\mathrm{nm}$ and the negative peak at $246 \mathrm{~nm}$ shift to a more negative ellipticity. A similar trend has been seen in literature $\mathrm{CD}$ spectra that suggests the lengthening and reorientation of a stemloop region to more efficiently interact with its target. ${ }^{60,209}$ Plotting the ellipticity of ASYN2 at $216 \mathrm{~nm}$ in the presence of monomeric $\alpha$-synuclein over a concentration range of $1.85 \mu \mathrm{M}$ to $16.88 \mu \mathrm{M}$ yields a binding curve with a linear dynamic range from $3.75 \mu \mathrm{M}$ 
to $15.00 \mu \mathrm{M}$ (Figure 3.29). A $\mathrm{K}_{\mathrm{d}}$ value of approximately $5.7 \pm 0.1 \mu \mathrm{M}$ was determined in Sigmaplot for ASYN2 by normalizing CD data at the positive ellipticity $(216 \mathrm{~nm})$, consistent with results obtained through MST analysis in Figure 3.25. This approximate $\mathrm{K}_{\mathrm{d}}$ value is in line with what is expected by SELEX as initial sequence selection involved higher protein concentrations and free aptamer in solution.

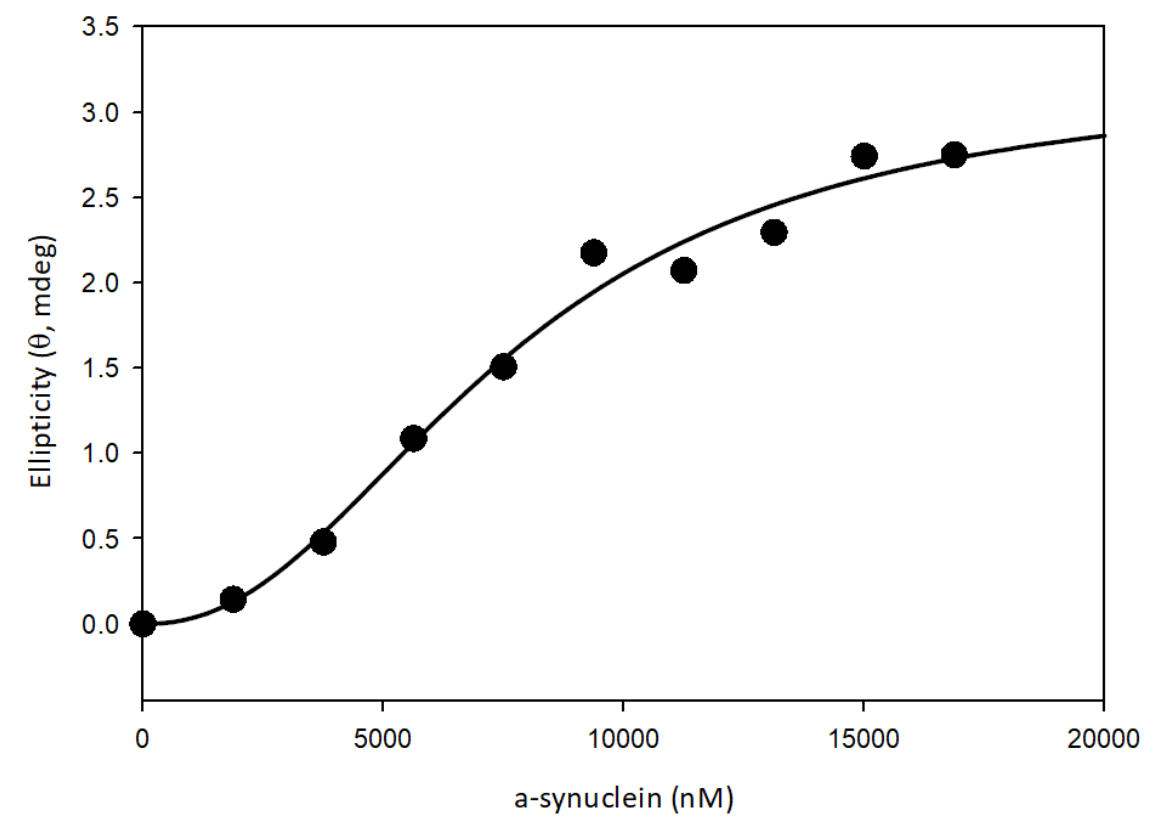

Figure 3. 29: A calibration curve illustrating the CD conformational changes at $216 \mathrm{~nm}$ between ASYN2 and monomeric $\alpha$-synuclein. Sigmaplot was used to determine a $K_{d}$ value of approximately 5 $\mu \mathrm{M}$ for ASYN2.

\subsection{Principle of monomeric $\alpha$-synuclein colorimetric assay}

Gold nanoparticle (AuNP) colorimetric assays were performed as another method of analyzing the binding affinity between ASYN(1-5) and monomeric $\alpha$-synuclein. The AuNPs synthesized for this biosensing system were $15 \mathrm{~nm}$ in diameter and appear as a wine-red colloidal solution due to their electrostatic stabilization (Figure 3.30). The synthesized AuNPs contain negatively charged citrate along its surface, enabling the AuNPs to be well-dispersed in solution. Using UV-Vis spectroscopy, an AuNP plasmon 
absorbance maximum at $\lambda_{\max }=525 \mathrm{~nm}$ and extinction coefficient of $2.7 \times 10^{8} \mathrm{M}^{-1} \mathrm{~cm}^{-1}$ were able to determine the concentration of synthesized AuNPs. ${ }^{210}$ The obtained AuNP concentration of $11.3 \mathrm{nM}$ was similar to literature yields and was enough to conduct this assay.

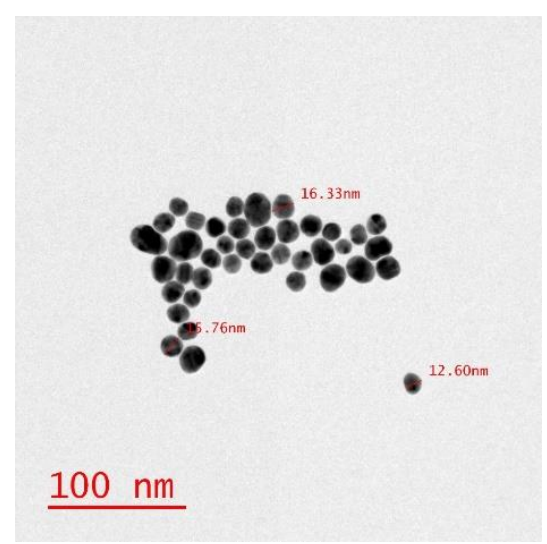

Figure 3. 30: TEM image of synthesized citrate capped AuNPs. The AuNPs appeared as a wine-red solution with an average diameter of $15 \mathrm{~nm}$.

The colorimetric mechanism towards monomeric $\alpha$-synuclein is illustrated in Figure 3.31 as a function of the AuNPs aggregation tendencies. In the absence of DNA and target (Figure 3.31A), citrate-stabilized AuNPs exposed to high concentrations of $\mathrm{NaCl}$ will undergo salt-induced aggregation. ${ }^{211}$ AuNP aggregation is accompanied by a physical colour change from wine-red to a deep-blue, and through a blue shift in UV-vis spectra from $\lambda_{\max }=525 \mathrm{~nm}$ to $\lambda_{\max }=625 \mathrm{~nm}$. In the presence of ssDNA (Figure 3.31B), non-specific interactions between the base ring nitrogen allow for electrostatic and hydrophobic adsorption of the ssDNA to the AuNP surface. Electronegative charge along the DNA backbone inhibits AuNP aggregation in the presence of salt due to surface repulsion among the colloidal AuNPs. The addition of monomeric $\alpha$-synuclein to the mixture causes surface adsorbed DNA eliciting a random coiled structure to undergo a conformational change into a G-quadruplex (Figure 3.31C). Formation of this target- 
induced secondary structure releases ssDNA from the AuNP surface due to the rigid structures inability to adsorb. Loss of surface-bound DNA removes AuNP protection from salt-induced aggregation, and the resulting AuNPs aggregate and turn blue as a result. An additional control that could be performed would be to investigate the effect of monomeric $\alpha$-synuclein on AuNP salt-induced aggregation to determine if there are any inhibitory effects observed.

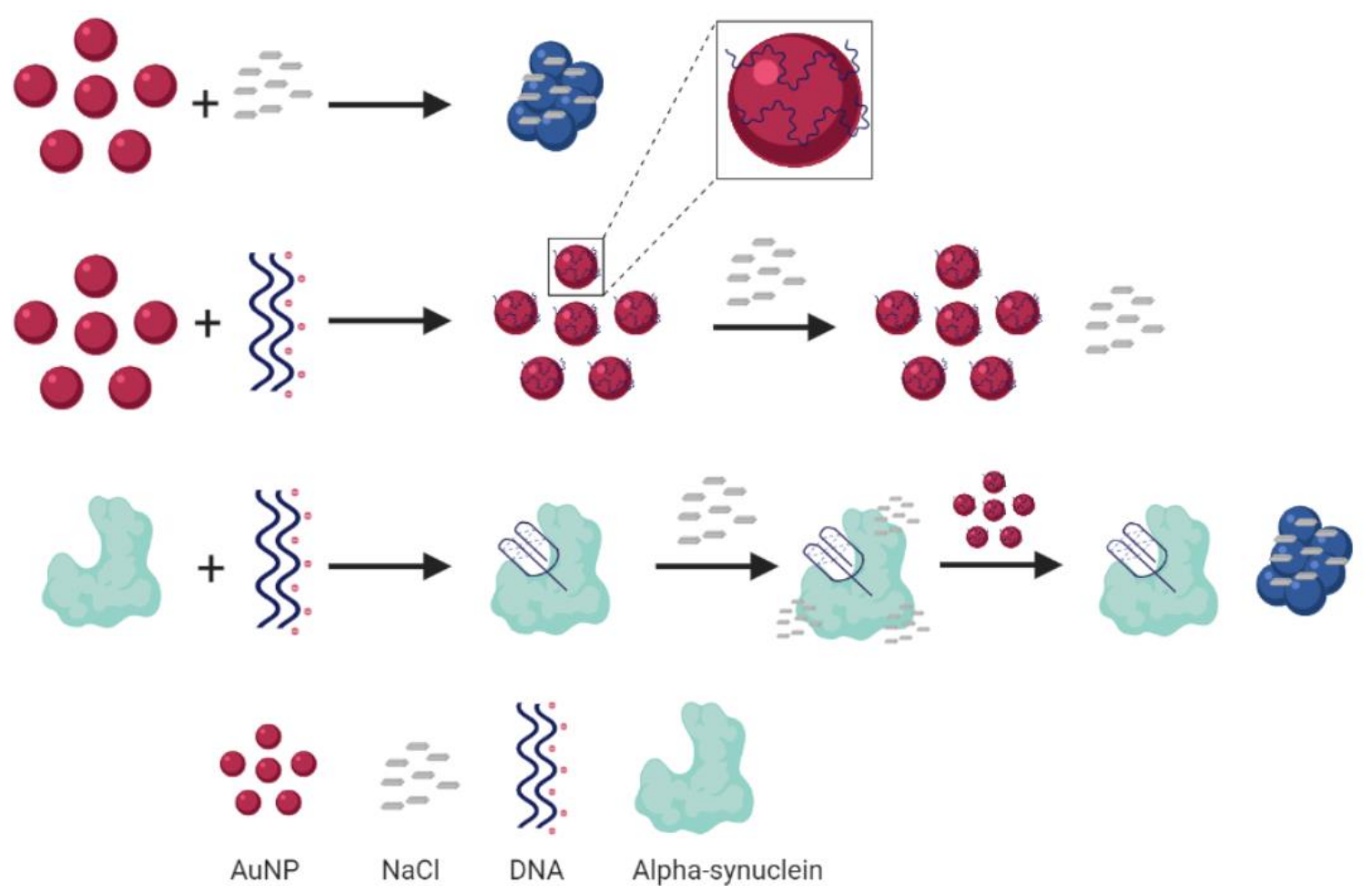

Figure 3. 31: AuNP colorimetric mechanism depicting optical biosensor fabrication and monomeric $\alpha$ synuclein detection. Apt: aptamer; NaCl: sodium chloride; AuNP: gold nanoparticle, $\alpha$-syn: monomeric $\alpha$-synuclein.

\subsubsection{Optimization of colorimetric assay conditions}

The AuNP, salt, DNA, and target concentrations were optimized for the fabrication of the colorimetric biosensor. As shown in Figure 3.32, various concentrations of AuNPs $(0.75 \mathrm{nM}-7.53 \mathrm{nM})$ were prepared from an AuNP stock of $11.0 \mathrm{nM}$ in deionized water 
$\left(\mathrm{DiH}_{2} \mathrm{O}\right)$. With the total sample volume being $150 \mu \mathrm{L}$ an AuNP concentration of $3.77 \mathrm{nM}$ was selected as the optimal concentration. A concentration of $3.77 \mathrm{nM}$ yielded a vibrant red solution without using a high volume of AuNPs as in the $7.53 \mathrm{nM}$ solution, and UVVis spectra indicated that the absorbance at $525 \mathrm{~nm}$ was significantly higher when compared to the minimal absorbance at $625 \mathrm{~nm}$. The high absorption at $525 \mathrm{~nm}$ will allow a broader range of target concentrations to be tested.
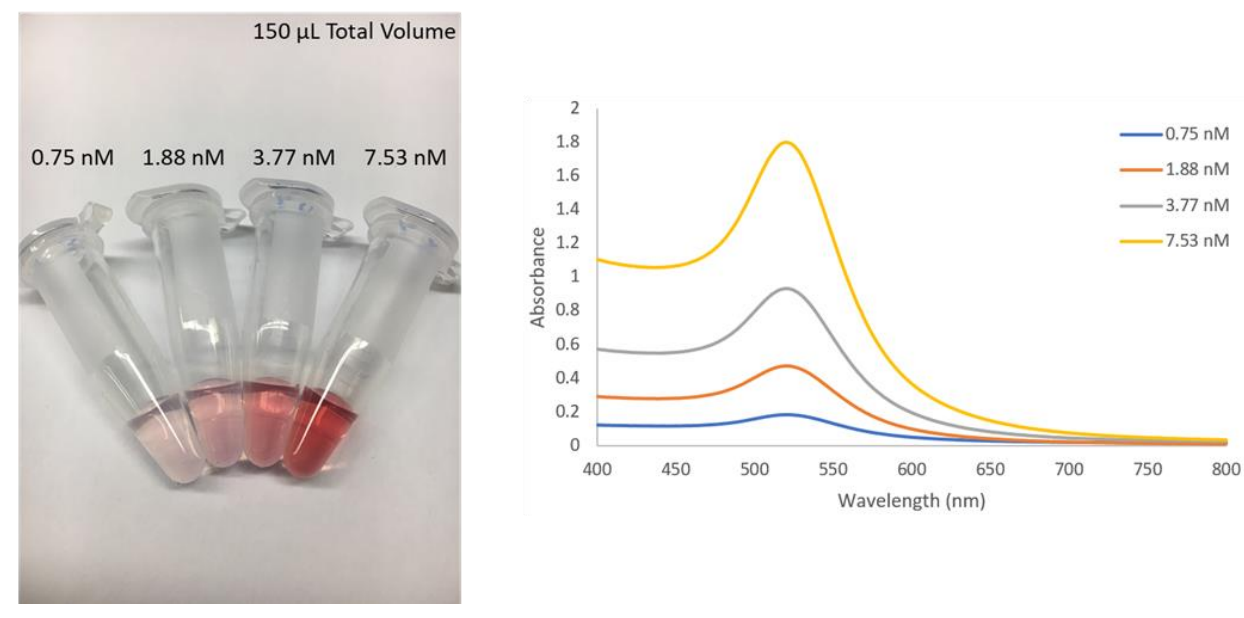

Figure 3. 32: (A) Optimization of AuNP concentration ( $0.75 \mathrm{nM}$ - $7.53 \mathrm{nM}$ ) for colorimetric assays. (B) UV-Vis spectra of the corresponding AuNP concentrations where AuNP concentrations of $3.77 \mathrm{nM}$ were applied for further colorimetric assay analysis.

The concentration of $\mathrm{NaCl}$ necessary for complete AuNP aggregation was optimized in Figure 3.33A using $\mathrm{NaCl}$ concentrations ranging from $0.25 \mathrm{M}$ to $0.5 \mathrm{M}$. Four concentrations of AuNPs $(0.75 \mathrm{nM}-7.53 \mathrm{nM})$ in the presence of $30 \mu \mathrm{L}$ of each $\mathrm{NaCl}$ concentration were compared to determine how the AuNPs aggregate in the respective solutions. The efficiency of AuNP aggregation is illustrated in Figure 3.33B where a UVVis spectra indicates a maximum absorbance at $625 \mathrm{~nm}$ in the presence of $0.4 \mathrm{M} \mathrm{NaCl}$. Figure 3.33C confirms $0.4 \mathrm{M} \mathrm{NaCl}$ is necessary for complete AuNP aggregation where the absorbance ratio at $\mathrm{A}_{625} / \mathrm{A}_{525}$ are compared. A higher $\mathrm{A}_{625} / \mathrm{A}_{525}$ value represents 
aggregation (solution is blue), and a lower $\mathrm{A}_{625} / \mathrm{A}_{525}$ value represents AuNP distribution (solution is red). An increase in aggregation occurs from $0.25 \mathrm{M}$ to $0.4 \mathrm{M} \mathrm{NaCl}$ before plateauing from $0.4 \mathrm{M}$ to $0.5 \mathrm{M} \mathrm{NaCl}$.
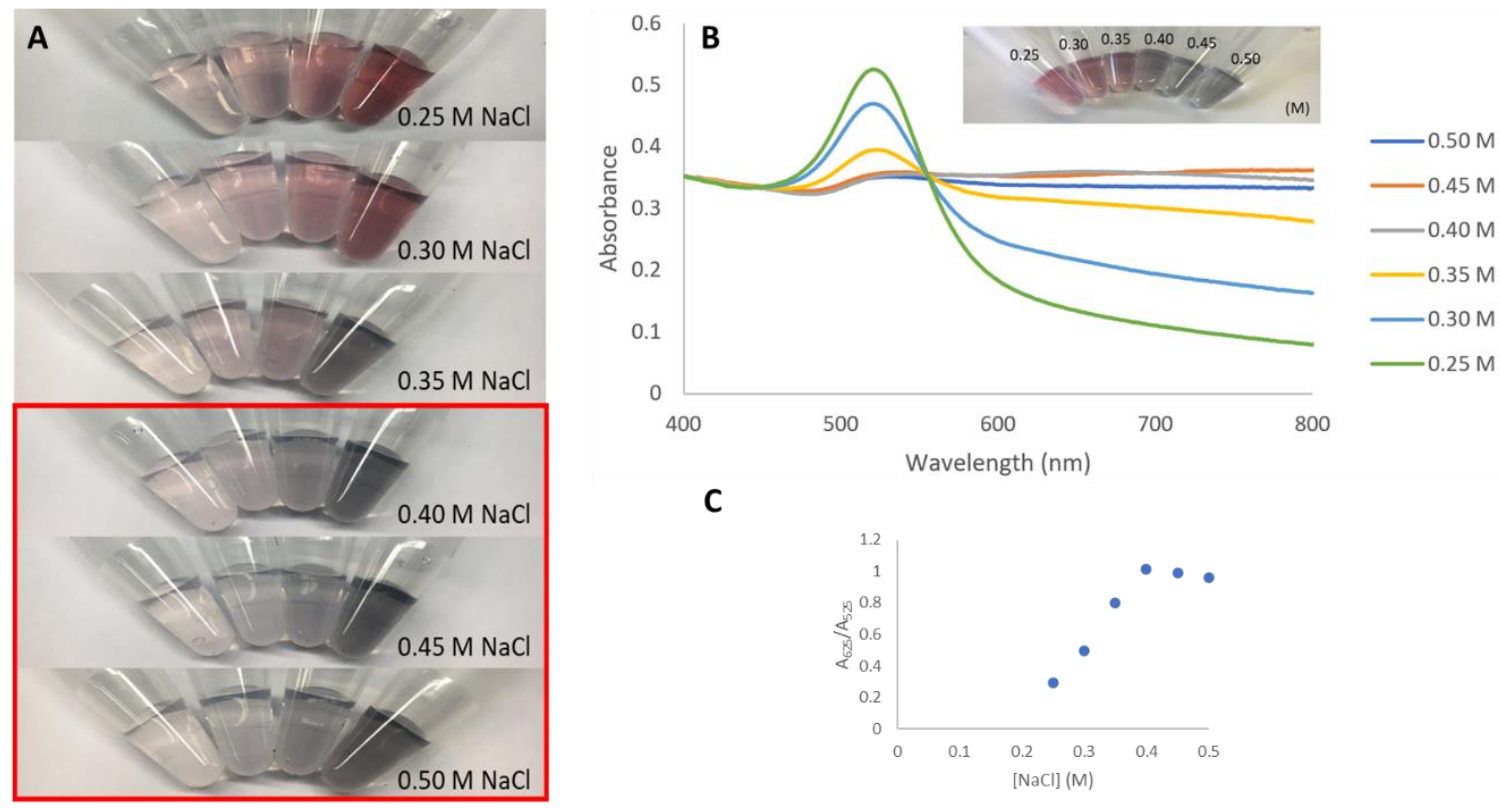

Figure 3. 33: (A) Optimization of $\mathrm{NaCl}$ stock concentration $(0.25 \mathrm{M}-0.50 \mathrm{M})$ for colorimetric assays in the presence of $0.75 \mathrm{nM}-7.53 \mathrm{nM}$ AuNPs. (B) $\mathrm{UV}$-Vis spectra of respective $\mathrm{NaCl}$ stock concentrations $(0.25 \mathrm{M}-0.50 \mathrm{M})$ in the presence of $3.77 \mathrm{nM}$ AuNPs. (C) Plot of the absorbance ratio $\left(\mathrm{A}_{625} / \mathrm{A}_{525}\right)$ vs $\mathrm{NaCl}$ stock concentration where a plateau is observed at $0.4 \mathrm{M}$. In each $\mathrm{NaCl}$ measurement $30 \mu \mathrm{L}$ of the respective $\mathrm{NaCl}$ stock were added to the AuNP solution.

Figure 3.34 illustrates $3.77 \mathrm{nM}$ AuNPs in the presence of the $\mathrm{NaCl}$ concentration range. One advantage to using $0.4 \mathrm{M} \mathrm{NaCl}$ is that the aggregated AuNPs undergo an observable color change without crashing out of solution. This allows the colorimetric sensor to be observed for a longer period of time and provides more consistent results when analyzing samples with UV-vis spectrometry. Using the $0.4 \mathrm{M} \mathrm{NaCl}$ stock solution, the ionic concentration in the presence of $3.77 \mathrm{nM}$ AuNPs was measured to determine if there was an optimal value. $\mathrm{NaCl}$ concentrations ranging $0 \mathrm{mM}$ to $80 \mathrm{mM}$ were compared where 
an observable plateau was expected. Due to the relatively linear increase in $\mathrm{A}_{625} / \mathrm{A}_{525}$, the initial concentration of $60 \mathrm{mM}$ was used for subsequent experiments. ${ }^{212}$

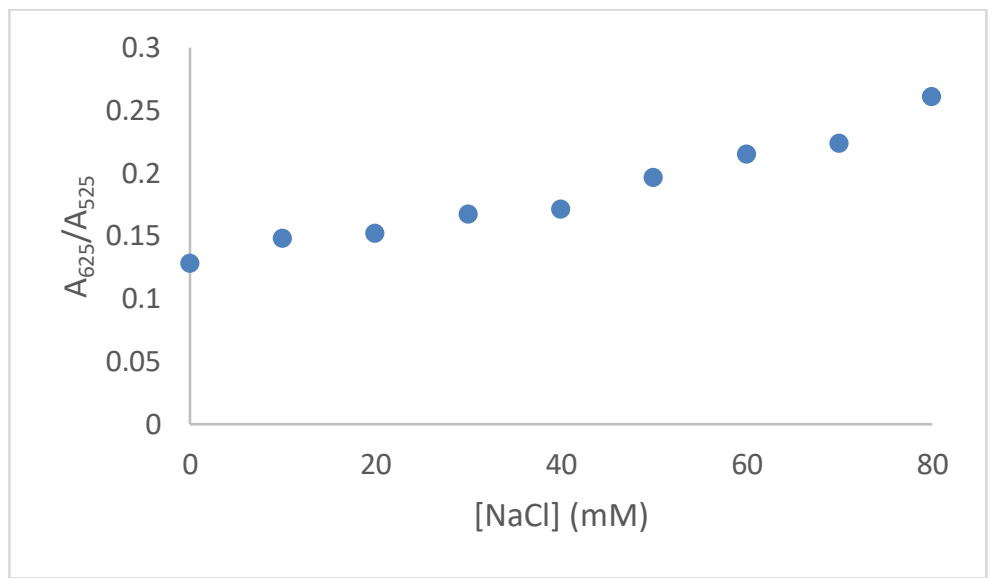

Figure 3. 34: $\mathrm{NaCl}$ concentration range $(0 \mathrm{mM}-80 \mathrm{mM})$ in the presence of $3.77 \mathrm{nM}$ AuNPs. A relatively linear trend was observed, and $60 \mathrm{mM}$ was deemed optimal for the colorimetric assays.

After optimization of $\mathrm{NaCl}$ concentration, optimized DNA can adsorb to AuNP surfaces and prevent salt-induced aggregation. Various concentrations of ASYN aptamer from $0.025 \mu \mathrm{M}$ to $0.35 \mu \mathrm{M}$ were added to $3.77 \mu \mathrm{M}$ AuNPs (Figure 3.35A) for 30 minutes prior to the addition of $60 \mathrm{mM} \mathrm{NaCl}$. Insufficient quantities of DNA directly impacts the stability of AuNPs in solution and would inevitably lead to aggregation. It has been suggested $^{184}$ that DNA has a long Debye length in water, where the Debye length refers to a local electric fields ability to affect the distribution of charge carriers in solution. As the ionic concentration increases, the corresponding Debye length will decrease. ASYN aptamers are long sequences comprised of 66 bases; leading to a greater overall negative charge compared to shorter aptamer sequences. When non-specifically adsorbed to the AuNP surface, the long ASYN strand will encompass a greater surface area along the AuNP than a significantly shorter sequence. Considering DNA has a longer Debye length in $\mathrm{DiH}_{2} \mathrm{O}$ it is possible for electrostatic exclusion zones to form along the surface that repel 
incoming DNA. ${ }^{173,184}$ This would minimize protection of AuNPs upon salt exposure and can be observed in the UV-Vis spectra in Figure 3.35B. The $\mathrm{A}_{625} / \mathrm{A}_{525}$ ratios for the respective concentrations were compared (Figure 3.35C) and a plateau occurred around $0.25 \mu \mathrm{M}$, suggesting that this DNA concentration provided sufficient surface coverage to protect the AuNPs. One study ${ }^{213}$ utilized a terminal polyA tail to further improve the quantity of adsorbed DNA to the AuNP surface. At a neutral $\mathrm{pH}$, adenine becomes protonated due to its $\mathrm{pKa}$ of 3.5 . A pKa value refers to the strength of an acid where a lower pKa indicates a stronger acid that can more readily donate a proton $\left(\mathrm{H}^{+}\right)$. Consecutive adenine bases favours adsorption onto the AuNP surface and effectively tethers the DNA to the surface.
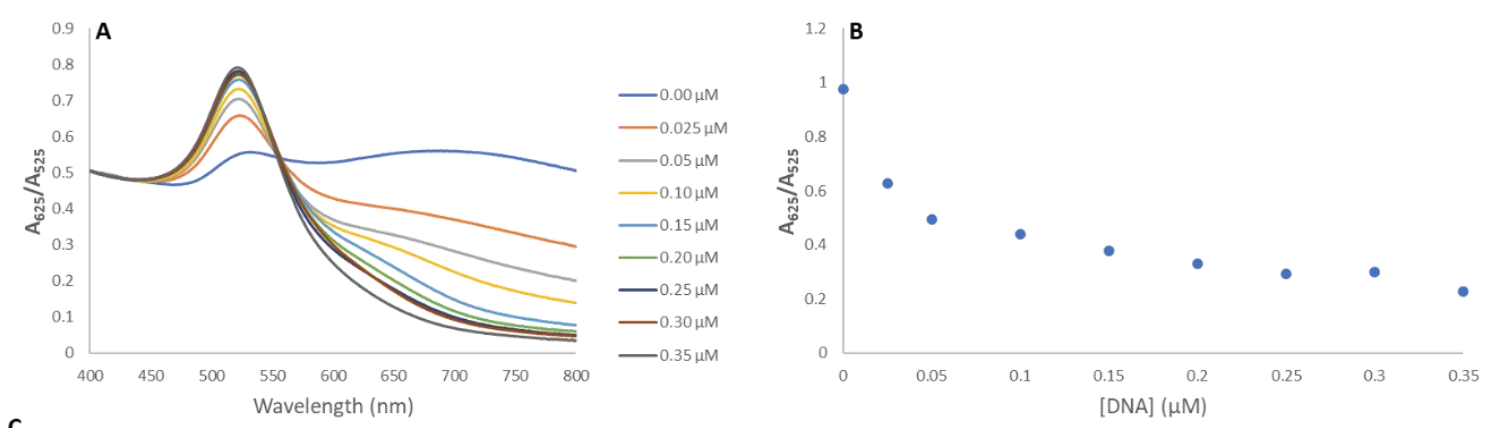

$$
\text { C }
$$
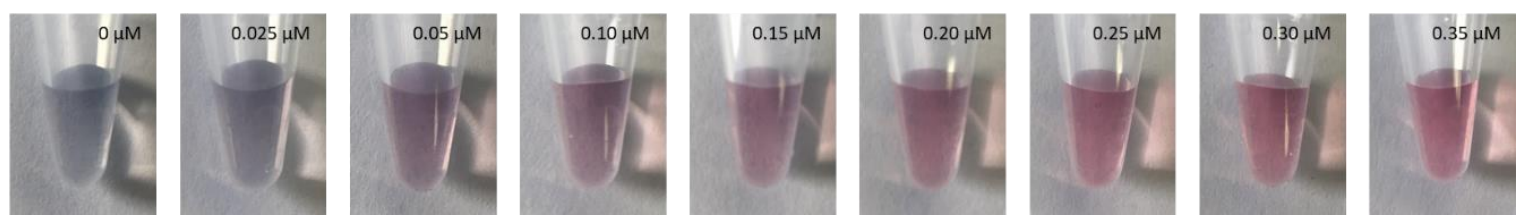

Figure 3. 35: Non-specific DNA interaction with $3.77 \mathrm{nM}$ AuNPs and $60 \mathrm{mM}$ NaCl. (A) UV-Vis spectra of DNA (0.00 $\mu \mathrm{M}-0.35 \mu \mathrm{M})$. (B) Calibration curve displaying the absorbance ratio (A625/A525) vs DNA $(0.00 \mu \mathrm{M}-0.35 \mu \mathrm{M})$ where a plateau occurred at $0.25 \mu \mathrm{M}$. (C) Visual colour change of the AuNPs coated in various concentrations of DNA $(0.00 \mu \mathrm{M}-0.35 \mu \mathrm{M})$.

Monomeric $\alpha$-synuclein concentrations were optimized to determine a working range for binding of ASYN to its protein target. Figure 3.36A depicts various concentrations of $\alpha$-synuclein $(0 \mu \mathrm{M}$ to $1 \mu \mathrm{M})$ in the presence of $3.77 \mathrm{nM}$ AuNPs to confirm 
that the protein does not induce aggregation over the previously examined target range. Interestingly, upon addition of $60 \mathrm{mM} \mathrm{NaCl}$ (Figure 3.36B), protein concentrations above $0.1 \mu \mathrm{M}$ protect AuNPs against aggregation. This suggests an underlying mechanism of $\alpha$ synuclein interaction with the AuNPs that prevents $\mathrm{NaCl}$ from effectively influencing the gold surface. In an effort to make this binding assay compatible with the protein, an alternative approach was required.
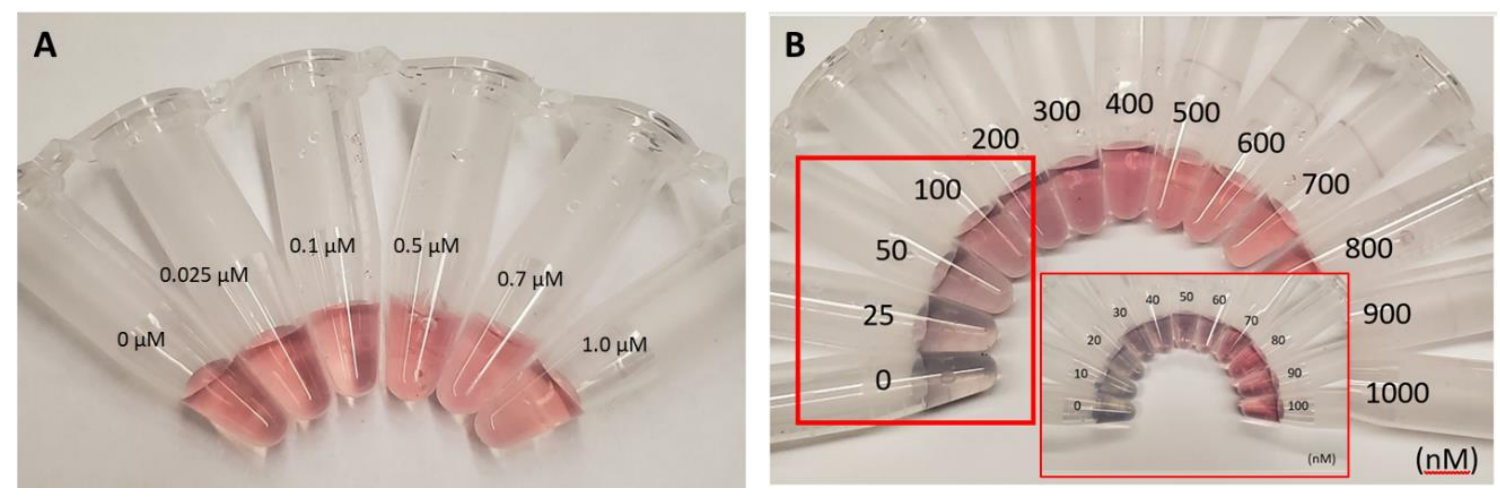

Figure 3. 36: (A) Monomeric $\alpha$-synuclein (0 $\mathrm{nM}$ - $1000 \mathrm{nM}$ ) in presence of $3.77 \mathrm{nM}$ AuNPs. Over the selected concentration range, no AuNP aggregation was observed through AuNP-target interactions. (B) Optical colorimetric biosensor in the presence of monomeric $\alpha$-synuclein $(0 \mathrm{nM}-1000 \mathrm{nM})$. It is apparent that $\alpha$-synuclein concentrations exceeding $200 \mathrm{nM}$ protect AuNPs from salt-induced aggregation.

There are two common approaches for conducting a colorimetric assay that differ in the order of addition of reagents: the forward method, where sequential additions of DNA onto AuNPs, target incubation with the DNA-AuNP complex, and ultimately $\mathrm{NaCl}$, and the reverse method where DNA is incubated with its target for a specified period of time, followed by the addition of $\mathrm{AuNPs}$ and finally $\mathrm{NaCl}$ to induce aggregation. It was found that neither forward nor reverse methods were able to counteract the protective properties of $\alpha$-synuclein on AuNP aggregation. As a result, an alternative method utilizing poly(diallyldimethylammonium chloride) (PDDA) was applied in an attempt to circumvent these issues. 
The use of PDDA in colorimetric assays has gained popularity in literature and offers unique properties over traditionally used salt-induced AuNP aggregation. ${ }^{213-220}$ PDDA is a water-soluble cationic polymer that can both control the aggregation of AuNPs, while electrostatically hybridizing with DNA through its negatively charged phosphate backbone. The general colorimetric mechanism for the application of PDDA is displayed in Figure 3.37.

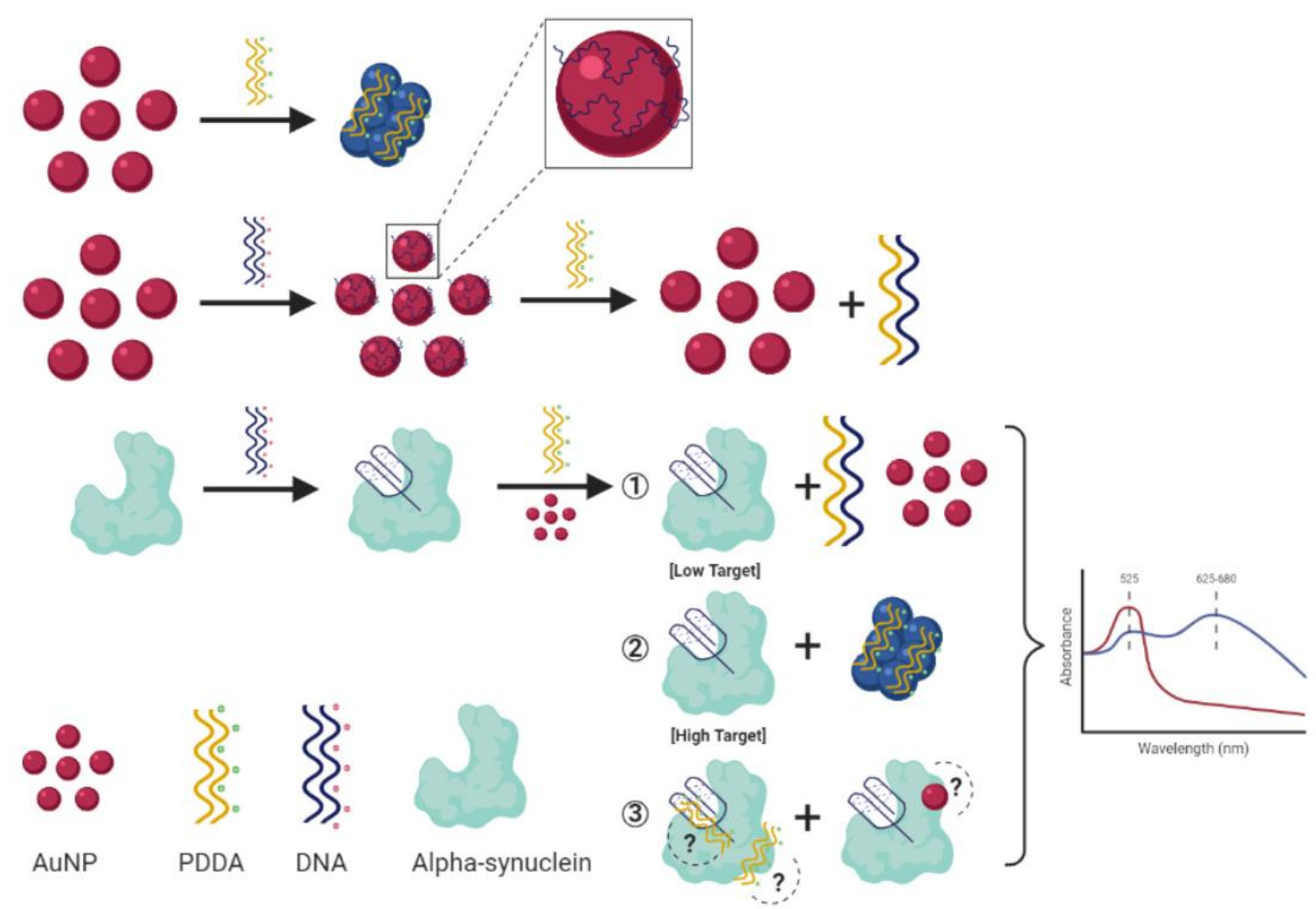

Figure 3. 37: Schematic representation of proposed PDDA colorimetric detection assay. Apt: aptamer; PDDA: poly(diallyldimethylammonium chloride); AuNP: gold nanoparticle; $\alpha$-syn: monomeric $\alpha$ synuclein.

In the absence of monomeric $\alpha$-synuclein, single stranded DNA retains a randomcoil secondary structure. Exposure to PDDA elicits duplex formation with the DNA, leading to an insufficient concentration of PDDA in solution to interact with the exposed AuNPs. As a result, the AuNPs remain stable and well dispersed in solution, yielding a red 
colour. In the presence of monomeric $\alpha$-synuclein, a G-quadruplex conformational change is induced through aptamer-target complexation due to the aptamer's high affinity for the target. The structural change of the aptamer leaves the AuNPs unprotected, allowing remaining PDDA to electrostatically neutralize the citrate capped surfaces of the AuNPs. This will lead to AuNP aggregation, and an observable colour change from red to blue with an accompanying decrease of the SPR peak at $525 \mathrm{~nm}$ for a red-shifted maximum at approximately $680 \mathrm{~nm} \cdot{ }^{213-220}$ The colour of the respective assay directly correlates to the concentration of unbound PDDA in solution following monomeric $\alpha$-synuclein addition.

Figure 3.37 depicts various interactions between the colorimetric assay constituents that that may cause results to deviate from what is anticipated. Binding between PDDA and the aptamer-target complex may consume excess PDDA in solution through interactions with the DNA phosphate backbone or an electronegative region along the target sequence that would minimize AuNP aggregation. Another consideration was the interaction between target and AuNPs, spatially restricting the AuNPs from achieving a close proximity despite the abundance of free PDDA.

Using this new mechanism, the concentration of PDDA necessary to completely aggregate AuNPs was determined (Figure 3.38). Various concentrations of PDDA (3.78$1.89 \times 10^{-7} \mathrm{mM}, 15 \mu \mathrm{L}$ ) were added to a vial containing $85 \mu \mathrm{L}$ of $\mathrm{DiH}_{2} \mathrm{O}$ prior to addition of $50 \mu \mathrm{L}$ of $11.3 \mathrm{nM}$ AuNPs. Visually, the corresponding colour shift was very noticeable where a PDDA concentration of $1.89 \times 10^{-4} \mathrm{mM}$ was necessary for complete AuNP aggregation. UV-Vis spectra of the respective samples were performed where the $\mathrm{A}_{680} / \mathrm{A}_{525}$ ratios were recorded and compared. 


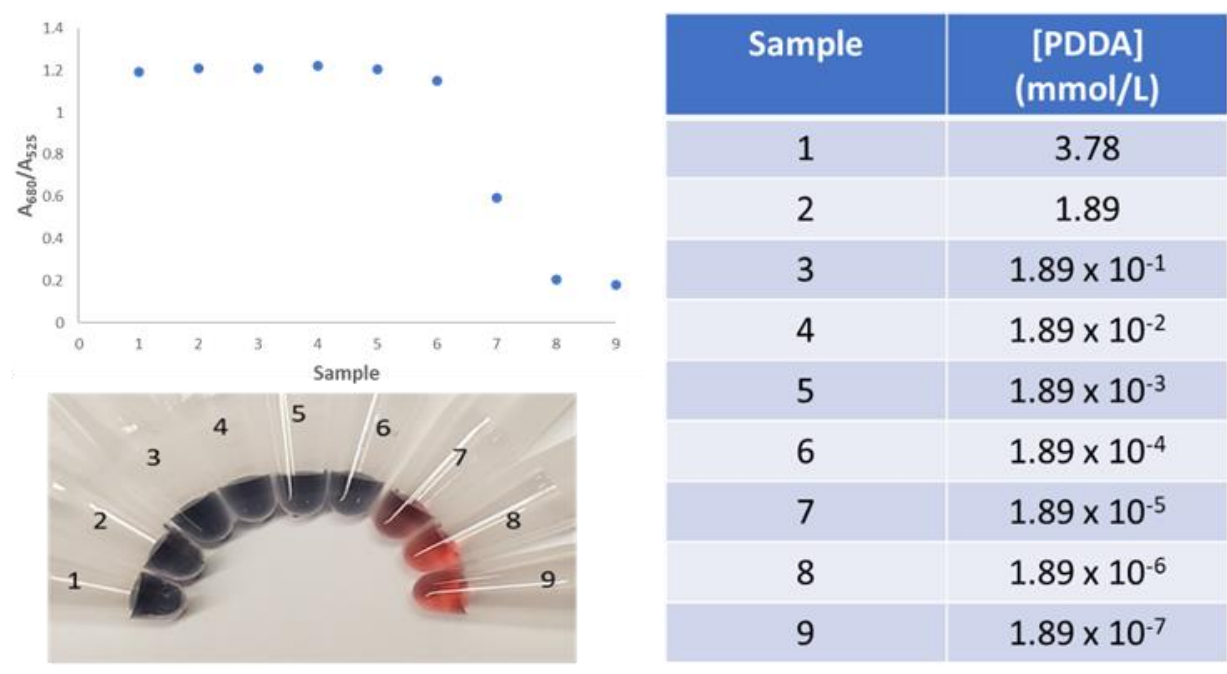

Figure 3. 38: Optimization of PDDA on $3.77 \mathrm{nM}$ AuNPs. A plateau was observed at $1.89 \times 10^{-4} \mathrm{mM}$, proving to be an ideal PDDA concentration for the complete aggregation of AuNPs.

As the concentration of PDDA in solution increased, the respective $\mathrm{A}_{680} / \mathrm{A}_{525}$ ratio also increased until a plateau occurred from $1.89 \times 10^{-4}-3.78 \mathrm{mM}$ PDDA. This further confirmed $1.89 \times 10^{-4} \mathrm{mM}$ PDDA was a sufficient concentration for AuNP aggregation and was used for the remainder of experimentation.

The optimization of ASYN was performed in the presence of $1.89 \times 10^{-4} \mathrm{mM}$ PDDA and $3.77 \mathrm{nM}$ AuNPs. Throughout literature, several papers suggest incubating DNA with AuNPs prior to PDDA addition and others suggest performing the opposite where PDDA is added to DNA prior to AuNP addition. To determine if there is a significant difference between each method, both were optimized. ASYN concentrations were varied $(0 \mu \mathrm{M}$ to $200 \mu \mathrm{M})$ and incubated in solutions containing $15 \mu \mathrm{L}$ of $1.89 \times 10^{-4} \mathrm{mM}$ PDDA and $3.77 \mathrm{nM}$ AuNPs in $\mathrm{DiH}_{2} \mathrm{O}$. After a 30 min incubation period, PDDA or AuNPs were added respectively to the DNA complex. 

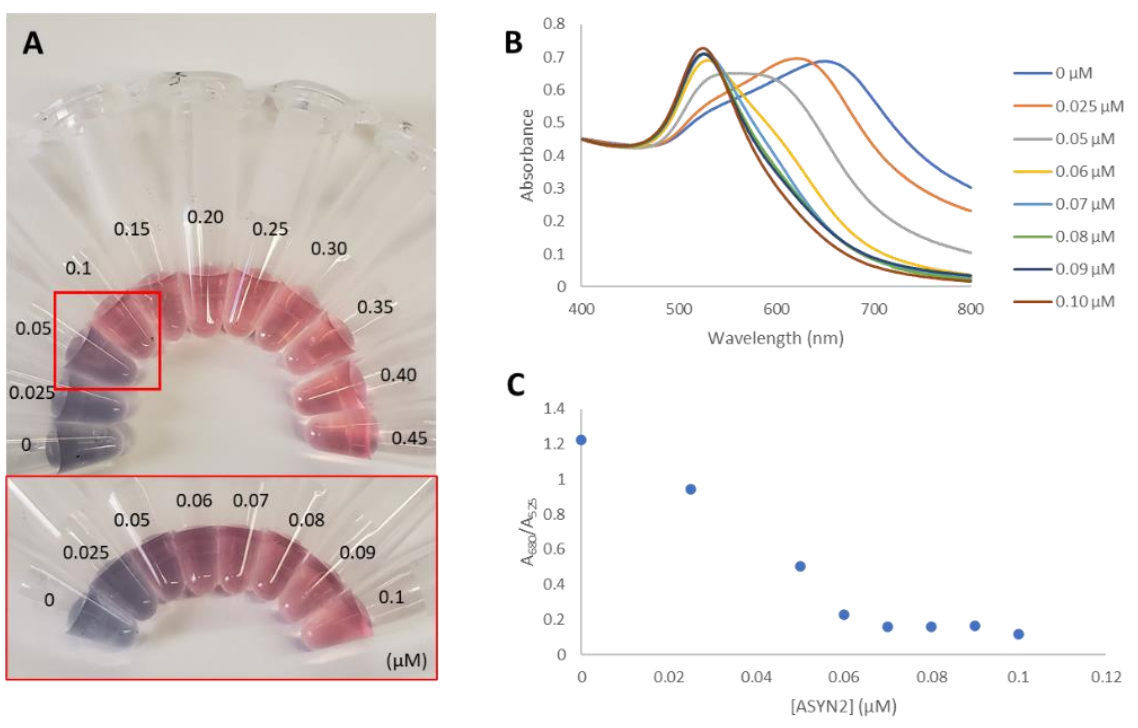

Figure 3. 39: (A) The optimization of ASYN2 was performed using $3.77 \mathrm{nM}$ AuNPs and $1.89 \times 10^{-4} \mathrm{mM}$ PDDA. ASYN2 $(0.00 \mu \mathrm{M}-0.45 \mu \mathrm{M})$ The inset depicts a smaller concentration range $(0.00 \mu \mathrm{M}-0.10$ $\mu M)$. (B) UV-Vis spectra of ASYN2 (0.00 $\mu \mathrm{M}-0.10 \mu \mathrm{M})$. (C) Calibration curve of absorbance (A680/A525) depicts a plateau at $0.07 \mu \mathrm{M}$ ASYN2.

As observed in Figure 3.39, a concentration of 70 nM ASYN proved efficient for AuNP protection from PDDA-induced aggregation in both methods of performing the colorimetric assay. The use of $\mathrm{Mg}^{2+}$ in solution was performed in literature to fabricate a more sensitive colorimetric assay. ${ }^{215} \mathrm{Mg}^{2+}$ enables aptamer-target binding while stabilizing a hairpin secondary conformation, and a concentration of $1 \mathrm{mM} \mathrm{Mg}^{2+}$ during aptamertarget complexation was deemed optimal. ${ }^{215}$

The interactions occurring within the biosensor between DNA-PDDA and DNAtarget were compared to determine how complexation alters aptamer conformation. CD spectroscopy was performed in $\mathrm{DiH}_{2} \mathrm{O}$ to mimic the biosensing conditions of the colorimetric assay (Figure 3.40). 


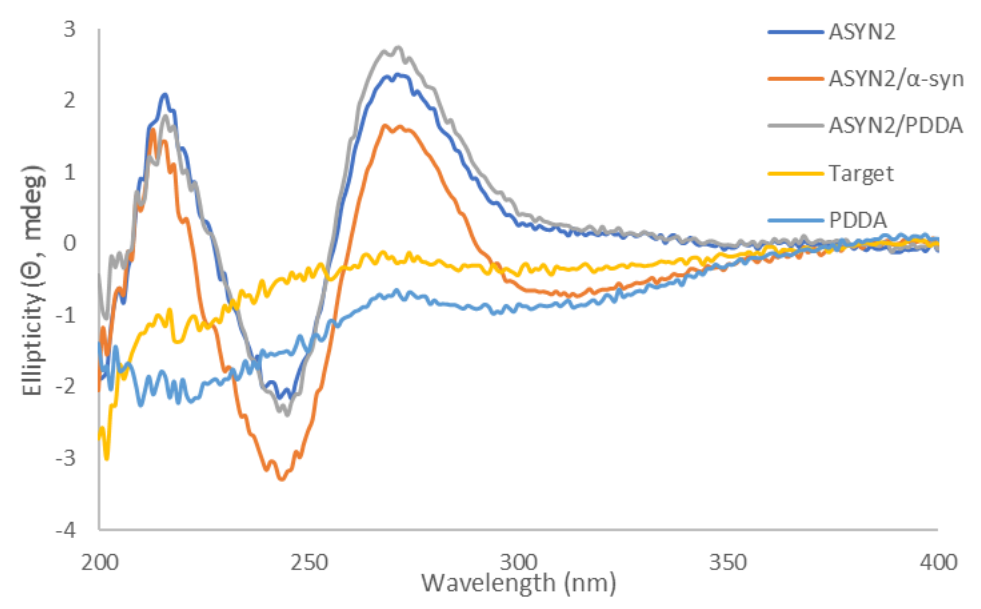

Figure 3. 40: CD spectra of ASYN2 in the presence of monomeric $\alpha$-synuclein (1:1 molar ratio) and PDDA (1.89 $\left.\times 10^{-4} \mathrm{mM}\right)$. It was determined that PDDA does not induce conformational changes upon hybridization with ASYN2.

ASYN2 has been previously determined to contain a centralized G-quadruplex binding motif in PBS. Lack of ionic conditions, specifically an absence of $\mathrm{K}^{+}$ions, could potentially affect ASYN2's ability to adopt a G-quadruplex conformation. It was predicted that PDDA would not significantly alter the DNA secondary structure, enabling DNA to preferentially interact with the target upon exposure. ASYN2 aptamer measurements indicated positive ellipticity peaks in the ranges of $260-280 \mathrm{~nm}$ and $210-220 \mathrm{~nm}$, with negative ellipticity observed between $240-260 \mathrm{~nm}$. These three peaks are characteristic of G-quadruplex formation, suggesting that the aptamer is still capable of forming the desired secondary structure in the absence of alkali metal ions. Hybridization of ASYN2 and PDDA resulted in an increase of the negative peak at 240-260 $\mathrm{nm}$ and decrease of the positive peak at $260-280 \mathrm{~nm}$. It has been suggested ${ }^{218}$ that ASYN2 has maintained its initial structure in $\mathrm{DiH}_{2} \mathrm{O}$, and PDDA has electrostatically bound to the negatively charged phosphate backbone. Interaction between ASYN2 and monomeric $\alpha$-synuclein yielded 
significant decreases in both positive and negatives peaks of the CD spectra, indicating a conformational change as a function of target binding.

A final experimental parameter that was optimized was the incubation time of AuNPs with the ASYN-Target complex. Following both 30 min ASYN-Target and 10 min PDDA incubation periods, AuNPs were added for $1 \mathrm{~min}$ and $30 \mathrm{~min}$ respectively to determine how incubation time affects the biosensor colour change. It was determined that the length of time AuNPs are incubated with the optical biosensor does not affect the corresponding colour change.

\subsubsection{ASYN2 colorimetric assay for monomeric $\alpha$-synuclein}

The optimized colorimetric assay was applied for the detection of monomeric $\alpha$ synuclein and monitored visually, as well as spectroscopically using UV-Vis absorbance values at $\mathrm{A}_{680} / \mathrm{A}_{525}$. Concentrations of target over the range of $0 \mu \mathrm{M}$ to $400 \mu \mathrm{M}$ were tested with their UV-Vis absorbances recorded. Two colorimetric assays were compared where $70 \mathrm{nM}$ ASYN was incubated with a specified concentration of $\alpha$-synuclein, prior to the addition of PDDA before AuNP, or the addition of AuNP followed by PDDA respectively. In Figure 3.41, it is observed that when PDDA is added to the ASYN-Target complex for 10 min before AuNP, the corresponding samples experience varying degrees of aggregation and adopt a blue or purple colour. When AuNPs are added to the ASYN-Target complex for $1 \mathrm{~min}$ prior to PDDA, the samples all remain red with the samples on the lower end of the concentration range exhibiting a darker red colour (A). When examining both trials, visual analysis goes against what is expected where a darker colour (blue) is observed 
at a lower concentration and a lighter colour (red) is observed at higher concentrations of $\alpha$-synuclein (B).
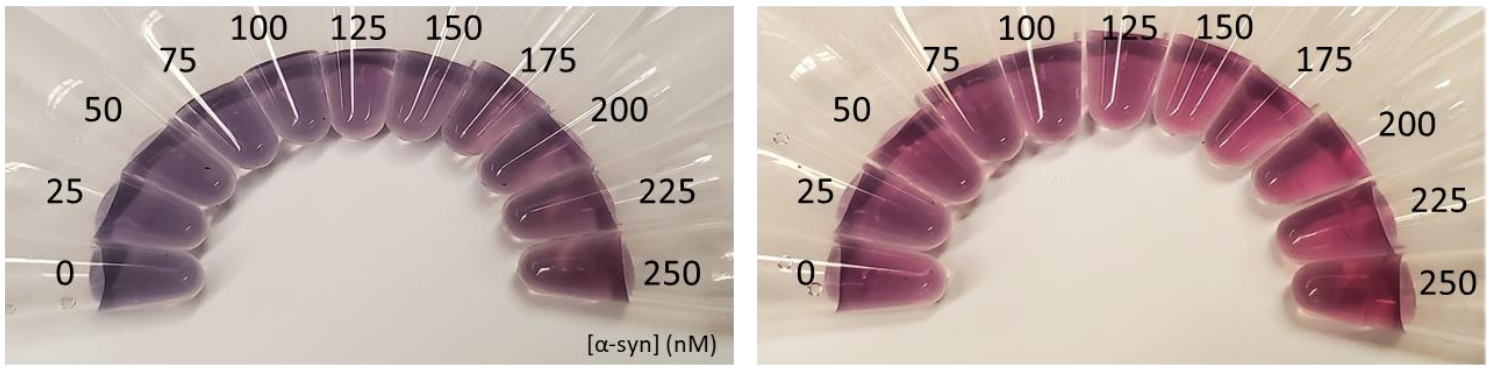

Figure 3. 41: Colorimetric assay using 3.77 nM AuNPs, $70 \mathrm{nM}$ ASYN2, 1.89 x $10^{-4}$ mM PDDA, monomeric $\alpha$-synuclein (0 $\mathrm{nM}$ - $250 \mathrm{nM}$ ). (A) Addition of AuNP/Apt/PDDA/ $\alpha$-syn turned the solution purple across the target concentration range. (B) Addition of AuNP/Apt/a-syn/PDDA kept the solution red across the target concentration range. It was observed in both cases that a darker colour was obtained at lower concentrations, further exhibiting the protective effects of $\alpha$-synuclein on AuNPs at higher concentrations.

Characterization of the aptasensor under various conditions was performed using TEM to determine the state of AuNP aggregation under in the influence of various colorimetric assay parameters. (Figure 3.42) illustrates AuNPs in the presence of ASYN2, PDDA, and monomeric $\alpha$-synuclein target. Synthesized AuNPs were approximately $15 \mathrm{~nm}$ in diameter, spherical and dispersed in solution (A). The physical adsorption of ASYN2 on the AuNP surface caused the AuNPs to become monodispersed in solution due to the electrostatic repulsion of the ASYN2 phosphate backbone (B). Upon addition of PDDA to the bare AuNPs, the cationic polymer neutralized the gold surface and induced aggregation. This aggregation was accompanied by a physical colour change from red to blue (C). When PDDA was added to a solution of physically adsorbed ASYN2 on the AuNP surface, ASYN2 exhausted the concentration of free PDDA in solution. ASYN hybridization prevented PDDA-induced aggregation, and the solution remained red and dispersed (D). Two concentrations of monomeric $\alpha$-synuclein target, $0.25 \mu \mathrm{M}$ and $2.5 \mu \mathrm{M}$, were prepared 
to visualize the unpredicted results discussed previously. At low target concentrations (E), it is clear that significant AuNP aggregation occurred, while at high target concentrations (F) AuNPs appear to be evenly dispersed in solution with minimal aggregation.

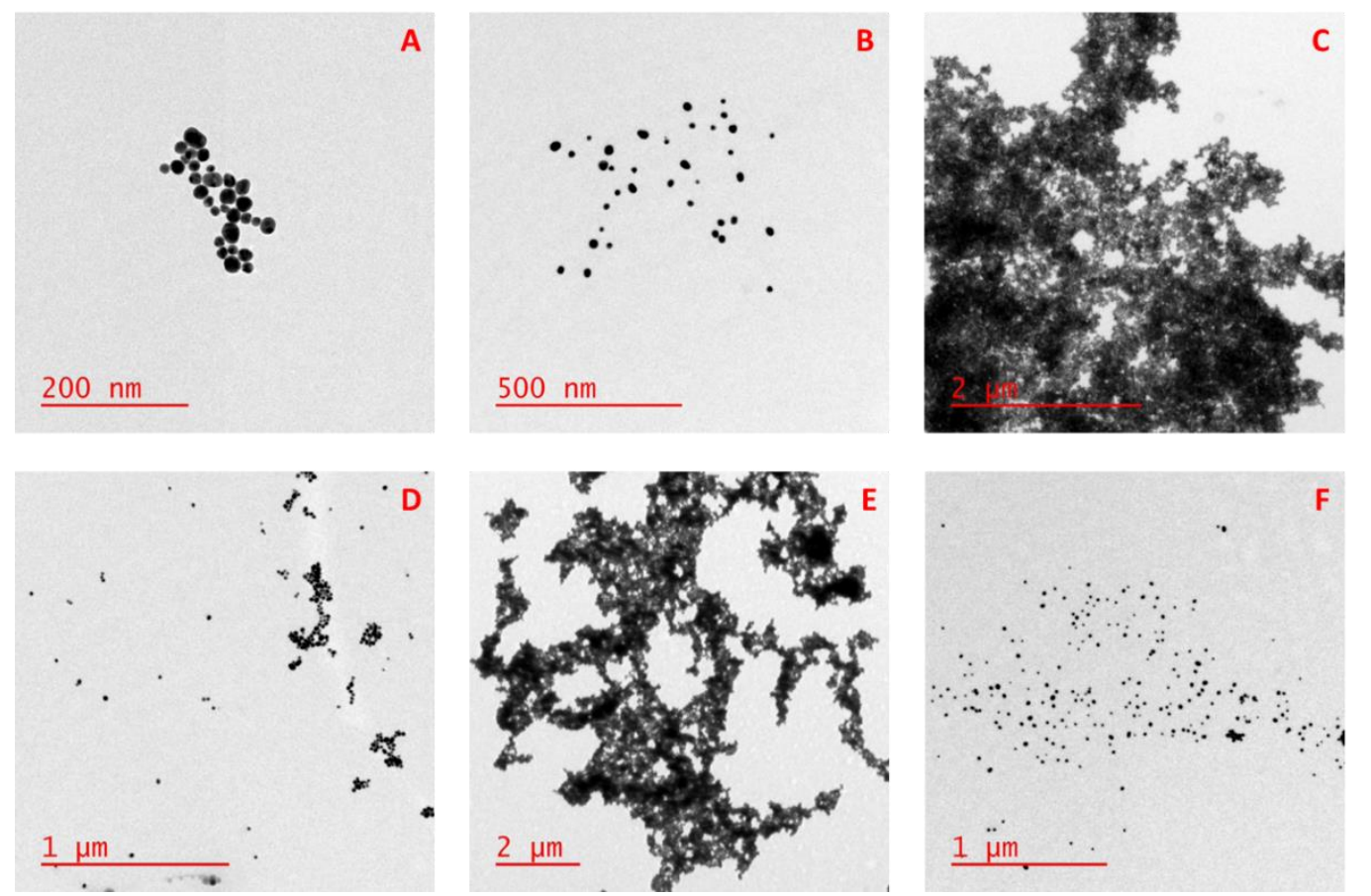

Figure 3. 42: TEM images of AuNP solutions in the presence of varying optimized parameters. (A) 3.77 nM AuNP blank, (B) AuNPs + 70 nM ASYN2, (C) AuNPs + $1.89 \times 10^{-4} \mathrm{mM}$ PDDA, (D) AuNPs + ASYN2 + PDDA, (E) AuNPs + ASYN2 + PDDA + 250 nM $\alpha$-syn, (F) AuNPs + ASYN2 + PDDA + 2500 nM $\alpha$-syn.

There are several potential reasons to explain the opposite trend that is observed in the conducted colorimetric assays. Earlier optimization studies have indicated that AuNPs exposed to increasing concentrations of monomeric $\alpha$-synuclein does not induce aggregation. Therefore, aggregation can be attributed to DNA being removed from the AuNP surface through target complexation or hybridization with PDDA. At lower $\alpha$ synuclein concentrations, it is likely that ASYN is complexing with its protein target through secondary structure conformation changes. This decreases its presence on the AuNP surface through reduced contact points and surface area coverage, allowing PDDA 
to effectively interact with remaining surface adsorbed DNA and exposing the remaining surface to PDDA-induced aggregation. It is also possible for PDDA to be consumed by $\alpha$ synuclein prior to ASYN through interactions with the proteins highly acidic tail domain. This reasoning helps explain the reversed physical observations upon exposure to higher target concentrations. The complete consumption of PDDA by the acidic domain would result in less ASYN-PDDA hybridization, enabling AuNPs to remain dispersed in solution due to ASYN stabilization. PDDA could interact with the target, but not restrict its structural freedom in solution, enabling ASYN to adopt a binding conformation for $\alpha$ synuclein. With both ASYN and PDDA bound to their respective regions within $\alpha$ synuclein, AuNPs would remain stable and well-dispersed due to surface adsorbed citrate.

It has also been hypothesized that the monomeric protein directly interacts with the AuNP surface, mimicking the protection effect of the citrate capped surface and strengthening AuNP stability (Figure 3.43).

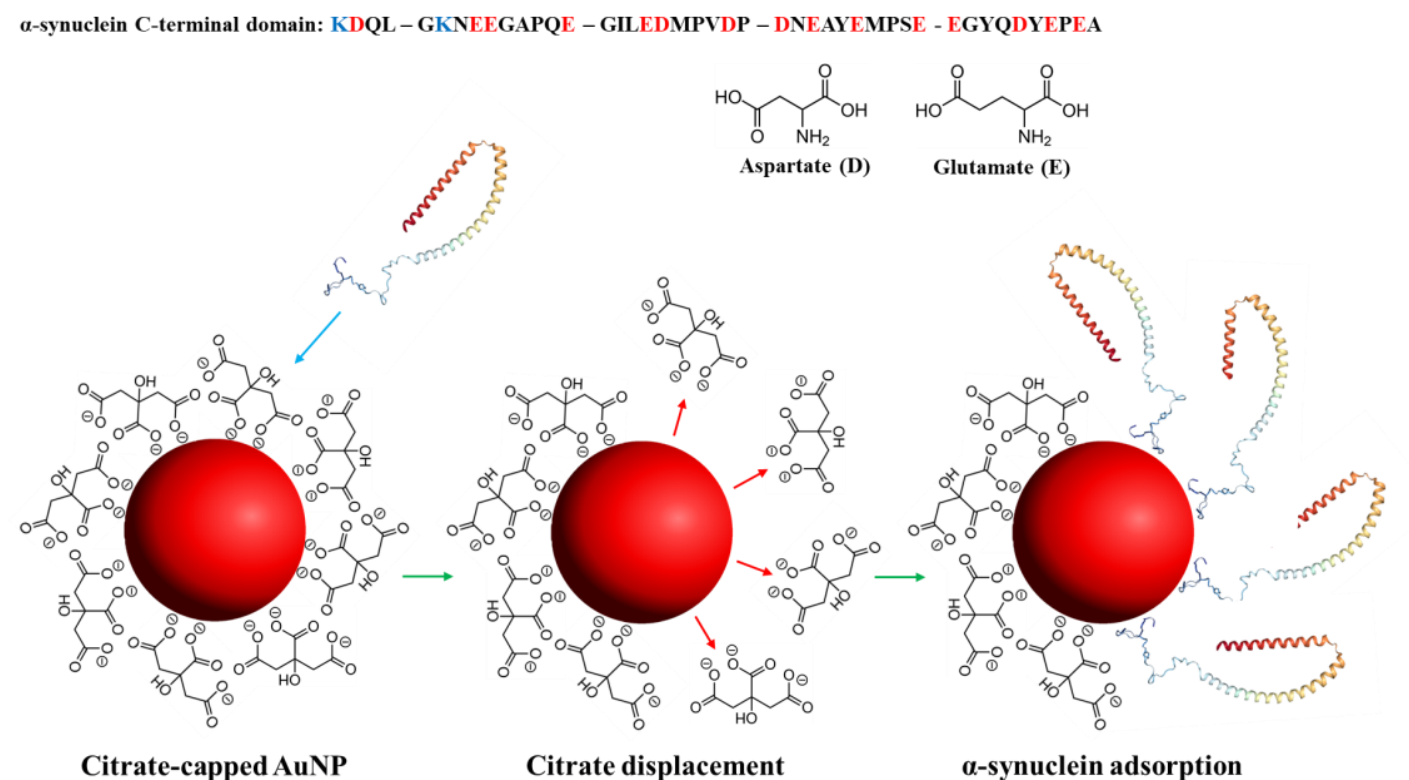

Figure 3. 43 Proposed interaction between monomeric $\alpha$-synuclein acidic C-terminal domain and the AuNP surface through citrate displacment. 
When examining the protein structure of $\alpha$-synuclein, charge patches can be observed throughout with emphasis on the tail region containing 11 acidic residues. The overall surface charge on the protein depends on the $\mathrm{pH}$ of the system it is contained. Within the colorimetric assays, a neutral $\mathrm{pH}$ of 7.4 was applied as this closely resembles physiological conditions and could provide a $\mathrm{K}_{\mathrm{d}}$ value most representative for the aptamers designed application. The isoelectric point $(\mathrm{pI})$ of a protein is the $\mathrm{pH}$ value when the net charge is zero. Decreasing or increasing the $\mathrm{pH}$ of the system with respect to the protein $\mathrm{pI}$ will result in a net positive or negative charge to occur. ${ }^{221}$ The $\mathrm{pI}$ value of $\alpha$-synuclein has been suggested to be $4.7-4.9,{ }^{10,105,142}$ indicating that a $\mathrm{pH}$ of 7.4 will cause the protein to adopt a negative overall charge predominately in its acidic tail domain.

When interacting with an AuNP surface, size is a considerable factor that will facilitate protein-AuNP complexation. With smaller AuNPs, high surface curvatures significantly impact species adsorption to the surface. With the AuNPs used in this study at approximately $15 \mathrm{~nm}$ in diameter, there is sufficient surface area for the protein to develop a stable binding conformation and effectively complex the surface. Several literature studies describe this as protein corona formation, where a kinetic equilibrium is established between a protein and a gold surface dependent on its binding affinity. ${ }^{222-224}$

Two potential methods of complexation can occur between a protein and an AuNP: electrostatic stabilization and citrate displacement. Due to the lack of $\mathrm{NH}_{3}{ }^{+}$containing amino acid residues within $\alpha$-synuclein, it is not likely to associate via this mechanism and surface displacement is a more indicative method. Upon $\alpha$-synuclein addition, the citrate layer is disrupted from the functional groups of select amino acid residues. One study ${ }^{225}$ examined the ability of various amino acids among cysteine (Cys), arginine (Arg), and 
glutamic acid (Glu) to replace citrate within an AuNP surface. It was found that cysteine is the most effective, due to the thermodynamically favourable interaction between its sulfur atom and the gold surface, with glutamic acid being an intermediate amino acid for replacing adsorbed citrate. The two $\mathrm{COO}^{-}$groups of glutamic acid undergo similar interactions with the gold surface as citrate, orienting themselves in this favourable orientation and displacing 5-8 citrate molecules. ${ }^{225}$

The binding of ubiquitin to silver nanoparticles (AgNPs) and its displacement of citrate was also studied. ${ }^{226}$ It was found that at a neutral $\mathrm{pH}$, a negatively charged cluster of AAs within the protein structure interacted stronger with the silver surface than the negatively charged citrates. This suggests that $\alpha$-synuclein electrostatic interactions with the AuNP surface was the driving force instigating protein-AuNP complexation. Steric effects that would be experienced between AuNPs possessing a protein coating would provide greater colloidal stability, as hypothesized in earlier results. Adsorption of $\alpha$ synuclein in a native or denatured state to the AuNP surface will dictate whether ASYN retains affinity for the protein and selectively interact with the designated binding domain. If ASYN binds to $\alpha$-synuclein, excess PDDA may not be able to overcome steric interactions among protein and induce AuNP aggregation. On the other hand, if ASYN is not capable of binding $\alpha$-synuclein, it may hybridize with PDDA where its consumption limits the amount of excess PDDA available to interact with the AuNP surface, further leading to reduced aggregation. This assay does not serve our needs as a conclusive binding affinity was not established due to the incompatibility of monomeric $\alpha$-synuclein with AuNPs. 
Several binding studies were performed (EIS, CD, MST, AuNP colorimetric assay) to characterize the selectivity of ASYN2 towards monomeric $\alpha$-synuclein. $K_{d}$ values in both nanomolar range (EIS: $70 \pm 3 \mathrm{nM}$ ) and micromolar range (CD: $5.6 \mu \mathrm{M}$, MST: 5.7 $\mu \mathrm{M})$ were recorded while colorimetric assays proved incompatible for ASYN2 $\mathrm{K}_{\mathrm{d}}$ determination. The range of $\mathrm{K}_{\mathrm{d}}$ values obtained spans several orders of magnitude and is difficult to compare due to the aptamer being surface immobilized in EIS while free in solution within CD and MST studies. In cases of comparing different assays, it is important to revisit the initial selection parameters from which the aptamer candidates were generated and to identify the goal of this initial selection. ASYN aptamers were incubated with high concentrations of monomeric $\alpha$-synuclein to mimic overexpression that may be experienced within neuronal cells and to identify an aptamer that is capable of binding monomeric $\alpha$-synuclein at concentrations that will likely gravitate towards fibril formation. By selecting under these conditions, the probability for generating aptamer candidates exhibiting increased fibril inhibition potential is significantly greater at the cost of reduced target affinity. Dissociation constants determined from CD and MST studies are expected to resemble $\alpha$-synuclein concentrations experienced by the initial aptamer selection pool as ASYN remained free in solution to interact with its specific target domain.

EIS, on the other hand, would not be representative of the expected $\mathrm{K}_{d}$ for ASYN(15) as the surface immobilized aptamer is sterically restricted, limiting its conformational freedom for interacting with monomeric $\alpha$-synuclein. The selectivity of immobilized ASYN aptamers could be compared with similar literature aptasensing platforms to determine the efficacy and detection limitations of the fabricated aptasensor. The high sensitivity of EIS, paired with the potential for receptor clustering along the electrode 
interface, would contribute to the lower detection limits observed using this characterization method.

However, ASYN2 remains a potential therapeutic agent for fibril inhibition and the next step is to minimize ASYN2; determining if truncated forms retain the same binding and inhibition properties for monomeric $\alpha$-synuclein as the full-length sequence.

\subsection{ASYN2 minimer design}

Based on the success of ASYN2 in previous sections, ASYN2 was minimized in an effort to reduce synthesis and future therapy costs while determining if truncated sections improve binding and inhibition characteristics. The full length ASYN2 aptamer was truncated into various segments to exploit their unique secondary structures and compare binding affinity with respect to the full-length parent strand. The region, or regions, within ASYN2 primarily responsible for binding of monomeric $\alpha$-synuclein remain unknown, resulting in various segments being designed and tested to determine if they are an integral component of protein interaction (Figure 3.44).

Six minimers were designed, maintaining the secondary structure within the left side of the sequence (A2m1, bases 1-41), maintaining the secondary structure within the right side of the sequence (A2m2, bases 43-66), removal of the 3'-PBR (A2m3, bases 148), removal of the 5'-PBR (A2m4, bases 19-66), removal of both 5'- and 3'-PBRs (A2m5, bases 19-48), and maintaining a portion of the central randomized region predicted to contain a stable G-quadruplex secondary structure (A2m6, bases 34-47). From previous electrochemical studies, electrochemical impedance spectroscopy (EIS) suggested that availability of the 5'-PBR and 3'-PBRs within ASYN2 impact protein binding when 
immobilized on a gold electrode surface. The importance of conserving the 5'-PBR with the G-quadruplex domain, 3'-PBR with the G-quadruplex domain, and G-quadruplex domain in the absence of PBRs will be investigated using UV-Vis and CD to determine if a minimer possesses equivalent or higher affinity than ASYN2 for its monomeric target.

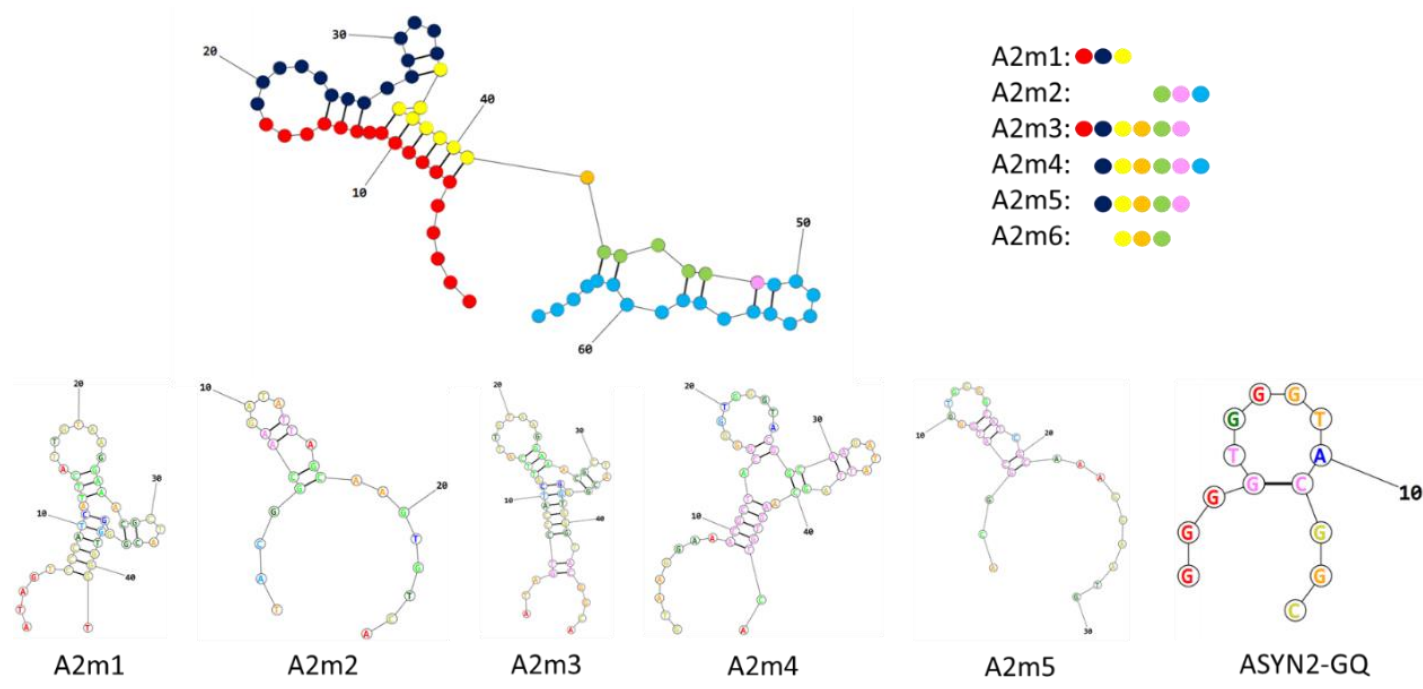

Figure 3. 44: Predicted secondary structure of ASYN2 according to RNAstructure software with labelled minimer sequences: 5'-stem loop structure (A2m1), 3'-stem loop structure (A2m2), no 3'-PBR (A2m3), no 5'-PBR (A2m4), no 5'- or 3'-PBRs (A2m5), centralized G-quadruplex binding motif (A2m6).

\subsubsection{A2m(1-6) melting temperature analysis}

Designed minimers were analyzed using UV-Vis spectroscopy to determine their respective melting temperatures $\left(T_{\mathrm{m}}\right)$ and whether they contain optimal G-quadruplex secondary structure for target binding. Over the range of 200-300 nm, UV absorption is a result in transitions of the pyrimidine and purine bases. ${ }^{227}$ Aptamers containing higher melting temperatures are more ideal as they exhibit increased stability over a broader temperature range for experimental conditions. Figure 3.45A-F illustrates the $T_{\mathrm{m}}$ plots for the six ASYN minimers in BFR-1 at $295 \mathrm{~nm}$ as they are subjected to a temperature gradient from $20^{\circ} \mathrm{C}$ to $90^{\circ} \mathrm{C}$. 
As predicted in Figure 3.44, A2 $\mathrm{m} 1$ and $\mathrm{A} 2 \mathrm{~m} 2$ are the respective left and right components of the full length ASYN2 sequence. Due to their sequence truncations the Gquadruplex motif found within full-length ASYN2 is not retained within these minimers, which is apparent by the lack of hypochromicity within the UV spectra. The $T_{\mathrm{m}}$ 's of A2m(3-6) all displayed hypochromicity as predicted, indicating that the G-quadruplex region has been conserved. Upon thermal denaturation, characteristic hypochromicity was observed in the range of $47^{\circ} \mathrm{C}-57^{\circ} \mathrm{C}(\mathrm{A} 2 \mathrm{~m} 3), 45^{\circ} \mathrm{C}-55^{\circ} \mathrm{C}(\mathrm{A} 2 \mathrm{~m} 4), 55^{\circ} \mathrm{C}-65^{\circ} \mathrm{C}(\mathrm{A} 2 \mathrm{~m} 5)$, and $65^{\circ} \mathrm{C}-75^{\circ} \mathrm{C}$ (A2m6) respectively, corresponding to G-quadruplex dissociation. ${ }^{228}$ Following the decrease in absorbance at $295 \mathrm{~nm}$, an increase in absorbance can be observed for minimers $\mathrm{A} 2 \mathrm{~m}(3-6)$ signifying the denaturation of ssDNA helical structure.

Experimental Table 2.1 displays predicted theoretical $T_{\mathrm{m}}$ values obtained from IDT where the minimers with the lowest $T_{\mathrm{m}}$ 's, $\mathrm{A} 2 \mathrm{~m} 2\left(56.1^{\circ} \mathrm{C}\right)$ and $\mathrm{A} 2 \mathrm{~m} 6\left(56.0^{\circ} \mathrm{C}\right)$, also have the lowest degree of secondary structure present. This suggests that the absence of the 5'stem-loop structure may decrease the stability of the minimer sequence and is observed in Figure 3.45F. A2 $\mathrm{m} 6$ is a minimer designed to encompass the G-quadruplex binding domain within the ASYN2 sequence, comprising 14 bases in length. From A2m6's $T_{\mathrm{m}}$, the initial denaturation cycle indicated the presence of a G-quadruplex, but upon sequential cooling and heating cycles, the secondary structure was not recovered. Lack of reproducibility indicates that $\mathrm{A} 2 \mathrm{~m} 6$ is not a stable sequence, potentially due to its shorter length of 14 bases when compared with other minimers. The non-reversible $T_{\mathrm{m}}$ of A2m6 can also be observed in Figure 3.45F, which is referred to as the hysteresis phenomenon. ${ }^{229}$ The hysteresis phenomenon is due to a slower structural transition within the minimer when compared to the changing temperature gradient. The minimers A2m(3-5) have the highest 
degree of secondary structure, corresponding to higher $T_{\mathrm{m}}$ 's according to IDT prediction software. This suggests that the presence or portion of the 5'-stem loop results in a secondary structure with greater stability. ${ }^{230}$
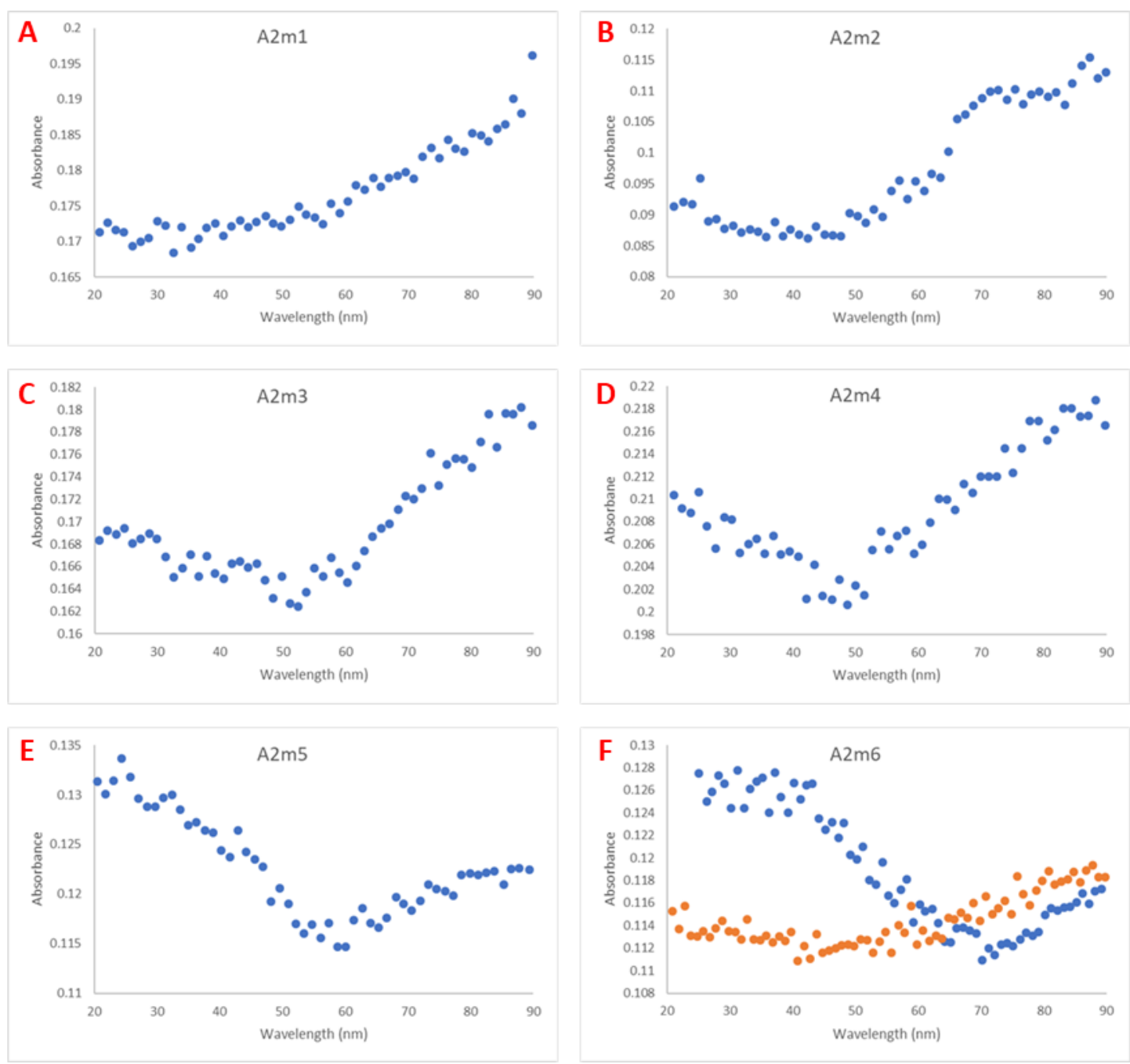

Figure 3. 45: Minimer melting temperature $\left(T_{m}\right)$ analysis at $295 \mathrm{~nm}$ over a temperature range of $20^{\circ} \mathrm{C}$ $-90^{\circ} \mathrm{C}$ : (A) A2m1, (B) A2m2, (C) A2m3, (D) A2m4, (E) A2m5, (F) A2m6. Hypochromicity is observed in $\mathrm{C}-\mathrm{F}$ indicated the denaturing of the centralized G-quadruplex motif. A2m6 displays two curves: increasing temperature ramp (blue curve) and decreasing temperature ramp (orange curve). The nonsuperimposable curves indicate hysteresis among the two curves and reflects the potential instability of A2m6. 


\subsection{CD spectroscopy of respective A2m(1-6) minimers}

The CD spectra of ASYN2 minimers, A2m(1-6), were also analyzed to determine if the truncated sequences contain a G-rich sequence capable of forming the G-quadruplex binding domain observed in the parent strand (Figure 3.46).

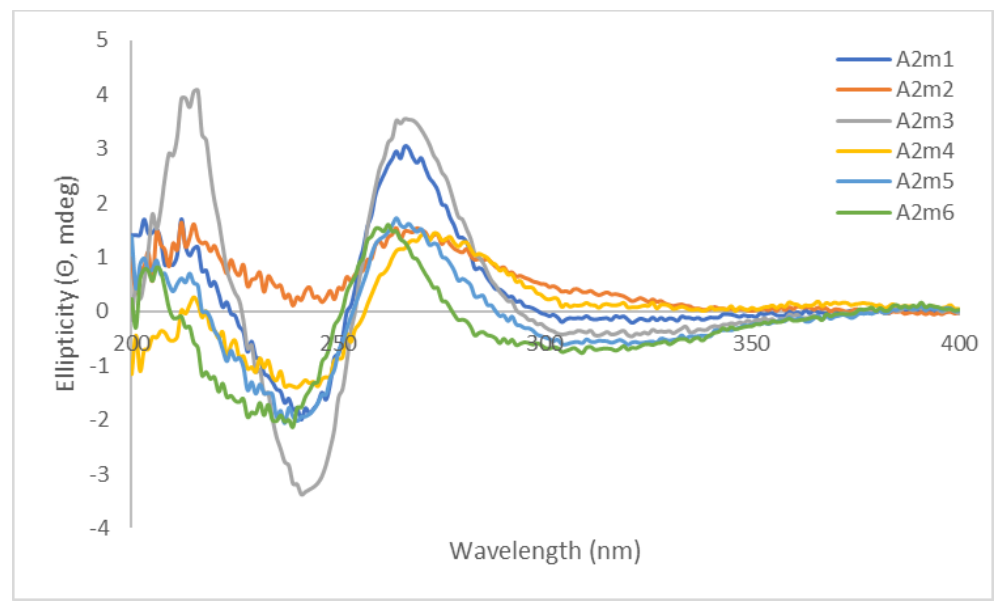

Figure 3. 46: CD spectra of A2m(1-6) sequences at a concentration of $18.75 \mu \mathrm{M}$ in BFR-1.

$\mathrm{A} 2 \mathrm{~m} 1$ (bases 1-41) and A2m2 (bases 43-66) represent the two secondary structures within ASYN2. As these minimers sever the randomized region, both do not contain the guanine residues necessary for G-quadruplex formation. This determination is suggested by both the absence of a hypochromic shift in $T_{m}$ and the absence of a positive $216 \mathrm{~nm}$ peak in CD studies. A2m(3-6) contain a centralized G-quadruplex according to $T_{m}$ studies and can be distinguished by the presence of both $216 \mathrm{~nm}$ and $260 \mathrm{~nm}$ positive maxima. However, $\mathrm{CD}$ features within the individual sequences can isolate the prospective binding minimers from those unable to form a correct binding conformation. A2m4 indicated a Gquadruplex stability of $40-50^{\circ} \mathrm{C}$ from $T_{m}$ analysis, considerably lower than the other minimers. From the CD spectra of A2m4, a red-shift occurs at the $216 \mathrm{~nm}, 240 \mathrm{~nm}$, and 
$260 \mathrm{~nm}$ peaks relevant to a G-quadruplex conformation, suggesting G-quadruplex formation is not as stable in this minimer when compared with other minimers.

The minimer A2m6, designed specifically to encompass the G-quadruplex region within ASYN2, had a hypochromic peak between $70-80^{\circ} \mathrm{C}$ and was initially predicted to be the most stable minimer. A2m6's inability to reform the G-quadruplex structure during $T_{m}$ studies can be observed through its CD spectra as its relevant peaks are significantly blue shifted, further suggesting a lack of quadruplex structure. The reasoning behind the lack of A2m6's G-quadruplex was initially hypothesized to be due to its short sequence length of 13 bases, but $\mathrm{A} 2 \mathrm{~m} 5$ contains $\mathrm{A} 2 \mathrm{~m} 6$ along with the remainder of the randomized sequence encompassing 30 bases. The sequence length of A2m5 was believed to help stabilize the G-quadruplex through additional bases on either side of the binding motif, but its CD spectra indicates the relative lack of a $216 \mathrm{~nm}$ peak.

The final minimer, $\mathrm{A} 2 \mathrm{~m} 3$, absent of the $3^{\prime}-\mathrm{PBR}$, indicated a $50-60^{\circ} \mathrm{C}$ hypochromic shift in $T_{m}$ studies and the presence of a G-quadruplex through its $216 \mathrm{~nm}$ and $260 \mathrm{~nm}$ positive maxima. The $\mathrm{CD}$ maxima and minima for $\mathrm{A} 2 \mathrm{~m} 3$ are representative of those in ASYN2, containing a more intense maximum at $216 \mathrm{~nm}$ and suggesting a potential ASYN2 minimer candidate for target binding. One interesting aspect regarding the minimer $\mathrm{CD}$ spectra is that the two minimers containing the 5'-PBR, A2m1 and $\mathrm{A} 2 \mathrm{~m} 3$, contain the highest peak intensities at $220 \mathrm{~nm}, 240 \mathrm{~nm}$, and $260 \mathrm{~nm}$ compared with the other minimers. This suggests that the presence of the 5'-PBR within the minimer sequence contributes with folding and stabilization of the G-quadruplex structure when immersed in binding buffer. 


\subsection{In vitro $\alpha$-synuclein fibril inhibition through the use of ASYN aptamers}

Previous characterization studies on ASYN(1-5) and A2m(1-6) established binding affinity data for monomeric $\alpha$-synuclein using a variety of methods. Combining these supplementary data with the goal of inhibiting $\alpha$-synuclein fibril formation will provide further insight into the inhibition mechanisms of the various aptamer and minimer sequences as a potential therapeutic agent. Monomeric $\alpha$-synuclein protein in the presence of a preformed $\alpha$-synuclein fibril (PFF) was obtained and used for the following aptamer inhibition study. Literature has suggested the presence of a preformed fibril or misfolded monomeric protein can act as a template that mediates the oligomerization and fibril formation of native monomeric $\alpha$-synuclein. ${ }^{34-37}$ For this assay, $\alpha$-synuclein containing a PFF seed was incubated in the presence and absence of ASYN aptamers and minimers over a period of seven days at $37^{\circ} \mathrm{C}$ and constant agitation. Following the seven-day incubation period, TEM imaging was used to visualize the fibril formation that had occurred in the presence and absence of aptamers. Fibril inhibition observed with ASYN aptamers was then compared with several literature aptamers selected for $\alpha$-synuclein that were also found to inhibit fibril formation among monomeric $\alpha$-synuclein. ${ }^{105,142,143}$

Control studies were performed (Figure 3.47A-C) examining the TEM stain (1\% uranyl acetate), monomeric $\alpha$-synuclein, and a control aptamer Transferrin Receptor Aptamer (TRA) following the seven-day incubation period. As expected, uranyl acetate alone (UA) (Figure 3.47A) did not indicate any aggregation and formed a dark, uniform film along the carbon-copper grid. UA is commonly used for both positive and negative staining in electron microscopy as it is extremely electron dense with an atomic weight of 238 and is capable of interacting with proteins, lipids, and oligonucleotide structures. ${ }^{231,232}$ 
When dissolved in an aqueous solution UA can exist in many ionic complex states depending on the concentration and $\mathrm{pH}$ of solution, allowing the stain to effectively interact with cationic, anionic, and neutral structures. ${ }^{232}$ At $1 \%$ UA, the concentration is relatively low, and the $\mathrm{pH}$ of the solution will predominantly occur between 4.2 and 4.9. In this state cationic UA ions are readily available, frequently engaging with both the negatively charged DNA phosphate backbone along with the highly acidic C-terminal of $\alpha$ synuclein. ${ }^{232,233}$ The disadvantages observed with UA upon exposure to BFR-1 on the TEM grid, having a physiological $\mathrm{pH}$, induced $\mathrm{UA}$ to precipitate as uranium tetrahydroxide $\left(\mathrm{U}(\mathrm{OH})_{4}\right)$. Lack of an effective washing step following the addition of stain to the TEM grid resulted in residual UA salt in the TEM images, hindering the ability to see fibril formation at times and providing an unclean background. ${ }^{231-233}$ Future steps would be performed to mitigate these effects.
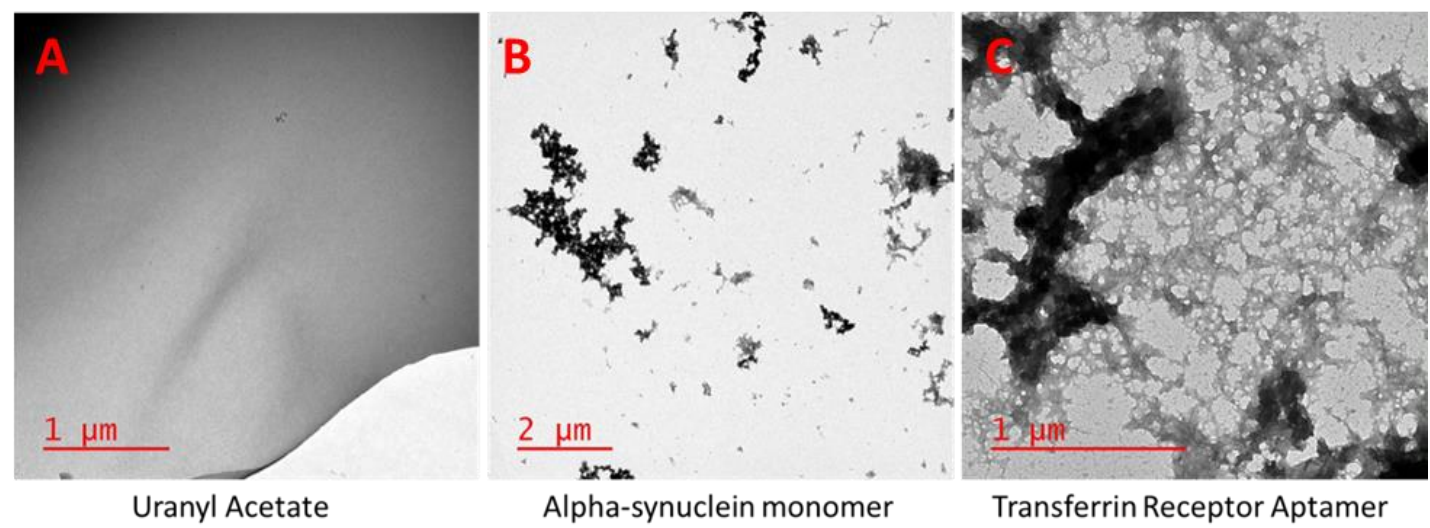

Transferrin Receptor Aptamer

Figure 3. 47: TEM images illustrating the uranyl acetate (UA) stain used to visualize the $\alpha$-synuclein fibril species (A), monomeric $\alpha$-synuclein following a seven-day incubation (B), random transferrin receptor aptamer (TRA) following a seven-day incubation in the presence of a PFF $\alpha$-synuclein seed protein. The seven-day incubation period was kept at $37^{\circ} \mathrm{C}$ and constant agitation. Scale bar, $1 \mu \mathrm{m}$.

It was also hypothesized that control monomeric $\alpha$-synuclein in the absence of a PFF seed would not undergo fibril formation, and that the TRA control would not inhibit 
fibril formation as it does not retain the necessary binding sequence or secondary structure as $\operatorname{ASYN}(1-5)$ aptamers. Figure 3.47B illustrates that monomeric $\alpha$-synuclein in the absence of PFF seeds did not undergo fibril formation after the seven-day incubation period. One study found that $\alpha$-synuclein incubated at $37^{\circ} \mathrm{C}$ without shaking led to the formation of an insoluble oligomeric species, which could explain the minor aggregation within the corresponding TEM image. ${ }^{37}$ TRA (Figure 3.47C) resulted in significant fibril formation as indicated by the presence of a dense, web-like structure encompassing majority of the available grid. Lack of specific binding regions within the TRA aptamer would have had minimal effect in preventing the eventual fibrillization of $\alpha$-synuclein.

In the absence of aptamer, $\alpha$-synuclein fibril formation can be observed in Figure 3.48 alongside ASYN(1-5) and the reference aptamer M5-15 following a seven-day incubation period. ${ }^{142}$ Mature, fully formed fibril structures are illustrated in (A) as long, continuous web-like interlocking structures spanning greater than $3 \mu \mathrm{m}$. Compared to fibril formation in the absence of DNA aptamers, the presence of ASYN(1-5) and M5-15 (B-G) in aptamer:protein molar ratios of 1:1 indicate binding inhibition to varying degrees where ASYN2 was determined to be the most inhibitive. All aptamers were able to effectively inhibit fibril formation at aptamer:protein molar ratios of 10:1, but only ASYN2 could successively prevent fibrillization to a recognizable degree at 1:1 molar ratios. ASYN1, 3, 4, and 5 (D, E, F, G) appeared to have minimal effects on inhibiting the formation of fibril structure at a:1 aptamer:protein molar ratios as the dense, web-like fibril structures can be easily discerned from that of ASYN2 (B).

It appears ASYN2 prevented the fibrillization of monomeric $\alpha$-synuclein but resulted in the formation of smaller premature fibril complexes or oligomeric species. The 
exact mechanism of aggregate formation is unclear and additional tests would need to be performed to determine whether the cytotoxicity of these smaller structures is lesser or greater than the presence of a fully developed $\alpha$-synuclein fibril in neuronal cells. Literature has suggested that the presence of small fibril structures less than $50 \mathrm{~nm}$ are most detrimental to the misfolding and transmission of $\alpha$-synuclein to adjacent cells by acting as a seed that initiates prion-like conversion of native $\alpha$-synuclein into an aggregate species. ${ }^{37}$ It was suggested in a second study ${ }^{36}$ that $\alpha$-synuclein PFF were endocytosed into a neuronal cell in vitro without the need for additional assistance where it induced misfolding of endogenous $\alpha$-synuclein into an aggregate species. Following a seven-day period, the aggregates accumulated in length and resembled LNs, another distinct feature of $\alpha$-synucleinopathies. ${ }^{36}$

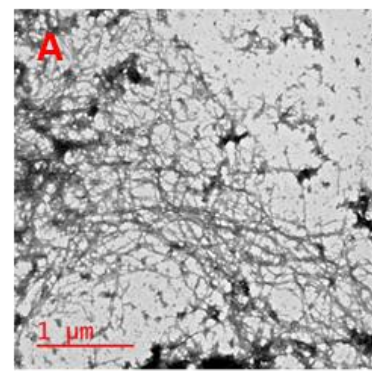

PFF

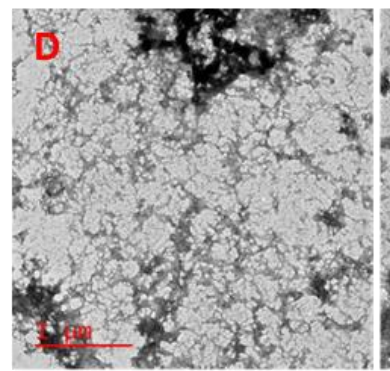

ASYN1

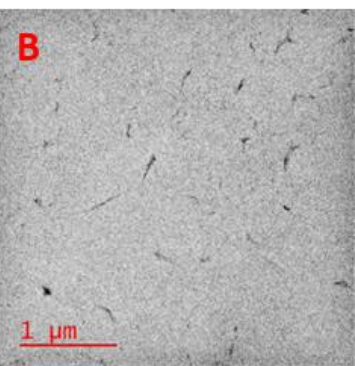

ASYN2

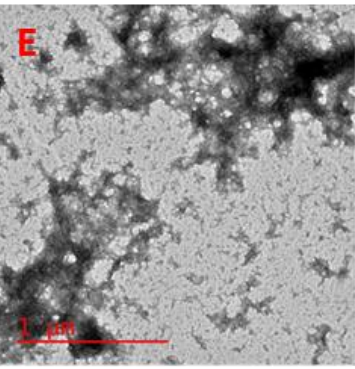

ASYN3

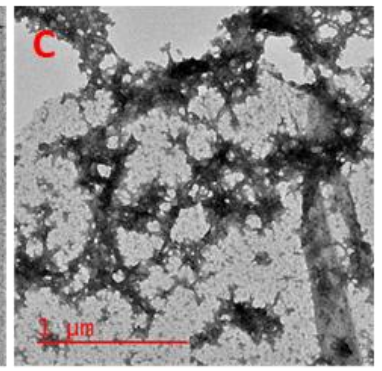

M5-15

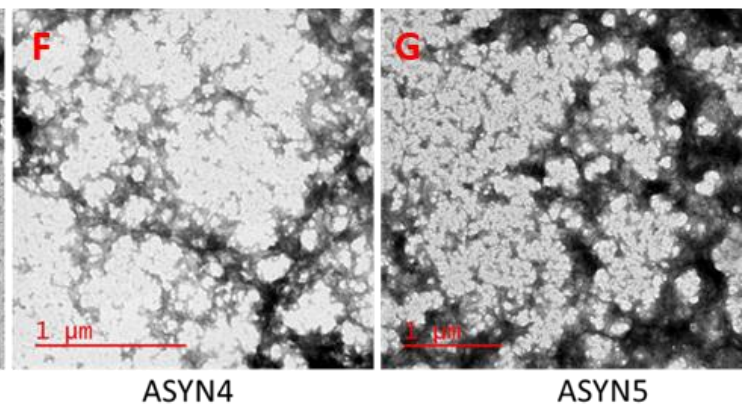

Figure 3. 48: TEM images comparing the inhibitive effects of ASYN(1-5) and M5-15 on monomeric $\alpha$ synuclein fibril formation. The respective aptamers were incubated for seven days in the presence of an $\alpha$-synuclein PFF at $37^{\circ} \mathrm{C}$ and with constant agitation. (A) $\alpha$-synuclein fibrils (PFF) in the absence of aptamer, (B) ASYN2, (C) M5-15, (D) ASYN1, (E) ASYN3, (F) ASYN4, (G) ASYN5. All images were obtained in aptamer:protein molar ratios of 10:1. Scale bar, $1 \mu \mathrm{m}$. 
ASYN2 was compared with aptamers from literature, F5R1 and F5R2, ${ }^{143}$ and two M5-15 minimers, T-SO508 and T-SO530, ${ }^{105}$ in 10:1 molar ratios of aptamer:protein to determine how ASYN2 compares with other aptamers from the literature (Figure 3.49AE).

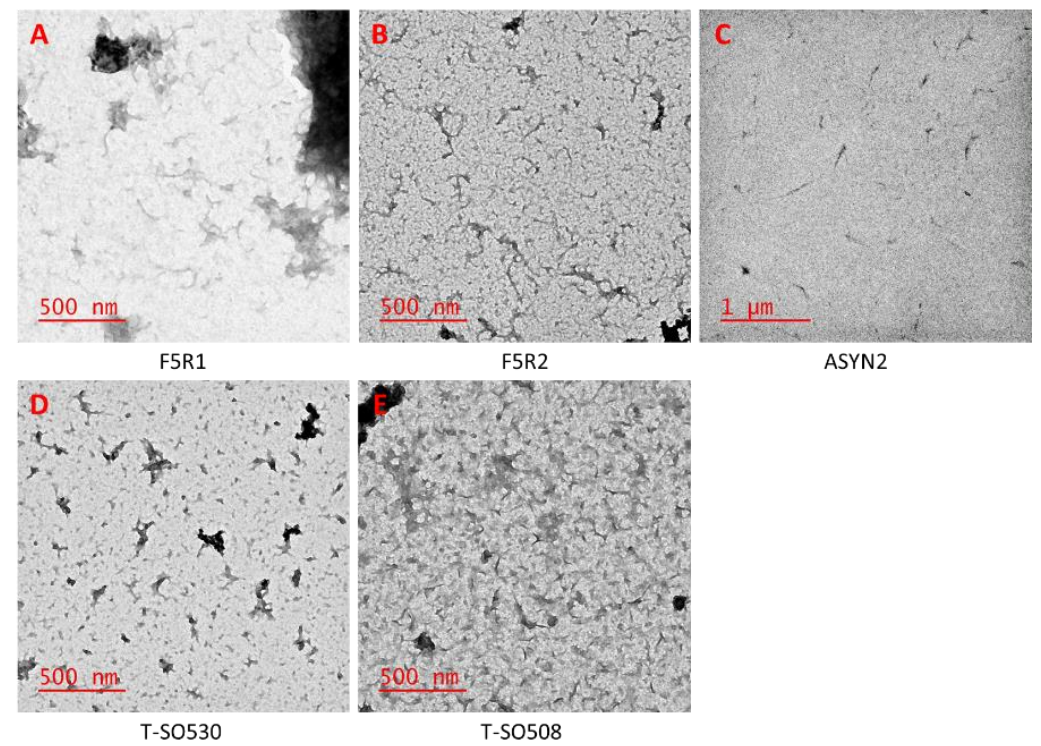

Figure 3. 49: TEM images comparing the aggregation inhibition of ASYN2 with respect to literature aptamers selected for monomeric and oligomeric $\alpha$-synuclein: (A) F5R1, (B) F5R2, (C) ASYN2, (D) TSO530, (E) T-SO508. All images were obtained in aptamer:protein molar ratios of 10:1 following a seven-day incubation period at $37^{\circ} \mathrm{C}$ and constant agitation. Scale bar, $1 \mu \mathrm{m}$.

The four literature aptamers were selected for the oligomeric form of $\alpha$-synuclein and has respective $\mathrm{K}_{\mathrm{d}}$ values of 2.40 and $3.07 \mathrm{nM},{ }^{143} 68$ and $63 \mathrm{nM} .{ }^{105}$ Aptamer:protein molar ratios of 10:1 were used in this instance as the F5R1 study ${ }^{143}$ found that successful inhibition was observed in excess of 10:1 ratios, but 5:1 molar ratios was significantly less efficient. The aggregate structures share a close resemblance to one another following the incubation period, with T-SO508 (E) leading to a higher density of oligomeric-like $\alpha$ synuclein aggregates that are not observed in (A-C). 
To determine the inhibition limitations of ASYN2 on $\alpha$-synuclein fibril formation, various molar ratios were analyzed from 10:1 to 0.1:1 aptamer:protein (Figure 3.50A-D).
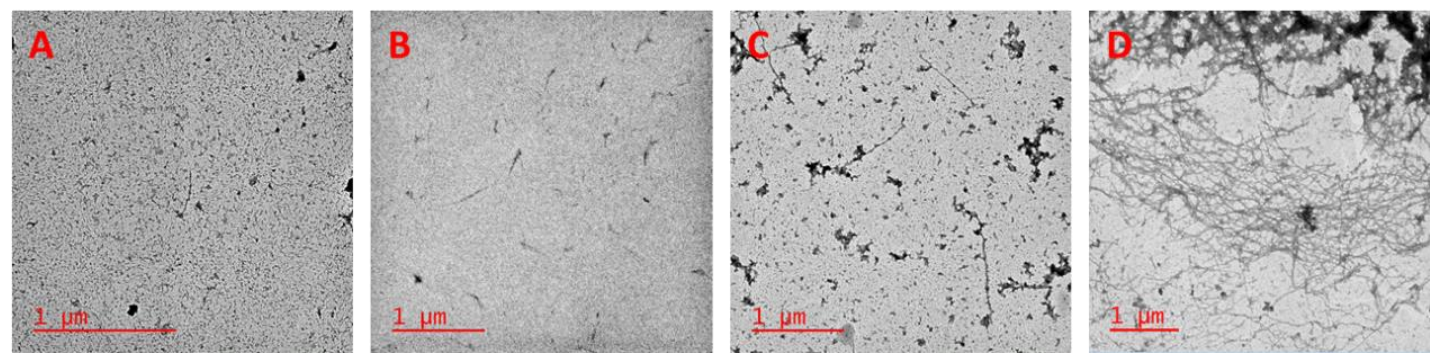

Figure 3. 50: TEM images of the inhibition potential of ASYN2 in molar ratios of ASYN2: $\alpha$-synuclein PFF of: (A) 10:1, (B) 1:1, (C) 0.5:1, (D) 0.1:1. All images were obtained following a seven-day incubation period at $37^{\circ} \mathrm{C}$ and constant agitation. Scale bar, $1 \mu \mathrm{m}$.

At 10:1 aptamer:protein, minimal fibril formation occurred due to an excess of ASYN2 protein to interact with the monomeric protein, hindering its ability to adopt a misfolded conformation. As the molar concentration is reduced to $0.5: 1$, the relative size of the fibril structures has significantly lengthened to over twice the initial length at $1: 1$ molar concentration. ASYN2 is still having a protective effect on $\alpha$-synuclein, but due to the reduced concentration available it is likely localized aptamer was completely consumed. Further reducing the molar ratio to $0.1: 1$ aptamer;protein (D) produced a mature fibril structure almost identical to $\alpha$-synuclein PFF in the absence of aptamer.

The exact binding mechanism between ASYN2 and $\alpha$-synuclein remains unknown, but the binding domain integral for this aptamer-protein interaction can be postulated. Antibody studies examining the formation of $\alpha$-synuclein fibrils found that antibodies capable of recognizing the $\mathrm{N}$-terminal or $\mathrm{C}$-terminal domains of monomeric $\alpha$-synuclein could inhibit subsequent aggregation. ${ }^{234-236}$ Epitope mapping into the sequence components responsible for $\alpha$-synuclein fibril formation revealed varying stain intensities of antibodies along the aggregated protein structure. ${ }^{237,238}$ Amino acids 11-26 of the N- 
terminal region and $108-131$ of the C-terminal region were most intensely labeled, suggesting that these regions remain exposed upon fibril formation while the other regions, presumably involved in the aggregate structure, are embedded or degraded and are not accessible for staining. ${ }^{238}$ Literature has also identified a hydrophobic region within the NAC domain of $\alpha$-synuclein spanning amino acid residues 71-82 (VTGVTAVAQKTV) integral for fibril formation. ${ }^{239}$ The presence of this NAC fraction alone is capable of seeding $\alpha$-synuclein aggregation, suggesting the possibility of a binding domain for ASYN2 at either of these three regions within $\alpha$-synuclein. ${ }^{35}$

As described earlier, performed EIS studies between immobilized ASYN2 and $\alpha$-, $\beta$-, $\gamma$ - synuclein and fibril $\alpha$-synuclein (Figure 3.19) indicated high affinity for $\alpha$-synuclein $(640 \Omega)$, while both $\beta$-synuclein $(60 \Omega)$ and the fibril $(202 \Omega)$ had minor interactions while $\gamma$ - synuclein (15 $\Omega$ ) displayed non-specific to no interaction with ASYN2. Literature has suggested $\beta$ - and $\gamma$ - synuclein cannot assemble into fibril structures, even following a fiveweek incubation period. ${ }^{239,240}$ Reasoning to support this is that both $\beta$ - and $\gamma$ - synuclein do not possess the necessary hydrophobic region amino acids 71-82 as $\alpha$-synuclein (Figure 3.22). This could imply the NAC region comprising amino acids $71-82$ as a potential region for specific binding. However, because this region is directly involved in fibril formation and minor affinity was observed between ASYN2 and fibril aggregates, it is not as likely that this region can be attributed to the specificity of ASYN2. Comparing the N-terminal and C-terminal regions between $\alpha-\beta$-, and $\gamma$ - synuclein reveals a lack of structural similarity, leading to additional regions of interest for aptamer-target interactions. Supporting literature suggests long-range interactions exist between the N-terminal and Cterminal regions that enable the correct conformation for the progression of fibril 
formation. ${ }^{143,241,242}$ By minimizing the conditions necessary for long-range interactions to exist, the amount of visible aggregation significantly decreased. ${ }^{243,244}$ Specific interactions between ASYN2 and $\alpha$-synuclein could exist between both $\mathrm{N}$ - and C-terminals, explaining the minor affinity expressed previously.

A2m(1-6) minimers $\alpha$-synuclein inhibition potential was also demonstrated and compared to full length ASYN2 (Figure 3.51A-F).

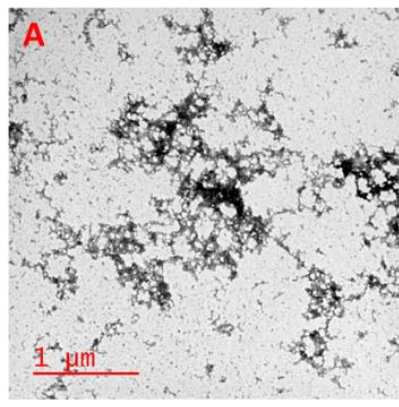

$\mathrm{A} 2 \mathrm{~m} 1$

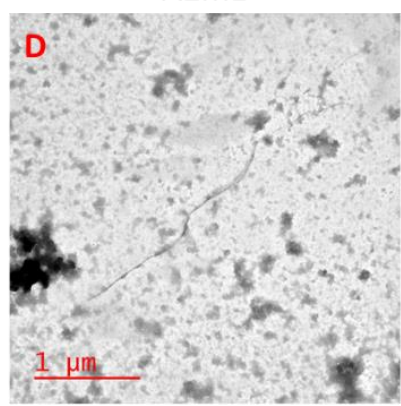

$\mathrm{A} 2 \mathrm{~m} 4$

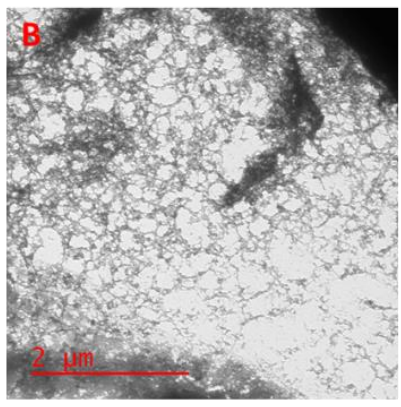

$\mathrm{A} 2 \mathrm{~m} 2$

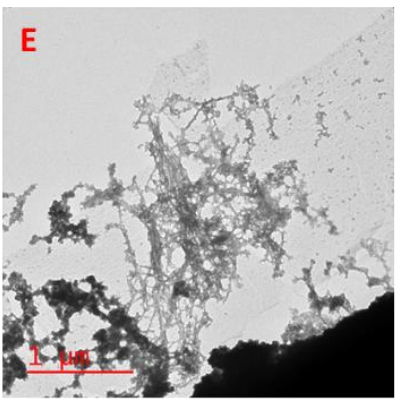

$\mathrm{A} 2 \mathrm{m5}$

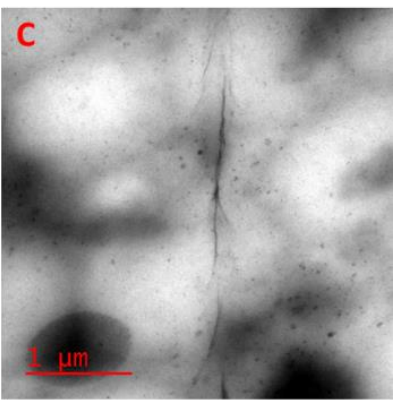

$\mathrm{A} 2 \mathrm{~m} 3$

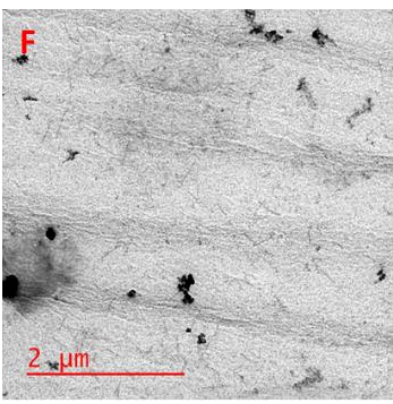

$\mathrm{A} 2 \mathrm{~m} 6$

Figure 3. 51: TEM images comparing the inhibitive effects of $A 2 m(1-6)$ on monomeric $\alpha$-synuclein fibril formation. The respective aptamers were incubated for seven days in the presence of an $\alpha-$ synuclein PFF at $37^{\circ} \mathrm{C}$ and with constant agitation. (A) A2m1, (B) A2m2, (C) A2m3, (D) A2m4, (E) A2m5, (F) A2m6. All images were obtained in aptamer:protein molar ratios of 1:1. Scale bar, $1 \mu \mathrm{m}$.

As expected, A2m1 (A) and A2m2 (B) appeared to have negligible effect on fibril inhibition as did A2m5 (E) despite $T_{m}$ studies indicating the presence of a favorable Gquadruplex binding conformation. A2m3 (C), A2m4 (D), and A2m6 (F) exhibited ability to minimize the association of $\alpha$-synuclein into larger aggregates by the lack of dense fibril networks and presence of individual fibril strands. Contrasting ASYN2, the individual 
fibril strands obtained in the $\mathrm{A} 2 \mathrm{~m} 3$ and $\mathrm{A} 2 \mathrm{~m} 4$ studies surpass $3 \mu \mathrm{m}$ but suggest binding between minimer and protein. $\mathrm{A} 2 \mathrm{~m} 6$, on the other hand, produces oligomeric or protofibril species similarly produced when $\alpha$-synuclein was subjected to ASYN2. Despite A2m6's ability to inhibit fibril progression, $T_{m}$ studies reveal the unstable nature of this minimer. Further evaluation of $\mathrm{A} 2 \mathrm{~m} 3$ could prove beneficial in developing an aptamer containing equivalent binding and inhibiting properties as the full length ASYN2 sequence while minimizing steric hindrance and increasing rotational freedom in solution. 


\section{Chapter 4: Contributions to Knowledge and Future Direction}

This research highlighted various characterization methods describing the binding affinity, conformation, and target interactions of generated monomeric $\alpha$-synuclein aptamers, ASYN(1-5), alongside the development and optimization of electrochemical and optical aptasensors. The application of EIS as a biosensing platform provided reproducible, inexpensive, and sensitive analysis of the molecular interactions occurring at a gold electrode interface between ASYN(1-5) and monomeric $\alpha$-synuclein. Effective optimization of aptasensor components and environmental parameters provided the foundation for obtaining $\mathrm{K}_{d}$ values of ASYN(1-5) and ASYN2R in the nanomolar range. ASYN2 was determined to have the highest binding affinity, closely comparing to literature data on selected aptamers against monomeric and oligomeric species of $\alpha$ synuclein. Several papers suggest immobilizing AuNPs on the surface of the AuE to increase the amount of available surface area for thiolated DNA to tether. ${ }^{156,174,245,246}$ The presence of AuNPs significantly enhances the sensitivity of the aptasensor by promoting electron transfer within the reaction and producing more observable changes in $\mathrm{R}_{\mathrm{ct}}$.

MST data indicated ASYN2 retained similar binding affinities for both monomeric and A53T variants of $\alpha$-synuclein, displaying inherent advantages and disadvantages associated with this aptamer. The ability for ASYN2 to bind with both monomeric and A53T species suggests a lack of specificity in its target of preference, but in a therapeutic sense proves advantageous as the end goal of ASYN2 development is to inhibit monomeric species of $\alpha$-synuclein from progressing into larger aggregate structures.

Colorimetric assays involving AuNPs have accumulated popularity in literature due to the optical properties associated with their specific plasmon resonance. A visual colour 
change from red to blue accompanying distinct absorbance spectra distinguishing colloidal AuNPs, $525 \mathrm{~nm}$, and aggregated AuNPs, $680 \mathrm{~nm}$, yielded attractive properties for optical biosensing. Substitution of $\mathrm{NaCl}$ for PDDA to induce AuNP aggregation enabled more vibrant colour among AuNP samples and finer control of the biosensor components. It was determined, however, that AuNP-based colorimetric assays were not a suitable detection method for monomeric $\alpha$-synuclein detection as the protein prevented PDDA-induced aggregation. Alterations to the $\mathrm{pH}$ and ionic components of the buffer environment could potentially make colorimetric sensing compatible with the detection of monomeric $\alpha$ synuclein.

Design of minimer sequences exploiting the predicted binding domains of ASYN2 yielded six minimers labelled A2m(1-6). $T_{m}$ analysis at $295 \mathrm{~nm}$ determined whether the minimer sequences were capable of adopting a G-quadruplex motif, predicted to be an integral component for specific binding of monomeric $\alpha$-synuclein. A stable and conserved G-quadruplex was present in $\mathrm{A} 2 \mathrm{~m}(3-5)$, placing further interest on these three minimer sequences as a potential replacement for ASYN2 as a therapeutic agent.

CD spectra of ASYN(1-5) and A2m(1-6) provided conformational analysis of the respective sequences in the presence and absence of monomeric $\alpha$-synuclein. A parallel Gquadruplex motif was predominantly observed within ASYN2, ASYN4, and A2m3 under physiological conditions. A calibration curve was performed on ASYN2 in the presence of increasing monomeric $\alpha$-synuclein concentrations where a $\mathrm{K}_{\mathrm{d}}$ of similar magnitude to MST results was obtained. Further efforts into the exploration of specific A2m3 interactions with monomeric $\alpha$-synuclein could determine how the minimers specific binding compares to the parent ASYN2 sequence. 
In vitro inhibition studies involving monomeric $\alpha$-synuclein in the presence of a PFF seed protein were performed on generated full-length ASYN(1-5), respective A2m(16) minimers, and literature aptamers selected against monomeric and oligomeric $\alpha$ synuclein. Following a seven-day incubation period TEM imaging revealed ASYN(1-5) sequences could effectively inhibit fibril progression at 10:1 molar ratios (aptamer:protein) while only ASYN2 could prevent aggregation as low as 1:1, with longer fibril structures emerging at 0.5:1 and mature fibril formation occurring at 0.1:1 molar ratios. A2m(1-6) minimers were all performed at 1:1 molar ratios where it was determined that $\mathrm{A} 2 \mathrm{~m} 3$, $\mathrm{A} 2 \mathrm{~m} 4$, and $\mathrm{A} 2 \mathrm{~m} 6$ provided optimal inhibition results for further study. The ability for A2m3 to exhibit G-quadruplex stability while inhibiting aggregate species formation require further analysis into the interaction mechanism and binding potential of the minimer sequence with respect to the full-length strand.

Current and future work revolves around encapsulating ASYN2 into a liposomal drug delivery vessel capable of migrating through the blood brain barrier and analyzing its therapeutic potential in vivo. Substitution of ASYN2 for the truncated minimer A2m3 could prove beneficial if $\mathrm{A} 2 \mathrm{~m} 3$ is found to have similar binding affinity as its parent strand. The smaller size would also lead to a higher abundance of encapsulated sequences, providing increased inhibition potential upon monomeric $\alpha$-synuclein proximity within neuronal cells. Further in vitro examination into the specific binding mechanism of ASYN2 with monomeric $\alpha$-synuclein could be explored by comparing its affinity for individual regions within the full-length protein sequence. ASYN2 affinity for truncated sections of monomeric $\alpha$-synuclein spanning the $\mathrm{N}$-terminal, NAC, and C-terminal domains respectively would provide mechanistic insights into potential modes of fibril inhibition. 
Application of isothermal titration calorimetry, a gold standard in aptamer binding affinity analysis, would provide reliable data surrounding the thermodynamics and specific molecular interactions of aptamer-target complexation. With these supplementary studies, understanding into the specific chemistry involved between ASYN2 and monomeric $\alpha$ synuclein could further enhance its potential as a therapeutic strategy for PD. 


\section{Appendices}

\section{Appendix A Development of therapeutic strategies for ASYN2 drug delivery}

\section{A.1 Introduction}

The blood-brain barrier (BBB) is composed of endothelial cells lining cerebral capillaries that are essential for maintaining the internal microenvironment and strict regulation of foreign species permeability. ${ }^{247,248}$ The high surface area within the brain provides numerous available sites for exchange into the brain that can disrupt functionality, making this physical barrier vital for human survival. Alternatively, the impenetrable nature of the BBB poses a significant challenge when designing therapeutic approaches for directly treating brain diseases as the BBB will inhibit drugs access into the brain. There are two methods of entering the brain through the BBB: the transcellular pathway directly through the plasma membrane of endothelial cells and paracellular pathway through channel of adjacent endothelial cells. ${ }^{247,248}$ The transcellular pathway utilizes various modes of diffusion, active transport and transcytosis to effectively uptake substances into the brain. ${ }^{247,248}$ An abundance of carrier- and receptor-mediated transport systems exist throughout endothelial cells, resulting in the interest and exploitation of the transferrin receptor (TR) for access across the BBB. ${ }^{249}$

TRs primary function is to mediate endocytosis of iron into endothelial cells through coupling with transferrin. ${ }^{250}$ TRs are expressed in cells throughout the body, but the rate of oxidative metabolism within neuronal cells requires an abundance of iron, resulting in the overexpression of TRs along endothelial cells. ${ }^{251}$ The availability of TRs from the blood stream has made them a therapeutic access point for drug delivery from 
which several DNA and RNA aptamers have been characterized and applied for novel delivery methods.

Chen et al. ${ }^{252}$ generated the initial DNA and RNA mouse TR aptamers (TRA) for the purpose of examining the endocytosis of lysosomal enzymes conjugated with TRA across the BBB. DNA aptamers exhibited greater stability throughout in vitro studies over RNA aptamer and is illustrated in Figure 5.1A as GS24. It was determined GS24, termed herein as TRA, was non-competitive with transferrin-iron complexes, retained a binding affinity of $0.25 \mu \mathrm{M}$, and postulated that it could provide a successful avenue for transcytosis delivery methods. ${ }^{252}$

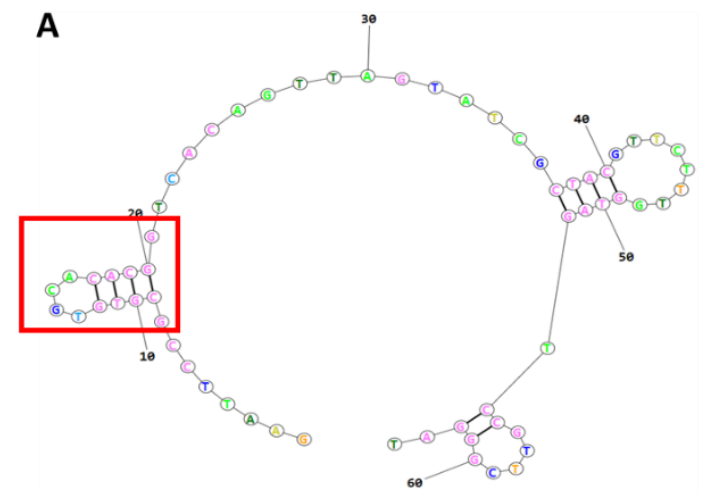

B

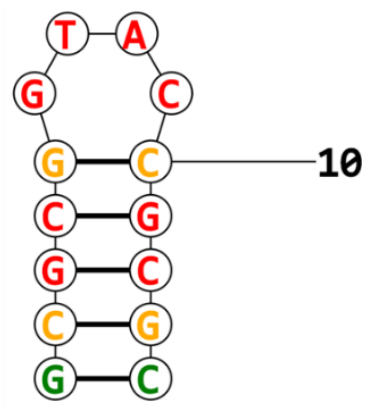

C

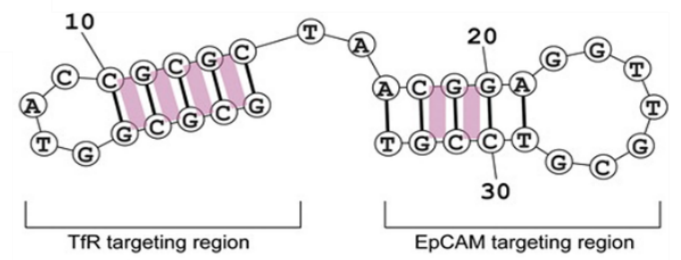

Figure 5. 1: Literature DNA aptamers exhibiting specific binding for transferrin receptors along endothelial cells. A) Full-length transferrin receptor aptamer (TRA); B) truncated TfR minimer exploiting the 5'-stem loop within the full-length sequence; C) therapeutic bifunctional aptamer utilizing a chimera between $\mathrm{TfR}$ and specific drug delivery sequence.

A truncated form of TRA retained a secondary structure of two stem-loops that were believed responsible for its interactions with TR. Conformational analysis of TRA 
was performed by one group ${ }^{253}$ where they discovered its ability to interact with TR is predominantly influenced by the presence of a stem-loop at the 5'-terminal. By creating mutant TRA sequences through the substitution of bases in predicted binding regions, they determined a conserved motif of 14 bases believed to be responsible for TR binding. ${ }^{253,254}$ This TRA minimer, termed herein as TfR, was characterized and enhanced by a third group $^{254-256}$ where minor base changes within the minimer sequence significantly improved the binding affinity to $0.11 \mu \mathrm{M}$ Figure 5.1B. It was also found that $\mathrm{TfR}$ could successfully transcytose the BBB and was further applied as a drug delivery strategy for brain cancer (Figure 5.1C). A bifunctional chimera aptamer capable of targeting both transferrin and epithelial cell adhesion molecules (EpCAM) was developed that acted as a drug carrier for the anthracycline chemotherapeutic doxorubicin (DOX). ${ }^{255,256}$ The bifunctional aptamer had six potential sites for DOX intercalation, and was found to successfully cross the BBB, delivering its cargo to EpCAM-positive cancer cells. ${ }^{256}$ Alongside this novel method of drug delivery, traditional methods such as liposomes have been well-established as a drug delivery strategy.

Initially developed in the $1960 \mathrm{~s},{ }^{257}$ liposomes have been extensively used across the field of nanomedicine for their attractive properties as drug delivery vessels. ${ }^{258-260}$ Composed of various phospholipid structures, liposomes are amphiphilic in nature and form an enclosed sphere with two distinct regions when immersed in aqueous solutions. The polar phosphate head groups of phospholipids orient themselves in a way to minimize unfavourable thermodynamic interactions with the solvent, resulting in a hydrophilic core and lipophilic bilayer that are stabilized through the hydrophobic effect and corresponding van der Waals interactions among its acyl chains. Several classes of liposomes have been 
distinguished such as small unilamellar vesicles (SUVs), large unilamellar vesicles (LUVs), and multilamellar vesicles (MUV) ${ }^{258}$ In this section, the Bangham method of thin film hydration is described to synthesize conventional SUV liposomes between 20-100 nm in diameter. ${ }^{257}$ Phospholipids were initially dissolved in an organic solvent, chloroform, where the solvent is gradually evaporated to generate the thin lipid film. Aqueous rehydration of the thin lipid film with gentle agitation results in the formation of large unilamellar vesicles (GUVs) containing the therapeutic agent, ASYN2. Water bath sonication and extrusion respectively reduce the liposome size to form homogenous SUVs containing the therapeutic aptamer. ${ }^{257,258}$

Liposomes have been thoroughly characterized throughout literature for their biocompatibility, nontoxic nature, and their ability to be chemically modified for specific targets. ${ }^{258-260}$ Depending on the properties of the therapeutic drug, it can be encapsulated within both the lipid bilayer and the aqueous core making liposomes very attractive for delivering large quantities of drug to a localized region. Several approved liposome-drug combinations are currently in use: Myocet is used as a DOX delivery vessel for metastatic breast cancer, ${ }^{261}$ and DepoDur for the delivery of morphine sulfate in pain management. ${ }^{262}$

\section{Section Objectives}

This chapter describes the synthesis and characterization of two potential drug delivery methods of ASYN2. The first approach was encapsulating ASYN2-Cy5 aptamer within SUVs containing surface modifications for the specific interaction with TR. Injection into mice and characterization of aptamer concentration within neuronal tissues would be used to assess the efficacy of this delivery method for in vivo applications. The 
second approach was to develop a bifunctional chimera aptamer containing both ASYN2 and an aptamer for TR. Several aptamers were characterized to assess their stability and affinity for the desired target. 


\section{A.2 Experimental}

\section{Materials and reagents}

Phospholipids 1-palmitoyl-2-oleoyl-glycero-3-phosphocholine (16:0-18:1 PC, POPC), dimethyl dioctadecyl ammonium bromide salt (18:0 DDAB), 1,2-distearoyl-snglycero-3-phosphoethanolamine-N-[methoxy(polyethylene glycol)-2000] (ammonium salt) (18:0 DSPE-PEG 2000 PE), and 1,2-distearoyl-sn-glycero-3-phosphoethanolamine$\mathrm{N}$-[maleimide(polyethylene glycol)-2000] (ammonium salt) (DSPE-PEG 2000 Maleimide) for liposome synthesis were obtained from Avanti Polar Lipids Inc (Alabama, USA). Ferric chloride hexahydrate and ammonium thiocyanate were obtained from Sigma Aldrich (Germany). Slide_-Lyzer dialysis cassettes with a 20K molecular weight cut-off were purchased from ThermoFisher Scientific (Canada). A liposome extruder set consisting of a mini-extruder, two $250 \mu \mathrm{L}$ gas-tight glass syringes, $10 \mathrm{~mm}$ filter supports, and $0.1 \mu \mathrm{m}$ PC membranes was purchased from Millipore Sigma (Germany). Analytical grade, anhydrous ethanol and chloroform were obtained from commercial sources. Bovine RNase-free DNase I was purchased from ThermoFisher Scientific. Micro bio-spin buffer exchange columns were obtained from Bio-rad (USA). Various buffers were prepared throughout the liposome synthesis. DNA was dissolved in $50 \mathrm{mM}$ Tris-HCl, pH 7.0 (BFR5), dialysis was performed in $50 \mathrm{mM}$ HEPES, pH 7.0 (BFR-6), DNA encapsulation occurred in 67\% ethanol in $50 \mathrm{mM}$ Tris-HCl, pH 7.0 (BFR-7), disulfide cleavage occurred in $50 \mathrm{mM}$ Tris-HCl, $100 \mathrm{mM}$ DTT, pH 8.4 (BFR-8), buffer exchange buffer containing 50 mM HEPES, 7 mM EDTA, pH 7.0 (BFR-9). All solutions were prepared in deionized water $\left(\mathrm{DiH}_{2} \mathrm{O}, 18.0 \mathrm{M} \Omega \cdot \mathrm{cm}\right.$ resistivity) from a Millipore MilliQ system. 


\section{DNA oligonucleotide synthesis}

Aptamer sequences are listed in Table 5.1. Aptamers involved in liposome synthesis, ASYN2 and transferrin receptor aptamer (TRA), were purchased from Integrated DNA Technologies (IDT, Coralville, IA). ASYN2 was labelled with a Cy5 fluorophore and TRA was modified with a C6 S-S disulfide at their 5'-terminals before being prepared to their desired concentrations in BFR-5 and BFR-8 respectively. Bifunctional chimera aptamers were prepared using standard phosphoramidite chemistry on a BioAutomation Corporation MerMade 6 DNA Synthesizer (USA) at a $1 \mu$ mol scale with $1000 \AA$ controlled pore glass (CPG) columns. Standard manufacturer synthesis, purification, and quantification conditions are described previously in Experimental 2.2.

Table 5. 1: List of modified aptamer sequences involved in liposome synthesis where colored segments (Cy 5, orange) and (C6-S-S, green) denote the 5' modifications.

\begin{tabular}{|c|l|}
\hline Aptamer & \multicolumn{1}{c|}{ Sequence $\left(\mathbf{5}^{\prime} \rightarrow \mathbf{3}^{\prime}\right)$} \\
\hline ASYN2-Cy5 & $\begin{array}{l}\text { 5'- Cy 5 - ATA GTC CCA TCA TTC ATT GTA AGG AAA CGC TAC } \\
\text { GGG GTG GGT ACG GCA AGA TAT TAG CAA GTG TCA }\end{array}$ \\
\hline TRA-C6-S-S & $\begin{array}{l}\text { 5' - C6 S-S - GAA TTC CGC GTG TGC ACA CGG TCA CAG TTA } \\
\text { GTA TCG CTA CGT TCT TTG GTA GTC CGT TCG GGA T }\end{array}$ \\
\hline
\end{tabular}

\section{Liposome synthesis}

Glassware was washed in warm, soapy water (Sunlight brand dish soap) for $15 \mathrm{~min}$, rinsed 25 times with tap water, 10 times with distilled water, and five times with $\mathrm{ddH}_{2} \mathrm{O}$ before drying overnight. POPC (100 mg, $8 \mathrm{~mL})$, DDAB (1.0 mg, $1 \mathrm{~mL})$, and DSPE-PEG 2000 maleimide $(1.0 \mathrm{mg}, 1 \mathrm{~mL})$ were dissolved in anhydrous chloroform to form lipid stock solutions. POPC (1.17 mL), DDAB (126.3 $\mu \mathrm{L})$, DSPE-PEG 2000 maleimide $(88.25$ $\mu \mathrm{L})$, and DSPE-PEG $2000(1.7 \mathrm{mg})$ were added to a $10 \mathrm{~mL}$ round bottom flask that was septum capped and wrapped in parafilm. Residual chloroform was evaporated through 
slow agitation and continuous argon flow for $2 \mathrm{~h}$ before the lipid film was rehydrated in BFR-5 $(200 \mu \mathrm{L})$ for $30 \mathrm{~min}$ and sonicated for $10 \mathrm{~min}$ in a water bath sonicator. ASYN2Cy5 (38 nmol, $200 \mu \mathrm{L})$ was encapsulated within liposomes through the gradual addition of BFR-7 $(600 \mu \mathrm{L})$. The liposome/DNA solution was subjected to 10 freeze-thaw cycles consisting of an ethanol/dry ice bath for $5 \mathrm{~min}$ and water bath at $40^{\circ} \mathrm{C}$ for $2 \mathrm{~min}$. Liposomes were extruded 51 times through $0.1 \mu \mathrm{m}$ PC membrane of a handheld extruder and placed overnight in a $20 \mathrm{~K}$ dialysis cassette immersed in BFR-6. The liposome solution was removed from the dialysis cassette and reacted with $10 \mu \mathrm{L}$ DNase I in reaction buffer for 15 min before $5 \mu \mathrm{L}$ stop buffer was introduced. The liposome solution was placed in a second 20K dialysis cassette immersed in BFR-6 for $6 \mathrm{~h}$ with fresh buffer exchange occurring every $30 \mathrm{~min}$. TRA (120 nmol, $75 \mu \mathrm{L})$ suspended in BFR-8 was incubated for 30 min with constant agitation before buffer exchanging to BFR-9 in micro bio-spin columns. Four cycles at $1 \mathrm{G}$ and four min in length collected the cleaved TRA which were directly added to $3 \mathrm{~K}$ centrifuge columns for $20 \mathrm{~min}$ at $10 \mathrm{G}$. TRA was collected by inverting the microcentrifuge columns for four min at $1 \mathrm{G}$ before being added to the liposome sample. A final overnight dialysis was performed in $20 \mathrm{~K}$ cassettes immersed in BFR- 6 .

\section{Quantification of synthesized liposomes}

Following an overnight dialysis, ASYN2-Cy5 encapsulated liposomes containing exterior conjugated TRA were quantified via UV-Vis spectroscopy. A 20x dilution was examined at $590 \mathrm{~nm}$ to quantify the $\mathrm{Cy} 5$ concentration, while a 200x dilution was examined at $260 \mathrm{~nm}$ to quantify the concentration of TRA immobilized on the liposome surface. 
A Stewart assay was performed according to J.C.M. Stewart ${ }^{263}$ and is described briefly. A stock liposome solution $(11.94 \mathrm{mg} / \mathrm{mL})$ comprised of POPC (1.17 mL), DDAB (126.3 $\mu \mathrm{L})$, DSPE-PEG 2000 maleimide (88.25 $\mu \mathrm{L})$, and DSPE-PEG 2000 (1.7 mg) was prepared. A standard liposome solution $(0.1 \mathrm{mg} / \mathrm{mL})$ was prepared in $3 \mathrm{~mL}$ chloroform from the liposome stock solution. Ferrithiocyanate reagent was prepared by dissolving ferric chloride hexahydrate $(0.5406 \mathrm{~g})$ and ammonium thiocyanate $(0.6080 \mathrm{~g})$ in $\mathrm{ddH}_{2} \mathrm{O}$ $(20 \mathrm{~mL})$. A standard curve was developed where equivalent volumes of ferrothiocyanate $(250 \mu \mathrm{L})$ were added to each sample containing diluted liposome solution. Samples were vortexed for $20 \mathrm{~s}$ and centrifuged at $1000 \mathrm{RPM}$ for $10 \mathrm{~min}$ prior to UV-Vis analysis. Absorbances were performed in triplicate and quantified at $485 \mathrm{~nm}$ by analyzing the chloroform layer of the standard curve solutions. Once a standard curve was developed, synthesized liposomes were performed in triplicate at $1 \mathrm{x}$ and 10x dilutions. 


\section{A.3 Results and Discussion}

\section{Statement of contribution}

Liposome synthesis protocol was initially developed by Dr. Erin McConnell and Dr. Maria DeRosa. ASYN2-Cy5 loaded liposomes were synthesized by Spencer Boisjoli, Vernon Hunt, and Anna Koudrina. In vivo injection and experimentation were performed by Katelyn Ventura and Dr. Matthew Holahan. Chimera bifunctional aptamers were designed by Spencer Boisjoli and Dr. Maria DeRosa.

\section{Liposome composition and synthesis}

The application of liposomes as a delivery vessel disguises the chemical characteristics of the aptamer therapeutic and prevents the requirement of designing a specialized small molecule, $<400 \mathrm{Da}$, for payload delivery beyond the $\mathrm{BBB}$. The liposomal lipid foundation consisted of POPC, DDAB, DSPE-PEG(2000), and DSPE-PEG(2000) maleimide to ensure biocompatibility and successful transcytosis across the plasma membrane of the BBB Figure 5.2.

POPC lipids are commonly used in liposome synthesis as they are zwitterionic at physiological $\mathrm{pH}$, allowing them to self-assemble into lamellar structures independently of the solvent properties. ${ }^{258}$ POPC contains both saturated and unsaturated acyl chains that resemble the composition of in vivo cell plasma membranes. The similarity in phospholipid composition may provide better integration of the synthesized liposomes with the target cells upon drug delivery. 


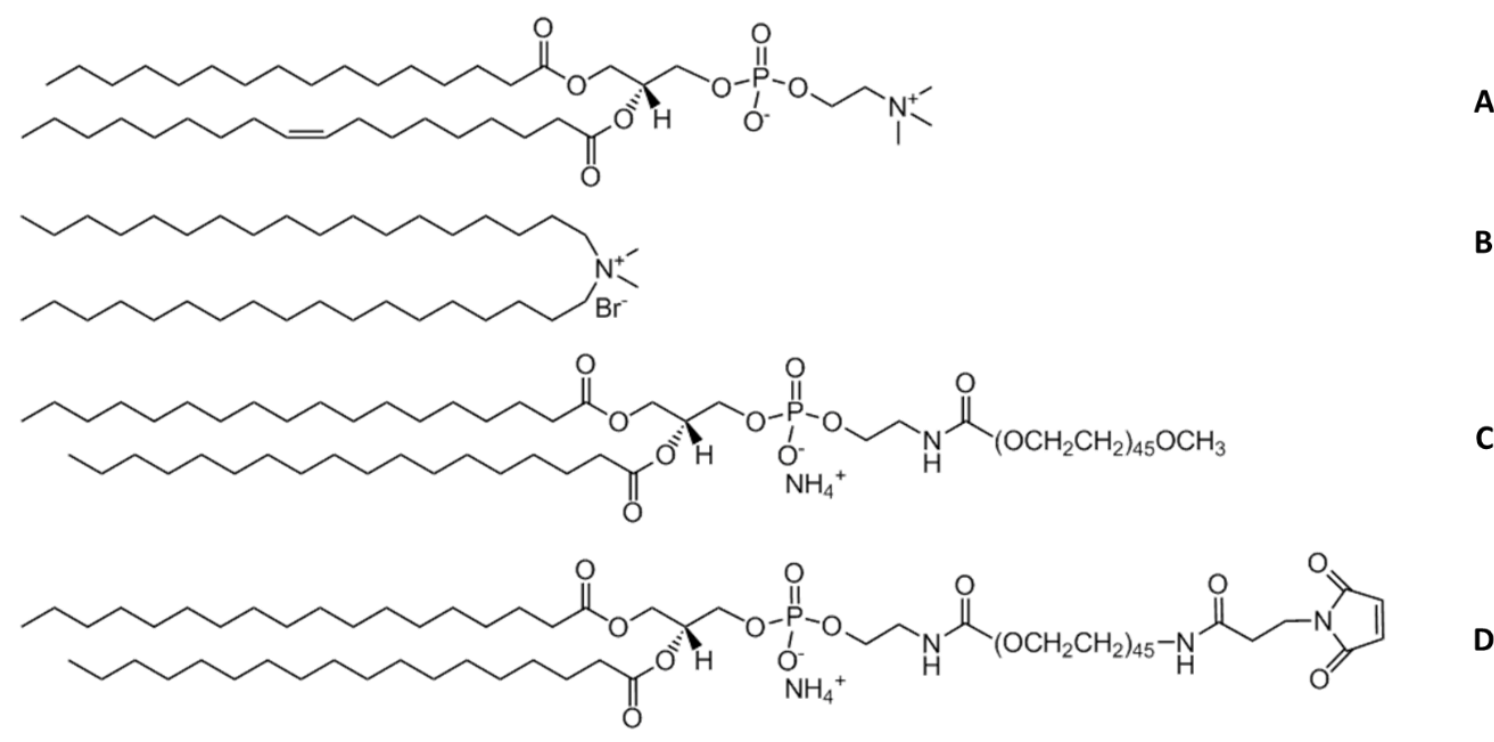

Figure 5. 2: Lipids involved in liposome synthesis: A) POPC; B) DDAB; C) DSPE-PEG(2000); D) DSPE-PEG(2000) maleimide

The electrochemical composition of a liposome surface can be modified via cationic, anionic, or neutral lipids, and the presence of DDAB within the liposome bilayer incorporates cationic properties to the surface. Literature has suggested cationic lipids prevent liposome aggregation due to electrostatic repulsion and can better interact with negatively charged cell membranes and DNA, increasing the efficiency of drug delivery. ${ }^{264,265}$ Disadvantages have also been associated with electrochemically charged liposomes regarding longevity, clearance rate, and toxicity. ${ }^{266}$ Intravenous administration of the liposome delivery system is hindered by the mononuclear phagocyte system (MPS), predominantly situated in the liver and spleen, that rapidly removes foreign agents from the bloodstream. ${ }^{260,266}$ The rapid opsonization of liposome vessels destabilizes the phospholipid bilayer and results in the premature release of the aptamer payload.

Liposome surface modifications regarding the presence of a polymer coating have been adopted in synthesis methodologies to maximize both stability and circulation half- 
life of liposome vessels in the bloodstream. ${ }^{258,260,266}$ The introduction of a hydrophilic polyethylene glycol (PEG) polymer in the form of DSPE-PEG(2000) is the main contributor in the steric stabilization of liposomes. Termed "stealth liposomes", the neutral and flexible properties of surface immobilized PEG creates a steric barrier that masks the liposome from rapid MPS recognition. ${ }^{259,260,266}$ The final lipid used for fabricating the liposome vessels was DSPE-PEG(2000) modified with a terminal maleimide ligand. In the presence of a terminal sulfhydryl within TRA, a stable thioether bond is formed at physiological $\mathrm{pH} .{ }^{249}$ This reaction covalently tethers TRA to the liposome, allowing the liposome to engage with endothelial TR and penetrate the BBB.

During liposome synthesis phospholipid phase transitions could occur within the thin lipid film that induce physical changes in bilayer composition. Depending on the phase transition temperature of the respective lipids, they can exist in a disordered fluid state ( $T$ $\left.>T_{p t t}\right)$ or an ordered gel state $\left(T<T_{p t t}\right) .258,267$ As the synthesized liposomes are primarily composed of POPC and modified DSPE, these two lipids will contribute most to the phase transition temperature. From literature, the $T_{p t t}$ of POPC and DSPE are suggested to be $3^{\circ} \mathrm{C}^{268}$ and $74^{\circ} \mathrm{C}^{269}$ respectively. These $T_{p t t}$ reflect the chemical composition of the respective lipid structures. DSPE contains two saturated acyl chains while POPC contains both saturated and unsaturated acyl chains. The degree of saturation within DSPE allows for closer packing to occur during bilayer formation, corresponding to a higher $T_{p t t}$. The presence of an unsaturated acyl chain in POPC generates a kinked structure that minimizes packing density and increases mobility of the bilayer. Considering the influence POPC and DSPE exert on overall liposome $T_{p t t}$, the average $T_{p t t}$ is postulated to be similar to physiological temperatures. Literature has suggested that liposomes in the fluid state are 
more susceptible to drug encapsulation. ${ }^{258}$ As aptamer encapsulation occurred within a $40^{\circ} \mathrm{C}$ water bath, it is possible that the liposomes existed in a fluid state above their $T_{p t t}$ that is more susceptible to aptamer encapsulation. Upon administration in vivo, physiological temperatures of $37^{\circ} \mathrm{C}$ could induce a transition in liposome composition from a fluid state to a gel state, preventing the leakage or premature release of encapsulated ASYN2.

\section{Liposome quantification}

$\mathrm{UV}-\mathrm{V}$ is spectroscopy was performed to quantify the efficiency of therapeutic agent encapsulation, the presence of surface immobilized TRA, and overall liposome synthesis. A UV-Vis spectra depicting concentrations of encapsulated and surface immobilized DNA is illustrated in Figure 5.3.

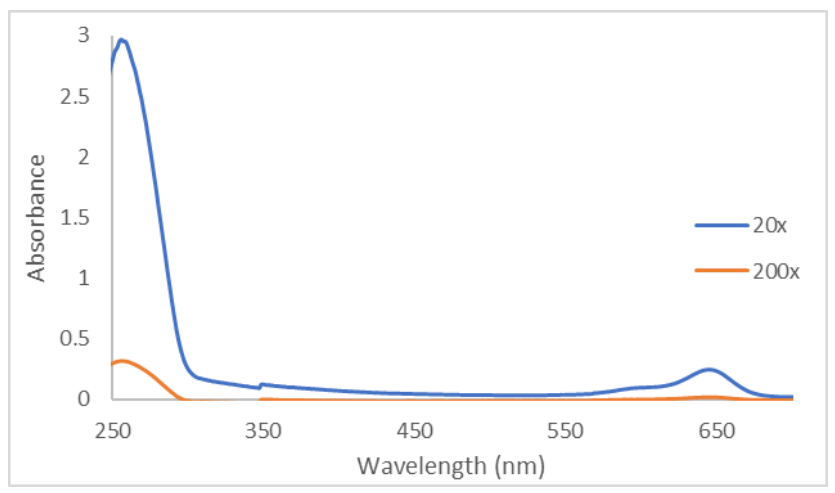

Figure 5. 3: Quantification of encapsulated ASYN2 (20x dilution, blue curve) and tethered exterior TRA (200x dilution, orange curve) following liposome synthesis.

A 20x dilution was performed to examine the Cy5 peak, based on its characteristic absorbance at $646 \mathrm{~nm}$, where it was determined a total concentration of $18.4 \mu \mathrm{M}$ ASYN2Cy5 was encapsulated within the liposomes. This concentration equates to $64.5 \mathrm{nmol}$ of encapsulated ASYN2, or approximately 53.7\% loading efficiency. One strategy described in literature for increasing encapsulation efficiency was the reverse phase evaporation 
method. ${ }^{270}$ In brief, reverse phase evaporation generates inverted micelles through the sonication of an aqueous-organic mixture where the therapeutic agent is dissolved in the aqueous phase and the lipids are dissolved in the organic phase. Excess phospholipids aid in the formation of the lipid bilayer to complete the formation of the liposome vessel, resulting in a large aqueous core and high encapsulation efficiencies. ${ }^{270}$

With respect to determining the total concentration of immobilized TRA on the liposome surface, a 200x dilution was performed to quantify the $260 \mathrm{~nm}$ DNA peak. A total concentration of $95.2 \mu \mathrm{M}$ DNA was detected, approximately $76.7 \mu \mathrm{M}$ (268 nmol) of which represented immobilized TRA. The covalent interaction between TRA and maleimide modified liposomes was approximately $74.6 \%$, indicating that surface saturation was likely reached.

The efficiency of the liposome synthesis was to be quantified through a Stewart assay to determine the overall composition of lipids within the synthesized sample. A 0.1 $\mathrm{mg} / \mathrm{mL}$ lipid standard was prepared by dissolving POPC, DDAB, DSPE-PEG(2000), and DSPE-PEG(2000) maleimide in chloroform. The inorganic ferrothiocyanate compound was introduced as an aqueous phase to the organic lipid mixture. Ferrothiocyanate is insoluble in chloroform but readily complexes in the presence of phospholipids, partitioning within the organic layer. ${ }^{263}$ Unfortunately, current quarantine protocol has prevented the synthesized liposome batch to be quantified, so Figure 5.4 depicts a Stewart assay of a prior synthesis to illustrate what is expected. A 10x liposome dilution (orange) correlates to a final concentration of $25.1 \pm 0.61 \mathrm{mg} / \mathrm{mL}$, indicating an approximate yield of $70 \%$. 

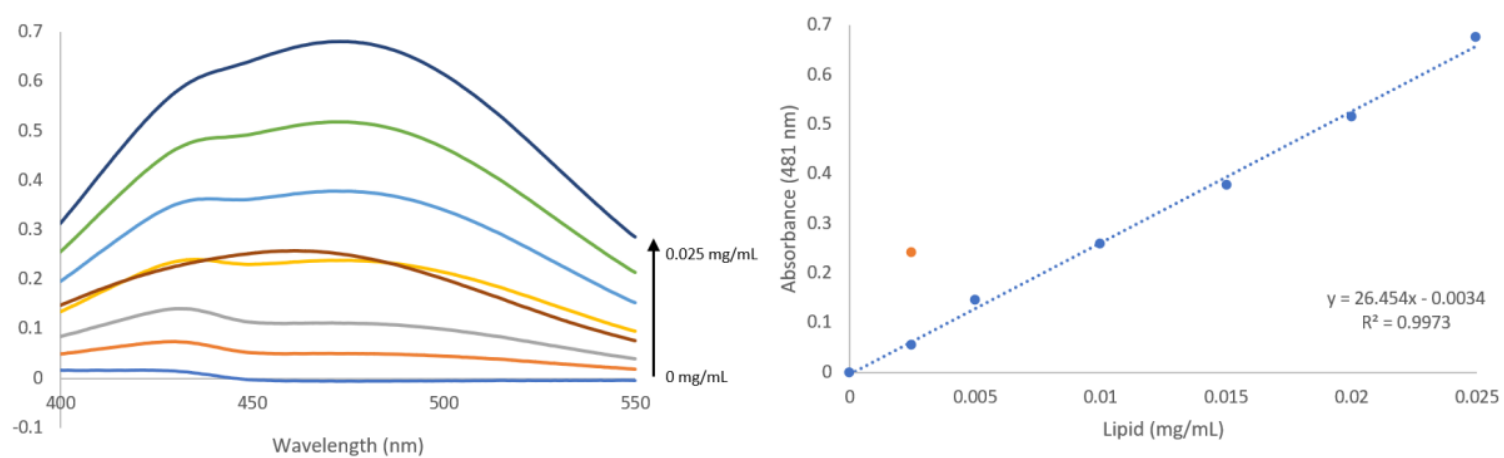

Figure 5. 4: Stewart assay results used to determine the final lipid composition of the previously synthesized liposomes. A calibration curve was produced over a lipid concentration of $0-0.025 \mathrm{mg} / \mathrm{mL}$ at a constant concentration of ferrothiocyanate. The orange dot in the right figure corresponds to the lipid concentration of the synthesized liposomes, determined to have a final concentration of $25.1 \pm 0.61$ $\mathrm{mg} / \mathrm{mL}$ ( $70 \%$ yield).

\section{Chimera bifunctional aptamer design}

The primary goal driving bifunctional aptamer development containing a specialized TR aptamer, TRA and TfR, and the therapeutic agent, ASYN2, was to further reduce production time and cost requirements (Figure 5.5). Two aptamers were identified from literature that expressed affinity for TR; the initial full-length TR sequence referred to as TRA and the truncated sequence referred to as TfR that encompasses the stem-loop believed responsible for interacting with TR. When designing a chimera aptamer, it was vital that both aptamers within the chimeric sequence adopt their initial binding conformation upon exposure to their respective targets without inhibiting the other. Previous experimental data suggest a binding domain for monomeric $\alpha$-synuclein within ASYN2 located at the 5' stem loop. Exploiting this binding motif, four chimera aptamers were designed for ASYN2 and A2m3, labelled ASYN2-TRA, ASYN2-TfR, A2m3-TRA, and A2m3-TfR respectively. Chimera aptamers containing TRA displayed characteristics that negate their bifunctional application, specifically due to the length of TRA, resulting in an increase in potential base pairing interactions. Within both ASYN2- and A2m3-TRA 
chimeras, the predicted G-quadruplex motif is hindered as a result of intramolecular base pairing with TRA. Loss of G-quadruplex secondary structure will minimize affinity for monomeric $\alpha$-synuclein while simultaneously disrupting the binding domain for transferrin. Due to the above properties coupled with the aptamer's length and preparation restrictions, ASYN2-TRA and A2m3-TRA chimera aptamers do not seem plausible moving forward.

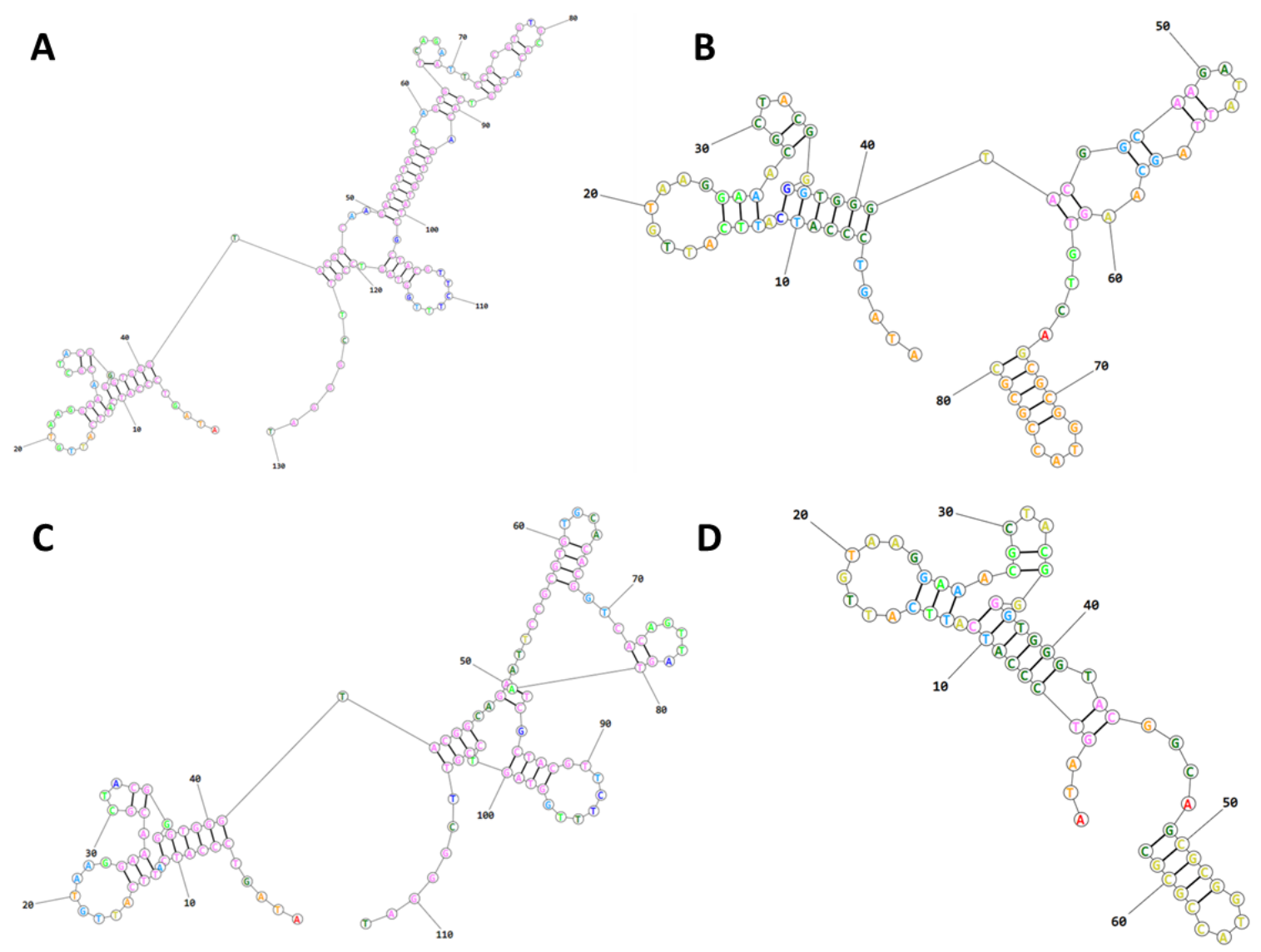

Figure 5. 5: Theoretical chimera aptamers: A) ASYN2-TRA; B) ASYN2-TfR; C) A2m3-TRA; D) A2m3-TfR.

Contrary to the above discussion, substitution of TRA with the truncated sequence TfR lead to ideal predicted secondary structures when combined within the bifunctional sequence. Both G-quadruplex domains of ASYN2 and A2m3 were readily available, the 
potential 5'-terminal binding domain for monomeric $\alpha$-synuclein was not hindered through TfR coupling, and the TfR stem-loop was retained for potential binding with TR. One issue that may arise from the A2m3-TfR chimera is the available G-quadruplex conformations within its sequence. According to QGRS mapping software, four potential regions exhibiting a G-score of 18 were located within this chimera aptamer that are not present within ASYN2-TfR. This results from the absence of a 3'-PBR within A2m3, reducing overall steric hindrance within the aptamer and allowing the TfR domain to be involved in the G-quadruplex motif due to its G-rich nature. Characterization into which G-quadruplex motif is favoured is essential as it could result in loss of the TR stem-loop. The stability of the G-quadruplex would also need to be assessed as change in temperature and environmental conditions could lead to a more thermodynamically favourable conformation. 


\section{A.4 Conclusions and Future Direction}

ASYN2-encapsulated SUV liposomes were synthesized, and bifunctional chimera aptamers designed for potential in vivo therapeutic treatment strategy for PD. Due to Covid-19, the progression of this project was significantly hampered. However, further studies into liposomal physiological stability, ASYN2 release and localization at the designated site, and clearance rates would further support the efficacy of this form of treatment. With respect to the bifunctional aptamers, ASYN2-TfR and A2m3-TfR displayed attractive secondary properties that require further characterization studies to assess if this will be a reliable avenue of approach for PD treatment. Some hypothesized studies include $T_{m}$ analysis on both chimera sequences with and without monomeric $\alpha-$ synuclein and TR to determine their stability and how their conformation, specifically ASYN2 G-quadruplex and TfR stem-loop, are affected; CD studies can identify the presence of G-quadruplex secondary structure and how both G-quadruplex and stem-loop change in the presence of their respective targets; PFF inhibition assays would determine whether the presence of TfR is detrimental to ASYN2 and A2m3's ability to hinder the progression of monomeric $\alpha$-synuclein fibrils; fluorescent modification of the chimera 5'terminal would enable fluorescent tracking of the aptamer and determine if the presence of ASYN2 or A2m3 impact the ability of TfR to interact with TR and on the sequences ability to undergo transcytosis across an endothelial cell. 


\section{Appendix B Mass spectra of unmodified and C6-S-S modified ASYN(1-5)}

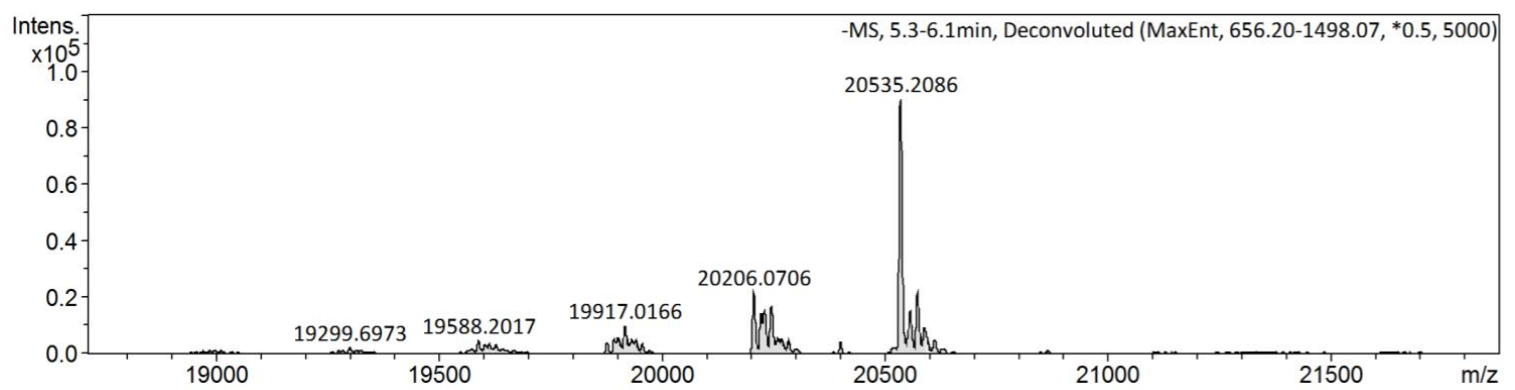

Figure 5. 6: Deconvoluted ESI-MS spectra of ASYN1.

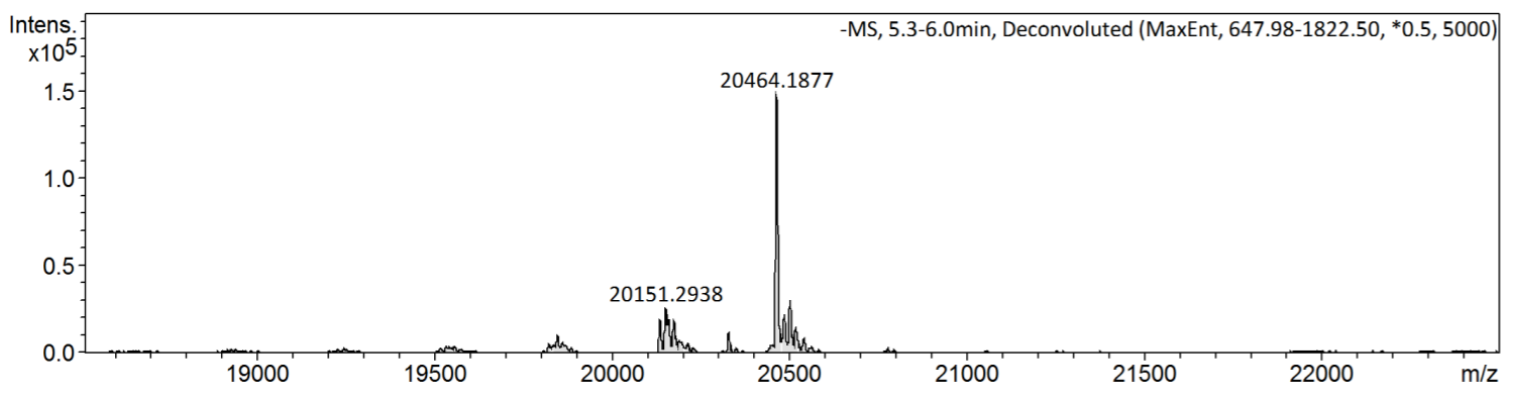

Figure 5. 7: Deconvoluted ESI-MS spectra of ASYN2.

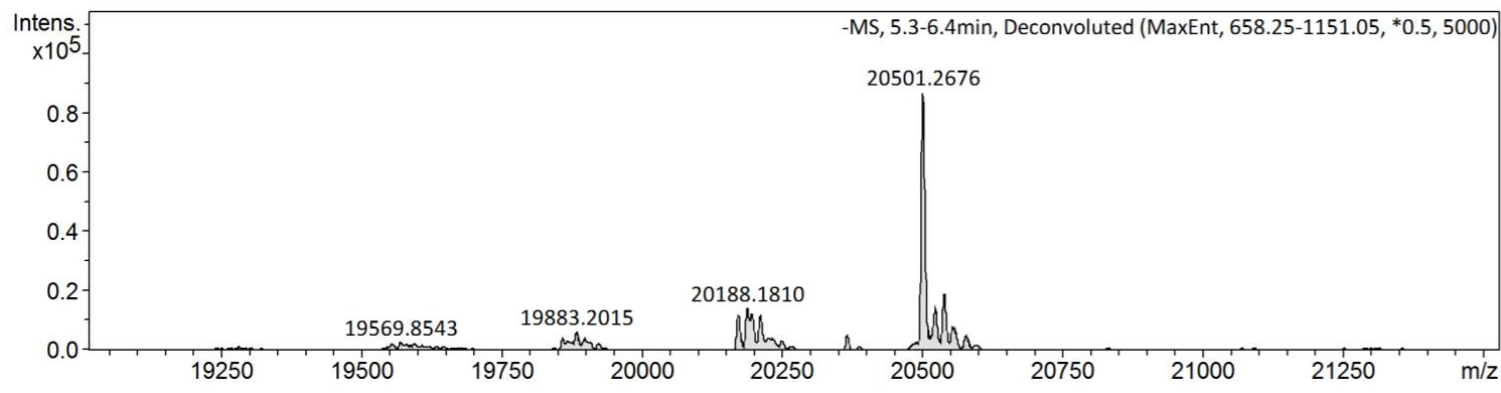

Figure 5. 8: Deconvoluted ESI-MS spectra of ASYN3. 


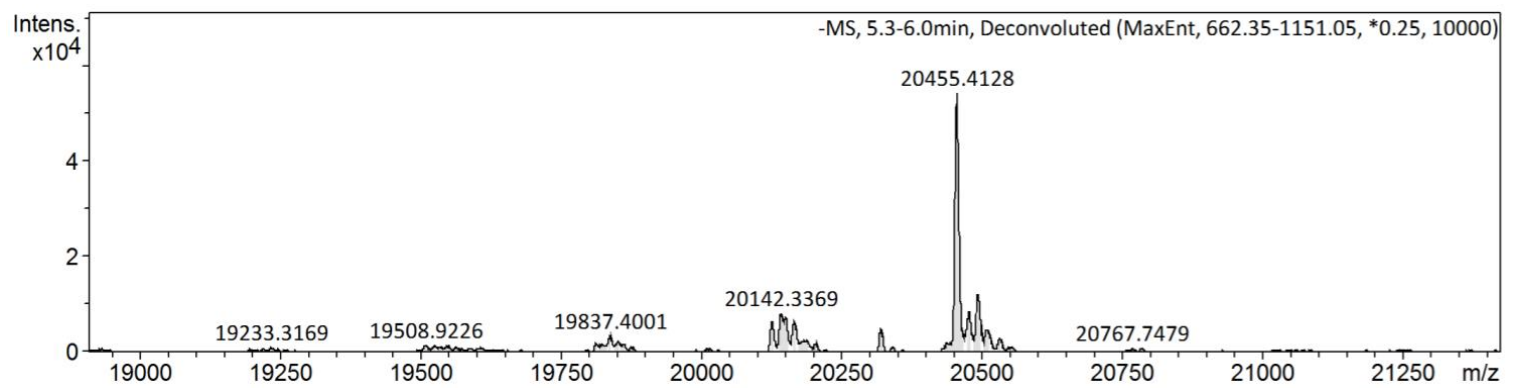

Figure 5. 9: Deconvoluted ESI-MS spectra of ASYN4.

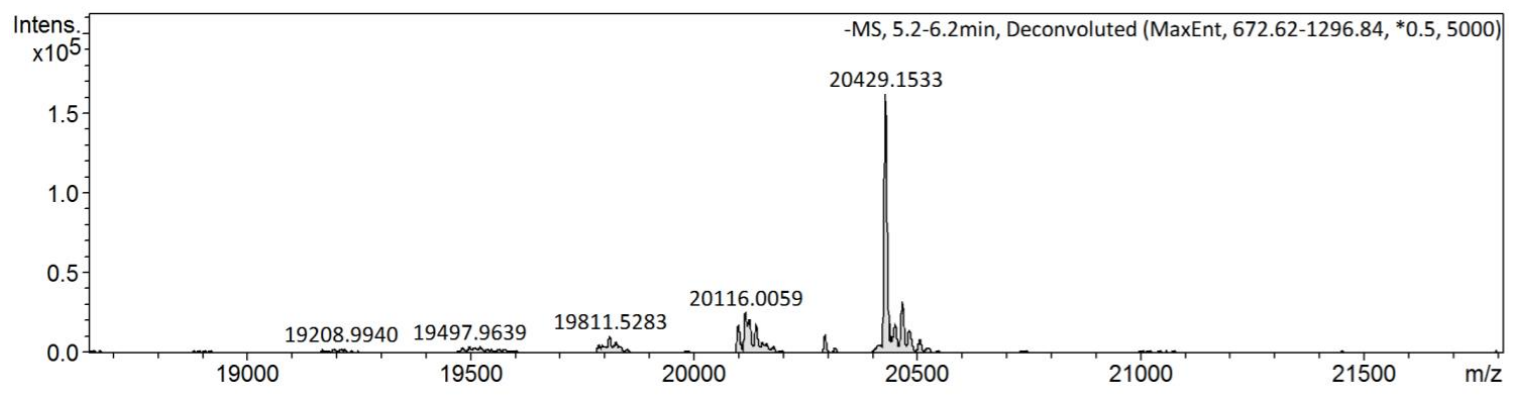

Figure 5. 10: Deconvoluted ESI-MS spectra of ASYN5.

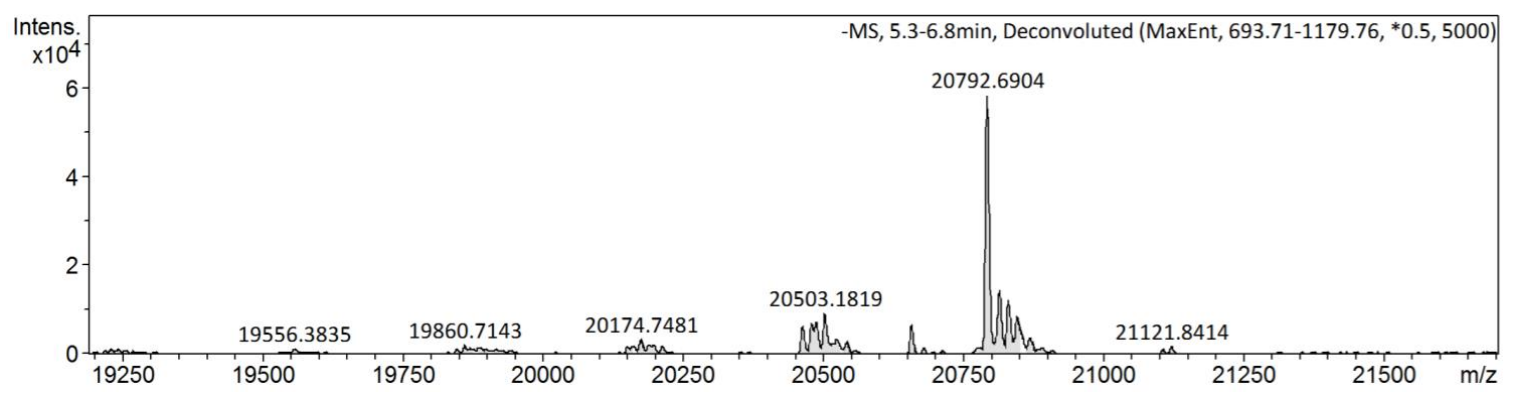

Figure 5. 11: Deconvoluted ESI-MS spectra of A2TMR. 


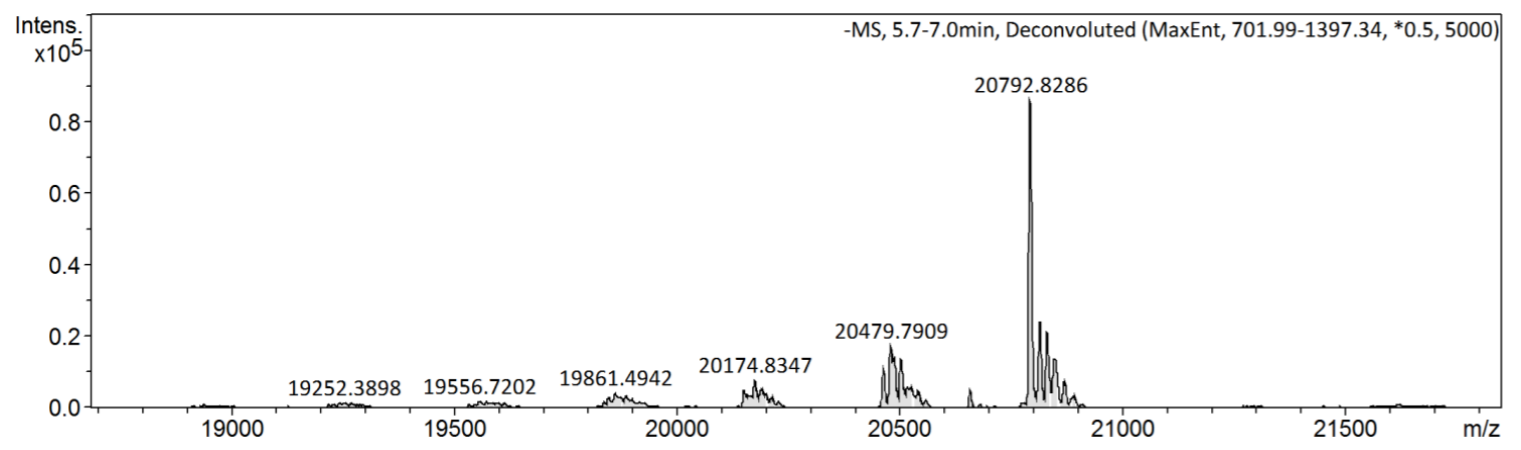

Figure 5. 12: Deconvoluted ESI-MS spectra of ASYN2-TM.

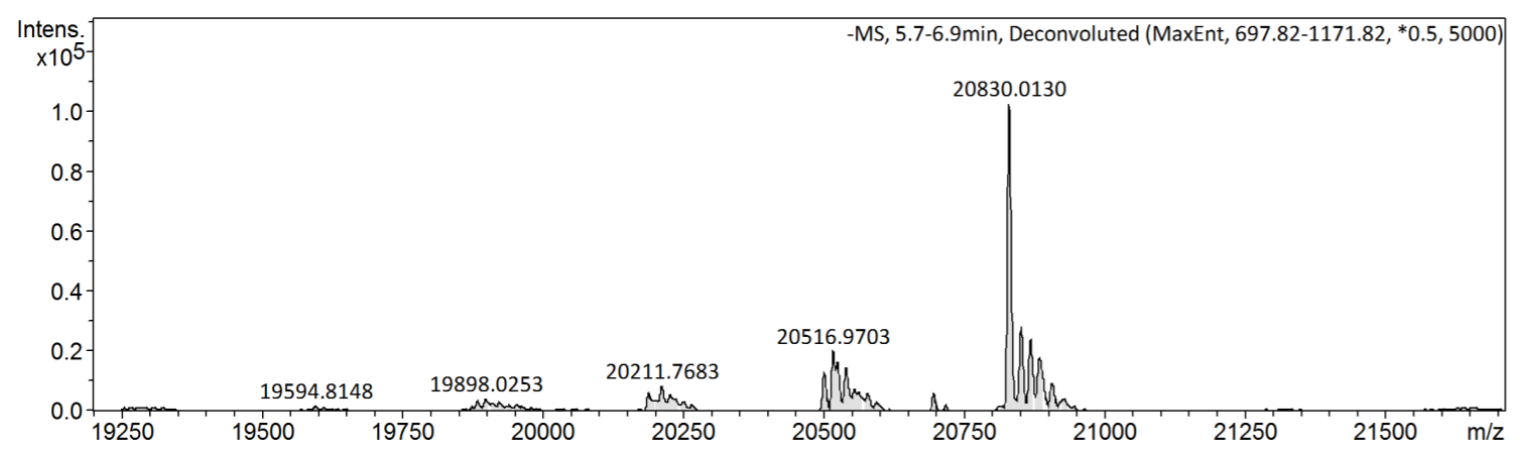

Figure 5. 13: Deconvoluted ESI-MS spectra of ASYN3-TM.

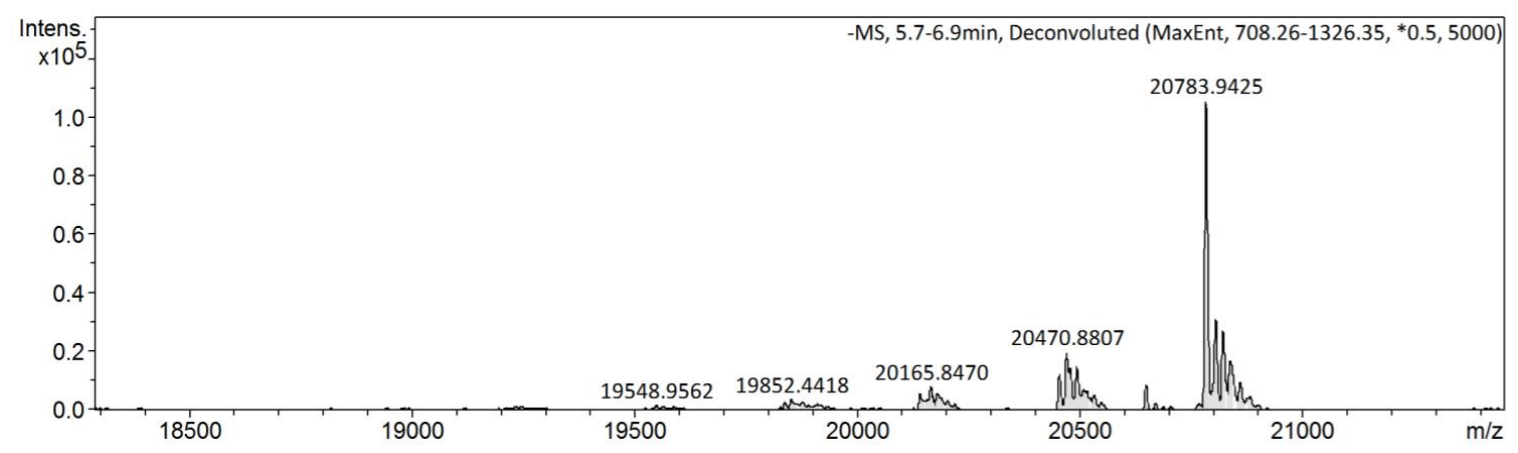

Figure 5. 14: Deconvoluted ESI-MS spectra of ASYN4-TM. 


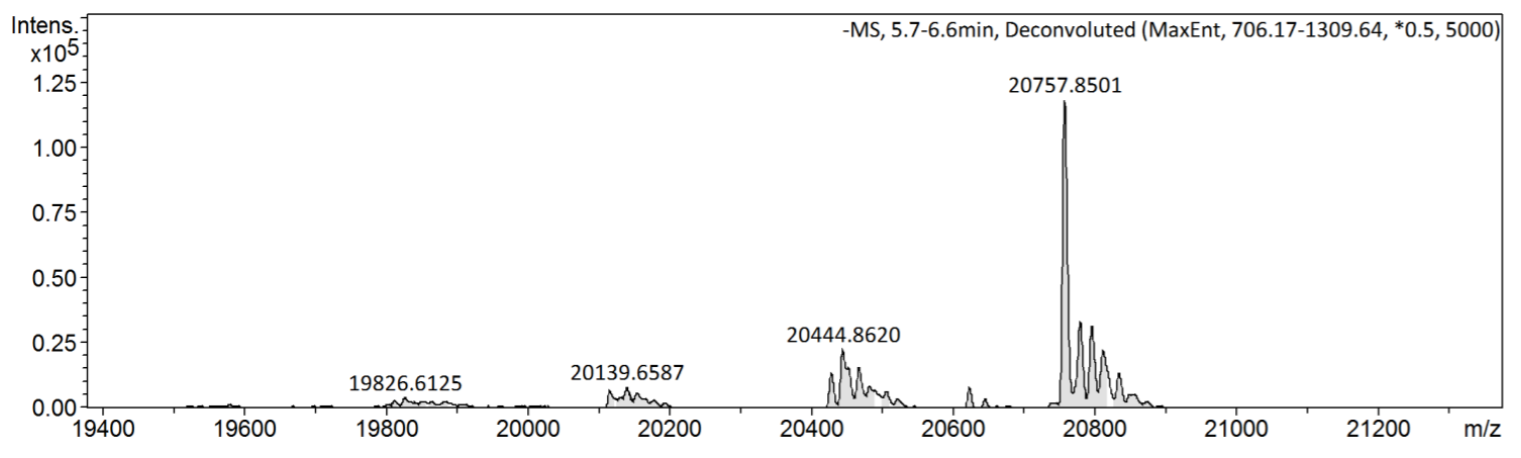

Figure 5. 15: Deconvoluted ESI-MS spectra of ASYN5-TM. 


\section{References}

(1) Fantani, J.; Yahi, N. Parkinson's Disease. In Brain Lipids in Synaptic Function and Neurological Disease: Clues to Innovative Therapeutic Strategies for Brain Disorders; Elsevier Science \& Technology: London, 2015; pp 223-251.

(2) Pfeiffer, R. F.; Wszolek, Z. K.; Ebadi, M. Parkinson's Disease, 2nd ed.; CRC Press Inc.: London, 2012.

(3) Esposito, L. A. Top Med. Chem. 2016, 18, 43-110.

(4) Fahn, S. Ann. N.Y. Acad. Sci. 2003, 991, 1-14.

(5) Larson, M. E.; Sherman, M. A.; Greimel, S.; Kuskowski, M.; Schneider, J. A.; Bennett, D. A.; Lesné, S. E. J. Neurosci. 2012, 32 (30), 10253-10266.

(6) Goedert, M. Nat. Rev. Neurosci. 2001, 2, 492-501.

(7) Stefanis, L. Cold Spring Harb. Perspect. Med. 2012, 2 (2), 1-23.

(8) Hurtig, H.I.; Trojanowski, J.Q.; Galvin, J.; Ewbank, D.; Schmidt, M.L.; Lee, V.M.Y.; Clark, C.M.; Glosser, G.; Stern, M.B.; Gollomp, S.M.; Arnold, S. E. Neurology. 2000, 54 (10), 1916-1921.

(9) Lashuel, H. A.; Petre, B. M.; Wall, J.; Simon, M.; Nowak, R. J.; Walz, T.; Lansbury, P. T. J. Mol. Biol. 2002, 322 (5), 1089-1102.

(10) Breydo, L.; Wu, J. W.; Uversky, V. N. Biochim. Biophys. Acta. 2012, 1822, 261285.

(11) Bendor, J. T.; Logan, T. P.; Edwards, R. H. Neuron. 2013, 79 (6), 1044-1066.

(12) Uversky, V. N.; Li, J.; Fink, A. L. J. Biol. Chem. 2001, 276 (14), 10737-10744.

(13) Li, J.; Uversky, V. N.; Fink, A. L. Biochemistry. 2001, 40 (38), 11604-11613.

(14) Fauvet, B.; Mbefo, M. K.; Fares, M. B.; Desobry, C.; Michael, S.; Ardah, M. T.; Tsika, E.; Coune, P.; Prudent, M.; Lion, N. J. Biol. Chem. 2012, 287 (19), 1534515364.

(15) Bartels, T.; Choi, J. G.; Selkoe, D. J. Nature. 2011, 477 (7362), 107-111.

(16) Wang, W.; Perovic, I.; Chittuluru, J.; Kaganovich, A.; Nguyen, L. T. T.; Liao, J.; Auclair, J. R.; Johnson, D.; Landeru, A.; Simorellis, A. K. Proc. Natl. Acad. Sci. U. S. A. 2011, 108 (43), 17797-17802.

(17) Ottolini, D.; Calí, T.; Szabò, I.; Brini, M. Biol. Chem. 2017, 398 (1), 77-100.

(18) Fantini, J.; Carlus, D.; Yahi, N. Biochim. Biophys. Acta-Biomembr. 2011, 1808 (10), 2343-2351.

(19) Sato, H.; Kato, T.; Arawaka, S. Rev. Neurosci. 2013, 24 (2), 115-123.

(20) Bertoncini, C. W.; Jung, Y. S.; Fernandez, C. O.; Hoyer, W.; Griesinger, C.; Jovin, T. M.; Zweckstetter, M. Proc. Natl. Acad. Sci. U. S. A. 2005, 102 (5), 1430-1435.

(21) George, J. M. Genome Biol. 2001, 3 (1), 3002.1-3002.6.

(22) Marques, O.; Outeiro, T. F. Cell Death Dis. 2012, 3, e350.

(23) Kaczmer, A.W-.; Gandhi, S.; Wood, N. W. TRENDS Mol. Med. 2006, 12 (11), 521-528.

(24) Lashuel, H.A.; Overk, C.R.; Oueslati, A.; Masliah, E. Nat. Rev. Neurosci. 2013, $14,38-48$.

(25) Lang, T.; Jahn, R. Pharmacology of Neurotransmitter Release. In Handbook of Experimental Pharmacology Vol 184; Springer-Verlag, 2008; pp 107-127.

(26) Tofaris, G.K.; Razzaq, A.; Ghetti, B.; Lilley, K.S.; Spillantini, M. G. J. Biol. Chem. 2003, 278, 44405-44411. 
(27) Cabin, D. E.; Shimazu, K.; Murphy, D.; Cole, N. B.; Gottschalk, W.; McIlwain, K. L.; Orrison, B.; Chen, A.; Ellis, C. E.; Paylor, R. J. Neurosci. 2002, 22 (20), 87978807.

(28) Chandra, S.; Fornai, F.; Kwon, H.; Yazdani, U.; Atasoy, D.; Liu, X.; Hammer, R. E.; Battaglia, G.; German, D. C.; Castillo, P. E. Pnas. 2004, 101 (41), 1496614971.

(29) Beyer, K. a-Synuclein Structure, Acta Neuropathol. 2006, 112 (3), 237-251.

(30) Fujiwara, H.; Hasegawa, M.; Dohmae, N.; Kawashima, A.; Masliah, E.; Goldberg, M. S.; Shen, J.; Takio, K.; Iwatsubo, T. Nat. Cell Biol. 2002, 4 (2), 160-164.

(31) Nishe, M.; Mori, F.; Fujiwara, H.; Hasegawa, M.; Yoshimoto, M.; Iwatsubo, T.; Takahashi, H.; Wakabayashi, K. Acta Neuropathol. 2004, 107 (4), 292-298.

(32) Smith, W. W.; Margolis, R. L.; Li, X.; Troncoso, J. C.; Lee, M. K.; Dawson, V. L.; Dawson, T. M.; Iwatsubo, T.; Ross, C. A. J. Neurosci. 2005, 25 (23), 5544-5552.

(33) Lee, K. W.; Chen, W.; Junn, E.; Im, J. Y.; Grosso, H.; Sonsalla, P. K.; Feng, X.; Ray, N.; Fernandez, J. R.; Chao, Y. J. Neurosci. 2011, 31 (19), 6963-6971.

(34) Luk, K. C.; Song, C.; O’Brien, P.; Stieber, A.; Branch, J. R.; Brunden, K. R.; Trojanowski, J. Q.; Lee, V. M. Y. Proc. Natl. Acad. Sci. U. S. A. 2009, 106 (47), 20051-20056.

(35) Volpicelli-Daley, L. A.; Luk, K. C.; Patel, T. P.; Tanik, S. A.; Riddle, D. M.; Stieber, A.; Meaney, D. F.; Trojanowski, J. Q.; Lee, V. M. Y. Neuron 2011, 72 (1), 57-71.

(36) Volpicelli-Daley, L. A.; Luk, K. C.; Lee, V. M. Y. Nat. Protoc. 2014, 9 (9), 21352146.

(37) Tarutani, A.; Suzuki, G.; Shimozawa, A.; Nonaka, T.; Akiyama, H.; Hisanaga, S. I.; Hasegawa, M. J. Biol. Chem. 2016, 291 (36), 18675-18688.

(38) Danzer, K. M.; Krebs, S. K.; Wolff, M.; Birk, G.; Hengerer, B. J. Neurochem. 2009, 111 (1), 192-203.

(39) Obeso, J. A.; Rodriguez-Oroz, M. C.; Goetz, C. G.; Marin, C.; Kordower, J. H.; Rodriguez, M.; Hirsch, E. C.; Farrer, M.; Schapira, A. H. V.; Halliday, G. Nat. Med. 2010, 16 (6), 653-661.

(40) Chen, L.; Feany, M. B. Nat. Neurosci. 2005, 8 (5), 657-663.

(41) Hansen, C.; Li, J.-Y. TRENDS Mol. Med. 2012, 18 (5), 248-255.

(42) Jang, A.; Lee, H. J.; Suk, J. E.; Jung, J. W.; Kim, K. P.; Lee, S. J. J. Neurochem. 2010, 113 (5), 1263-1274.

(43) Cookson, M. R. Mol. Neurodegen. 2009, 4 (9), 1-14.

(44) Dawson, T.M.; Dawson, V. L. Science. 2003, 302, 819-822.

(45) Lucking, C.B.; Brice, A. C. Cell. Mol. Life Sci. 2000, 57, 1894-1908.

(46) Mazzulli, J. R.; Mishizen, A. J.; Giasson, B. I.; Lynch, D. R.; Thomas, S. A.; Nakashima, A.; Nagatsu, T.; Ota, A.; Ischiropoulos, H. J. Neurosci. 2006, 26 (39), $10068-10078$.

(47) Conway, K. A.; Rochet, J. C.; Bieganski, R. M.; Lansbury, J. Science. 2001, 294 (5545), 1346-1349.

(48) Hitti, F.L.; Ramayya, A.G.; McShane, B.J.; Yang, A.I.; Vaughan, K.A.; Baltuch, G. H. J. Neurosurg. 2019, 132 (1).

(49) Fahn, S.; Oake, D.; Shoulson, I.; Kieburtz, K.; Rudolph, A.; Lang, A. N. Engl. J. Med. 2004, 351 (24), 2498-2508. 
(50) Davie, C. A. Br. Med. Bull. 2008, 86, 109-127.

(51) Edmondson, D.E.; Mattevi, A.; Binda, C.; Li, M.; Hubalek, F. Curr. Med. Chem. 2004, 11 (15), 1983-1993.

(52) Watson, J.D.; Crick, F. H. C. Nature. 1953, 3 (171), 737-738.

(53) Klug, A. Nature. 1968, 219 (5156), 808-844.

(54) Tuerk, C.; Gold, L. Science. 1990, 249 (4968), 505-510.

(55) Jayasena, S. D. Clin. Chem. 1999, 45 (9), 1628-1650.

(56) Dollins, C. M.; Nair, S.; Sullenger, B. A. Hum. Gene Ther. 2008, 19 (5), 443-450.

(57) Song, S.; Wang, L.; Li, J.; Fan, C.; Zhao, J. TrAC - Trends Anal. Chem. 2008, 27 (2), 108-117.

(58) Song, K. M.; Lee, S.; Ban, C. Sensors 2012, 12 (1), 612-631.

(59) Tombelli, S.; Minunni, M.; Mascini, M. Biosens. Bioelectron. 2005, 20 (12), 2424-2434.

(60) McGinely, N. L.; Plumb, J. A.; Wheate, N. J. J. Inorg. Biochem. 2013, 128, 124 130.

(61) Brody, E. N.; Gold, L. Rev. Mol. Biotechnol. 2000, 74 (1), 5-13.

(62) Fan, L.; Zhao, G.; Shi, H.; Liu, M.; Li, Z. Biosens. Bioelectron. 2013, 43, 15, $12-$ 18.

(63) Ellington, A. D.; Szostak, J. W. Nature. 1990, 346 (6287), 818-822.

(64) Darmostuk, M.; Rimpelova, S.; Gbelcova, H.; Ruml, T. Biotechnol. Adv. 2014, 33 (6), 1141-1161.

(65) Jing, Meng; Bowser, M. T. Anal. Chim. Acta. 2011, 686, 1-25.

(66) Kastritis, P. L.; Bonvin, A. M. J. J. J. R. Soc. Interface. 2013, 10 (79), 1-27.

(67) Rippe, K. BIF Futur. 1997, 20-26.

(68) Wilson, B. D.; Soh, H. T. Re-Evaluating the Conventional Wisdom about Binding Assays. Trends Biochem. Sci. 2020, 1-11.

(69) Levy, Y.; Onuchic, J. N. Annu. Rev. Biophys. Biomol. Struct. 2006, 35 (1), 389415.

(70) Reddy, G.; Straub, J. E.; Thirumalai, D. Proc. Natl. Acad. Sci. U. S. A. 2010, 107 (50), 21459-21464.

(71) Ellington, A.; Pollard, J. D. Curr. Protoc. Nucleic Acid Chem. 2000, 1-22.

(72) Guidebook for the Synthesis of Oligonucleotides; Link Technologies Ltd.: United Kingdom, 2015; pp 24-31.

(73) Caruthers, M. H. Biochem. Soc. Trans. 2011, 39 (2), 575-580.

(74) Reese, C. B. Org. Biomol. Chem. 2005, 3 (21), 3851-3868.

(75) Harris, D. C. Quantitative Chemical Analysis, 8th ed.; W.H. Freeman and Company: New York, 2010.

(76) Brett, C.M.A.; Brett, A. M. O. Electrochemistry: Principles, Methods, and Applications; Oxford University Press, 1993.

(77) Biesheuvel, P. M.; Dykstra, J. E. Chem. Phys. 2018, 1-10.

(78) Bard, A.J.; Faulkner, L. R. Electrochemical Methods: Fundamentals and Applications, 2nd ed.; John Wiley \& Sons Inc., 2001.

(79) Lim, Y. C.; Kouzani, A. Z.; Duan, W. J. Biomed. Nanotechnol. 2010, 6 (2), 93105.

(80) Xu, Y.; Cheng, G.; He, P.; Fang, Y. Electroanalysis 2009, 21 (11), 1251-1259.

(81) Han, K.; Liang, Z.; Zhou, N. Sensors. 2010, 10 (5), 4541-4557. 
(82) Byfield, M. P.; Abuknesha, R. A. Biosens. Bioelectron. 1994, 9 (4-5), 373-399.

(83) Sethi, R. S. Biosens. Bioelectron. 1994, 9 (3), 243-264.

(84) Sassolas, A.; Blum, L. J.; Béatrice, D. L. B. Electroanalysis 2009, 21 (11), 1237 1250.

(85) Feng, C.; Dai, S.; Wang, L. Biosens. Bioelectron. 2014, 59, 64-74.

(86) Qureshi, A.; Gurbuz, Y.; Niazi, J. H. Sensors Actuators, B Chem. 2012, 171-172, 62-76.

(87) Bruckner-Lea, C. J. Electrochem. Soc. Interface 2004, 13 (2), 36-42.

(88) Wang, J.; Rivas, G.; Cai, X.; Palecek, E.; Nielson, P.; Shiraishi, H.; Dontha, N.; Luo, D.; Parrado, C.; Chicharro, M.; Farias, P.A.M.; Valera, F.S.; Grant, D.H.; Ozsoz, M.; Flair, M. N. Anal. Chim. Acta 1997, 347, 1-8.

(89) Compton, R.G.; Banks, C. E. Understanding Voltammetry, 2nd ed.; Imperial College Press: London, 2011.

(90) Bond, A. M. Electrochemistry: General Introduction. In Comprehensive Coordination Chemistry II; McCleverty, J.A.; Meyer, T. J., Ed.; Oxford, 2003; pp 197-222.

(91) Dong, Y. Aptamers for Analytical Applications; Wiley VCH: Weinheim, Germany, 2018.

(92) Liu, J. Phys. Chem. Chem. Phys. 2012, 14 (30), 10485-10496.

(93) Sassolas, A.; Leca-Bouvier, B. D.; Blum, L. J. Chem. Rev. 2008, 108 (1), 109-139.

(94) Giese, B.; McNaughton, D. J. Phys. Chem. B 2002, 106 (1), 101-112.

(95) Chen, Q.; Frankel, D. J.; Richardson, N. V. Langmuir. 2002, 18 (8), 3219-3225.

(96) Kimura-Suda, H.; Petrovykh, D. Y.; Tarlov, M. J.; Whitman, L. J. J. Am. Chem. Soc. 2003, 125 (30), 9014-9015.

(97) Frasconi, M.; Mazzei, F.; Ferri, T. Anal. Bioanal. Chem. 2010, 398 (4), 15451564.

(98) Xue, Y.; Li, X.; Li, H.; Zhang, W. Nat. Commun. 2014, 5, 1-9.

(99) Vericat, C.; Vela, M. E.; Benitez, G. A.; Martin Gago, J. A.; Torrelles, X.; Salvarezza, R. C. J. Phys. Condens. Matter 2006, 18 (48).

(100) Häkkinen, H. Nat. Chem. 2012, 4 (6), 443-455.

(101) Bürgi, T. Nanoscale 2015, 7 (38), 15553-15567.

(102) Pensa, E.; Cortés, E.; Corthey, G.; Carro, P.; Vericat, C.; Fonticelli, M. H.; Benítez, G.; Rubert, A. A.; Salvarezza, R. C. Acc. Chem. Res. 2012, 45 (8), 11831192.

(103) Balamurugan, S.; Obubuafo, A.; McCarley, R. L.; Soper, S. A.; Spivak, D. A. Anal. Chem. 2008, 80 (24), 9630-9634.

(104) Steed, Jonathan W.; Turner, David R.; Wallace, K. J. Core Concepts in Supramolecular Chemistry and Nanochemistry; Wiley, 2007.

(105) Love, J. C.; Estroff, L. A.; Kriebel, J. K.; Nuzzo, R. G.; Whitesides, G. M. Chem. Rev. 2005, 105 (4), 1103-1170.

(106) Fan, C.; Plaxco, K. W.; Heeger, A. J. PNAS 2003, 100 (16), 9134-9137.

(107) Xiao, Y.; Lubin, A. A.; Heeger, A. J.; Plaxco, K. W. Angew. Chem. Int. Ed. 2005, $44,5456-5459$.

(108) Taghdisi, S. M.; Danesh, N. M.; Nameghi, M. A.; Ramezani, M.; Alibolandi, M.; Hassanzadeh-Khayat, M.; Emrani, A. S.; Abnous, K. Biosens. Bioelectron. 2019, $123,14-18$. 
(109) Tsukakoshi, K.; Abe, K.; Sode, K.; Ikebukuro, K. Anal. Chem. 2012, 84 (13), 5542-5547.

(110) Zheng, D.; Zou, R.; Lou, X. Anal. Chem. 2012, 84 (8), 3554-3560.

(111) Luo, L.; Xu, F.; Shi, H.; He, X.; Qing, T.; Lei, Y.; Tang, J.; He, D.; Wang, K. Talanta. 2017, 169, 57-63.

(112) Guo, J.; Yuan, C.; Yan, Q.; Duan, Q.; Li, X.; Yi, G. Biosens. Bioelectron. 2018, 105, 103-108.

(113) Farjami, E.; Clima, L.; Gothelf, K. V.; Ferapontova, E. E. Analyst. 2010, 135 (6), 1443-1448.

(114) Barsoukov, E.; Macdonald, J. R. Impedance Spectroscopy Theory, Experiment, and Applications, 2nd ed.; Wiley Interscience: Hoboken, New Jersey, 2005.

(115) Orazem, M.E.; Tribollet, B. Electrochemical Impedance Spectroscopy; Wiley: Hoboken, New Jersey, 2008.

(116) Macdonald, J. R. Ann. Biomed. Eng. 1992, 20, 289-305.

(117) Cabuzu, D.; Cirja, A.; Puiu, R.; Grumezescu, A. M. Curr. Top. Med. Chem. 2015, 15 (16), 1605-1613.

(118) Zhou, C.; Zou, H.; Sun, C.; Ren, D.; Xiong, W.; Li, Y. Anal. Bioanal. Chem. 2018, 410 (12), 2981-2989.

(119) Vilela, D.; González, M. C.; Escarpa, A. Anal. Chim. Acta 2012, 751, 24-43.

(120) Sadrolhosseini, A. R.; Shafie, S.; Fen, Y. W. Appl. Sci. 2019, 9 (7), 1497.

(121) Maier, A. Plasmonics: Fundamentals and Applications; Springer Science and Business Media, 2007.

(122) Gordon, J. G.; Ernst, S. Surf. Sci. 1980, 101 (1-3), 499-506.

(123) Ghosh, S. K.; Nath, S.; Kundu, S.; Esumi, K.; Pal, T. J. Phys. Chem. B 2004, 108 (37), 13963-13971.

(124) Kress-Rogers, E. Biosensors and Electronic Noses for Practical Application. In Handbook of biosensors and electronic noses; CRC Press Inc., 2007; pp 3-39.

(125) Homola, J.; Piliarik, M. Ser. Chem. Sens. Biosens. 2006, 4, 45-67.

(126) Pluchery, O. Optical Properties of Gold Nanoparticles. In Gold Nanoparticles for Physics, Chemistry, and Biology; Imperial College Press: London, 2012; pp 43-73.

(127) Stewart, M. E.; Anderton, C. R.; Thompson, L. B.; Maria, J.; Gray, S. K.; Rogers, J. A.; Nuzzo, R. G. Chem. Rev. 2008, 108 (2), 494-521.

(128) Li, Y.; Jing, C.; Zhang, L.; Long, Y. T. Chem. Soc. Rev. 2012, 41 (2), 632-642.

(129) Kim, S. H.; Medeiros-Ribeiro, G.; Ohlberg, D. A. A.; Williams, R. S.; Heath, J. R. J. Phys. Chem. B 1999, 103 (47), 10341-10347.

(130) Sepúlveda, B.; Angelomé, P. C.; Lechuga, L. M.; Liz-Marzán, L. M. Nano Today. 2009, 4 (3), 244-251.

(131) Wang, P.; Wan, Y.; Ali, A.; Deng, S.; Su, Y.; Fan, C.; Yang, S. Sci. China Chem. 2016, 59 (2), 237-242.

(132) Gu, Man Bock; Kim, H.-S. Biosensors Based on Aptamers and Enzymes; SpringerVerlag: Berlin, 2014.

(133) Cleaver, J.E.; Crowley, E. Front. Biosci. 2002, 7, 1024-1043.

(134) Lampman, G.M.; Pavia, D.L.; Kriz, G.S.; Vyvyan, J. R. Spectroscopy; Cengage Learning Products, 2010.

(135) Brown, E.; Pysh, E. S. J. Chem. Phys. 1972, 56 (1), 31-37.

(136) Mergny, J-L.; Lacroix, L. Oligonucleotides. 2004, 13 (6), 515-537. 
(137) Greenfield, Norma, J. Nat. Protoc. 2006, 1 (6), 2876-2890.

(138) Ranjbar, B.; Gill, P. Chem. Biol. Drug Des. 2009, 74 (2), 101-120.

(139) Kelly, S. M.; Price, N. C. Curr. Protein Pept. Sci. 2000, 1 (4), 349-386.

(140) Johnson Jr., W. C. Determination of the Conformation of Nucleic Acids by Electronic CD. In Circular Dichroism and the Conformational Analysis of Biomolecules; Fasman, G. D., Ed.; Springer Science and Business Media: New York, 1996; pp 433-438.

(141) Kypr, J.; Kejnovská, I.; Renčiuk, D.; Vorlíčková, M. Nucleic Acids Res. 2009, 37 (6), 1713-1725.

(142) Vorlíčková, Michaela; Kejnovská, Iva; Bednárová, Klára; Renčiuk, Daniel; Kypr, J. Chirality 2012, 24, 691-698.

(143) Ivanov, V. I.; Minchenkova, L. E.; Minyat, E. E.; Frank-Kamenetskii, M. D.; Schyolkina, A. K. J. Mol. Biol. 1974.

(144) Gray, D.M.; Wen, J.D.; Gray, C.W.; Repges, R.; Repges, C.; Raabe, G.; Fleischhauer, J. Chirality. 2008, 20, 431-440.

(145) Shukla, M. K.; Leszczynski, J. J. Biomol. Struct. Dyn. 2007, 25 (1), 93-118.

(146) Tsukakoshi, K.; Harada, R.; Sode, K.; Ikebukuro, K. Biotechnol. Lett. 2010, 32 (5), 643-648.

(147) Zheng, Y.; Qu, J.; Xue, F.; Zheng, Y.; Yang, B.; Chang, Y.; Yang, H.; Zhang, J. Mol. Ther. Nucleic Acids 2018, 11, 228-242.

(148) Callahan, J. P. The Selection of DNA Aptamers for the Prevention of AlphaSynuclein Aggregation as a Therapeutic Tool in Parkinson's Disease, Carleton University, 2017.

(149) Hunt, V. Characterization and Delivery of DNA Aptamers Selected for the Prevention of Alpha-Synuclein Aggregation in Parkinson's Disease, Carleton University, 2018.

(150) Bellaousov, S.; Reuter, J. S.; Seetin, M. G.; Mathews, D. H. Nucleic Acids Res. 2013, 41

(151) Kikin, O.; D’Antonio, L.; Bagga, P. S. Nucleic Acids Res. 2006, 34, 676-682.

(152) Neidle, S. Therapeutic Applications of Quadruplex Nucleic Acids, 1st ed.; Elsevier Inc.: London, 2012.

(153) Burge, S.; Parkinson, G. N.; Hazel, P.; Todd, A. K.; Neidle, S. Nucleic Acids Res. 2006, 34 (19), 5402-5415.

(154) Cao, Q.; Li, Y.; Freisinger, E.; Qin, P. Z.; Sigel, R. K. O.; Mao, Z. W. G- Inorg. Chem. Front. 2017, 4, 10-32.

(155) Kopylov, m.; Jackson, T. M.; Stroupe, M. E. Molecules. 2019, 24 (10), 1988.

(156) Xu, S.; Chen, X.; Peng, G.; Jiang, L.; Huang, H. Anal. Bioanal. Chem. 2018, 410 (23), 5879-5887.

(157) Ocaña, C.; Pacios, M.; del Valle, M. Sensors. 2012, 12 (3), 3037-3048.

(158) Ulman, A. Chem. Rev. 1996, 96 (4), 1533-1554.

(159) Elgrishi, N.; Rountree, K. J.; McCarthy, B. D.; Rountree, E. S.; Eisenhart, T. T.; Dempsey, J. L. J. Chem. Educ. 2018, 95 (2), 197-206.

(160) McCreery, R. L. Chem. Rev. 2008, 108 (7), 2646-2687.

(161) Monteriro, M. C. O.; Koper, M. T. M. Electrochim. Acta 2019, 325 (1), 134915.

(162) Bhalla, V.; Carrara, S.; Stagni, C.; Samori, B. Thin Solid Films 2010, 518, 33603366. 
(163) Hoare, J. P. J. Electrochem. Soc. 1984, 131 (8), 1808-1815.

(164) Angerstein-Kozlowska, H.; Conway, B. E.; Hamelin, A.; Stoicoviciu, L. Electrochim. Acta 1986, 31 (8), 1051-1061.

(165) Laitinen, H. A.; Chao, M. S. J. Electrochem. Soc. 1961, 108 (8), 726.

(166) Brumme, S. B.; Makrides, A. C. J. Electrochem. Soc. 1964, 111 (10), 1122-1128.

(167) Zhang, W.; Bas, A. D.; Ghali, E.; Choi, Y.. Trans. Nonferrous Met. Soc. China English Ed. 2015, 25 (6), 2037-2046.

(168) Wakkad, S.E.S.E.; Din, A.M.S.E. The Andoic Oxidation of Metals at Very Low Current Density. 1954, 3098-3102.

(169) Du, P.; Liu, S.; Wu, P.; Cai, C. Electrochim. Acta 2007, 52 (23), 6534-6547.

(170) Dickinson, E. J. F.; Limon-petersen, J. G.; Rees, N. V; Compton, R. G. J. Phys. Chem. 2009, 11157-11171.

(171) Goldstein, E. L.; Van de Mark, M. R. Electrochim. Acta. 1982, 27 (8), 1079-1085.

(172) Sekar, N.; Ramasamy, R. P. J. Microb. Biochem. Technol. 2013, 5, s6-004.

(173) Steel, A. B.; Herne, T. M.; Tarlov, M. J. Anal. Chem. 1998, 70 (22), 4670-4677.

(174) Bai, L.; Yuan, R.; Chai, Y.; Yuan, Y.; Wang, Y.; Xie, S. Chem. Commun. 2012, 48 (89), 10972-10974.

(175) Konopka, S. J.; McDuffie, B. Anal. Chem. 1970, 42 (14), 1741-1746.

(176) Getz, E. B.; Xiao, M.; Chakrabarty, T.; Cooke, R.; Selvin, P. R. Anal. Biochem. 1999, 273 (1), 73-80.

(177) Burns, J. A.; Butler, J. C.; Moran, J.; Whitesides, G. M. J. Org. Chem. 1991, 56 (8), 2648-2650.

(178) Peterson, A. W. Nucleic Acids Res. 2001, 29 (24), 5163-5168.

(179) Wu, J.; Campuzano, S.; Halford, C.; Haake, D. A.; Wang, J. Anal. Chem. 2010, 82 (21), 8830-8837.

(180) Boozer, C.; Chen, S.; Jiang, S. Langmuir. 2006, 22 (10), 4694-4698.

(181) Keighley, S. D.; Li, P.; Estrela, P.; Migliorato, P. Biosens. Bioelectron. 2008, 23 (8), 1291-1297.

(182) Lao, R.; Song, S.; Wu, H.; Wang, L.; Zhang, Z.; He, L.; Fan, C. Anal. Chem. 2005, 77 (19), 6475-6480.

(183) Herne, T. M.; Tarlov, M. J. J. Am. Chem. Soc. 1997, 119 (38), 8916-8920.

(184) Steel, A. B.; Levicky, R. L.; Herne, T. M.; Tarlov, M. J. Biophys. J. 2000, 79 (2), 975-981.

(185) Anastassopoulou, J.; Theophanides, T. Crit. Rev. Oncol. Hematol. 2002, 42 (1), 79-91.

(186) Reuss, A. J.; Vogel, M.; Weigand, J. E.; Suess, B.; Wachtveitl, J. Biophys. J. 2014, 107 (12), 2962-2971.

(187) Furst, A. L.; Hill, M. G.; Barton, J. K. Langmuir. 2013, 29 (52), 16141-16149.

(188) Su, L.; Sankar, C. G.; Sen, D.; Yu, H. Z. Anal. Chem. 2004, 76 (19), 5953-5959.

(189) Meade, R. M.; Fairlie, D. P.; Mason, J. M. Mol. Neurodegener. 2019, 14 (1), 1-14.

(190) Fjorback, W. A.; Varming, K.; Jensen, H. P. Scand. J. Clin. Lab. Invest. 2006, 67 (4), 431-435.

(191) Viodé, A.; Epelbaum, S.; Benyounes, I.; Verny, M.; Dubois, B.; Junot, C.; Fenaille, F.; Lamari, F.; Becher, F. Analyst 2019, 144 (21), 6342-6351.

(192) Wang, Y.; Shi, M.; Chung, K. A.; Zabetian, C. P.; Leverenz, J. B.; Berg, D.; Srulijes, K.; Trojanowski, J. Q.; Lee, V. M.; Andrew, D. Science Translational 
Med. 2012, 4 (121), 1-15.

(193) Oeckl, P.; Metzger, F.; Nagl, M.; Von Arnim, C. A. F.; Halbgebauer, S.; Steinacker, P.; Ludolph, A. C.; Otto, M. Mol. Cell. Proteomics 2016, 15 (10), 3126-3138.

(194) Bogomolova, A.; Komarova, E.; Reber, K.; Gerasimov, T.; Yavuz, O.; Bhatt, S.; Aldissi, M. Anal. Chem. 2009, 81 (10), 3944-3949.

(195) McKeague, M.; De Girolamo, A.; Valenzano, S.; Pascale, M.; Ruscito, A.; Velu, R.; Frost, N. R.; Hill, K.; Smith, M.; McConnell, E. M., DeRosa, M. C. Anal. Chem. 2015, 87 (17), 8608-8612.

(196) Seidel, S. A. I.; Dijkman, P. M.; Lea, W. A.; van den Bogaart, G.; JerabekWillemsen, M.; Lazic, A.; Joseph, J. S.; Srinivasan, P.; Baaske, P.; Simeonov, A. Methods. 2013, 59 (3), 301-315.

(197) Duhr, S.; Braun, D. Proc. Natl. Acad. Sci. U. S. A. 2006, 103 (52), 19678-19682.

(198) Jerabek-Willemsen, M.; André, T.; Wanner, R.; Roth, H. M.; Duhr, S.; Baaske, P.; Breitsprecher, D. J. Mol. Struct. 2014, 1077, 101-113.

(199) Jerabek-Willemsen, M.; Wienken, C. J.; Braun, D.; Baaske, P.; Duhr, S. Assay Drug Dev. Technol. 2011, 9 (4), 342-353.

(200) Wienken, C. J.; Baaske, P.; Rothbauer, U.; Braun, D.; Duhr, S. Nat. Commun. 2010, 1 (7).

(201) Reineck, P.; Wienken, C. J.; Braun, D. Electrophoresis. 2010, 31, 279-286.

(202) Sass, S.; Stöcklein, W. F. M.; Klevesath, A.; Hurpin, J.; Menger, M.; Hille, C. Analyst. 2019, 144 (20), 6064-6073.

(203) Rainard, J. M.; Pandarakalam, G. C.; McElroy, S. P. Eur. Lead Fact. Perspect. 2018, 23 (3), 225-241.

(204) Scheuermann, T. H.; Padrick, S. B.; Gardner, K. H.; Brautigam, C. A. Anal. Biochem. 2016, 496, 79-93.

(205) Malgowska, M.; Gudanis, D.; Teubert, A.; Dominiak, G.; Gdanie, Z. Biotechnologia. 2012, 93 (4), 381-390.

(206) Viglasky, V.; Hianik, T. Gen. Physiol. Biophys. 2013, 32, 149-172.

(207) Tong, X.; Lan, W.; Zhang, X.; Wu, H.; Liu, M.; Cao, C. Nucleic Acids Res. 2011, 39, 6753-6763.

(208) Lin, P. H.; Chen, R. H.; Lee, C. H.; Chang, Y.; Chen, C. S.; Chen, W. Y. Colloids Surfaces B Biointerfaces. 2011, 88 (2), 552-558.

(209) Liu, X.; Atwater, M.; Wang, J.; Huo, Q. Colloids Surfaces B Biointerfaces 2007, $58(1), 3-7$.

(210) Yang, C.; Wang, Y.; Marty, J-L. Yang, X. Biosens. Bioelectron. 2011, 26, 2724 2727.

(211) Zhang, X.; Servos, M.R.; Liu, J. Langmuir 2012, 28 (8), 3896-3902.

(212) Sang, F.; Zhang, X.; Liu, J.; Yin, S.; Zhang, Z. Spectrochim. Acta Part A Mol. Biomol. Spectrosc. 2019, 217, 122-127.

(213) Chen, Z.; Tan, Y.; Zhang, C.; Yin, L.; Ma, H.; Ye, N.; Qiang, H.; Lin, Y. Biosens. Bioelectron. 2014, 56, 46-50.

(214) Sang, F.; Liu, J.; Zhang, X.; Pan, J. Microchim. Acta 2018, 185, 267.

(215) Shayesteh, O. H.; Ghavami, R. Spectrochim. Acta Part A Mol. Biomol. Spectrosc. 2020, 226, 117644.

(216) Yao, X.; Ma, X.; Ding, C.; Jia, L. Microchim. Acta 2016, 183, 2353-2359. 
(217) He, L.; Luo, Y.; Zhi, W.; Zhou, P. Food Anal. Methods 2013, 6, 1704-1711.

(218) Zhang, S.; Wang, K.; Li, J.; Li, Z.; Sun, T. RSC Adv. 2015, 5, 75746-75752.

(219) Bala, R.; Kumar, M.; Bansal, K.; Sharma, R. K.; Wangoo, N. Biosens. Bioelectron. 2016, 85, 445-449.

(220) Tian, L.; Chang, A.; Melancon, M. P. Transl. Cancer Res. 2017, 309-312.

(221) Lynch, I.; Dawson, K. A. Protein-Nanoparticle Interactions. Nanotoday 2008, 3 (1), 40-47.

(222) Wang, P.; Wang, X.; Wang, L.; Hou, X.; Liu, W.; Chen, C. Sci. Technol. Adv. Mater. 2015, 16 (3).

(223) Wang, A.; Vangala, K.; Vo, T.; Zhang, D.; Fitzkee, N. C. J. Phys. Chem. C 2014, 118 (15), 8134-8142.

(224) Rani, M.; Moudgil, L.; Singh, B.; Kaushal, A.; Mittal, A.; Saini, G. S. S.; Tripathi, S. K.; Singh, G.; Kaura, A. RSC Adv. 2016, 6, 17373-17383.

(225) Ding, F.; Radic, S.; Chen, R.; Chen, P.; Geitner, N. K.; Brown, J. M.; Ke, P. C. Nanoscale 2013, 5 (19), 9162-9169.

(226) Karsisiotis, A. I.; Hessari, N. M.; Novellino, E.; Spada, G. P. Angew. Chem. Int. Ed. 2011, 50 (45), 10645-10648.

(227) Mergny, J.-L.; Phan, A.; Lacroix, L. 1998, 435, 74-78.

(228) Mergny, J-L.; Lacroix, L. Curr. Protoc. Nucleic Acid Chem. 2009, 37 (1), 1-15.

(229) Frost, N. R.; McKeague, M.; Falcioni, D.; DeRosa, M. C. Analyst 2015, 140 (19), 6643-6651.

(230) Katayama, E.; Nonomura, Y. J. Biochem. 1979, 86, 1495-1509.

(231) Tzaphlidou, M.; Chapman, J. A.; Al-Samman, M. H. Micron. (1969) 1982, 13 (2), 133-145.

(232) Greenlee, T. K.; Ross, R.; Hartman, J. L. J. Cell Biol. 1966, No. 30, 59-71.

(233) Tran, H. T.; Chung, C. H. Y.; Iba, M.; Zhang, B.; Trojanowski, J. Q.; Luk, K. C.; Lee, V. M. Y. Cell Rep. 2014, 7 (6), 2054-2065.

(234) Games, D.; Valera, E.; Spencer, B.; Rockenstein, E.; Mante, M.; Adame, A.; Patrick, C.; Ubhi, K.; Nuber, S.; Sacayon, P. J. Neurosci. 2014, 34 (28), 94419454.

(235) Näsström, T.; Gonçalves, S.; Sahlin, C.; Nordström, E.; Sundquist, V. S.; Lannfelt, L.; Bergström, J.; Outeiro, T. F.; Ingelsson, M. PLoS One 2011, 6 (10), 1-8.

(236) Näsström, T.; Fagerqvist, T.; Barbu, M.; Karlsson, M.; Nikolajeff, F.; Kasrayan, A.; Ekberg, M.; Lannfelt, L.; Ingelsson, M.; Bergström, J. Free Radic. Biol. Med. 2011, 50 (3), 428-437.

(237) Gai, W. P.; Pountney, D. L.; Power, J. H. T.; Li, Q. X.; Culvenor, J. G.; McLean, C. A.; Jensen, P. H.; Blumbergs, P. C. Exp. Neurol. 2003, 181 (1), 68-78.

(238) Giasson, B. I.; Murray, I. V. J.; Trojanowski, J. Q.; Lee, V. M. Y. J. Biol. Chem. 2001, 276 (4), 2380-2386.

(239) Biere, A. L.; Wood, S. J.; Wypych, J.; Steavenson, S.; Jiang, Y.; Anafi, D.; Jacobsen, F. W.; Jarosinski, M. A.; Wu, G. M.; Louis, J. C. J. Biol. Chem. 2000, 275 (44), 34574-34579.

(240) McClendon, S.; Rospigliosi, C. C.; Eliezer, D. Protein Sci. 2009, 18 (7), 1531-

(241) Rospigliosi, C. C.; McClendon, S.; Schmid, A. W.; Ramlall, T. F.; Barré, P.; Lashuel, H. A.; Eliezer, D. J. Mol. Biol. 2009, 388 (5), 1022-1032.

(242) Takenouchi, T.; Hashimoto, M.; Hsu, L. J.; Mackowski, B.; Rockenstein, E.; 
Mallory, M.; Masliah, E. Mol. Cell. Neurosci. 2001, 17 (1), 141-150.

(243) Paleologou, K. E.; Schmid, A. W.; Rospigliosi, C. C.; Kim, H. Y.; Lamberto, G. R.; Fredenburg, R. A.; Lansbury, P. T.; Fernandez, C. O.; Eliezer, D.; Zweckstetter, M. J. Biol. Chem. 2008, 283 (24), 16895-16905.

(244) Wu, L.; Yao, Y.; Li, Z.; Zhang, X.; Chen, J. J. Electroanal. Chem. 2015, 757, 243249.

(245) Fan, L.; Zhao, G.; Shi, H.; Liu, M.; Li, Z. Biosens. Bioelectron. 2013, 43 (1), 1218.

(246) Abbott, N.J.; Patabendige, A.A.; Dolman, D.E.; Yusof, S.R.; Begley, D. J. Neurobiol. Dis. 2010, 3 (7), 13-25.

(247) Abbott, N.J.; Ronnback, L.; Hansson, E. Nat. Rev. Neurosci. 2006, 7, 41-53.

(248) McConnell, E. M.; Ventura, K.; Dwyer, Z.; Hunt, V.; Koudrina, A.; Holahan, M. R.; Derosa, M. C. ACS Chem. Neurosci. 2019, 10 (1), 371-383.

(249) Pardridge, W. M. Curr. Opin. Pharmacol. 2006, 6 (5), 494-500.

(250) Jefferies, W.A.; Brandon, M.R.; Hunt, S.V.; Williams, A.F.; Gatter, K.C.; Mason, D. Y. Nature. 1984, 312, 162-163.

(251) Chen, C. H. B.; Dellamaggiore, K. R.; Ouellette, C. P.; Sedano, C. D.; Lizadjohry, M.; Chernis, G. A.; Gonzales, M.; Baltasar, F. E.; Fan, A. L.; Myerowitz, R. Proc. Natl. Acad. Sci. U. S. A. 2008, 105 (41), 15908-15913.

(252) Porciani, D.; Signore, G.; Marchetti, L.; Mereghetti, P.; Nifosì, R.; Beltram, F. Mol. Ther. - Nucleic Acids 2014, 3, e144.

(253) MacDonald, J.; Houghton, P.; Xiang, D.; Duan, W.; Shigdar, S. Nucleic Acid Ther. 2016, 26 (6), 348-354.

(254) Macdonald, J.; Henri, J.; Goodman, L.; Xiang, D.; Duan, W.; Shigdar, S. ACS Chem. Neurosci. 2017, 8 (4), 777-784.

(255) Macdonald, J.; Denoyer, D.; Henri, J.; Jamieson, A.; Burvenich, I. J. G.; Pouliot, N.; Shigdar, S. Nucleic Acid Ther. 2020, 30 (2), 117-128.

(256) Bangham, A. D.; Standish, M. M.; Watkins, J. C. J. Mol. Biol. 1965, 13 (1), 238252.

(257) Pattni, B. S.; Chupin, V. V.; Torchilin, V. P. Chem. Rev. 2015, 115 (19), 1093810966.

(258) Gao, W.; Hu, C. M. J.; Fang, R. H.; Zhang, L. J. Mater. Chem. B. 2013, 1 (48), 6569-6585.

(259) Sercombe, L.; Veerati, T.; Moheimani, F.; Wu, S. Y.; Sood, A. K.; Hua, S. Front. Pharmacol. 2015, 6, 1-13.

(260) Gardikis, K.; Tsimplouli, C.; Dimas, K.; Micha-Screttas, M.; Demetzos, C. Int. J. Pharm. 2010, 402 (1-2), 231-237.

(261) Gambling, D.; Hughes, T.; Martin, G.; Horton, W.; Manvelian, G. Anesth. Analg. 2005, 100 (4), 1065-1074.

(262) Stewart, J. C. M. Anal. Biochem. 1980, 104 (1), 10-14.

(263) Miller, C. R.; Bondurant, B.; McLean, S. D.; McGovern, K. A.; O’Brien, D. F. Biochemistry 1998, 37 (37), 12875-12883.

(264) Campbell, R. B.; Fukumura, D.; Brown, E. B.; Mazzola, L. M.; Izumi, Y.; Jain, R. K.; Torchilin, V. P.; Munn, L. L. Cancer Res. 2002, 62 (23), 6831-6836.

(265) Allen, T.M.; Cullis, P. R. Adv. Drug Deliv. Rev. 2013, 65, 36-48.

(266) Nagle, J. F.; Tristram-Nagle, S. Biochim. Biophys. Acta - Rev. Biomembr. 2000, 
1469 (3), 159-195.

(267) Koster, K. L.; Webb, M. S.; Bryant, G.; Lynch, D. V. BBA - Biomembr. 1994, 1193 (1), 143-150.

(268) Kastantin, M.; Ananthanarayanan, B.; Karmali, P.; Ruoslahti, E.; Tirrell, M. Langmuir 2009, 25 (13), 7279-7286.

(269) Szoka, F.; Papahadjopoulos, D. Proc. Natl. Acad. Sci. U. S. A. 1978, 75 (9), 4194. 